University of Louisville

ThinkIR: The University of Louisville's Institutional Repository

Electronic Theses and Dissertations

$12-2015$

\title{
Electrochemical formation of one-dimensional metal nanostructures across microgaps electrodes for resistive switching and optical sensing applications.
}

Nidhi Shah

University of Louisville

Follow this and additional works at: https://ir.library.louisville.edu/etd

Part of the Analytical Chemistry Commons

\section{Recommended Citation}

Shah, Nidhi, "Electrochemical formation of one-dimensional metal nanostructures across microgaps electrodes for resistive switching and optical sensing applications." (2015). Electronic Theses and Dissertations. Paper 2289.

https://doi.org/10.18297/etd/2289

This Doctoral Dissertation is brought to you for free and open access by ThinkIR: The University of Louisville's Institutional Repository. It has been accepted for inclusion in Electronic Theses and Dissertations by an authorized administrator of ThinkIR: The University of Louisville's Institutional Repository. This title appears here courtesy of the author, who has retained all other copyrights. For more information, please contact thinkir@louisville.edu. 
ELECTROCHEMICAL FORMATION OF ONE-DIMENSIONAL METAL NANOSTRUCTURES ACROSS MICROGAP ELECTRODES FOR RESISTIVE SWITCHING AND OPTICAL SENSING APPLICATIONS By

Nidhi Shah

B.S., Maharaja Sayajirao University of Baroda, 2004

M.S., Maharaja Sayajirao University of Baroda, 2006

M.S., University of Louisville, 2013

\begin{abstract}
A Dissertation
Submitted to the Faculty of the College of Arts and Sciences of the University of Louisville in Partial Fulfillment of the Requirements for the Degree of
\end{abstract}

Doctor of Philosophy in Chemistry

Department of Chemistry

University of Louisville

Louisville, Kentucky

December, 2015 
Copyright (C) 2015 by Nidhi Shah

All rights reserved 

ELECTROCHEMICAL FORMATION OF ONE-DIMENSIONAL METAL NANOSTRUCTURES ACROSS MICROGAP ELECTRODES FOR RESISTIVE SWITCHING AND OPTICAL SENSING APPLICATIONS

\author{
By \\ Nidhi Shah \\ A Dissertation Approved on \\ November 18, 2015 \\ by the following Dissertation Committee:
}

Dr. Francis P. Zamborini

(Dissertation Director)

Dr. Muriel C. Maurer

Dr. Richard P. Baldwin

Dr. Gamini U. Sumanasekera 


\section{DEDICATION}

This dissertation is dedicated to my parents

Mr. Jitendra Shah

and

Mrs. Ila Shah

They have always been my guide and source of inspiration. 


\section{ACKNOWLEDGEMENTS}

I am deeply indebted to my research advisor, Dr. Francis P. Zamborini, for his understanding, wisdom, patience, enthusiasm, motivation and encouragement on research. I would like to thank him for being a great and a supportive mentor throughout my Ph.D. studies. Without his guidance, mentoring and knowledge, this study would not have been completed.

I would to like to thank Dr. Richard P. Baldwin, Dr. Muriel C. Maurer and Dr. Gamini U. Sumanasekera, for their time and feedback and for serving on my Ph.D. dissertation defense committee. I also would like to thank all current and former group members. I would like to specially thank Dr. Radhika Dasari for mentoring me since the day of joining the group and for always being willing to listen to my difficulties and give me constructive discussions and give me valuable suggestions. I am thankful to all my friends at University of Louisville especially Dr. Aiqin, Dr. Davinder, Dr. Deepika, and Dr. Manish for their friendship.

I would like to thank Sherry Nalley, Sabrina Haug, and Aaron Howell for their support and help during this tenure. I am very thankful to late Mr Tracy Larson for all his help with stockroom supplies. May his soul rest in peace. I am also very thankful to Renu Kakkar for her help in stockroom chemical supplies and Steve Riley for his help in many technical problems. 
I am thankful to various funding agencies; National Science Foundation and University of Louisville for teaching assistantship. I am also thankful to Graduate Student Council at the University of Louisville for travel funds for conferences. The doctoral dissertation completion award from School of Interdisciplinary and Graduate Studies (SIGS) was a great support during my graduate studies.

Dr. Tereza Paronyan from the ElectroOptics Research Institute and Nanotechnology Center deserves special thanks for her assistance with Scanning Electron Microscopy (SEM), Atomic Force Microscopy (AFM) and Raman studies. I appreciate the help from Dr. Julia Aebersold, Dr Evgenia Moiseeva and Curt McKenna from Micro Nano Technology Center on fabrication of electrode devices.

Last but not the least, I am very thankful to my husband, Nirav Shah who patiently listened and discussed ideas about my project while morally supporting me throughout these years. Without the unconditional love and support from my husband and my daughter Jaini Shah, I would not have been able to complete the studies. Words cannot express how grateful I am to my parents-in-laws Arun Shah and Jyotsna Shah and my parents Jitendra Shah and Ila Shah for all of the sacrifices that you've made on my behalf. Your prayer for me was what sustained me thus far. I would like to thank my sister in-law Dr. Hiral Shah and my brother Dhruvin Shah for their moral support and constant encouragement. I would also like to thank CBS family and Taramandal family and all my uncles, aunts and cousins for their support and love. 


\begin{abstract}
ELECTROCHEMICAL FORMATION OF ONE-DIMENSIONAL METAL NANOSTRUCTURES ACROSS MICROGAP ELECTRODES FOR RESISTIVE SWITCHING AND OPTICAL SENSING APPLICATIONS
\end{abstract}

Nidhi Shah

November 18,2015

This dissertation focuses on the electrochemical formation of onedimensional (1D) Ag nanostructures (in particular nanowires and nanochains) directly on electrode surfaces or gap of two electrodes. Ag nanowires (NWs) and nanorods (NRs) grow directly on glass/indium tin oxide (ITO) surfaces from electrode-attached Au nanoparticle seeds in the presence of cetyltrimethylammonium bromide $(\mathrm{CTAB})$ at appropriate potentials with a $5-15 \%$ yield and the average length ranging from $100 \mathrm{~nm}$ to $3 \mu \mathrm{m}$ for growth times of 30 to $120 \mathrm{~min}$. This method failed when trying to grow Ag NRs/NWs across $5 \mu \mathrm{m}$ Au electrode gaps. Interestingly, applying voltage in air leads to the formation of 1D Ag nanoparticle (Ag NP) chains across the electrode gap in the presence of CTAB and humidity. This is due to an electrochemical process of Ag oxidation at the positive electrode and reduction of $\mathrm{Ag}^{+}$at the negative electrode of the gap. The 1D Ag NP chains have interesting resistive switching properties with ON/OFF ratio $>100$, endurance cycles of at least 1000 and ms switching speeds, which could find use for memory devices. A device formed by electrodeposition of polyphenol on one electrode and Ag NWs on a second electrode of a $5 \mu \mathrm{m}$ electrode gap also exhibits resistive switching properties similar to 1D Ag NP 
chains. Interestingly, the Ag NWs convert into Ag NP chains during the first connection. 1D Ag NP chains formed electrochemically in the presence of CTAB show interesting surface-enhanced Raman and localized surface plasmon resonance spectroscopy that depends on their conductance state. 


\section{TABLE OF CONTENTS}

CHAPTER

PAGE



ACKNOWLEDGEMENTS.............................................................. iv

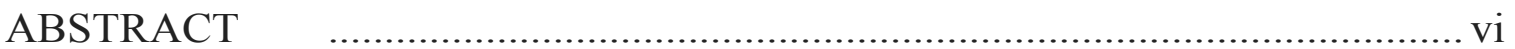

LIST OF FIGURES ....................................................................





1.1 Main Goal And Summary ............................................................... 1

1.2 Motivation /Objective ............................................................. 2

1.3 1D Metal Nanostructures And Their Importance....................................... 4

1.4 Synthesis Of 1D Metal Nanostructures .............................................. 4

1.4.1 Template Directed Synthesis ..................................................... 5





1.4.4 Electrochemical Synthesis ............................................................ 9

1.5 Assembly Of 1D Metal Nanostructures ............................................... 10

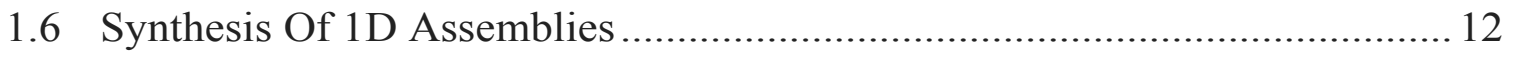



1.6.2 Assembly By External Forces ................................................... 14

1.6.3 Assembly By Biomolecule Linkers ............................................. 15

1.6.4 Assembly By Interparticle Forces ........................................... 16

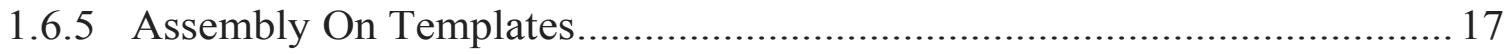






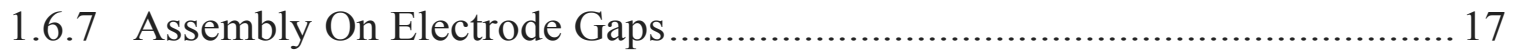

1.7 Two Terminal Resistive Switching In 1D Metal Nanostructures .................. 18

1.8 Simultaneous Measurements Of Electronic Conduction And Raman Response

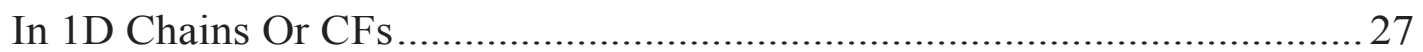



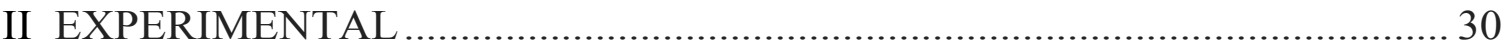

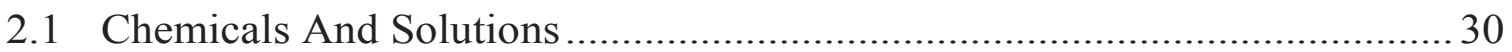



2.1.2 Synthesis Of 3-5 Nm Diameter Au Nanosphere (NS) Seeds ...................... 30





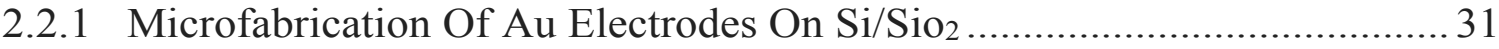

2.2.2 Microfabrication Of Au Electrodes On Glass ............................................ 31

2.2.3 Wiring And Cleaning Of The Au IDA Or TED Electrodes .........................33











2.3.2 Scanning Electron Microscopy (SEM) ………................................... 42

2.3.3 Surface Enhanced Raman Spectroscopy (SERS) And Microscopy ........... 42

2.3.4 Simultaneous Conductivity And SERS Mapping Measurements ............... 43

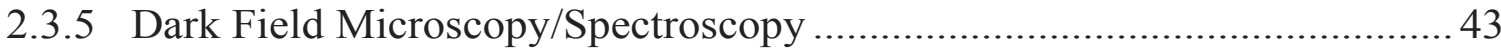


2.4 Chemical And Electrochemical Procedures.

2.4.1 Electrodeposition Of Ag On 5 Micron Gap Au Electrodes Using Cyclic



2.4.2 Electrodeposition Of Ag on 5 micron gap Au Electrodes using









2.4.6 Electrodeposition Of Ag Nws On Au IDA...........................................52

2.4.7 Ag Electrodeposition On Glass/ITO ....................................................... 54





III ELECTROCHEMICAL DEPOSITION OF AG NANOWIRES/NANORODS BY SEED-MEDIATED GROWTH DIRECTLY ONTO ELECTRODE

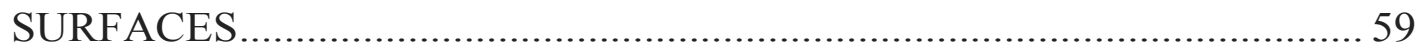





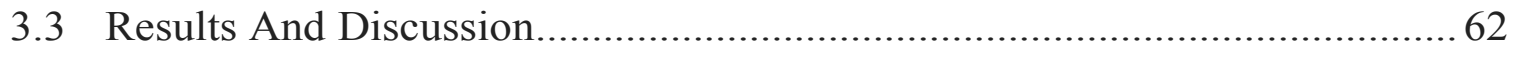

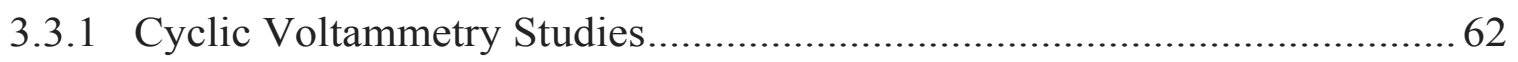

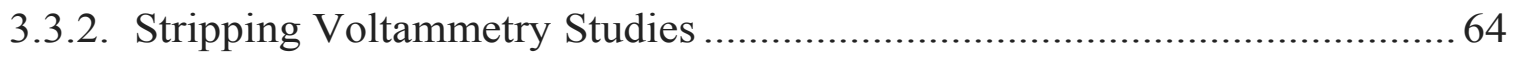

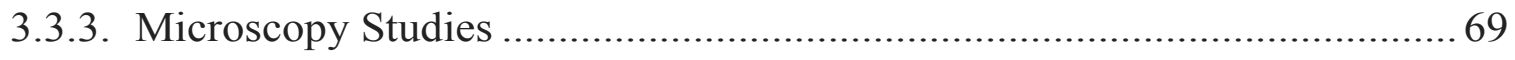

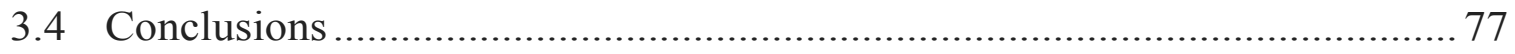


IV RESISTIVE SWITCHING IN METAL/AG NW/POLYPHENOL/METAL









4.3.1 Fabrication Of Metal/Polyphenol/Ag NW/Metal Device........................... 82

4.3.2 Resistive Switching In The Presence Of Humid Air .................................. 85

4.3.3 Resistive Switching In The Presence Of Nitrogen ...................................... 91

4.3.4 Possible Mechanism Of Ag Nano Particle Chain Formation And Resistive





V SURFACTANT-ASSISTED VOLTAGE-DRIVEN SILVER

NANOPARTICLE CHAIN FORMATION ACROSS MICROELECTRODE









5.3.1 Electrodeposition Of Ag On Au IDA Devices ........................................ 106

5.3.2 Formation Of One-Dimensional Ag Nanoparticle Chain Formation Across

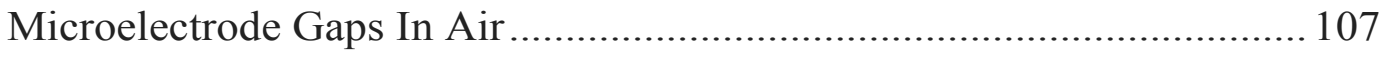

5.3.3 Real-Time Dark Field Microscopy Snap Shots Of Au Two Electrode

Device 114

5.3.4 Control Experiments Critical To Ag NP Chain Formation 120 
5.3.5 Role Of Humidity On Ag NP Chain Formation 123

5.3.6 Determination Of Mechanism For The Formation Of Ag NP Chains ...... 127

5.3.7 Explanation Of Scheme Of Electrochemical Mechanism For Ag NP Chain

Formation 135

5.4 Conclusions

VI RESISTIVE SWITCHING BEHAVIOR OF ELECTROCHEMICALLY SYNTHESIZED ONE-DIMENSIONAL SILVER NANOPARTICLE CHAINS 140



6.2 Experimental Section ............................................................ 144

6.3 Results And Discussion...................................................................... 145

6.3.1 Resistive Switching Of IDA Electrodes With Ag Electrodeposited On Both Sides Of The Device. 145

6.3.2 Resistive Switching Of Ag Nanoparticle Chains Formed Across The $5 \mu \mathrm{m}$ Gap Of TED 151

6.3.3 Mechanism Of Resistive Switching Of Ag Nanoparticle Chains.... 161

6.4 Conclusions 171

VII OPTICAL CHARACTERIZATION OF 1D AG NANOCHAINS USING SURFACE ENHANCED RAMAN SPECTROSCOPY AND RAYLEIGH DARK-FIELD SCATTERING SPECTROSCOPY

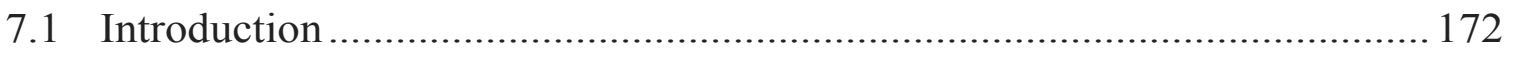

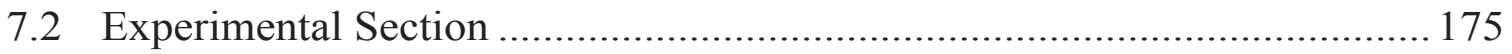

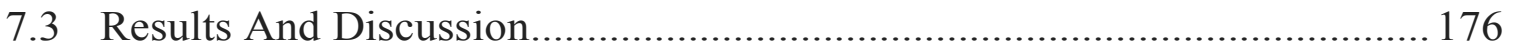




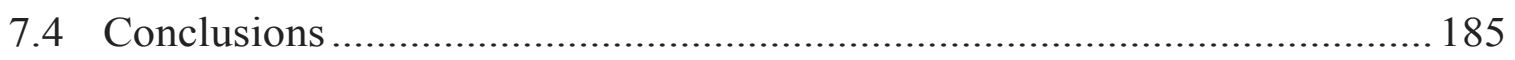

VIII SUMMARY AND FUTURE DIRECTIONS ..................................... 189

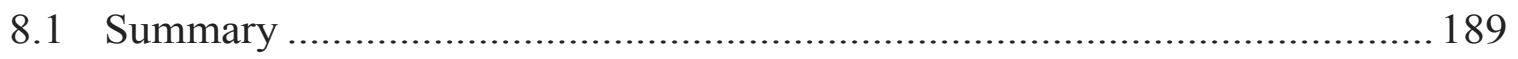

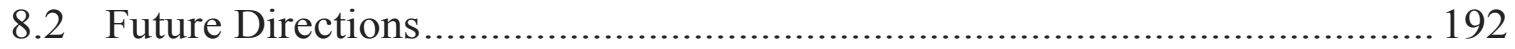

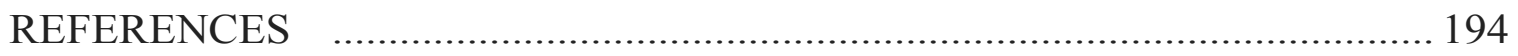



CURRICULUM VITAE ..................................................................... 208 


\section{LIST OF FIGURES}

FIGURE

PAGE

1.1. Illustration of the seed-mediated growth method 8

1.2. Scheme showing arrangement of metal nanoparticles into $1 \mathrm{D}, 2 \mathrm{D}$ and $3 \mathrm{D}$ assemblies.

1.3. CFs formed by (A) anionic migration involving migration of oxygen vacancies and (B) cationic migration involving migration of $\mathrm{Ag}$ ions.

2.1. Optical images of bare Au $5 \mu \mathrm{m}$ gap (A) Au IDA device on $\mathrm{Si} / \mathrm{SiO}_{2}$ and (B) Au TED on $\mathrm{Si} / \mathrm{SiO}_{2}$.

2.2. Dark-field microscopy images of Au TED with gap size of (A) $5 \mu \mathrm{m} \mathrm{Au}$ TED, (B) $10 \mu \mathrm{m}$ Au TED and (C) $23 \mu \mathrm{m}$ Au TED.

2.3. Steps involved in wiring and cleaning the electrode devices.

2.4. Cyclic voltmmogram of Au IDA in $0.1 \mathrm{M} \mathrm{H}_{2} \mathrm{SO}_{4}$ shows oxidation and reduction peaks of Au. 36

2.5. Scheme showing steps involved in fabricating glass/ITO/MPTMS/Au NP (4 $\mathrm{nm})$ electrode.

2.6. Digital photograph showing conventional three electrode set up for metal electrodeposition

2.7. Digital photograph showing the two electrode setup for I-V or I-t measurements in air in CV or CA mode, respectively, of the CHI660A 41 
2.8. Instrumental set up for simultaneou Raman and electrical conductance measurement of Ag NP chain coated with 4-ATP on Au TED.

2.9. A) Apparatus for measuring dark-field microscopy and spectroscopy. B) Diagram showing the conical illumination. Yellow lines represent transmitted light missing the objective and green lines represent the scattered light detected by the objective.

2.10. Linear Sweep Voltammogram with the reference electrode and counter electrode only in $2.5 \times 10^{-4} \mathrm{M} \mathrm{AgNO}_{3}$ in $0.1 \mathrm{M} \mathrm{CTAB}$ phosphate buffer solution. After pausing at potential at $-0.2 \mathrm{~V}$, the appropriate IDA device or TED was immersed in the solution and held for a desired amount of time at this potential for Ag deposition. It was also removed while still under potential control.

2.11. Chronocoulometric plot of IDA or TED electrode in $10 \mathrm{mM} \mathrm{AgNO3} \mathrm{in}$ water at $-0.3 \mathrm{~V}$

2.12. SEM images of IDA device with Ag deposited on each side. (A) $\mathrm{Au} /$ dithiol/Au NPs/Ag device with Ag deposited using cyclic voltammetry in the presence of CTAB and (B) Au/dithiol/Au NPs/Ag device with Ag deposited using chronocoulometry in the absence of CTAB. (C) and (D) are zoom in images of circled part shown in (A) and (B)

2.13. Cyclic voltammetry of one side of an Au IDA device in $5 \mathrm{mM}$ phenol in 0.1 M H2SO4 for the first 10 cycles. Phenol oxidation at $1.1 \mathrm{~V}$ on the first cycle leads to a polyphenol film on the electrode that blocks phenol oxidation in 
subsequent scans. Electropolymerization of phenol on one set of Au IDA electrodes. I-V plot shows first 10 cycles ................................................... 53

2.14. Ag deposition on Au IDA using chronocoulometry technique .................. 55

2.15. Set up for the Electrochemical Seed-Mediated Growth of Ag ....................56

2.16 A schematic illustration of the growth of Ag nanostructures directly on the surfaces of glass/ITO by the seed-mediated growth procedure.

3.1. CVs of glass/ITO (blue), glass/ITO/MPTMS (red) and glass/ITO/MPTMS/Au NP seed (black) in $0.1 \mathrm{M} \mathrm{CTAB}$ plus $2.5 \times 10^{-4} \mathrm{M}$ $\mathrm{AgNO}_{3}$ in $\mathrm{pH} 10.6$ buffer from $0.2 \mathrm{~V}$ to $-1.2 \mathrm{~V}$.

3.2. (A) Stripping Voltammogram for glass/ITO/MPTMS and glass/ITO/MPTMS/Au NP seeds after Ag deposition at $-0.2 \mathrm{~V}$ for $30 \mathrm{~min}$. (B) Stripping voltammetry charge of glass/ITO/MPTMS (red) and glass/ITO/MPTMS/Au NP seed (black) electrodes after Ag deposition at different potentials for $30 \mathrm{~min}$ in $\mathrm{pH} 10.60 .1 \mathrm{M} \mathrm{CTAB}$ and $2.5 \times 10^{-4} \mathrm{M}$ $\mathrm{AgNO}_{3}$.

3.3. Stripping voltammetry of glass/ITO/MPTMS (red) and glass/ITO/MPTMS/Au NP seed (black) electrodes after Ag deposition at the different indicated potentials for $60 \mathrm{~min}$ in $0.1 \mathrm{M} \mathrm{CTAB}$ and $2.5 \times 10^{-4} \mathrm{M}$ $\mathrm{AgNO}_{3}$ at $\mathrm{pH} 10.6$

3.4. Stripping Voltammetry data of glass/ITO/MPTMS (red) and glass/ITO/MPTMS/Au NP seed (black) electrodes held at different potentials for $120 \mathrm{~min}$ in $0.1 \mathrm{M} \mathrm{CTAB}$ and $2.5 \times 10^{-4} \mathrm{M} \mathrm{AgNO}_{3}$ 68 
3.5. Stripping charge vs Ag deposition time plot for glass/ITO/MPTMS (red) and glass/ITO/MPTMS/Au NP seed (black) for potentials $-150,-185$ and $\quad-200$

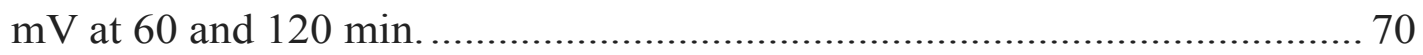

3.6. SEM images of glass/ITO/MPTMS at the indicated potentials for a deposition time of 30min.

3.7. SEM images of glass/ITO/MPTMS (A, B, C) and glass/ITO/MPTMS/Au NP seed electrodes (D, E, F) held at $-150 \mathrm{mV}$ for 30,60 and $120 \mathrm{~min}$ 72

3.8. SEM images of glass/ITO/MPTMS (A, B, C) and glass/ITO/MPTMS/Au NP seed electrodes (D, E, F) held at $-185 \mathrm{mV}$ for 30,60 and $120 \mathrm{~min}$ 74

3.9. SEM images of glass/ITO/MPTMS (A, B, C) and glass/ITO/MPTMS/Au NP seed electrodes (D, E, F) held at $-200 \mathrm{mV}$ for 30, 60 and $120 \mathrm{~min}$ 75

Scheme 4.1. Schematic illustrating the procedure for the fabrication of electrode(E1)/Ag NW/polyphenol/electrode (E2) devices. 83

4.1. I-V plot of 1) Au E1/Air/Au E2 (Black), 2) Au E1/Air/polyphenol/Au E2 (Blue) after polyphenol deposition for 100 cycles at E2, and, 3) Au E1/AgNW/polyphenol/Au E2 (Red) after Ag deposition at E1 84

4.2. SEM images of the Au IDA device after different steps for the fabrication of electrode/polyphenol/Ag NW/electrode device. (A) E1/Air/E2 IDA. (B) E1/Air/polypheno1/E1 IDA and (C) E1/Ag NW/polyphenol/E2 IDA. 86

4.3. I-V plots of the electrode/Ag NW/polyphenol/electrode device scanned from $0 \mathrm{~V}$ to $-5 \mathrm{~V}$ and $-5 \mathrm{~V}$ to $0 \mathrm{~V}$. Black arrows represent forward scan and red arrows represent reverse scan. (A) Scan 1 shows a connection formed on the reverse scan. (B) Scan 2 shows good electrical behavior for resistive 
switching with high resistance negative of $-1.0 \mathrm{~V}$ on the forward scan and low resistance positive of $-2.5 \mathrm{~V}$ on the reverse scan.

4.4. Current-time plot of the device after performing the I-V curves as shown in Figure 4.3.

4.5. SEM images of the device after the performing I-V studies and switching cycles.

4.6. Current-time plot of an Au E1/Ag NW/Polyphenol/Au E2 device showing a current increase at the indicated voltage due to AgNP arrays spanning the electrode gap.

4.7. I-V plot after I-t plot shown in Figure 4.6

4.8. Current time plot for two different programming speeds. (A) $0.25 \mathrm{~s}$ (B) $0.025 \mathrm{~s}$.

4.9. Endurance of the device after performing I-t plots shown in Figure 4.8. The device showed endurance for at least 1000 cycles. (A) first 20 cycles of 1000 cycles and (B) last 20 cycles of 1000 cycles. There is almost no change in the $\mathrm{ON}$ and OFF currents. 96

4.10. Current-time plot showing the ON (A) and OFF (B) current levels for the device. The ON/OFF current ratio is $\sim 1000$.

4.11. SEM images of the device after performing all switching experiments. SEM and optical images are consistent with each other, showing the Ag NP chains forming from AgNW at the locations marked by numbers 1, 2 and 3.98

5.1. I-V curve of an untreated bare Au IDA device (grey) and Au/dithiol/Au NPs IDA device after Ag electrodeposition (red). The fact that the current is the 
same as the bare Au IDA device after Ag electrodeposition shows that the Ag did not make a connection across the 5 micron IDA gap. 108

5.2. SEM images of $(A, C)$ Bare Au IDA device and $(B, D) A u /$ dithiol/Au NPs IDA device after Ag electrodeposition on each side. There is no obvious connection between the two electrodes of the IDA after Ag deposition, which explains the pA level current in the I-V curve of Figure 5.1. The circled regions in $\mathrm{A}$ and $\mathrm{B}$ are the regions shown in the magnified images of $\mathrm{C}$ and

D. 109

5.3. Current-time plots obtained at the indicated voltages on IDA devices with Ag deposited in the presence of CTAB on both sides of (A) bare Au, (B) $\mathrm{Au} / \mathrm{dithiol}$, and (C) Au/dithiol/Au NPs devices. 110

5.4. SEM images of various treated IDAs after Ag deposition in the presence of $\mathrm{CTAB}$ and application of an applied voltage for various times. (A,D) bare $\mathrm{Au} / \mathrm{Ag}$ IDA after applying $5 \mathrm{~V}$ and $10 \mathrm{~V}$ as shown in Figure 5.3A. (B,E) $\mathrm{Au} /$ dithiol/Ag IDA after applying $5 \mathrm{~V}$ and $10 \mathrm{~V}$ as shown in Figure 5.3B. (C,F) $\mathrm{Au} /$ dithiol/Au NPs/Ag IDA after applying $5 \mathrm{~V}$ as shown in Figure 5.3C. Chains of Ag NPs formed across the electrode gap, explaining the large 8-9 order of magnitude increase in current in the I-t plots in Figure 5.3. The circled areas are the regions that are expanded in the adjacent SEM images to the right

5.5. Ag deposition with $\mathrm{CTAB}$ on one side of the device. (A) Bare $\mathrm{Au} / \mathrm{Ag}$, (B) $\mathrm{Au} / \mathrm{dithiol} / \mathrm{Ag}$, and (C) Au/dithiol/Au NPs/Ag. The electrode with dithiol, 
Au seeds, and Ag showed spikes corresponding to Ag NP chain formation after applying $10 \mathrm{~V}$ with the $\mathrm{Ag}$ side positive

5.6. (A) SEM image of an IDA device with Au/dithiol/Au NPs/Ag on one side after application of $10 \mathrm{~V}$ (Ag side positive) as shown in Figure 5.5C. (B) Zoomed-in image of circled part in (A).

5.7. Current-time plot at $10 \mathrm{~V}$ (top) and corresponding dark-field microscopy images at (A) $120 \mathrm{~s}$, (B) $128 \mathrm{~s}$, (C) $150 \mathrm{~s}$, and (D) $158 \mathrm{~s}$ of a two electrode device on glass with bare $\mathrm{Au}$ on the negative electrode and $\mathrm{Au} /$ dithiol/Au $\mathrm{NPs} / \mathrm{Ag}$ on the positive electrode as indicated in (A). The current increases well before the Ag NP chain can be visualized by dark-field microscopy due to the limited optical resolution. The Ag NP chains start off small and grow with time for 25-30 s until they are visible by dark-field microscopy. 116

5.8. Real-time dark-field microscopy snap shots of an Au two electrode device with $\mathrm{Au} /$ dithiol/Au NPs/Ag on one side held at $10 \mathrm{~V}$ for various amounts of time. The times indicated correspond to the current-time plot shown in Figure 5.9. An initial jump in current occurred about $25 \mathrm{~s}$ before the appearance of Ag NP chain 1. A jump in current appeared during the appearance of chain 1 and chain 2. Chain formation occurs by movement of $\mathrm{Ag}$ from the positive electrode to the negative electrode as indicated by the loss of $\mathrm{Ag}$ on the positive side. 118

5.9. Current-time plot corresponding to the dark-field images shown in Figure 5.8. A small increase in current occurs near $60 \mathrm{~s}$ and a larger one near $95 \mathrm{~s}$, which is about $25 \mathrm{~s}$ before the appearance of the first Ag NP chain, which 
shows another jump in current. The appearance of a second chain occurred with another, but smaller increase in current. The current is dynamic and spiky.

5.10. (A) Current-time plot for two Au/dithiol/Au NPs/Ag devices at $0.5 \mathrm{~V}$ showing Ag NP chain formation at this low voltage between 100s - 400s. (B) SEM image of the device whose current increased after $100 \mathrm{~s}$ in (A). (C) a zoomed-in image of the circled part of (B) showing Ag NP chain formation across a much smaller distance, which explains the low voltage required for Ag NP chain formation in this case.

5.11. Current time plots at $5 \mathrm{~V}$ and $10 \mathrm{~V}$ as indicated from $0 \mathrm{~s}$ to $1000 \mathrm{~s}$ for the device after Ag deposition on (A) bare Au, (B) Au/dithiol, (C) Au/dithiol/Au NPs on both sides and (D) Au/dithiol/Au NPs on one side in the absence of CTAB. No current jump occurred when deposition occurred in the absence of $\mathrm{CTAB}$, indicating no Ag NP chain formation.

5.12. Current-time plots of an electrode device with Au/dithiol/Au NPs/Ag on one side of the device prepared by Ag electrodeposition in the presence of CTAB. Ozone treatment or soaking in water for $2 \mathrm{~h}$ prior to the application of $10 \mathrm{~V}$ (Ag side positive) prevents Ag NP chain formation from occurring. This is likely due to the removal of adsorbed CTAB from the surface, which is necessary for Ag NP chain formation.

5.13. Current-time plots obtained at the indicated voltages on IDA device with $\mathrm{Ag}$ deposited in the presence of CTAB on one side of a Au/dithiol/Au NPs device. The current remained at the instrument background level up to $10 \mathrm{~V}$ 
(Ag side positive) when in the presence of dry $\mathrm{N}_{2}$. The current increased to the mA level due to Ag NP chain formation once the same device was later introduced to humid air. This shows that humidity is crucial for Ag NP chain formation. 125

5.14. I-t plots obtained at the indicated voltages on IDA or TED devices with Ag deposited in the presence of CTAB on one side of the device with Au NP and thiol for different humidity level. (A) $20 \%$, current remained at pA level upto $10 \mathrm{~V}$ for $1000 \mathrm{~s}$, (B) $25 \%$, rise in current was observed at $10 \mathrm{~V}, \sim 40 \mathrm{~s},(\mathrm{C})$ $30 \%$, rise in current was observed at $10 \mathrm{~V}, \sim 210 \mathrm{~s}$, (D) $35 \%$, rise in current observed at $6 \mathrm{~V}, \sim 20 \mathrm{~s},(\mathrm{E}) 40 \%$, rise in current observed at $5 \mathrm{~V}, \sim 70 \mathrm{~s}$ and (F) $50 \%$, rise in current observed at $5 \mathrm{~V}, \sim 2 \mathrm{~s}$. The rise in current for the devices indicated Ag NP chain formation. 126

5.15. SEM images of IDA or TED devices after Ag deposition in presence of $\mathrm{CTAB}$ and application of voltage for various times and formation of chains at different voltages depending upon the percentage level of humidity. (A, G) $20 \%$ humidity level does not show any chains, which is consistent with the current-time plot shown in Figure 5.14A. (B-E) Ag NP chains are formed on IDA or TED devices. We observed that as the humidity level increases, voltage at which chains form decreases. (H-L) Zoomed in images of the circled parts in (B-E)

5.16. Current-time plots at 5 or $10 \mathrm{~V}$ as indicated of IDA devices coated with Ag on both sides and (A) not treated further, (B) soaked in aqueous 0.1 M CTAB for 1 hour, rinsed with water, and dried in $\mathrm{N}_{2}$, and (C) soaked in aqueous $1 \mathrm{x}$ 
$10^{-4} \mathrm{M}$ CTAB solution for 1 hour, not rinsed, and dried in air for 2 hours. This shows that Ag NP chains can form from devices post-treated with $\mathrm{CTAB}$, not just those with $\mathrm{Ag}$ deposited in the presence of CTAB. It also shows that the voltage for Ag NP chain formation decreases as the amount of $\mathrm{CTAB}$ increases. This likely explains why 2 -sided devices required lower voltage than one-sided devices

5.17. SEM images of $\mathrm{Au} / \mathrm{Ag}$ devices $(\mathrm{A}, \mathrm{D})$ untreated, $(\mathrm{B}, \mathrm{E})$ treated with $0.1 \mathrm{M}$ $\mathrm{CTAB}$ and rinsed, and $(\mathrm{C}, \mathrm{F})$ treated with $1 \times 10^{-4} \mathrm{M}$ CTAB but not rinsed, corresponding to the I-t plots A, B, and C in Figure 5.16, respectively. Post treatment with CTAB leads to Ag chain-like structures caused by $\mathrm{Ag}$ movement from the positive to negative electrode, similar to devices with Ag deposited in the presence of CTAB.

5.18 (A) Current-time plot of an $\mathrm{Au} / \mathrm{Ag}$ (two sides) device soaked in 1 x 10-4 M SDS and air dried for 2 hours. (B, C) SEM images of the device after the current-time plot in (A). (D) Current-time plot of an $\mathrm{Au} / \mathrm{Ag}$ device soaked in aqueous $0.1 \mathrm{M}$ SDS for 1 hour, rinsed with water and dried in $\mathrm{N}_{2}$. (E, F) SEM images of the device after the current-time plot in (D). The current increased at $3 \mathrm{~V}$ after $\sim 85 \mathrm{~s}$ or $4 \mathrm{~V}$ after $45 \mathrm{~s}$ due to $\mathrm{Ag}$ chain-like structures growing across the gap. The Ag moved from the positive to negative electrode in the same way as when positively-charged CTAB was used. This suggests an electrochemical mechanism is responsible for the Ag NP chain formation. 
5.19. (A) Current-time plot of an $\mathrm{Au} / \mathrm{Ag}$ device (Ag on one side) soaked in $1 \times 10$ $4 \mathrm{M}$ citrate solution and air dried for 2 hours. $(\mathrm{B}, \mathrm{C})$ SEM images of the device after the current-time plot in (A). The current increased at $3 \mathrm{~V}$ after 5 $\mathrm{s}$ due to $\mathrm{Ag}$ migration from the positive to negative electrode. The Ag got removed from some parts of the electrode and crossed the other side.

5.20. $\mathrm{I}-\mathrm{V}$ plots of $\mathrm{Au} / \mathrm{Ag}$ devices after it was soaked in aqueous $1 \times 10^{-4} \mathrm{M}$ electrolyte solution of (A) CTAB, (B) SDS, and (C) Citrate for 1 hour and allowed to air dry without rinsing for 2 hours

Scheme 5.1. Illustration of electrochemical Ag NP chain formation mechanism between the two Au electrodes.

6.1 Current-time plot obtained in air at the indicated voltages on a $5 \mu \mathrm{m}$ gap Au IDA device functionalized with octanedithiol and $4 \mathrm{~nm}$ Au NPs before electrodeposition of $\mathrm{Ag}$ in the presence of $\mathrm{CTAB}$ on both sides. The increase in current with fluctuations is a characteristic of the Ag NP chain formation process across the electrode gap. 146

6.2. I-V characteristics of an IDA device with Ag deposition in presence of $\mathrm{CTAB}$ on both sides after applying a voltage of $5 \mathrm{~V}$ from 0 to $1000 \mathrm{~s}$ on a $\mathrm{Au} /$ dithiol/Au NPs device. The device was scanned from $0 \mathrm{~V}$ to $-5 \mathrm{~V},-5 \mathrm{~V}$ to $0 \mathrm{~V}, 0$ to $5 \mathrm{~V}$ and $5 \mathrm{~V}$ to $0 \mathrm{~V}$. (A) The device is at LRS initially and goes to HRS from $-4 \mathrm{~V}$ to $-4.8 \mathrm{~V}$ on forward scan and later back to LRS from $-5 \mathrm{~V}$ to $0 \mathrm{~V}$ on reverse scan. (B) The device shows distinct LRS and HRS with LRS from 0 to $-4.8 \mathrm{~V}$ on forward scan and HRS from $-5 \mathrm{~V}$ to $-4.5 \mathrm{~V}$ and again LRS from $-4.5 \mathrm{~V}$ to $0 \mathrm{~V}$ on reverse scan. (C) The device showing LRS and 
HRS with LRS from 0 to $4.8 \mathrm{~V}$ on forward scan and HRS from $5 \mathrm{~V}$ to $2.2 \mathrm{~V}$ and again LRS from $2.2 \mathrm{~V}$ to $0 \mathrm{~V}$ on reverse scan. Black arrows indicate forward scan and red arrows indicate reverse scan.

6.3. Au/Ag NP chain/Au device tested for 20 cycles with switching times (A) $0.025 \mathrm{~s}$ and $(\mathrm{B}) 0.0025 \mathrm{~s}$ showing $\mathrm{ON} / \mathrm{OFF}$ ratio $\sim 10^{2}$ where $\mathrm{HRS}=3-4 \times 10^{-}$

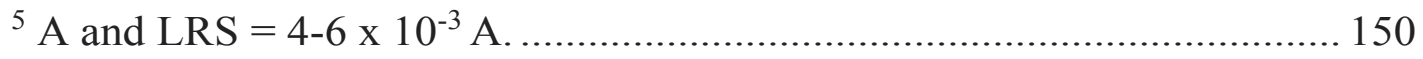

6.4. Current levels of the device after performing switching speed studies (A) ON state and (B) OFF state. 152

6.5. Endurance studies for the $\mathrm{Au} / \mathrm{Ag} \mathrm{NP}$ chain/Au device showing the (A) first 20 cycles and (B) last 20 cycles of 1000 cycles total where HRS $=3-5 \times 10^{-4}$ A and $\mathrm{LRS}=1-6 \times 10^{-3} \mathrm{~A}$. 153

6.6. Current levels of the device after performing enudurance studies (A) ON state and (B) OFF state.

6.7. SEM images of the device before and after performing chain formationand switching operations. (A) SEM image of the bare IDA device, (C) SEM image of $\mathrm{Au} / \mathrm{dithiol} / \mathrm{Au}$ NPs after electrodeposition of Ag on both sides in the presence of CTAB and (E) SEM image of Au/Ag NP/Au IDA device after chain formation and the switching operations. (B, D, F) show zoomed in regions of the circles shown in $(\mathrm{A}, \mathrm{C}, \mathrm{E})$.

6.8. Current-time plot obtained at the indicated the voltage on an IDA device with Ag deposited in the presence of CTAB on one side of $\mathrm{Au} / \mathrm{dithiol} / \mathrm{Au}$ NPs devices. 
6.9. I-V scans after the formation of Ag NP chain where Ag side is positive and Au side is negative. (A) Device is at LRS from 0 to $-2.5 \mathrm{~V}$ and HRS from $2.5 \mathrm{~V}$ to $-5 \mathrm{~V}$ in the forward scan. Device shows HRS from $-5 \mathrm{~V}$ to $-3.5 \mathrm{~V}$ and remains LRS throughout the completion of the cycle on reverse scan. (B) Device at LRS state from 0 to $-3.2 \mathrm{~V}$ and HRS from $-3.2 \mathrm{~V}$ to $-5 \mathrm{~V}$ in the forward scan. In the reverse scan, device is at HRS from $-5 \mathrm{~V}$ to $-3 \mathrm{~V}$ and remain in LRS throughout the cycle. In both cases, the device shows unipolar behavior where HRS and LRS can be seen at the same polarity. The lack of hysteresis in the forward and reverse scan makes it difficult to identify a good read voltage.

6.10. Current-time plot of Au/Ag NP chain/Au TED performed after I-V scans shown in Figure 6.9. The device was switched from $-0.5 \mathrm{~V}$ to $-5 \mathrm{~V}$ for 20 cycles with a pulse width of $1 \mathrm{~s}$. The device is ohmic for most switches and not showing resistive switching behavior. It is in LRS at both $-0.5 \mathrm{~V}$ and -5 V.

6.11. I-V plot after performing I-t plot as shown in Figure 6.10. Device showed unipolar switching with distinct $\mathrm{ON}$ and OFF state and nice hysteresis necessary for identifying a read voltage. Ag side was kept positive and $\mathrm{Au}$ side was kept negative during switching. 160

6.12. Au/Ag NP chain/Au device tested for 20 cycles with switching times of (A) $0.025 \mathrm{~s}$ and, (B) $0.25 \mathrm{~s}$. The ON/OFF ratio $\sim 10^{2}, \mathrm{HRS}=2-4 \times 10^{-5} \mathrm{~A}$, and LRS $=1-3 \times 10^{-3} \mathrm{~A}$ 162 
6.13. Endurance studies for the Au/Ag NP chain/Au device with $\mathrm{Ag}$ on one side with switching time of $0.5 \mathrm{~s}$ (A) First 20 cycles of 1000 cycles and (B) last 20 cycles of 1000 cycles. HRS $=3-5 \times 10^{-5} \mathrm{~A}$ and LRS $=0.8-3 \times 10^{-3} \mathrm{~A} \ldots \ldots .163$

6.14. Current levels of the device after performing switching operations (A) ON state and (B) OFF state. ON to OFF ratio is $10^{2}$. 164

6.15. SEM images of the TED device before and after chain formation and switching operations (A) Bare TED, (B) Au/dithiol/Au NPs TED with Ag electrodeposited on one side of the device having Au NPs and dithiol, and (C) $\mathrm{Au} / \mathrm{Ag} \mathrm{NP}$ chain/Au TED after performing switching operations. 165

6.16. Au/dithiol/Au NP IDA device with Ag deposited on one side of the device showing the formation of chains at $10 \mathrm{~V}$.

6.17. I-V plots of the device after Ag NP chain formation as shown in Figure 6.16. (A) Device shows LRS from 0 to $-4 \mathrm{~V}$ with current showing spikes and HRS from $-4 \mathrm{~V}$ to $-5 \mathrm{~V}$ in forward scan. Current decays with spikes but does not turn OFF completely. During reverse scan, device shows HRS from $-5 \mathrm{~V}$ to $-3.5 \mathrm{~V}$ and remains LRS thereafter throughout the completion of the cycle. (B) Positive scan showing good switching behavior with hysteresis 168

6.18. Au/dithiol/Au NPs IDA device with Ag on one side of the device. (A) Switching in dry $\mathrm{N}_{2}$ from $0.5 \mathrm{~V}$ to $5 \mathrm{~V}$ for 20 cycles at $0.5 \mathrm{~s}$ pulse width, (B) Switching in the absence of CTAB in air by soaking the device in water for two hours. Device switched from $0.5 \mathrm{~V}$ to $5 \mathrm{~V}$ for 20 cycles at $0.5 \mathrm{~s}$ pulse width. 169 
Scheme 6.1. Resistive switching mechanism showing the ON and OFF states for the $\mathrm{Au} / \mathrm{Ag}$ NP chain/Au devices. Mechanism involves atomic dissolution and migration to form connetions and breaks at specific voltages.

7.1. I-t plot showing chain formation at the indicated voltage.

7.2. (A) Optical image of the device after Ag NP chain formation and (B) SERS spectra of DMAB molecule at the chain region without any application of potential.

7.3. (A) SERS mapping at the chain region showing the intensity map and (B) I-t plot of the device for pulse width $30 \mathrm{~s}$ at $-0.5 \mathrm{~V}$ and $-5 \mathrm{~V}$ carried out simultaneously with Raman mapping. 180

7.4. (A) Current-time plot and Raman map of the device at the Ag NP chain region. The rectangular region in the Raman map labelled as a, b, c, d, and e further correlates to the on and off state in the current-time plot and (B) SERS spectra of the DMAB molecule showing overlay of on and off state for regions labelled a,b,c,d,and e.

7.5. (A) Raman map showing cone shape pattern drawn on the basis of intensity of each squares, and (B) SEM image of the same device showing cone shape structure of the Ag NP chain. 184

7.6. Current-time plot of the sample showing chain formation at the indicated voltage.

7.7. (A) Dark-field image of the Au/Ag NP chain/Au device, (B) Dark-field light scattering spectra at different locations in the 1D chain and (C) Normalized spectra of (B) 


\section{LIST OF TABLES}

TABLE

PAGE

1.1. Comparison of current and emerging memory technology based on its functions and properties.

3.1 Statistical data on the nanorods/nanowires grown by electrochemical seedmediated growth as a function of potential and time. 76

5.1. Summary of the humidity experiment showing humidity and voltage required to form chains at different $\%$ of humidity. 129 


\section{CHAPTER I}

\section{INTRODUCTION}

\subsection{Main Goal and Summary}

The original goal of the dissertation was to synthesize metal NWs/NRs onto electrode devices electrochemically in order to study their electronic properties and use them for chemiresistive sensing applications. During these studies, we accidentally discovered a method for electrochemical fabrication of onedimensional (1D) Ag nanoparticle (NP) chain structures in air that led to the greater part of this dissertation. After fabrication of Ag 1D chains, we studied applications, such as resistive switching (nanoelectronics), surface-enhanced Raman spectroscopy (SERS) combined with conductivity, and localized surface plasmon resonance (LSPR).

My dissertation work is organized as follows: Chapter I describes the background information about all the topics related to this research. Chapter II describes the chemicals, the experimental procedures, and the instrumentation that was used in this research. Chapter III describes the synthesis of $1 \mathrm{D}$ Ag NWs/NRs directly onto electrode surfaces electrochemically using a seed-mediated growth procedure. These experiments led to the discovery of the Ag NP chain formation, which took the research into a different direction. Chapter IV describes the 
synthesis and mechanism of forming $1 \mathrm{D}$ Ag nanochains spanning across $5 \mu \mathrm{m}$ gap electrodes. Chapter V highlights the switching properties of the 1D Ag nanochains spanning across microgap electrodes. Chapter VI explores the analytical applications of the 1D chain for combined electronic and optical measurements. We combined surface enhanced Raman spectroscopy with electronic measurements of the 1D nanochains in order to understand the effect of conductance on the Raman enhancement of 1D coupled Ag nanoparticles (NPs). Chapter VII describes a method for fabricating two terminal resistive switches using Ag NW-based junction contacted with polyphenol. Chapter VIII includes a summary and conclusions, as well as future directions of this research.

\subsection{Motivation /Objective}

The initial objective of the research was to synthesize Ag NWs/NRs across 5 $\mu \mathrm{m}$ gap electrodes and study their sensing applications. In order to develop this method, we first electrochemically synthesized Ag NWs/Ag NRs onto glass/ITO electrode surfaces using a seed-mediated growth procedure. Using our conditions $\mathrm{Ag} \mathrm{NWs/NRs} \mathrm{grow} \mathrm{with} \mathrm{the} \mathrm{average} \mathrm{length} \mathrm{ranging} \mathrm{from} 100 \mathrm{~nm}$ to $3 \mu \mathrm{m}$. We used the same method of synthesis to grow Ag NWs/NRs electrochemically across $5 \mu \mathrm{m}$ gap electrodes and found no Ag NW/NR formation across the gap. The device was then taken and potential was applied in air. After few scans from 0 to 5 V, device current showed 9 orders rise in magnitude. From the microscopy results, we observed Ag NP chains instead of Ag NWs/Ag NRs across $5 \mu \mathrm{m}$ gap electrode. This study led us to discovery of $1 \mathrm{D}$ Ag NP chains across the $5 \mu \mathrm{m}$ gap electrode. 
After this discovery, our primary motivation was to study the formation mechanism of the chain formation and resistive switching mechanism. We electrochemically deposited Ag nanostructures onto $5 \mu \mathrm{m}$ gap Au interdigitated array (IDA) electrodes or a $5 \mu \mathrm{m}$ gap Au two-electrode (TED) in the presence of surfactant cetyltrimethylammoniumbromide (CTAB), which led to Ag NP chains on the IDA or TED electrodes under the applied potential. Our procedure is simple, easy, requires minimal sample preparation and template-free and is unique in comparison to other methods reported as the chains are formed in air. After the chain formation across the electrode gap, device showed interesting resistive switching properties. The device could be made to switch from high resistance state (HRS) to low resistance state (LRS) under the applied potential. This type of resistive switching behavior shows application in building memory devices.

Our group previously used an electrochemical approach to grow 1D Ag NWs across electrode gaps in order to form electrode/Ag NW/polymer film/electrode junctions. We used the same approach to study the resistive switching properties of Ag NW-based electrode junctions.

Another motivation of this study was to understand optical properties of 1D Ag NP chains in order to explore applications in plasmonics. Ag 1D NP chains could act as a good SERS substrate for sensing Raman active molecules. SERS is especially used for spectroscopic measurements at nanogap metal electrodes since the metal can enhance the Raman signal. This study will motivate us to perform combined SERS and conductance measurement since our device could be made to switch from high resistance (HRS) to low resistance state (LRS) and vice versa. 
Further, metal NPs such as Ag and Au possess free conduction electrons which will generate surface plasmons when electrons interact with the electromagnetic waves of light. Our objective was to study the effect of current flowing through the assemblies on plasmon coupling and SERS properties, which will help in understanding the electronic properties of the Ag NP chains by correlating with the changes in optical properties of the Ag NPs under the applied potential.

\subsection{D Metal Nanostructures and their Importance}

Nanostructures - structures that are defined as having at least one dimension between 1 and $100 \mathrm{~nm}$ are of great importance as they exhibit very interesting and new phenomena. According to the number of dimensions less than $100 \mathrm{~nm}$, nanostructures can be classified into two-dimensional (2D, nanofilm), 1D (NWs, NRs) and 0D (NPs) structures. In particular, 1D nanostructures have attracted much attention due to its unique electrical and optical properties. ${ }^{1}$ They play an important role in fabricating electronic, ${ }^{2}$ optoelectronic, ${ }^{3,4}$ plasmonic,, 5 electrochemical, and electromechanical devices with nanoscale dimensions. ${ }^{7}$

\subsection{Synthesis of 1D Metal Nanostructures}

The synthesis of 1D metal nanostructures such as wires, rods, belts, and tubes has become a focus of intensive research owing to their unique applications in fabricating nanoscale devices. ${ }^{7,8}$ In general, the synthesis involves a metal ion precursor $\left(\mathrm{M}^{\mathrm{n}}\right)$ which is reduced to $\mathrm{M}^{0}$ in the presence of shape-inducing agents. ${ }^{9,10}$ Simple reduction of metal salts generally results in the formation of spherical NPs. ${ }^{11}$ By suitably controlling the experimental parameters such as 
concentration of the metal precursor, reducing agents, and stabilizers and reaction conditions such as temperature, time, and so on, it is possible to tune the size and shape of the NPs. Many methods have been developed to synthesize 1D metal nanostructures. Some of the methods used commonly are seed-mediated method, polyol method, electrochemical method and template-mediated method. ${ }^{1,10}$

\subsubsection{Template Directed Synthesis}

In this approach, a template serves as a scaffold to generate 1D nanostructures within the template. Several templates have been successfully demonstrated to aid synthesis of 1D nanostructures on surfaces such as step edges present on the surfaces of a solid substrate, channels within a porous material or biological macromolecules such as DNA strains or rod-shaped viruses. Penner and co-workers used an electrodeposition method to grow metal NWs on the step edges of highly oriented pyrolytic graphite where metal NWs preferentially nucleate and grow along the step edges on a graphite surface. ${ }^{12}$ Nanoscale channels within a porous material are another class of templates most commonly used developed by Martin and several others. ${ }^{13,14}$ Ordered alumina and polycarbonate membranes are the most widely used porous materials. ${ }^{13,15}$ In both the cases, the nanostructures resulted in well-aligned arrays within the pores and then released from the template and collected in the free-standing form. Carbon nanotubes (CNTs) have also been exploited to generate NRs or NWs. ${ }^{16}$ In comparison with porous membranes, it is difficult to remove NWs from CNT templates when needed for electronic applications. 


\subsubsection{Polyol Synthesis}

Fievet et al and Viau et al developed this method to make colloidal particles of various shapes and sizes made of metals and their alloys. ${ }^{17}$ The polyol synthesis involves the reduction of an inorganic salt precursor by polyol at an elevated temperature. Polyol refers to alcohols containing multiple hydroxyl groups such as Ethylene glycol, propylene glycol, pentane diols, glycerols, and so on. Polyol syntheses have been used to produce a variety of nanostructures such as cubes, rods, wires, or spheres by adjusting the relative amounts of the capping agent and the metal precursor ions in solution. ${ }^{18-20}$

\subsubsection{Seed-Mediated Synthesis}

Seed-mediated growth method has emerged over the past decade as the most common chemical method to synthesize a variety of nanostructures directly on substrates. It was originally used to synthesize spherical Au NPs of various size. ${ }^{21}$ Later this method was used for the preparation of Au NRs in solution developed by Murphy and coworkers. ${ }^{22}$ The method involves the chemical reduction of $\mathrm{AuCl}_{4}^{-}$by weak reducing agent (ascorbic acid in this case), in the presence of CTAB onto preformed Au NP seeds in aqueous solutions. 1D NRs of $\mathrm{Ag}^{23}$ and

$\mathrm{Cu}^{24}$ have also been prepared using seed-mediated growth in aqueous solution.

One of the obvious interests lies in developing a method to produce 1D nanostructures on surfaces for its future use in electronics. Taub et al described a strategy to form Au NW arrays on mica and silicon surfaces using seed-mediated 
growth. ${ }^{25}$ Later, our group synthesized Au NRs/NWs directly onto glass surfaces using seed mediated growth process in the presence of CTAB. ${ }^{26-29}$ Similarly, Ag NRs/NWs were also synthesized directly on surfaces using seed-mediated growth procedure. ${ }^{30}$ All these examples are involves the direct growth of 1D metal NRs on non-conductive surfaces using ascorbic acid as the chemical reducing agent and shape directing agent (CTAB in this case). $\mathrm{CTAB}$ acts as a bilayer that strongly adsorbs along the long axis of the crystal face of the Au NR, allowing the growth to occur preferentially on the ends of the NRs.

1D nanostructures are also synthesized on surfaces using an electrochemical approach. Tian et al described the shape control of $\mathrm{Au}$ electrodeposited on an $\mathrm{Au}$ film for the first time. ${ }^{31}$ A one-step, non-templated, low-cost electrochemical method was described for the growth of pyramidal, rod-like, and spherical Au nanostructures on polycrystalline Au substrates at different potentials and different $\mathrm{HAuCl}_{4}$ concentrations. Our group used a different strategy to electrodeposit $\mathrm{Au}$ nanostructures onto glass/ITO surfaces using seed-mediated growth. ${ }^{29}$ For the first time, the effect of the reduction potential was studied on the growth $\mathrm{Au}$ nanostructures in the presence of $\mathrm{CTAB}$ as the surfactant. Figure 1.1 illustrates the seed-mediated growth to form 1D metal nanostructures of different shapes. 

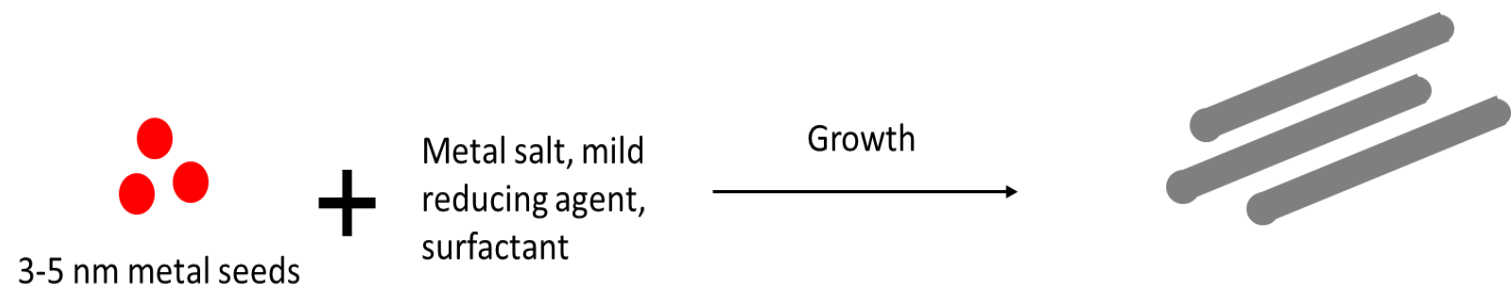

Figure 1.1. Illustration of the seed-mediated growth method. 


\subsubsection{Electrochemical Synthesis}

This method is versatile as a wide variety of materials such as metals, metal oxides, alloys, and polymers can be electrodeposited onto substrates directly. Reetz and Helbig demonstrated size-selective synthesis of tetraalkylammoniumstabilized Pd and Ni clusters by a simple electrochemical process. ${ }^{32}$ Au nanocubes and Au nanorods were also synthesized electrochemically by using a capping agent CTAB as it preserves the shape and stabilizes the nanostructures. ${ }^{33,34}$ All these examples uses a two electrode cell set up where Au plate is used as a anode and $\mathrm{Pt}$ plate used as a cathode and 1D nanostructures are synthesized in solution. Further, 1D nanostructures were also synthesized on surfaces using a three electrode cell set up using a Pt foil counter electrode, saturated calomel reference electrode and a glassy carbon working electrode. Pt nanothorns were electrodeposited on glass carbon electrode using a square wave potential technique. ${ }^{35}$

In order to fabricate an electronic devices, 1D nanostructures have to be electrodeposited between contact electrodes for its future application in nanoelectronics. Penner and co-workers describes a wire-based sensor to fabricate Pd mesowires electrochemically from aqueous solutions of $\mathrm{Pd}^{2+}$ onto step edges present on a graphite surface. ${ }^{36}$ Later they employed electron-beam lithography (EBL) technique to make contacts using Pd NWs. Our group developed an electrochemical method to grow 1D metal nanostructures directly onto electrode gaps. $\mathrm{Pd} \mathrm{NWs}{ }^{37}$ and $\mathrm{Ag} \mathrm{NWs}{ }^{38}$ were electrodeposited on to $5 \mu \mathrm{m}$ gap interdigitated array (IDA) electrodes. The method is simple, highly reproducible 
electrochemical method where 1D metal nanostructures are directly inserted between contact electrodes, hence eliminating the need for multistep processes involving transfer, assembly, and lithography.

In our dissertation work, we used seed-mediated and electrochemical method as explained in section 1.4.3 to grow 1D Ag NWs/NRs on glass/ITO surfaces. Chapter III explains the method in detail and discusses the optimum conditions to grow Ag NRs/NWs.

\subsection{Assembly of 1D Metal Nanostructures}

Metallic NPs and their assemblies have attracted extensive attention, particularly in nanotechnology because of their unique properties compared to the bulk. Linear or chain-like assemblies of metal NPs are another class of 1D nanomaterials. Various parameters play an important role in assembling NPs in an ordered manner. Metal NPs and their assemblies are classified into three types as shown in Figure 1.2. The assembly of NPs into 1D array on surfaces is of interest for the development of magnetic memory, switching, and sensing devices. Organization or self-assembly of NPs into multiple dimensions-for instance, chains, wires, spirals, etc.- provides optical and electrical properties that are distinct compared to individual NPs. These assemblies find potential applications in areas such as plasmonics, ${ }^{39}$ electronics,${ }^{40}$ and sensing. ${ }^{41}$ 


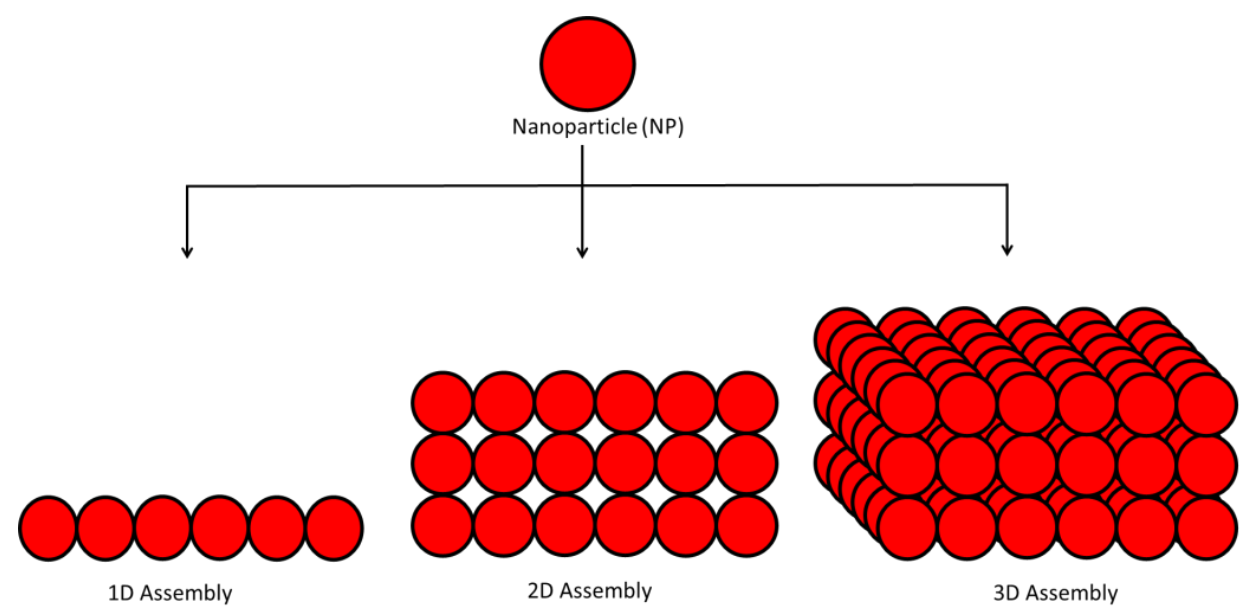

Figure 1.2. Scheme showing arrangement of metal nanoparticles into 1D, 2D and 3D assemblies. 


\subsection{Synthesis of 1D Assemblies}

1D NP assemblies can be synthesized using a number of techniques/methods as explained in several review papers. ${ }^{1,42-49}$ The organization of NPs in nanomaterials and nanodevices can be accomplished by top-down or bottom-up methods. ${ }^{49-51}$ A top-down approach commonly refers to the sculpting or etching of a bulk material to create nanoscaled structures/ functional devices with the desired shapes and characteristics. Various methods of lithography are used in the top-down approach. A recent review by Lifeng Chi et al focuses on lithography techniques. ${ }^{52}$ The most common techniques are focused on ion beam lithography (FIB) ${ }^{53}$ and electron-beam lithography (EBL). ${ }^{54-57}$ The bottom-up method involves the formation of 1D nanomaterials from atomic or molecular precursors in solution, followed by their assembly on substrates. Nanoparticles produced by top-down approaches have a relatively broad size distribution, varied particle shape or geometry, and use expensive synthetic pathways that are industrially nonscalable. ${ }^{58}$ Bottom-up methods appear to be more promising because they mainly utilize the self-assembly of NPs- process by which NPs spontaneously organize into ordered structures. Bottom-up approaches are far more popular in the synthesis of NPs, hence we will discuss different methods in detail.

\subsubsection{Self-Assembly and Directed Assembly}

Self-assembly is the process by which NPs organize into ordered, macroscopic structures, either through direct interactions, or indirectly using a template or an external field. Self-assembly provides a simple and low-cost 
method for producing ensembles of particles in a controlled manner. Directed assembly is a process whereby self-assembly of NPs is triggered using directing agents, external fields, or templates.

Several templates have been used to produce 1D assembly. Proteins, DNA and biopolymers are called soft templates and carbon nanotubes (CNTs) are called as hard templates. Directed assembly of NPs was first reported using soft template by Schultz et al where functionalized Au NPs selectively attached to sulfhydryl groups on the DNA strand. ${ }^{59}$ Later Kiehl et al also demonstrated the use of DNA nanogrids to assemble Au NPs in linear arrays on a solid surface. ${ }^{60}$ DNA templates are more commonly used. Use of peptide fibrils, ${ }^{61}$ cellulose ${ }^{62}$ are some other biomolecules that could mediate assembly of nanaoparticles into 1D assemblies Au, Ag or Pt. Directed assembly using hard template such as CNTs have been used to form 1D assemblies. One example is Au NPs attached to the outer surface of CNTs through a hydrophobic interaction. ${ }^{63}$ Rigid sheets of amorphous carbon, Zeolite-type materials are some of the other hard templates that have been used.

NPs can spontaneously organize via non-covalent repulsive and attractive interactions between them without the use of templates. Interparticle forces between adjacent NPs play an important role in producing $1 \mathrm{D}$ assemblies using template-free assembly. Linear aggregation of Au NPs into closely linked chains were formed on silicon substrates via dipole-dipole interactions. ${ }^{64}$ Linker molecules also provide the ability to produce 1D assemblies. Some of the examples of linker molecules are alkanedithiol molecules, amide linkages, biotin 
disulfide and streptavidin. 1D Au NP chains were prepared by covalent attachment of citrate-stabilized Au NPs to polymer via amide bond. ${ }^{65}$ One of the problems of self-assembly is aggregation of NPs. To prevent aggregation, several methods have been developed; among them is coating NPs with surfactants where one end of the surfactant chain is strongly attached to the surface and other end is free. Use of surfactant CTAB plays an important role in determining the nature of agglomeration. Murphy studied the role of CTAB in self-assembly and explained that surface-bound CTAB molecules provides steric as well as electrostatic repulsion between adjacent NPs where NPs preferentially bind with another by sharing a common layer of counter ions or through the interdigitating of CTAB tails. ${ }^{66} 1 \mathrm{D} \mathrm{Au}$ NP chains were prepared using $\mathrm{CTAB}$ where one end of the CTAB molecule preferentially binds to two (100) facets of the Au NP and the free end of CTAB molecule serves as the 'glue' that can link other adjacent Au NPs via electrostatic interaction. ${ }^{67}$

\subsubsection{Assembly by External Forces}

Optical, magnetic and electric fields have been widely used to form 1D assemblies. Magnetic NPs tend to align their magnetic moments in the direction of the magnetic field resulting in the formation of chains ${ }^{68}$ Light-assisted assembly of NPs depends on optical techniques or irradiation-induced changes in photoactive ligands. ${ }^{69,70}$ Organization of NPs into chains occurs under an electric field as well. Several groups have reported on the use of electric fields to form 1D assembly across two separated metal electrodes. Electric field induced 
polarization of NPs causes interaction with the adjacent NPs, resulting in the formation of chains aligned parallel to the field lines. Both alternating current (a.c.) and direct current (d.c.) have been used for NP assembly. ${ }^{71,72}$ One of the most versatile methods for nanoparticle assembly is dielectrophoresis (DEP). ${ }^{73-75}$ DEP can be understood by considering the polarizable object placed in a nonuniform electric field. DEP has been extensively studied for trapping Au NPs that results into assemblies of Au NPs into nanogap electrode. Stellacci et $a l^{75}$ explained the DEP technique showing that there are several parameters for particle assembly. These parameters are ac voltage, gap size, frequency, time and more important electrostatic interactions between particle and the substrate. AC electric fields are also used to assemble 1D structure from metallic NPs. ${ }^{76-78}$

\subsubsection{Assembly by Biomolecule Linkers}

Biomolecules such as DNA, proteins, are commonly used templates for the synthesis of 1D NP assemblies in solution. Self-assembly of metal NPs into linear superstructures is formed when functionalized metal NPs carry positive charge interacts with the negatively charged phosphate backbones of DNA molecules.

Different kinds of NPs, such as $\mathrm{Ag},{ }^{79,80} \mathrm{Pd},{ }^{81} \mathrm{Au},{ }^{82} \mathrm{Pt}^{83}$ have been organized using this technique through electrostatic interactions. Besides DNA, linear fibrous biomacromolecules like fibrin, ${ }^{61}$ dextran ${ }^{84}$ and collagen ${ }^{85}$ have been used to form 1D assemblies. 


\subsubsection{Assembly by Interparticle Forces}

Interparticle forces provide an excellent opportunity for NPs to organize into $1 \mathrm{D}$ or $2 \mathrm{D}$ or $3 \mathrm{D}$ nanostructures. Covalent, hydrogen bonding, electrostatic, hydrophobic are some of the interactions involved between NPs that helps in forming $1 \mathrm{D}$ assemblies in solution. The interaction between Au NR and alkanedithiol of varying chain lengths leads to the longitudinal assembly of Au NRs studies by Shibu Joseph et al. ${ }^{86}$ The end-to-end assembly was formed via covalent bonding of two thiol groups for form a disulphide. Linear assemblies of Au NRs can be formed through intermolecular hydrogen bonding. Au NR is coated with carboxylic acid derivatives, namely, 3-mercaptopropionic acid (MPA) and 11-mercaptoundecanoic acid (MUA), where thiol group binds to the surface of Au NRs and the appended carboxyl groups assist in the self-assembly of NRs through intermolecular hydrogen bonding for assembling Au nanorods. ${ }^{87}$ Electrostatic interaction is very common to form 1D assemblies. Electrostatic interaction between the amino acids, namely cysteine and glutathione containing thiol moieties anchored onto the Au NRs resulted in the formation of preferential longitudinal assembly of Au NRs in solution was studied. ${ }^{88}$ Assembly of Au NRs via hydrophobic-hydrophilic interactions was studied by Nie et $a l^{89}$ Au NRs functionalized with hydrophobic polymer on the ends and with CTAB along the long sides were stabilized in an organic solvent, after the addition of water to the organic medium, self-assembly was triggered by reducing the solubility of polymer coated $\mathrm{Au}$ NRs and further formed chains in solution. 


\subsubsection{Assembly on Templates}

Carbon nanotubes (CNTs) are commonly used templates due to their rigidity and toughness. CNTs have hollow structure; both the outside surface and inside surface can be used as templated for the deposition of NPs. Various metals have been used to form $1 \mathrm{D}$ assemblies using CNTs as templates. For example, $\mathrm{Au},{ }^{90}$ $\mathrm{Ag},{ }^{91} \mathrm{Pd},{ }^{92}$ and $\mathrm{Pt}^{93}$ are some of the metals. Ridged sheets of amorphous carbon have also been used to prepare chains of Au NPs with high aspect ratio. ${ }^{94}$

\subsubsection{Assembly on Surfaces}

Our group synthesized highly aligned Au NRs directly onto amine functionalized Si (100) through a surface chemical amidation reaction. ${ }^{95}$ Using this approach, 1D nanostructures can be further incorporated into nanoelectronic devices. Further, Au NRs were grown on patterned silicon substrates as described by Zhang and Imae. ${ }^{96}$ The combination of top-down surface patterning and bottom-up assembling was used for growing Au NR superlattices. The Au NR superlattices preferably deposited on the hydrophobic stripe region in the patterned substrate.

\subsubsection{Assembly on Electrode Gaps}

The directed assembly of NPs and nanoscale materials into specific locations is one of the major challenges in nanotechnology. The directed assembly will lead to fabrication of an electronic device. One method to assemble metal NPs is by using DEP. We discussed the DEP method in the section 1.6.2 in directed assembly by external forces. Another method is the electrochemical method. Tao 
and co-workers fabricated copper NWs across Au electrodes using an electrochemical method. ${ }^{97}$ Haynie and co-workers developed a method for assembling Pd NWs between microgap electrodes from a palladium acetate solution. ${ }^{98}$ Our group used electrochemical method to form 1D Ag NW across the $5 \mu \mathrm{m}$ gap IDA electrode. ${ }^{38}$ This method is very simple and easy to synthesize 1D metal nanostructures directly on surfaces.

Another class of nanomaterial is $1 \mathrm{D}$ arrays/assemblies/chains across the electrode gap, where metal nanostructures are deposited on an insulating surface and electrical contacts are made later. Researchers have formed conducting filaments $(\mathrm{CFs})^{99,100}$ or 1D chains of Ag across the electrode gaps by applying potential in presence of humidity. ${ }^{40} 1 \mathrm{D}$ arrays/chains across electrode gaps are also referred as conducting filaments (CFs) in most of the papers, where CF is an electronic term. The advantage of growing nanostructures directly on substrates lies in its convenience for its direct electrode contacts.

Using self-assembly (as explained in section 1.6.1) and electrochemical method (as explained in section 1.6.7), we synthesized 1D NP chains across $5 \mu \mathrm{m}$ gap electrodes. The method of synthesis is explained in detail in Chapter V.

\subsection{Two Terminal Resistive Switching in 1D Metal Nanostructures}

There has been increasing interest in the conductivity of metal-insulatormetal structures and various phenomena such as threshold switching, memory switching and negative differential resistance are observed through changes in electrical conduction. We will focus on memory switching and discuss its 
importance in detail. There are two types of memory in our computers: random access memory (RAM) and read only memory (ROM). ${ }^{101}$ The ROM memory is nonvolatile, meaning it does not lose its contents when there is no power, unlike random-access memory, better known as RAM, which is volatile. As a result, ROM is the preferred storage for important data in computers. Today's research is focused on building non-volatile memories (NVMs) that can overcome the problem of scalability in current NVMs. ${ }^{102}$ Most notably, NVMs based on resistive switching have attracted considerable interest and are termed as resistive random access memory (RRAM). Several review papers have discussed its importance in detail. ${ }^{103-106}$ There is a focus of research on RRAM for obtaining fast, high density, low-power consumption, and reliable memory. Current memory technology relies on dynamic RAMs (DRAMs) and flash memories for data storage and non-volatility. Flash memory, which is currently very popular, has very high capacity and is non-volatile, but is relatively slow. ${ }^{107,108}$ The disadvantages of flash memories are scalability and limited cyclability $>10^{5}$ write/erase cycles. The other emerging memories are ferroelectric RAMs (FRAMs), ${ }^{109}$ resistive RAMs (RRAMs), ${ }^{103,110-112}$ magnetic RAMs (MRAMs), ${ }^{113}$ phase change RAMs (PRAMs), ${ }^{114}$ electrochemical metallization (ECM) memories, or programmable metallization cell (PMC) memories. ${ }^{110}$ Table 1.1 provides a comparison between current and emerging memory technologies. Resistive switching memories are currently gaining wide interest with great potential as emerging NVMs. Resistance memories include two distinct resistance states called as the high resistance state (HRS) and low resistance state (LRS). 
Table 1.1. Comparison of current and emerging memory technology based on its functions and properties.

\begin{tabular}{|c|c|c|c|c|c|c|c|}
\hline & \multicolumn{4}{|c|}{ Current memory technology } & \multicolumn{3}{|c|}{ Emerging Non-volatile memory } \\
\hline & SRAM & DRAM & Disk & $\begin{array}{l}\text { Flash } \\
\text { memory }\end{array}$ & PCRAM & RRAM & MRAM \\
\hline Maturity & Produced & Produced & Produced & Product & Advanced & Early & Advanced \\
\hline Endurance & $>10^{15}$ & $>10^{15}$ & $>10^{15}$ & $10^{4}$ & $10^{8}$ & $10^{5}$ & $10^{15}$ \\
\hline Nonvolatile & No & No & Yes & Yes & Yes & Yes & Yes \\
\hline Speed & Fast & Slow & Slow & Slow & Slow & Fast & Fast \\
\hline Density & Low & Moderate & $\begin{array}{l}\text { Highly } \\
\text { dense }\end{array}$ & Low & Moderate & $\begin{array}{l}\text { Highly } \\
\text { dense }\end{array}$ & Low \\
\hline $\begin{array}{l}\text { Type of } \\
\text { material }\end{array}$ & $\begin{array}{l}\text { Magnetic } \\
\text { based }\end{array}$ & $\begin{array}{l}\text { Magnetic } \\
\text { based }\end{array}$ & $\begin{array}{l}\text { Optical } \\
\text { based }\end{array}$ & $\begin{array}{l}\text { Resistive } \\
\text { switching } \\
\text { based }\end{array}$ & & & \\
\hline
\end{tabular}


There are a number of materials that have been explored in fabricating nonvolatile memory devices. The device operates by an electrochemical mechanism, which leads to the formation and breaking of conducting filaments (CFs) as the resistive switching process. CF-based devices are classified into two types: redox processes induced by cationic migration or redox processes induced by anionic migration. ${ }^{103}$ Figure 1.3 shows two types of examples for cationic and anionic migration. The $\mathrm{Pt} / \mathrm{Ag}_{2} \mathrm{~S} / \mathrm{Ag}$ system is an example of cationic migration and the $\mathrm{Pt} / \mathrm{TiO}_{2} / \mathrm{Pt}$ system is an example of anionic migration. Redox processes by cationic migration involves oxidation of metal atoms at the anode that migrate towards the cathode where they are reduced. The reduced metal ions form a filament which grows towards the anode and turn the device to the ON state once the filament connects the cathode and anode. The system is mainly made up of active electrode $(\mathrm{Cu}$ or $\mathrm{Ag}$ ) and an inert electrode ( $\mathrm{Pt}$ in most cases) and an ionic conductor is present in between the two electrodes. ${ }^{115}$ The ionic conductors are made up of chalcogenides mainly and formed by dissolving the electrochemically active metal, $\mathrm{Ag}$ or $\mathrm{Cu}$, in sulfides, selenides, or tellurides of $\mathrm{Ge}, \mathrm{As}, \mathrm{Sb}$ or $\mathrm{Ga}$. The added $\mathrm{Ag}$ or $\mathrm{Cu}$ to the chalcogens form mobile cations and the mobility of these cations is very high which helps in formation of CF. Ag types CFs are common since it is cheap and abundant. ${ }^{16,117}$ In this system, metal cations in the ionic conductors migrate towards the cathode (made up of inert material) and get reduced. The reduced metal atoms form a metallic path and form connection with the anode to form CF. Several ionic conductors are used that are cation conductors for the redox active species such as $\mathrm{Ag}^{+}$in $\mathrm{Ag}_{2} \mathrm{~S},{ }^{118} \mathrm{Ag}^{+}$in $\mathrm{GeSe}_{\mathrm{x} .}{ }^{115}$ 




\section{B}
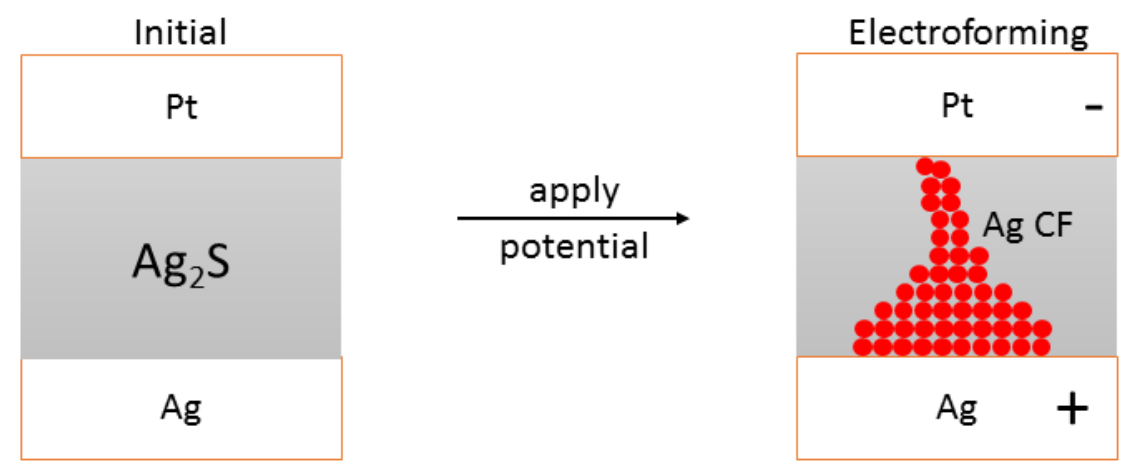

$$
\mathrm{Ag}^{+}\left(\mathrm{Ag}_{2} \mathrm{~S}\right)+\mathrm{e}^{-} \stackrel{\text { Reduction }}{\stackrel{\text { Oxidation }}{\rightleftarrows}} \mathrm{Ag}
$$

Figure 1.3. CFs formed by (A) anionic migration involving migration of oxygen vacancies and (B) cationic migration involving migration of Ag ions. 
Redox processes by anionic migration are observed in metal oxide based switching systems where oxygen vacancies migrate towards cathode. Oxygen vacancies are more mobile than cations which build and expand towards the anode. ${ }^{103}$ This system consists of two inert electrodes (mainly, Pt) having metal oxide in between the two electrodes. The formation and rupture of a CF is the major switching mechanism in many oxide systems. The most studied oxides are $\mathrm{NiO}^{119}$ and $\mathrm{TiO}_{2} .{ }^{120}$ The thermally and/or electrically activated motion of these native dopants results in chemical changes in the oxides, such as valence state change, leading to resistance switching. In $\mathrm{TiO}_{2}$-based switching system, insulating oxide contains native dopants that are resulted from oxygen deficiency or oxygen excess. On application of potential, native dopants in insulating oxide undergoes valence state change leading to resistance switching. ${ }^{103,104,110}$ The reduction of metal oxide at the cathode turns the oxide into a metallically conducting phase, such as $\mathrm{TiO}_{2-x}$. This is termed as virtual cathode which will move to form a conductive path. ${ }^{121}$ At the anode, the oxidation reaction leads to the evolution of oxygen gas. Once the formation of $\mathrm{CF}$ is completed, resistive switching takes place through a redox reaction between the virtual cathode and the anode by formation and dissolution of the conductive path.

Further, it is important to understand the formation process of CFs in order to optimize the structure and enhance the performance of RRAM devices. In previous studies, the formation process of CFs has been directly demonstrated by several techniques. These techniques are transmission electron microscopy 
(TEM), ${ }^{100,122}$ scanning electron microscopy (SEM) ${ }^{99,123}$ and atomic force microscopy (AFM). ${ }^{124}$

After the chains (or filament) are formed, the device can be made to switch from LRS to HRS repeatedly. The conductive path between the two electrodes is thought to be the formation of metal atomic bridges at the Ag NP junctions in the chain. This type of switch is called an atomic switch. ${ }^{125,126}$ Atomic switches are nanoionic devices that consist of the formation and dissolution of CFs via a solid electrochemical reaction that leads to major changes in the resistance between electrodes- the 'ON' and 'OFF' states Atomic switches are divided into two types of switches: Gap-type atomic switch and Gapless-type atomic switch. The gapless-type atomic switch differs from the gap-type atomic switch in that the inert electrode (usually Pt electrode) is in contact with an ionic conductive material. The gapless-type atomic switch has been demonstrated using several materials such as metal sulfides, ${ }^{127,128}$ chalcogenides,${ }^{129}$ or transition metal oxides. ${ }^{130}$ The redox processes i.e., reduction/oxidation of metal cations/atoms, makes both the gapless-type and gap-type atomic switches non-volatile. Metal cations/atoms are responsible for the switching operation in cation-based ionic devices, referred to as atomic switches. Chapter V discusses the resistive switching mechanism where the device could be referred to as atomic switch after formation of Ag NP chain across the microgap electrode.

Further it is also important to know where the LRS and HRS takes place within the filament region. Lui et al studied growth of conducting filaments for the two-terminal metal/insulator/metal system that consists of an active electrode 
(Ag) and an inert electrode (Pt) sandwiched and an dielectric film is sandwiched between the electrode. ${ }^{131}$ In their studies, they also observed the formation and dissolution of metallic filaments in the conduction path at the ON and OFF states. They performed TEM studies and found that the dissolution of the conducting filament occurs near the inert electrode (cathode). All filaments formed in the device were found to first break at the filament/inert electrode interface. Thus, they concluded that the thinnest region of the filament is expected to take part during switching cycles. ${ }^{132}$

CF-based devices can be used to develop memory devices. Before the application, there are several features or parameters that needs to be understood and examined. Waser et $a l^{110}$ have discussed these features in comparison with existing flash memory. The current features of existing flash memory shows endurance of $10^{3}$ to $10^{7}$ cycles, resistance ratio (ON/OFF ratio) $>10$ and programming speed of $10 \mu \mathrm{s}$ to $25 \mu \mathrm{s}$. Our device shows considerably good endurance behavior $>10^{3}$ cycles, resistance ratio $>10$ and moderate programming speed of $25 \mathrm{~ms}$. Looking over these features, our device could be used in future for building memory devices.

We studied the resistive switching properties of 1D Ag NP chains formed across microelectrode gaps in air. After the formation of chains, we carried out IV scans for stable and reproducible switching behavior and later performed I-t plots to study the endurance, switching speed and resistance ratio. Our devices show endurance $>10^{3}$, switching speed of at least $0.0025 \mathrm{~s}$ and resistance ratio $\geq$ 10. In general, switching studies of CFs involve electroformation of filaments, 
stable and reproducible switching through I-V scans, and evaluation of the memory properties such as endurance, resistance ratio and switching speed as reported in the literature. ${ }^{116,118,133,134}$ The CF-based switching devices shows overwhelming advantages in easy fabrication, simple structure, excellent scalability, fast switching, high integration density, and shows good compatibility with the current complementary metal oxide semiconductor (CMOS) technology. ${ }^{107,135,136}$

A variety of solid electrolytes have also been used to fabricate resistive switching devices. We discussed some of the solid electrolytes such as chalcogenides and metal oxides. Polymers can also be used as a solid electrolyte. It generally consists of an insulating layer sandwiched between an electrochemically active electrode and an inert counter electrode. Resistive switches have also been fabricated using a polymer that has shown great interest Two terminal switches comprised of molecules or nanostructures, whose conductance can be switched between two states upon application of a voltage are highly pursued as they can be used for ultra-high density memory storage or logic devices. ${ }^{137}$ In general the resistive switch is fabricated where a resistive material is sandwiched between the two electrodes. Examples of resistive switch materials are organic polymers (polyphenol, polystyrene), ${ }^{38,138}$ chalcogenides (Ag-Ge-Se, $\mathrm{Ag}_{2} \mathrm{~S}$ and $\left.\mathrm{Cu}_{2} \mathrm{~S}\right),{ }^{139,140}$ metal oxides (Cu-doped $\mathrm{SiO}, \mathrm{WO}_{3}, \mathrm{ZrO}$, and $\mathrm{TiO}_{2}$, etc.) ${ }^{141,142}$ and amorphous $\mathrm{Si}(\mathrm{a}-\mathrm{Si}) .{ }^{143-145}$ Resistive switching in these devices has been explained in terms of the formation of conductive filaments in the insulating layer. The formation of a filament between two electrode gaps leads to low 
resistance state, and its rupture switches the device back to the high resistance state.

In our work, we fabricated metal/Ag NP chain/metal junctions which shows interesting applications in resistive switching. Chapter VI discusses the resistive switching properties and memory properties in detail. We also fabricated metal/polyphenol/Ag NW/metal junctions using previous method developed in our group and studies the resistive switching properties which will be discussed in Chapter IV.

\subsection{Simultaneous Measurements of Electronic Conduction and Raman Response in 1D Chains or CFs}

CFs shows interesting resistive switching properties. Thus, it is very important to understand how the filaments begin to grow during the ON state and how the conducting filament is disconnected during the OFF state. Wong et al investigated resistance switching mechanism in metal/oxide/metal based devices. Single layer graphene was inserted into the metal/oxide/metal system and oxygen movement was monitored by Raman spectroscopy. ${ }^{146}$ They observed noticeable changes in the D-band, G-band and 2D-band signals of the single layer graphene during electrical cycling. During consecutive electrical switching cycles, D band gradually decreases, but noticeable changes were observed for G-peak showing higher intensity at ON state and lower at OFF state while 2D-peak shows lower intensity at ON state and higher intensity at OFF state. Mc Creery and co-workers reported Raman spectroscopic measurements of organic polymer memory devices 
which permits direct monitoring of the doping state and conductivity of polythiophene in a 3-terminal device. ${ }^{147}$ A ferroelectric polymer (polythiophene) is used as an organic layer, where an electrical bias induced switching between two stable polarization states forms the basis for memory. The Raman spectral changes showed that there is a direct correlation between conductivity switching of polythiophene from neutral polythiophene to its polaron by showing higher intensity at ON state compared to OFF state. Very few papers have been reported for combined SERS and conductance switching of filaments. This work indicates that Raman spectroscopy could be a powerful and efficient method to investigate the RRAM mechanism.

Ag NP chain based device fabricated by us was taken and coated with 4-ATP (Raman active molecule). Since our device shows resistive switching properties, we carried out Raman measurements simultaneously with conductance switching under the applied potential. In Chapter VII we will discuss the combined SERS and conductance switching of our Ag NP chain device coated with 4-ATP.

\subsection{LSPR of 1D Nanochains}

It is very interesting to study the optical properties of 1D NP chains. There are several factors affecting the optical properties of a metal 1D chain..$^{5,148-153}$ The optical properties of metal nanoparticle chains not only depend on nanoparticle material, size and shape but also on the interparticle spacing and the number of

coupled NPs within a given chain. ${ }^{151,154-156}$ 1D chains across nanometric gaps generate strong plasmonic modes of Au as studied by Aizpurua et al. ${ }^{5}$ For a 
perfectly straight chain, a peak around wavelength $820 \mathrm{~nm}$ dominated the spectra corresponding to the lowest energy dipole-like mode. The incident electric field is parallel to the chain axis giving rise to longitudinal modes. Similarly Mulvaney et al examined the surface plasmon resonance in strongly coupled Au nanosphere chains. ${ }^{157}$ The plasmon bands are seen in the near infrared region for a 1D assembly. They also observed a strong plasmonic band for the nanoparticle chains arranged in a perfectly straight manner. Fery et al studied plasmonic modes of linear assemblies of Au NPs synthesized via template-assisted colloidal selfassembly. ${ }^{158}$ They compared the surface plasmon modes for monomer, dimer and tetramer chains of Au NPs. With an increasing number of chains, the strong plasmonic longitudinal dipolar mode was red shifted with peak-broadening from $800 \mathrm{~nm}$ to $1200 \mathrm{~nm}$. The strongly coupled plasmonic modes were observed for 1D assemblies of Au NPs that are uniformly arranged and coupled with each other in a chain. In Chapter VI, we will discuss the LSPR properties of individual 1D Ag NP chains formed electrochemically across $5 \mu \mathrm{m}$ gap electrodes. 


\section{CHAPTER II}

EXPERIMENTAL

\subsection{Chemicals and Solutions}

\subsubsection{Chemicals}

Cetyltrimethylammonium bromide $(\mathrm{CTAB}, \geq 97.0 \%)$, silver nitrate $\left(\mathrm{AgNO}_{3}\right.$, $\geq 99 \%$ ), trisodium citrate ( $\geq 99.0 \%)$, 4-aminothiophenol (4-ATP, 97\%), phenol (99\%), 1,8-octanedithiol ( $\geq 97 \%)$, sodium borohydride $\left(\mathrm{NaBH}_{4}, 98.0 \%\right)$, mercaptopropyltrimethoxysilane (MPTMS, $\geq 97.0 \%)$, isopropyl alcohol (99.8\%) and acetone (99.9+\%) were purchased from Sigma-Aldrich. Ethanol (200 proof) was purchased from Decon Laboratories, Inc. Sodium phosphate dibasic (98\%) was purchased from EMD chemicals and sodium phosphate tribasic (98\%) was purchased from Allied Chemicals. $\mathrm{H}_{2} \mathrm{SO}_{4}$ was purchased from VWR. NANOpure ultrapure water (resistivity of $18 \mathrm{M} \Omega-\mathrm{cm}$ ) was used for all aqueous solutions. $\mathrm{HAuCl}_{4}-3 \mathrm{H}_{2} \mathrm{O}$ was synthesized from metallic $99.99 \% \mathrm{Au}$ in our laboratory.

\subsubsection{Synthesis of 3-5 nm diameter Au nanosphere (NS) Seeds}

We used Murphy's procedure to prepare Au NP seeds. ${ }^{159}$ Small 3-5 nm diameter Au NSs, termed "Au seeds" were synthesized by adding $0.6 \mathrm{~mL}$ of ice cold $0.1 \mathrm{M} \mathrm{NaBH}_{4}$ to $19 \mathrm{~mL}$ of an aqueous solution of $2.5 \times 10^{-4} \mathrm{M}$ trisodium citrate and $2.5 \times 10^{-4} \mathrm{M} \mathrm{HAuCl}_{4} * 3 \mathrm{H}_{2} \mathrm{O}$ under stirring. The solution was used after stirring for at least 2 hours. 


\subsubsection{Aqua regia cleaning}

Aqua regia solution is a highly corrosive mixture of concentrated nitric acid $\left(\mathrm{HNO}_{3}\right)$ and hydrochloric acid $(\mathrm{HCl})$, with a volume ratio of $\mathrm{HNO}_{3}: \mathrm{HCl}=1: 3$. It is used for cleaning Au from the glassware and stir bars. The glassware and stir bars were put in fresh aqua regia solution for 30 minutes, and then rinsed thoroughly with deionized water and nanopure water for cleaning.

\subsection{Substrates}

\subsubsection{Microfabrication of $\mathrm{Au}$ electrodes on $\mathrm{Si} / \mathrm{SiO}_{2}$}

$5 \mu \mathrm{m}$ gap Au Interdigitated Array (IDA) electrode devices and $5 \mu \mathrm{m}$ gap $\mathrm{Au}$ Two Electrode Devices (TED) were used as substrates for depositing different metals, polymers, and molecules to fabricate $\mathrm{Au} / \mathrm{Ag} \mathrm{NP}$ chain/Au device for Surface Enhanced Raman Spectroscopy (SERS) and resistive switching studies. IDA and TED electrodes were microfabricated in a cleanroom using photolithography, sputtering, and liftoff in the University of Louisville Micro Nano Technology Center. Figure 2.1 shows the optical images of Au IDA and Au TED fabricated on $\mathrm{Si} / \mathrm{SiO}_{2}$.

\subsubsection{Microfabrication of Au electrodes on glass}

The Au TED on a borosilicate glass substrate was microfabricated in a cleanroom using photolithography, sputtering, and liftoff in the University of

Louisville Micro Nano Technology Center. $5 \mu \mathrm{m}, 10 \mu \mathrm{m}$ and $23 \mu \mathrm{m}$ Au TED were used as substrates for Ag deposition to fabricate Au/Ag NP chain/Au devices 

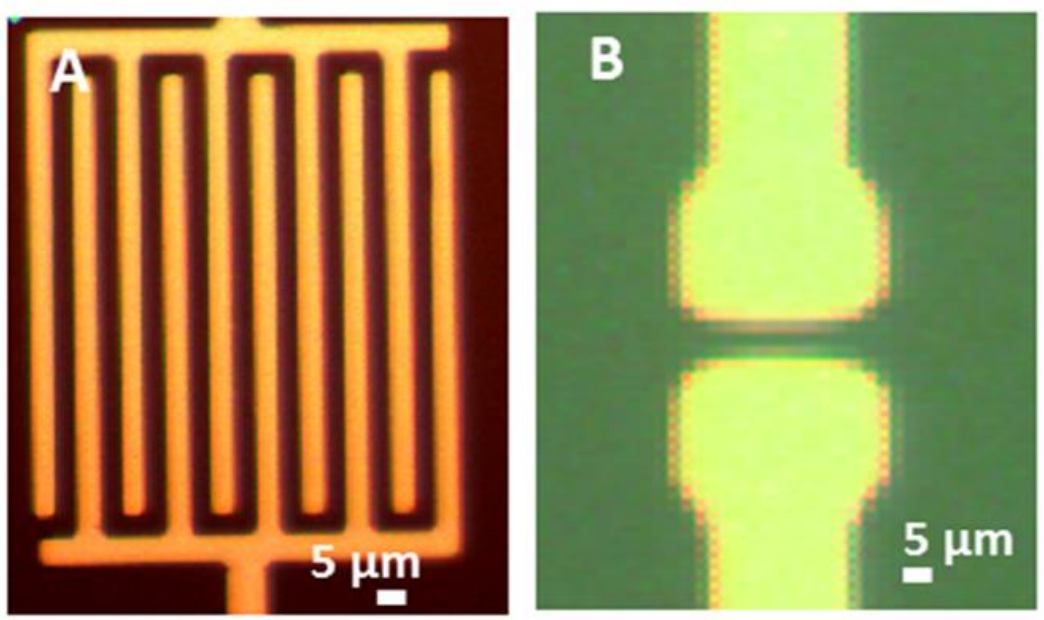

Figure 2.1. Optical images of bare $\mathrm{Au} 5 \mu \mathrm{m}$ gap (A) Au IDA device on $\mathrm{Si} / \mathrm{SiO}_{2}$ and (B) Au TED on $\mathrm{Si} / \mathrm{SiO}_{2}$. 
in particular for LSPR spectral analysis and dark-field imaging. Figure 2.2 shows the optical images of the $5 \mu \mathrm{m}, 10 \mu \mathrm{m}$ and $23 \mu \mathrm{m}$ gap Au TED fabricated on glass.

\subsubsection{Wiring and cleaning of the Au IDA or TED electrodes}

Figure 2.3 shows the steps involved in preparing the Au IDA or Au TED electrode either on glass or $\mathrm{Si} / \mathrm{SiO}_{2}$ for electrochemical studies. The two $\mathrm{Au}$ electrodes of the devices were cleaned by rinsing in acetone, ethanol, 2-propanol, nanopure water and drying under nitrogen. After drying, we carried out wiring of the electrodes as shown in Figure 2.3. After wiring, electrodes were again rinsed with different solvents such as acetone, ethanol, 2-propanol, nanopure water and

drying under nitrogen. After wiring and rinsing with different solvents, we placed the devices in an ozone chamber for 15 min to remove organic impurities. Then the electrodes were cleaned by electrochemical cycling in $0.1 \mathrm{M} \mathrm{H}_{2} \mathrm{SO}_{4}$ well defined Au oxidation and reduction peaks were observed. Figure 2.4 shows a tyical cyclic voltammogram of an Au IDA in $0.1 \mathrm{M} \mathrm{H}_{2} \mathrm{SO}_{4}$ with the oxidation and reduction peaks of $\mathrm{Au}$.

\subsubsection{Au IDA/TED electrode modification}

These substrates were used 1) directly after cleaning (termed "bare Au"), 2) after cleaning and placing in a $2 \mathrm{mM}$ ethanolic 1,8 -octanedithiol solution for $0.5 \mathrm{~h}$, rinsing with ethanol and water, and drying under $\mathrm{N}_{2}$ (termed "Au/dithiol”), or 3) after cleaning, functionalizing with 1,8-octanedithiol and soaking in a solution of 4 nm diameter Au NPs for 20 min, followed by rinsing with water and drying under $\mathrm{N}_{2}$ (termed "Au/dithiol/Au NPs"). The $4 \mathrm{~nm}$ diameter Au NPs were prepared as 

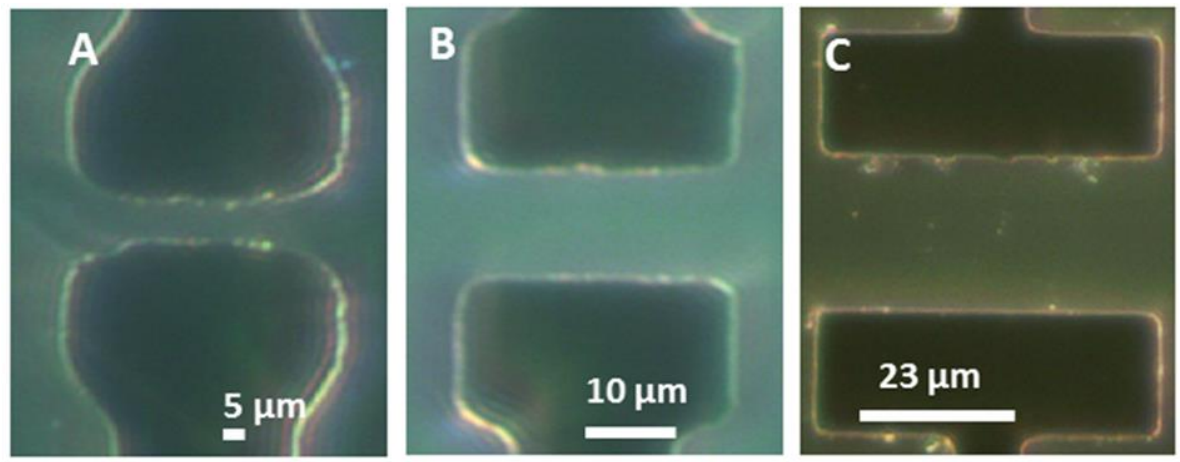

Figure 2.2. Dark-field microscopy images of Au TED with gap size of (A) $5 \mu \mathrm{m}$ Au TED, (B) $10 \mu \mathrm{m}$ Au TED and (C) $23 \mu \mathrm{m}$ Au TED. 

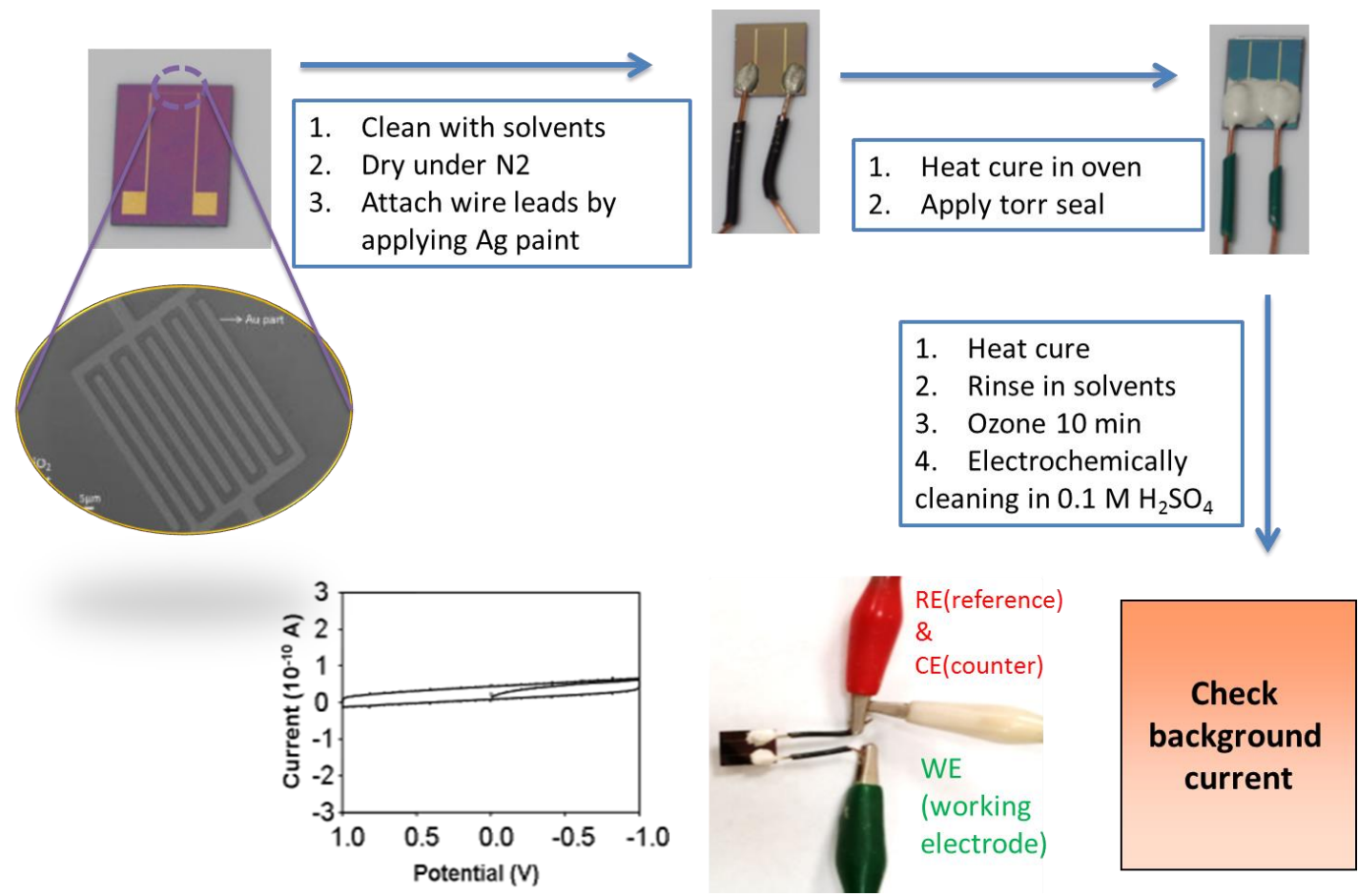

Figure 2.3. Steps involved in wiring and cleaning the electrode devices. 


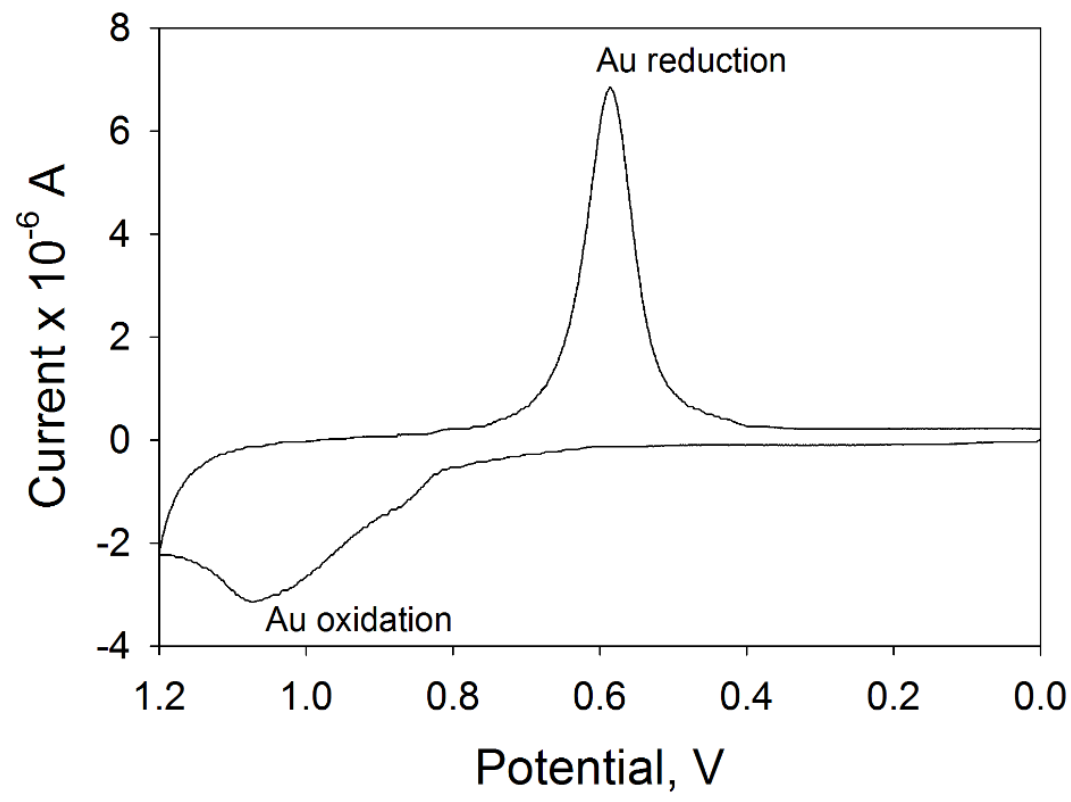

Figure 2.4. Cyclic voltmmogram of $\mathrm{Au}$ IDA in $0.1 \mathrm{M} \mathrm{H}_{2} \mathrm{SO}_{4}$ shows oxidation and reduction peaks of $\mathrm{Au}$. 
described by Murphy and co-workers. ${ }^{159,160}$

\subsubsection{Cleaning the glass/ITO electrode}

Single-sided $7 \mathrm{~mm}$ x $50 \mathrm{~mm}$ x $0.9 \mathrm{~mm}$ indium tin oxide (ITO)-coated glass (glass/ITO) electrodes with an 8-12 $\Omega$ resistance (Delta Technologies, Stillwater, $\mathrm{MN}$ ) were cleaned by sonication for $10 \mathrm{~min}$ each in nanopure water, acetone, and isopropyl alcohol sequentially.

\subsubsection{Glass/ITO electrode modification}

The cleaned glass/ITO electrode was functionalized by immersion for $20 \mathrm{~min}$ in a solution containing $10 \mathrm{~mL}$ of isopropyl alcohol, 1 to 2 drops of water, and 100 $\mu \mathrm{L}$ of mercaptopropyltrimethoxysilane (MPTMS) that was heated to just below boiling $\left(50-60^{\circ} \mathrm{C}\right)$. This led to a surface terminated with thiol groups. The glass/ITO/MPTMS was then rinsed and sonicated for $10 \mathrm{~min}$ in isopropyl alcohol and dried under $\mathrm{N}_{2}$ before being immersed in a solution of $4 \mathrm{~nm}$ diameter Au NPs for $20 \mathrm{~min}$, which led to the attachment of the Au NPs to the glass/ITO/MPTMS surface through strong Au-thiolate covalent interaction. The resulting glass/ITO/MPTMS/Au NP (4 nm) electrode was rinsed with nanopure water and dried under $\mathrm{N}_{2}$ before electrochemical studies. These electrodes were used for experiments involving the electrochemical seed-mediated growth of Ag NRs/NWs. Figure 2.5 shows the scheme of functionalizing the glass/ITO electrode. 


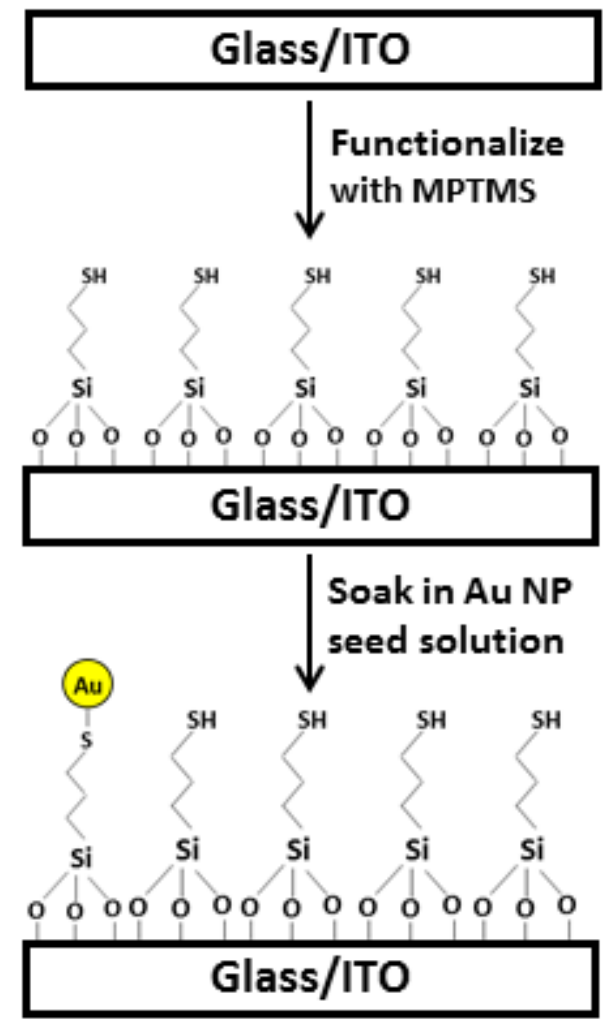

Figure 2.5. Scheme showing steps involved in fabricating glass/ITO/MPTMS/Au NP (4 nm) electrode. 


\subsection{Techniques}

\subsubsection{Electrochemistry}

All electrochemical measurements were performed using a $\mathrm{CH}$ Instruments 660A electrochemical work station using the following techniques:

Cyclic Voltammetry (CV): CV was used to:

1) Perform electrochemical cleaning using $0.1 \mathrm{M} \mathrm{H}_{2} \mathrm{SO}_{4}$.

2) Determine electrochemical deposition potentials for Ag on Au.

3) Characterize the Au electrodes before and after Ag.

4) Characterize the device after chain formation.

All CVs were performed at room temperature with $5 \mu \mathrm{m}$ gap, $10 \mu \mathrm{m}$ gap and $23 \mu \mathrm{m}$ gap electrodes, $\mathrm{Ag} / \mathrm{AgCl}(3 \mathrm{M} \mathrm{KCl})$ or $\mathrm{Ag}$ wire reference electrodes, and platinum wire counter electrodes. Figure 2.6 shows the setup used to perform all CVs in solution.

We also obtained CV s in air or I-V curves for measuring the resistance of nanochains. For this, we connected the reference and the counter electrode cables to one wire lead of the Au IDA and the working electrode cable to the other lead (see Figure 2.7 for setup). All I-V curves in air were obtained in a Faraday cage.

Chronocoulometry (CC): $\mathrm{CC}$ is an electrochemical technique in which the potential of the working electrode is stepped to a constant value and the number of coulombs passed at the electrode is monitored as a function of time. In our studies, we used this technique for the controlled deposition of Ag onto the Au electrodes. 


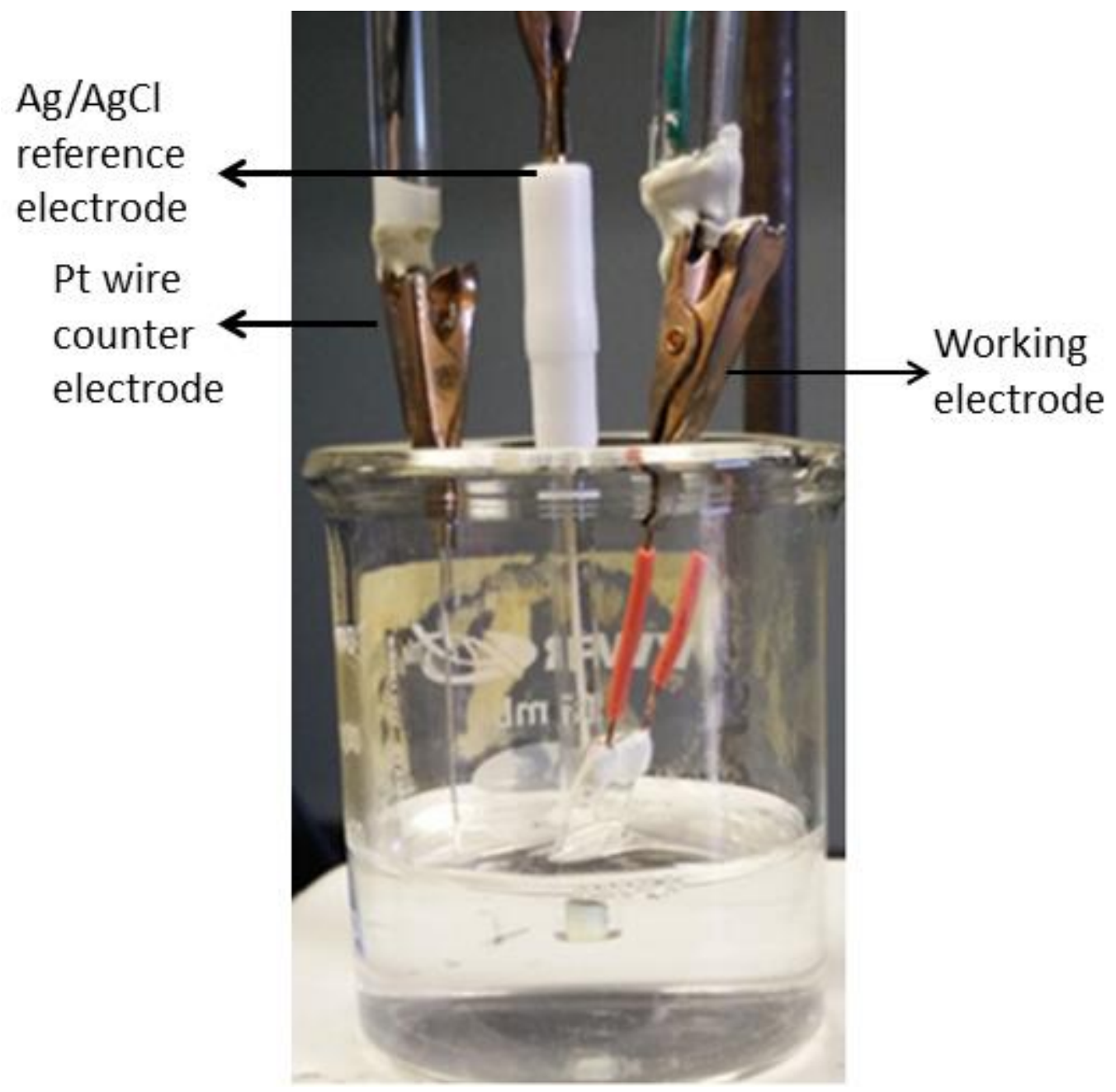

Figure 2.6. Digital photograph showing conventional three electrode set up for metal electrodeposition 


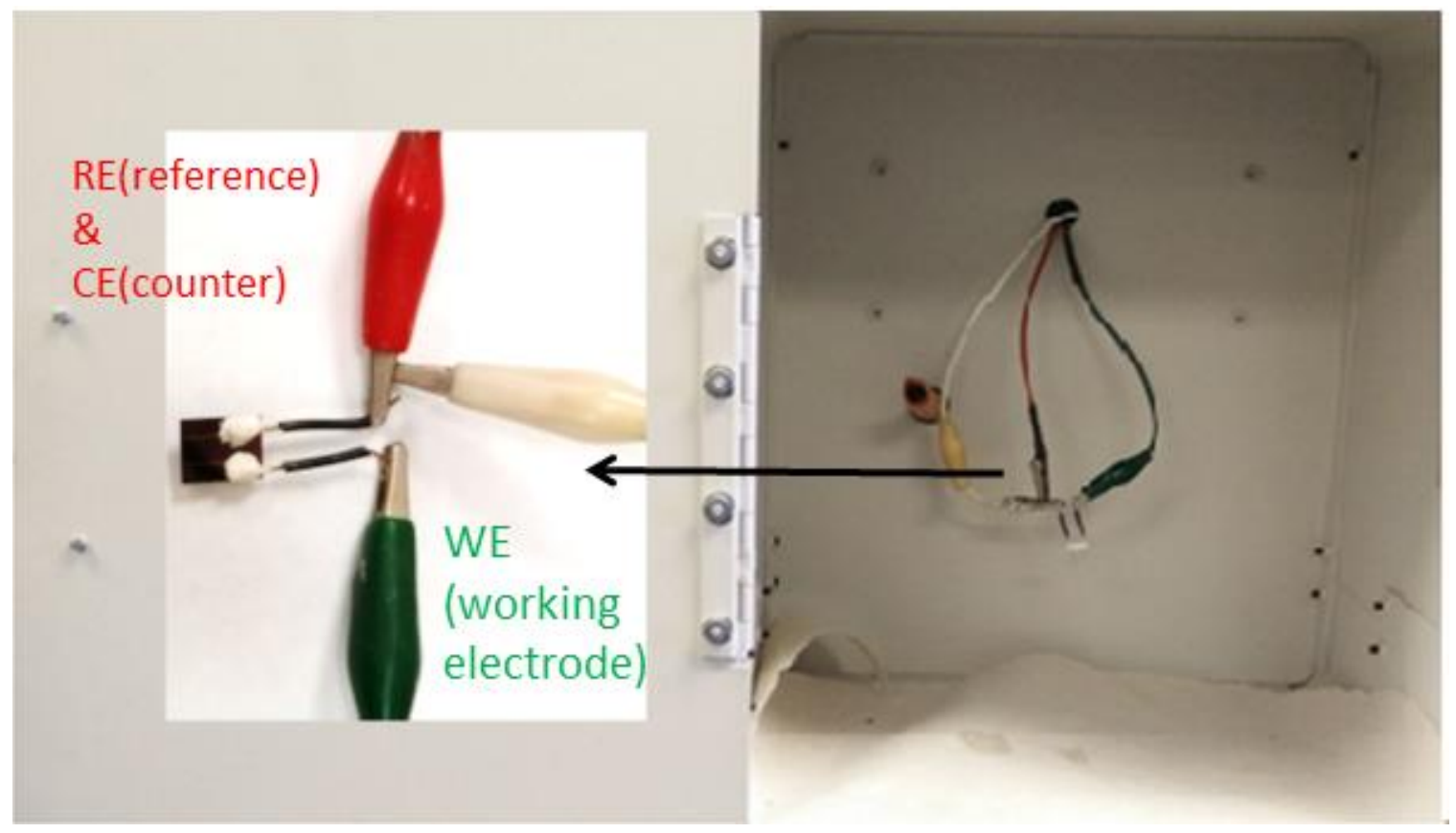

Figure 2.7. Digital photograph showing the two electrode setup for I-V or I-t measurements in air in CV or CA mode, respectively, of the CHI660A. 
The potential of the working electrode was stepped to a potential negative enough to reduce $\mathrm{Ag}^{+}$onto the $\mathrm{Au}$ IDA.

Chronoamperometry (CA): CA is an electrochemical technique where one steps the potential of the working electrode to a constant value and monitors the current as a function of time. The Ag nanoparticle chains were formed by holding the potential across the microelectrode IDA or TED gap and monitoring current as a function of time. The rise in a current indicated the chain formation. We later carried out endurance and switching speed studies by applying ON and OFF voltages at different pulse rates for different numbers of cycles using the chronoamperometry technique (current-time plots).

\subsubsection{Scanning Electron Microscopy (SEM)}

SEM images were obtained using a Carl Zeiss SMT AGSUPRA 35VP field emission scanning electron microscope operating at an accelerating voltage of 8.00 KV. SEM images directly provided information about the shape, size, and structure of the Ag NP chains and Ag NRs/NWs synthesized.

\subsubsection{Surface Enhanced Raman Spectroscopy (SERS) and Microscopy}

We used a Raman microscope to obtain optical images of IDA electrodes or TED fabricated on $\mathrm{Si} / \mathrm{SiO}_{2}$. Optical images were obtained with an inverted Nikon Eclipse Ti microscope with a halogen lamp light source for sample illumination and a 40x aperture $(\mathrm{NA}=0.65)$. SERS was used to determine the enhancement factor of a Raman signal for molecules on Au/Ag NP chain/Au device. The intensity of the Raman signal was measured for 4-aminothiophenol (4-ATP) 
adsorbed on nanoparticle chains and on Au through Au-thiol bonding. The spectra were excited with $25 \mathrm{~mW}$ of $632.8 \mathrm{~nm}$ radiation from a HeNe laser using a Horiba micro Raman system in the $1000-1800 \mathrm{~cm}^{-1}$ range.

\subsubsection{Simultaneous Conductivity and SERS Mapping Measurements}

Raman spectral mapping was acquired at each and every pixel of the image and then interrogated to generate false color images on TED electrodes. The $\mathrm{Au} / \mathrm{Ag} \mathrm{NP}$ chain/Au TED was stepped from $-0.5 \mathrm{~V}$ to $-5 \mathrm{~V}$ and the SERS spectra were measured simultaneously. Simultaneous conductivity and SERS measurements were obtained in order to monitor resistive switching with SERS and understand the role of chain conductance on SERS. Figure 2.8 shows a schematic representation of the setup used for simultaneously obtaining conductivity and SERS measurements at $\mathrm{Au} / \mathrm{Ag}$ NP/Au devices coated with 4ATP.

\subsubsection{Dark Field Microscopy/Spectroscopy}

We used resonant Rayleigh dark-field scattering spectroscopy to obtain dark field images of IDA electrodes or TED fabricated on glass. Dark-field images and video were obtained with an inverted Nikon Eclipse Ti microscope with a halogen lamp light source and a dark-field condenser $(\mathrm{NA}=0.95-0.80)$ for sample illumination and a 100x variable aperture oil immersion objective ( $\mathrm{NA}=0.5-1.3)$ as shown in Figure 2.9. The detector used for measuring light scattering intensity as a function of wavelength was a multichannel air-cooled CCD camera used in a Horiba XPLORA-Inv confocal Raman system. 


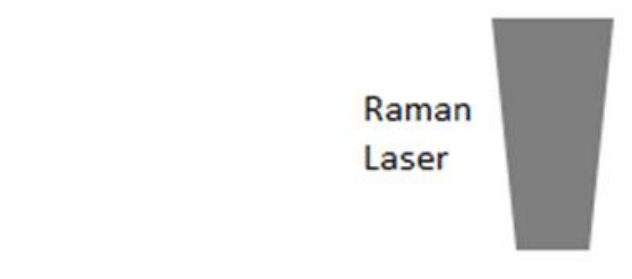

4-ATP Molecule

[4-Aminothiophenol]

Ag NPs

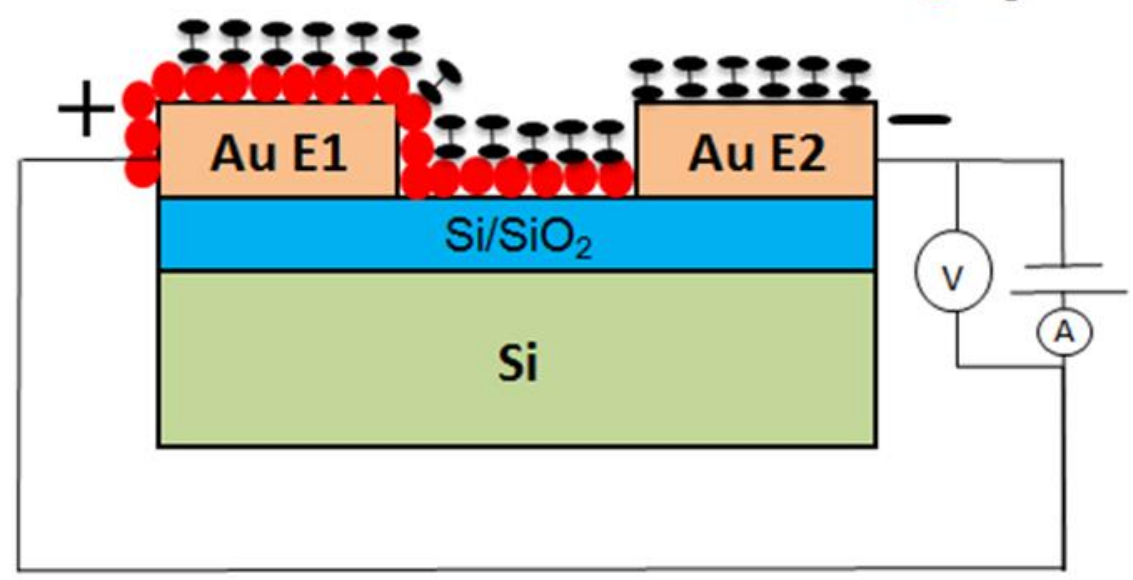

Figure 2.8. Instrumental set up for simultaneou Raman and electrical conductance measurement of Ag NP chain coated with 4-ATP on Au TED. 

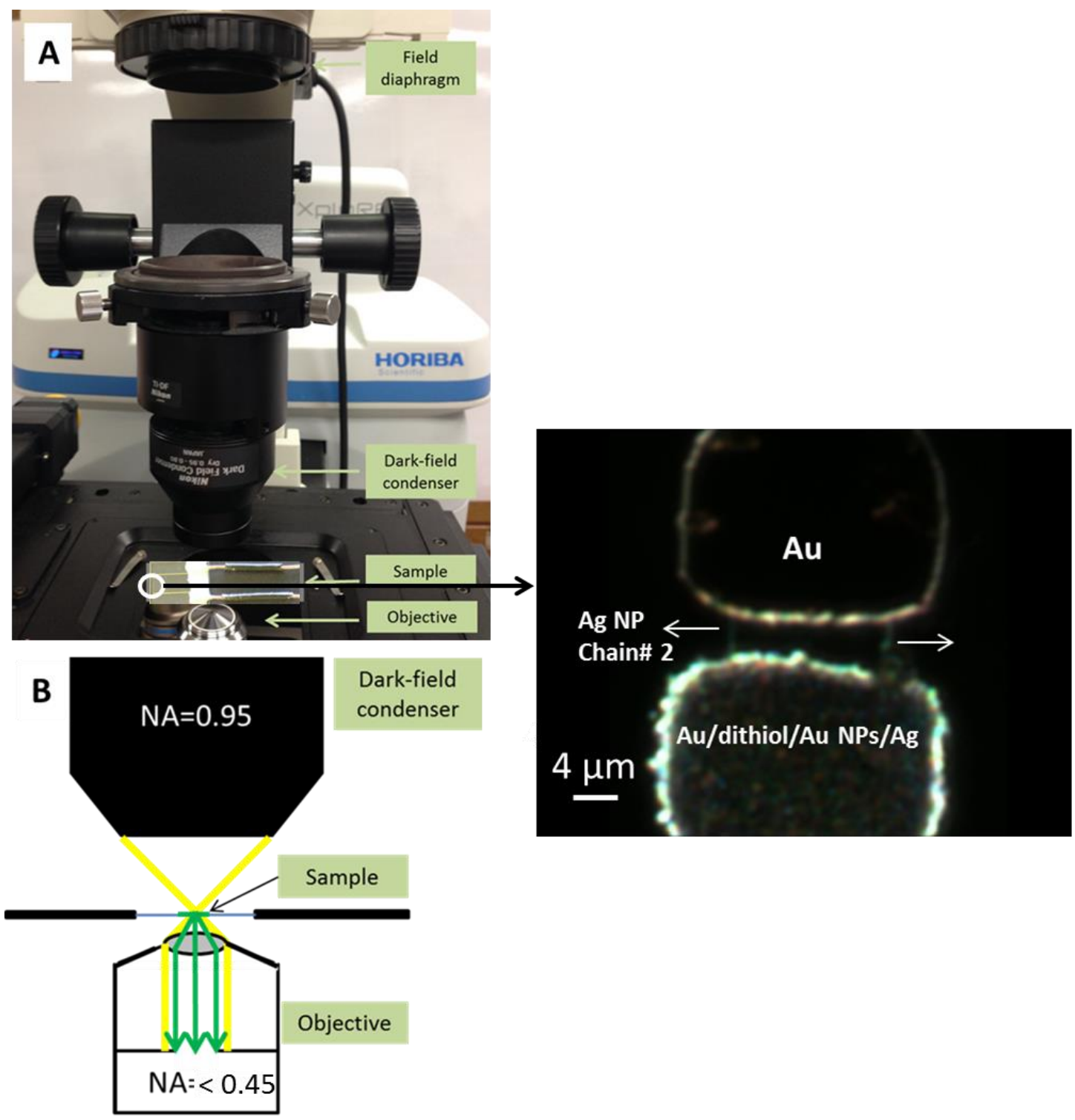

Figure 2.9. A) Apparatus for measuring dark-field microscopy and spectroscopy. B) Diagram showing the conical illumination. Yellow lines represent transmitted light missing the objective and green lines represent the scattered light detected by the objective. 


\subsection{Chemical and Electrochemical procedures}

\subsubsection{Electrodeposition of Ag on 5 Micron Gap Au Electrodes Using Cyclic Voltammetry}

Electrodeposition of Ag was performed on the differently prepared $\mathrm{Au}$ IDA/TED electrodes by connecting one side of the IDA/TED device as the working electrode and placing into a beaker with a solution containing $2.5 \times 10^{-4} \mathrm{M}$ $\mathrm{AgNO}_{3}$ and 0.1 M CTAB in $\mathrm{pH} 10.6$ phosphate buffer with an $\mathrm{Ag} / \mathrm{AgCl}$ reference electrode and platinum wire counter electrode to complete the three-electrode electrochemical cell. The deposition was performed in cyclic voltammetry mode by scanning the potential from $+400 \mathrm{mV}$ to $-200 \mathrm{mV}$ and then pausing at $-200 \mathrm{mV}$ for 1 hour as shown in the I-V plot in Figure 2.10. After 1 hour, the deposition was stopped and performed the same way on the other side of the IDA. Then the Au IDA device was removed from solution, rinsed thoroughly with nanopure water, and dried under a gentle flow of nitrogen. We performed stripping voltammetry to measure the charge of $\mathrm{Ag}$ on both sides of the IDA to quantify the amount of Ag, which was approximately $3.0 \pm 0.3 \times 10^{-5} \mathrm{C}$. We maintained a similar amount of charge when performing the deposition on the three differently treated IDAs. Ag deposition on Au/dithiol/Au NPs IDAs took one hour on each side. It took about 1.5 hours and 2 hours, respectively, for Ag deposition on $\mathrm{Au} /$ dithiol and bare $\mathrm{Au}$ IDAs to maintain the same amount of Ag. Ag deposition was performed for 1.5 hour for Au/dithiol/Au NPs devices deposited on only one side. 


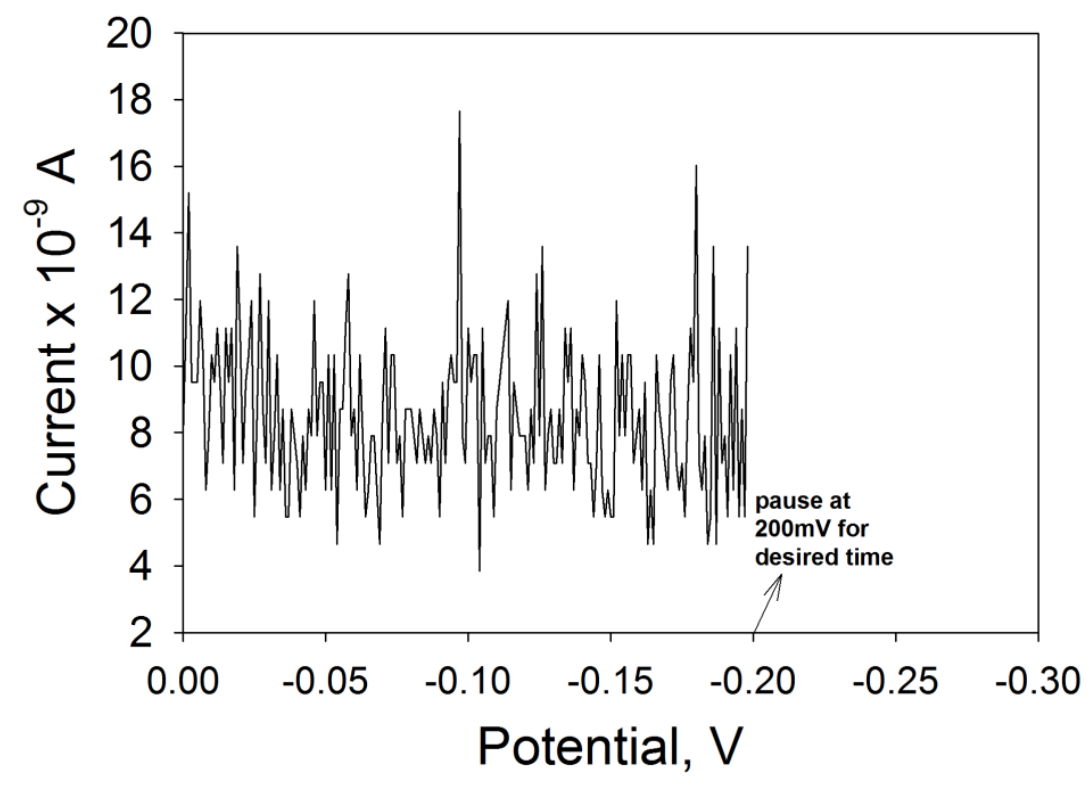

Figure 2.10. Linear Sweep Voltammogram with the reference electrode and counter electrode only in $2.5 \times 10^{-4} \mathrm{M} \mathrm{AgNO}_{3}$ in $0.1 \mathrm{M} \mathrm{CTAB}$ phosphate buffer solution. After pausing at potential at $-0.2 \mathrm{~V}$, the appropriate IDA device or TED was immersed in the solution and held for a desired amount of time at this potential for Ag deposition. It was also removed while still under potential control. 


\subsubsection{Electrodeposition of Ag on $5 \mu \mathrm{m}$ Au Electrodes Using Chronocoulometry}

Ag was also deposited directly onto each side or one side of an electrode device by chronocoulometry at $-0.3 \mathrm{~V}$ from a solution of $10 \mathrm{mM} \mathrm{AgNO}_{3}$ in water with no other supporting electrolyte until $6 \times 10^{-5} \mathrm{C}$ of charge (Q) passed as shown in the Q-t plot in Figure 2.11. This led to a compact film of Ag NPs deposited onto each electrode with no dendrites or nanowires. These devices were used to test the effect of $\mathrm{Ag}$ deposition without $\mathrm{CTAB}$ and for studies involving post treatment with $\mathrm{CTAB}$ and a negatively-charged surfactant, sodium dodecyl sulfate (SDS), or trisodium citrate. Post treatment involved soaking the Ag-coated Au electrodes in an aqueous $0.1 \mathrm{M}$ solution of the surfactant or citrate for $1 \mathrm{~h}$, removing, rinsing thoroughly with water, and drying under $\mathrm{N}_{2}$. Alternatively, the device was soaked in a $1.0 \times 10^{-4} \mathrm{M}$ solution of the surfactant or citrate for 5-10 min, removed, and allowed to air dry for a few hours without rinsing with water first. This results in a thin drop-cast film of the surfactant on the Ag-coated IDA device or TED. Figure 2.12 shows SEM images of the IDA devices with Ag deposited using cyclic voltammetry in the presence of CTAB and using chronocoulometry in the absence of CTAB. 


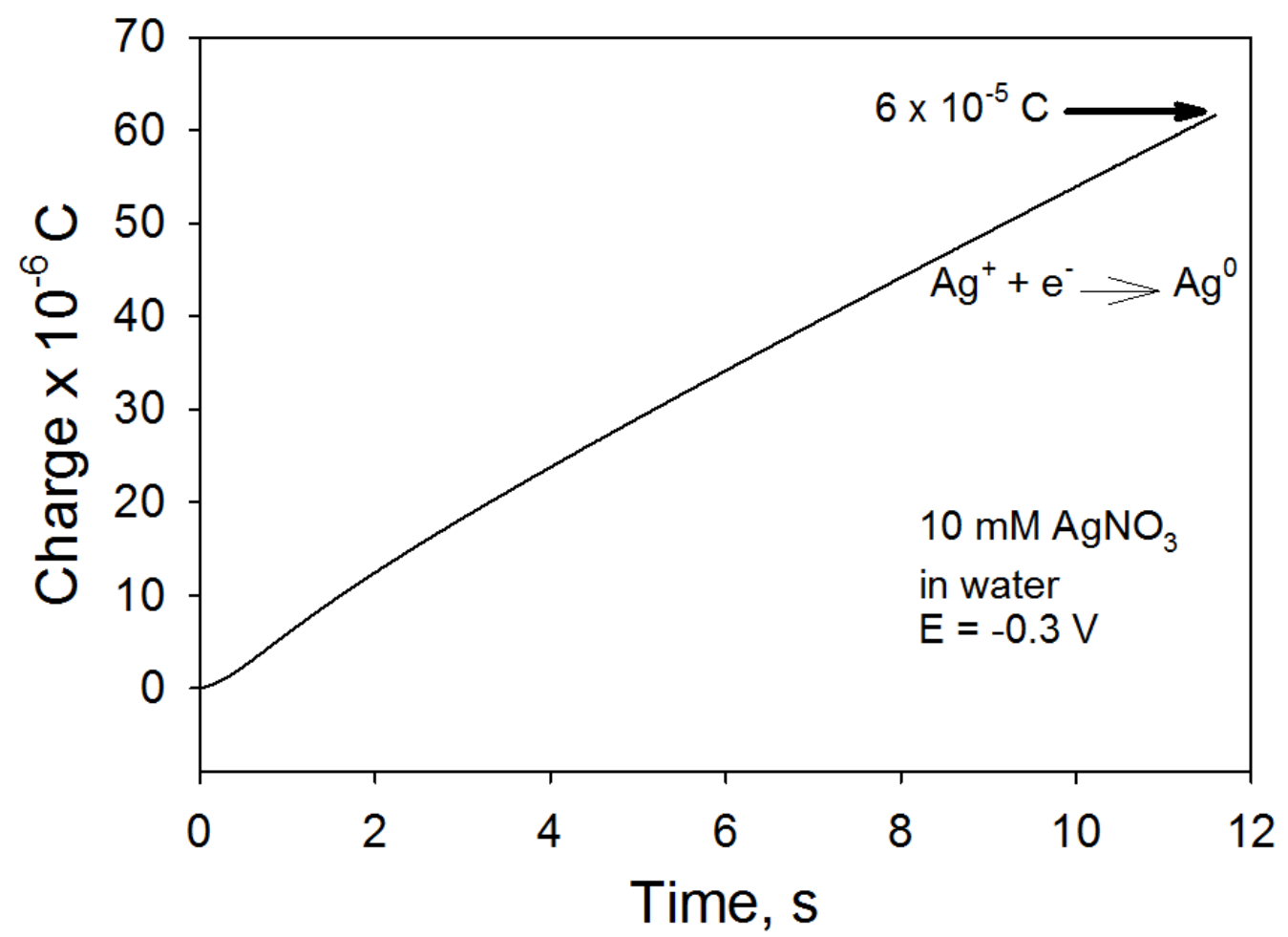

Figure 2.11. Chronocoulometric plot of IDA or TED electrode in $10 \mathrm{mM} \mathrm{AgNO3}$ in water at $-0.3 \mathrm{~V}$. 

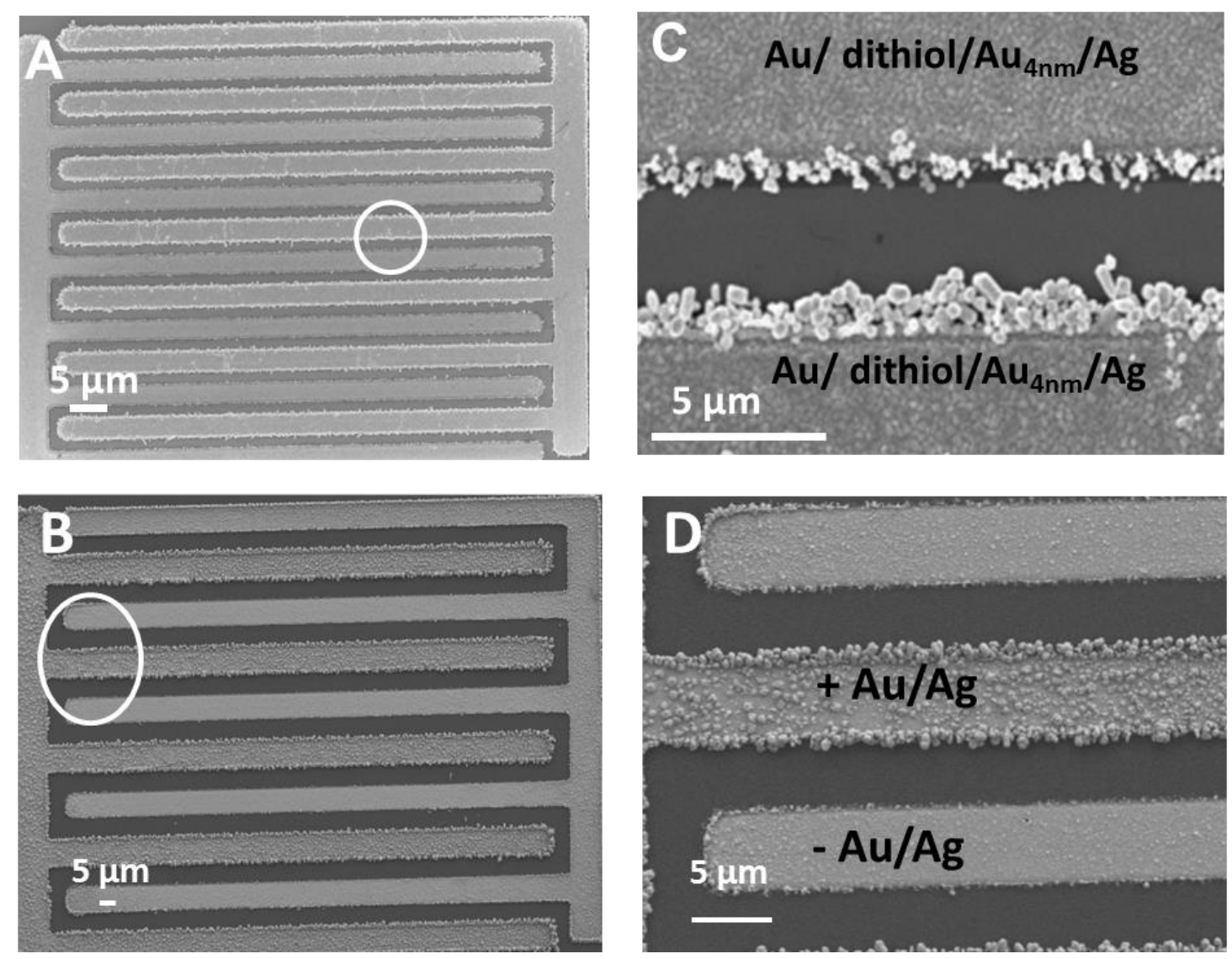

Figure 2.12. SEM images of IDA device with Ag deposited on each side. (A) $\mathrm{Au} /$ dithiol/Au NPs/Ag device with Ag deposited using cyclic voltammetry in the presence of CTAB and (B) Au/dithiol/Au NPs/Ag device with Ag deposited using chronocoulometry in the absence of CTAB. (C) and (D) are zoom in images of circled part shown in (A) and (B). 


\subsubsection{Ag NP Chain Formation}

After electrodeposition of Ag onto one or both electrodes of the Au IDA or $\mathrm{Au}$ TED, the current was measured as a function of voltage from $-1 \mathrm{~V}$ to $+1 \mathrm{~V}$ across the two electrodes using a two electrode set up. The current measured was always found to be at the pA level (except when post treated with a drop-cast film of surfactant or citrate), which confirmed that Ag did not electrodeposit directly across the two Au IDA electrodes and make a connection. The pA current level was the same for the Au IDA electrodes before Ag electrodeposition and similar to the two electrodes not hooked up to anything (the instrument noise). Later, the current was measured as a function of time for voltages ranging from 1 to $10 \mathrm{~V}$ up to $1000 \mathrm{~s}$ to trigger 1D Ag NP chain assembly across the IDA gap.

\subsubsection{Resistive Switching}

NP chain formation across $5 \mu \mathrm{m}$ electrode gaps resulted in switching behavior when $-5 \mathrm{~V}$ or $+5 \mathrm{~V}$ was applied to the device. The device could be made ON/OFF by applying negative or positive voltages. The nanoparticle chains were formed initially by using current-time experiments by holding the device at one potential for some period of time as described. After the device showed a rise in current, the potential across the gap was scan from $-5 \mathrm{~V}$ to $+5 \mathrm{~V}$ to observe the switching behavior in the I-V curve. Finally, we carried out endurance and switching speed studies by applying ON and OFF voltages at different pulse rate and cycles using chronoamperometry. 


\subsubsection{Electrodeposition of Phenol}

Electrodeposition was carried out on Au IDA electrodes that consisted of E1 and E2 electrodes. E1 and E2 represent the two sets of IDA fingers. Our procedure is similar to a previous method developed in our group for electropolymerization of phenol, except that we used 100 cycles instead of 20 cycles. ${ }^{38}$ Phenol was electropolymerized on E2 by cycling the potential (100 cycles) from 0 to $1.2 \mathrm{~V}$ at $100 \mathrm{mV} / \mathrm{s}$ in a $5 \mathrm{mM}$ phenol solution in $0.1 \mathrm{M} \mathrm{H}_{2} \mathrm{SO}_{4}$ solution with an $\mathrm{Ag} / \mathrm{AgCl}$ reference electrode and $\mathrm{Pt}$ counter electrode. Figure 2.13 shows the cyclic voltammogram of an Au IDA electrode immersed in $5 \mathrm{mM}$ phenol in $0.1 \mathrm{M} \mathrm{H}_{2} \mathrm{SO}_{4}$ solution. The voltammogram of phenol exhibited an irreversible oxidation peak at $1.1 \mathrm{~V}$. This indicates an irreversible oxidation reaction of the monomer on the electrode surface. During successive cycles, the peak current decreased. The fast decrease in the oxidation current is observed in the electropolymerization process with continuous potential cycling. This indicates that the growth of these polymer films is self-limiting due to the insulating nature of polyphenol.

\subsubsection{Electrodeposition of Ag NWs on Au IDA}

Ag NWs were grown on one set of electrodes (E1) by electrodeposition of $6.0 \times 10^{-5} \mathrm{C}$ of $\mathrm{Ag}(\mathrm{t} \sim 20 \mathrm{~s})$ from a solution containing $5 \mathrm{mM} \mathrm{AgNO}_{3}$ in aqueous $0.1 \mathrm{M} \mathrm{H}_{2} \mathrm{SO}_{4}$ at $-0.3 \mathrm{~V}$ using an $\mathrm{Ag}$ wire quasi reference electrode and Pt wire counter electrode using the procedure developed in our group. ${ }^{38}$ The deposition was performed in chronocoulometry mode by connecting one wire lead of the IDA 


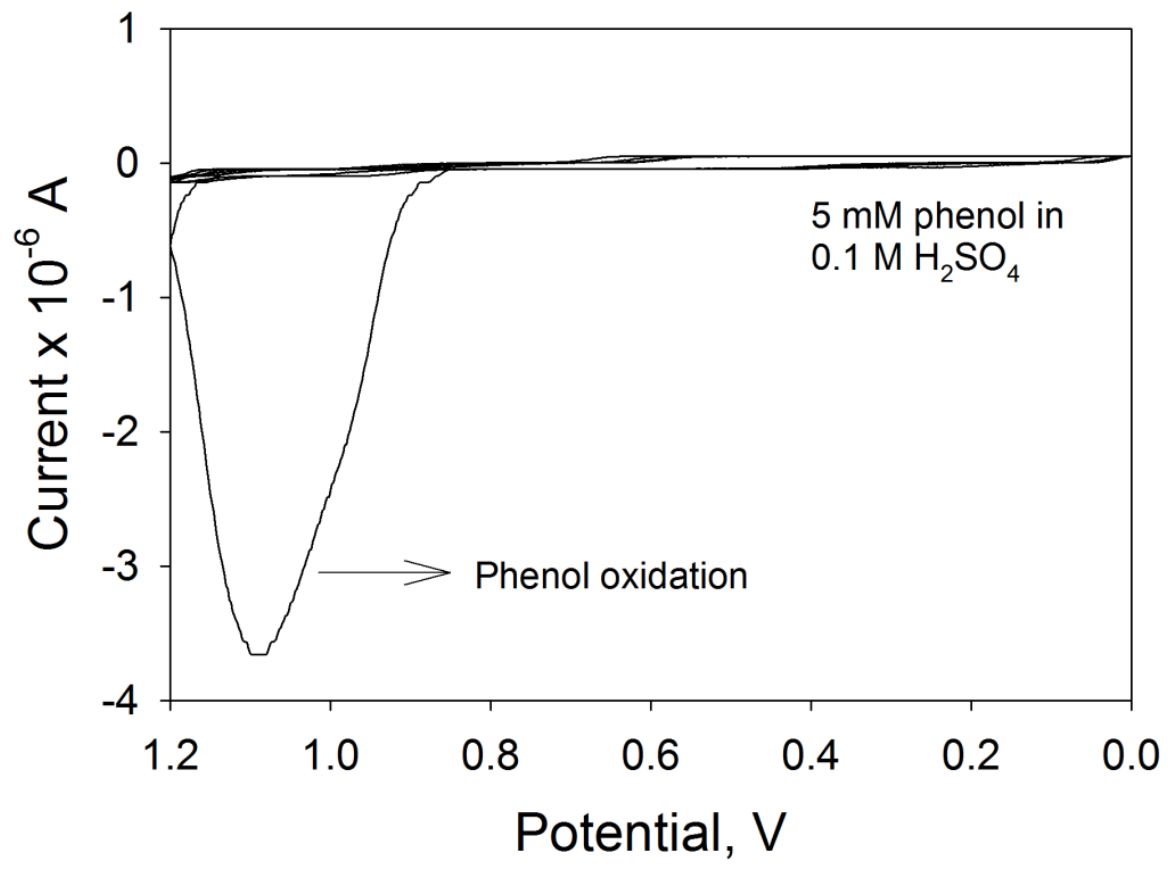

Figure 2.13. Cyclic voltammetry of one side of an Au IDA device in $5 \mathrm{mM}$ phenol in $0.1 \mathrm{M} \mathrm{H} 2 \mathrm{SO} 4$ for the first 10 cycles. Phenol oxidation at $1.1 \mathrm{~V}$ on the first cycle leads to a polyphenol film on the electrode that blocks phenol oxidation in subsequent scans. Electropolymerization of phenol on one set of Au IDA electrodes. I-V plot shows first 10 cycles 
to the potentiostat as the working electrode. Figure 2.14 shows the chronocoulometric plot of an Au IDA electrode in $5 \mathrm{mM} \mathrm{AgNO}_{3}$ at $-0.3 \mathrm{~V}$.

\subsubsection{Ag Electrodeposition on Glass/ITO}

The nonseeded glass/ITO/MPTMS and seeded glass/ITO/MPTMS/Au NP seed (4 nm) electrodes were used as two working electrodes along with an $\mathrm{Ag} / \mathrm{AgCl}$ reference electrode and a $\mathrm{Pt}$ wire counter electrode in an electrochemical cell with a deposition solution containing $2.5 \times 10^{-4} \mathrm{M} \mathrm{AgNO}_{3}$ and $0.1 \mathrm{M} \mathrm{CTAB}$ electrolyte. Figure 2.15 shows the setup. Our procedure is similar to the previous method developed in our group for synthesizing Au NRs electrochemically on glass/ITO electrode surfaces. ${ }^{29}$ Ag deposition was performed in cyclic voltammetry mode as follows. First, both the non-seeded and seeded electrodes were attached to the working electrode lead and held out of the solution while the reference and counter electrodes were placed into the deposition solution. Next, with the two working electrodes still out of the solution, the potential was scanned from $0.2 \mathrm{~V}$ to a final potential $\mathrm{x}$, where $\mathrm{x}$ was varied from $-100 \mathrm{mV}$ to $-500 \mathrm{mV}$. Once potential $\mathrm{x}$ was reached, it was held constant (paused) and the two working electrodes were lowered into the solution under potential control for an amount of time ranging from 30 to $120 \mathrm{~min}$. After the desired time, both working electrodes were then removed from the solution while the potential was still held constant at $\mathrm{x}$, rinsed thoroughly with nanopure water, and dried under $\mathrm{N}_{2}$ prior to SEM and stripping voltammetry characterization. Figure 2.16 shows the scheme for direct electrodeposition of Ag nanostructures on the ITO slides using a seed mediated 


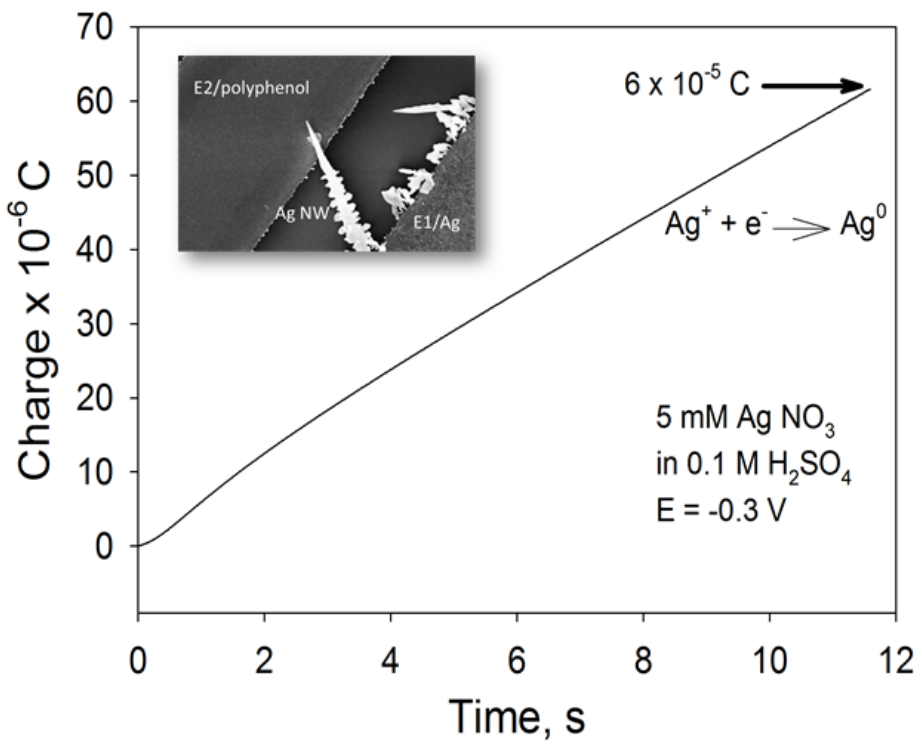

Figure 2.14. Ag deposition on Au IDA using chronocoulometry technique 


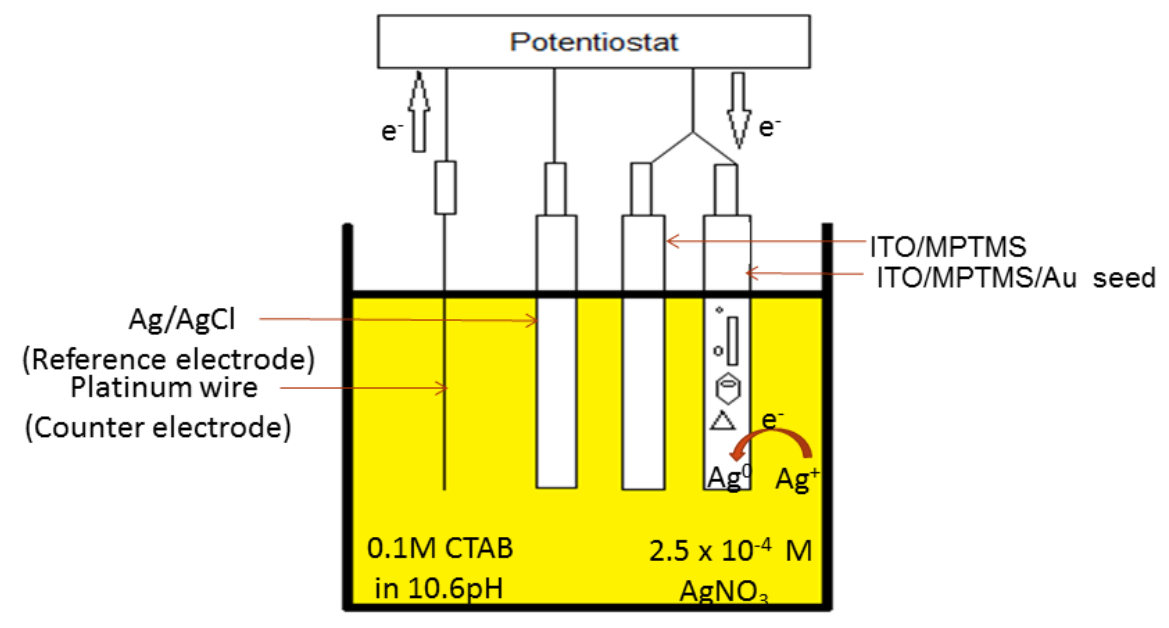

Figure 2.15. Set up for the Electrochemical Seed-Mediated Growth of Ag 


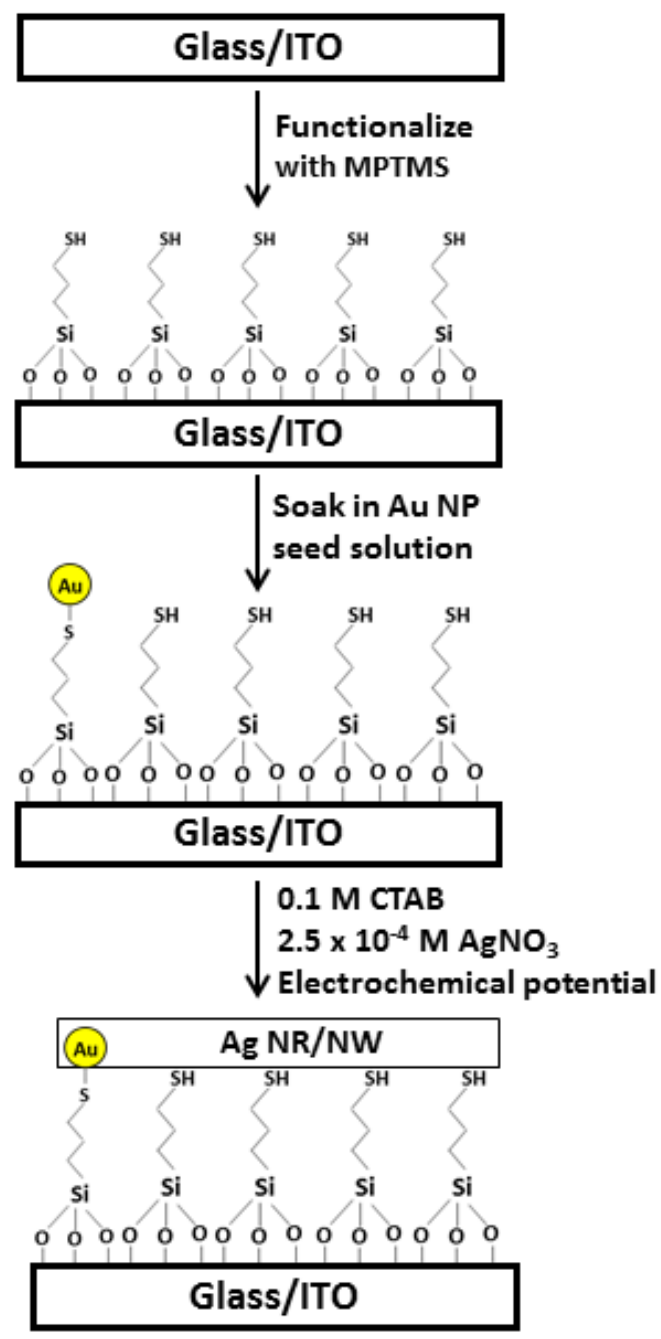

Figure 2.16 A schematic illustration of the growth of Ag nanostructures directly on the surfaces of glass/ITO by the seed-mediated growth procedure. 
growth procedure. The glass/ITO/MPTMS/Au NP seed $(4 \mathrm{~nm})$ slides were prepared as already discussed and Ag electrodeposition was then carried out.

\subsubsection{Ag Stripping on Glass/ITO}

Ag was removed from the ITO electrodes using linear sweep voltammetry. The potential was scanned from $0.0 \mathrm{~V}$ to $0.8 \mathrm{~V}$ in $0.1 \mathrm{M} \mathrm{H}_{2} \mathrm{SO}_{4}$ solution with an $\mathrm{Ag} / \mathrm{AgCl}$ reference electrode and $\mathrm{Pt}$ wire counter electrode at a scan rate of 0.01 $\mathrm{V} / \mathrm{s}$. The amount of Ag on the sample was determined by integrating the charge under the stripping peak.

\subsubsection{Humidity Control Technique}

Ag NP chain formation across the $5 \mu \mathrm{m}$ gap electrode is humidity dependent. IDA devices functionalized with dithiol and Au NP seeds were electrodeposited with $\mathrm{Ag}$ in the presence of $\mathrm{CTAB}$. After Ag deposition, we placed the device in a humidity chamber. The current was monitored with time at a constant potential from 0 to $10 \mathrm{~V}$ between the two electrodes while the sample was exposed to different levels of humidity. Different levels of humidity were controlled by bubbling nitrogen through water. The humidity was measured using a humidity monitor (AcuRite digital humidity and temperature monitor). 


\section{CHAPTER III}

\section{ELECTROCHEMICAL DEPOSITION OF AG NANOWIRES/NANORODS BY SEED-MEDIATED GROWTH DIRECTLY ON ELECTRODE SURFACES}

\subsection{Introduction}

It is well known that the optical, magnetic and electronic properties of metal NPs are highly dependent on the shape, size, and composition of metal NPs. The controllable synthesis of one dimensional nanostructures is one particular shape that has received great attention. In particular, NRs and NWs play an important role in fabricating nanoscale electronic, ${ }^{161,162}$ sensing, ${ }^{12,163}$ optoelectronic, ${ }^{164}$ and plasmonic devices. ${ }^{165}$ There are generally three methods for synthesizing and assembling 1D nanostructures on surfaces. The first method is by standard lithographic methods, where a material is etched or deposited into 1D nanostructures on a surface through a patterned resist. ${ }^{50}$ This method allows wellcontrolled size and shape of nanostructures and patterned assemblies, but the metals are amorphous. The second method involves solution or vapor phase synthesis of 1D nanostructures followed by dispersion in solution and subsequent assembling on surfaces. ${ }^{166}$ Usually there is some dispersity in shape and size and patterning is more difficult, but the nanostructures can be crystalline. The third method involves direct synthesis of 1D nanostructures from catalysts, seed particles, or templates that are attached directly to surfaces. ${ }^{27}$ The coverage and 
pattern depends on the catalyst and the materials can be crystalline, depending on the synthesis method.

Murphy and co-workers developed a seed-mediated growth solution method for synthesizing 1D NRs and NWs. ${ }^{159,167-169}$ Taub et al, later described the synthesis of Au NRs directly on surfaces from surface-bound Au nanoparticle seeds, ${ }^{25}$ followed by our group. ${ }^{26,27,30,95,170}$ Au NRs have also been synthesized by electrochemical methods. Our group synthesized Au NRs directly on surfaces electrochemically by the seed-mediated growth approach using electrochemical potential in place of a chemical reducing agent. ${ }^{29}$ This method does not require any templates to grow NRs, as the shape is promoted by the surfactant CTAB.

Here we compare the amount and the morphology of Ag nanostructures electrodeposited from a solution containing $2.5 \times 10^{-4} \mathrm{M} \mathrm{AgNO}_{3}$ and $0.1 \mathrm{M}$ cetyltrimethylammonium bromide (CTAB) onto non-seeded and $4 \mathrm{~nm} \mathrm{Au}-$ nanoparticle (Au NP) seeded mercaptopropyltrimethoxysilane (MPTMS)functionalized glass/indium tin oxide (glass/ITO) electrodes as a function of electrode potential and deposition time. There is a significantly larger amount of Ag deposited on the seeded electrode surface compared to the non-seeded electrode at lower potentials of $-0.15 \mathrm{~V}$ to $-0.30 \mathrm{~V}$ since the $\mathrm{Au} \mathrm{NP}$ seeds act as catalysts for Ag deposition; however, at more negative overpotentials of -0.40 to $0.60 \mathrm{~V}$, the amount of $\mathrm{Ag}$ deposited on both electrodes is approximately equal. In that case, the potential is negative enough that the rate of deposition is fast enough on glass/ITO that the Au seed catalyst does not make a difference. Ag nanorods 
(NRs) and nanowires (NWs) formed on the seeded surfaces, especially at more positive potentials, where deposition primarily occurred on the Au seed catalysts. Deposition of the Au seeds appeared as a separate peak in the voltammetry. This procedure mimics the seed-mediated growth observed in solution in the presence of CTAB. The yield, length, and aspect ratio of the Ag NRs/NWs depended on the deposition time; the average length ranges from $100 \mathrm{~nm}$ to $2 \mu \mathrm{m}$ for times of 30 to 120 min. One motivation is to electrochemically deposit 1D NWs and NRs across electrode gaps for nano electronics ad sensing applications.

\subsection{Experimental Section}

The cleaning procedure for glass ITO and functionalization with MPTMS and Au NP seeds. Refer to Chapter II section 2.2.5 and section 2.2.6.

The procedure for synthesis of Au seed NPs (NPs) is described in Chapter II Experimental section 2.1.2.

The Ag electrodeposition procedure is described in Chapter II section 2.4.7

The samples were characterized using SEM as outlined in Chapter II section 2.3.2.

Cyclic Voltammetry (CV). CV of glass/ITO/MPTMS and glass/ITO/MPTMS/Au NP seed were obtained in $0.1 \mathrm{M} \mathrm{CTAB}$ and $2.5 \times 10^{-4} \mathrm{M}$ $\mathrm{AgNO}_{3}$ solution using a $660 \mathrm{~A} \mathrm{CH}$ Instruments electrochemical workstation (Austin, $\mathrm{TX}$ ) with an $\mathrm{Ag} / \mathrm{AgCl}$ reference electrode and $\mathrm{Pt}$ wire counter electrode at a scan rate of $100 \mathrm{mV} / \mathrm{s}$. 


\subsection{Results and Discussion}

\subsubsection{Cyclic Voltammetry Studies}

Electrochemical deposition of Ag was performed in $\mathrm{pH} 10.6$ phosphate buffer solution in the presence of CTAB and $2.5 \times 10^{-4} \mathrm{M} \mathrm{AgNO}_{3}$ on $\mathrm{Au} \mathrm{NP}$ seeded and non-seeded glass/ITO. Au NP seeds were synthesized by reduction of Au salt with borohydride in the presence of citrate. ${ }^{21}$ The seed synthesis resulted in particles with 3 to $5 \mathrm{~nm}$ diameter. The Au NPs act as nucleation sites on the ITO electrode to catalyze the electrochemical growth of Ag nanostructures. Figure 3.1 shows CVs of glass/ITO, glass/ITO/MPTMS and glass/ITO/MPTMS/Au NP seed in $2.5 \times 10^{-4} \mathrm{M} \mathrm{Ag} \mathrm{NO}_{3}$ and $0.1 \mathrm{M} \mathrm{CTAB}$ solution. The most important observation from this experiment is that Ag deposition on seeded ITO starts approximately $-400 \mathrm{mV}$ to $-600 \mathrm{mV}$ earlier than on samples without seed and there is less cross-over in the CV on the reverse scan. This shift in potential is due to the presence of Au seeds which play the role of nucleation sites to catalyze Ag growth deposition. Based on this shift, the deposition potentials were chosen in this region to ensure that Ag deposition only occurs on the Au NP seeds.

The CV for glass ITO/MPTMS/Au NP seed has a clear peak at $-185 \mathrm{mV}$ corresponding to the Ag deposition of Au NP seeds. The glass/ITO electrode has a peak potential near $-980 \mathrm{mV}$, which is almost $800 \mathrm{mV}$ more negative. Also, on the reverse scan, the reduction current is larger at more positive potentials than on the forward scan. This indicates a large overpotential for initial nucleation of Ag 


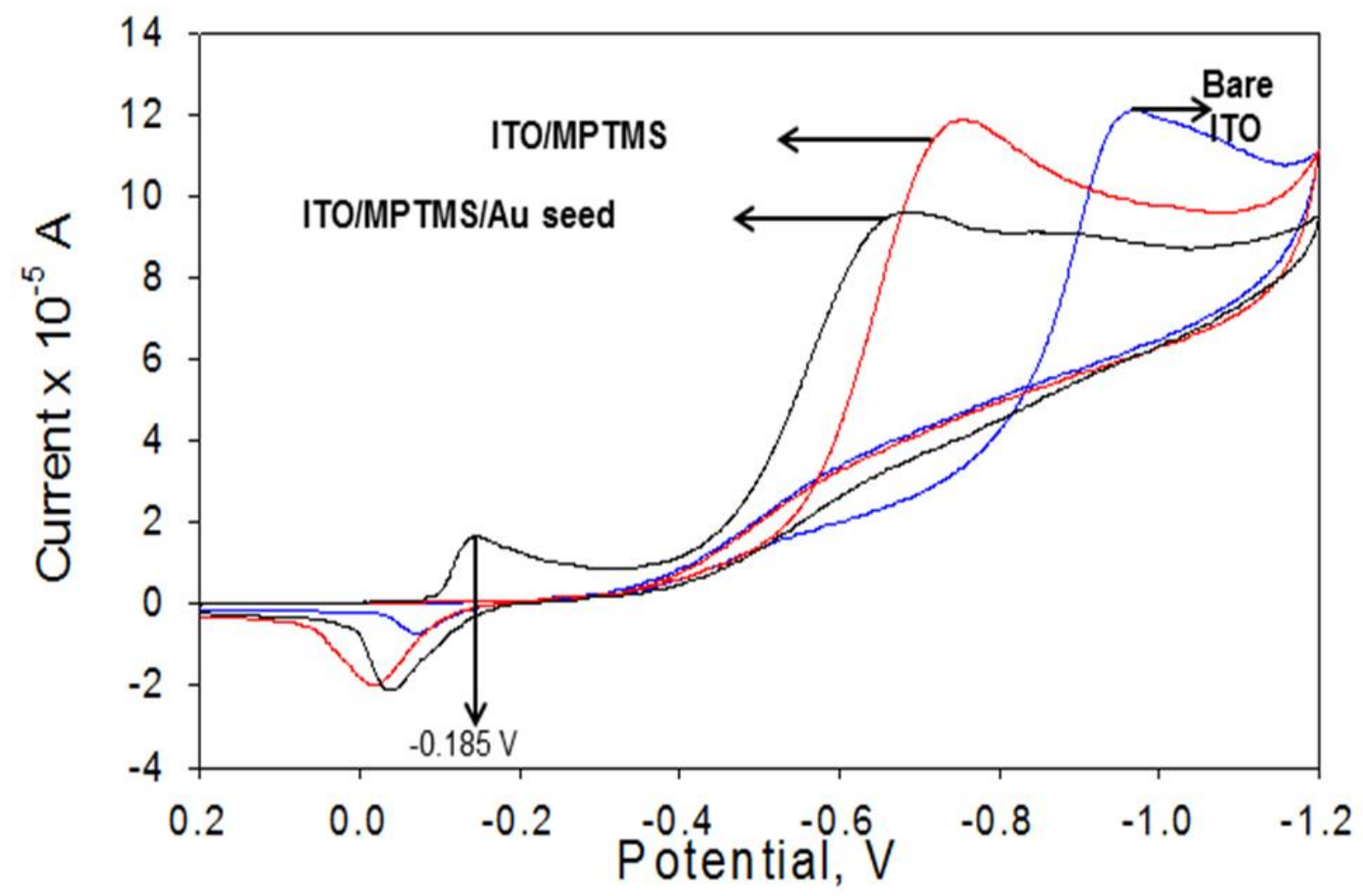

Figure 3.1. CVs of glass/ITO (blue), glass/ITO/MPTMS (red) and glass/ITO/MPTMS/Au NP seed (black) in $0.1 \mathrm{M} \mathrm{CTAB}$ plus $2.5 \times 10^{-4} \mathrm{M} \mathrm{AgNO}_{3}$ in $\mathrm{pH} 10.6$ buffer from $0.2 \mathrm{~V}$ to $-1.2 \mathrm{~V}$. 
deposits and then a smaller overpotential after some Ag had deposited. The glass/ITO/MPTMS has a lower overpotential with peak potential at $-780 \mathrm{mV}$, but similar higher reduction current on the reverse scan. It is not clear why MPTMS shifts the deposition potential positive, which is interesting.

\subsubsection{Stripping Voltammetry Studies}

We obtained stripping voltammetry data for all of the glass/ITO/MPTMS and glass/ITO/MPTMS/Au NP seed working electrodes after the Ag deposition procedure to determine the amount of Ag deposited on the surfaces as a function of electrode potential and time. Metallic Ag is stripped from the electrode using 0.1 $\mathrm{M} \mathrm{H}_{2} \mathrm{SO}_{4}$ solution as described in the Chapter II experimental section. Figure 3.2 shows the relation between stripping voltammograms and stripping voltammetry data of glass/ITO/MPTMS and glass/ITO/MPTMS/Au NP seeds. Figure $3.2 \mathrm{~A}$ shows the stripping voltammograms after depositing $\mathrm{Ag}$ at potential of $-0.2 \mathrm{~V}$ for 30 min. Glass/ITO/MPTMS black plot does not show any peak stripping peak of Ag compared to the sharp stripping peak of Ag for glass/ITO/MPTMS/Au NP seeds around $0.4 \mathrm{~V}$ (red plot). This clearly demonstrates seed-mediated growth of Ag. Figure $3.2 \mathrm{~B}$ shows the stripping data of glass ITO/MPTMS and glass ITO/MPTMS/Au NP seeds obtained after Ag deposition for $30 \mathrm{~min}$ at potentials ranging from $-100 \mathrm{mV}$ to $-500 \mathrm{mV}$. For the non-seeded glass/ITO/MPTMS as shown in the red line, there was significant Ag deposition at potentials of $-400 \mathrm{mV}$ and more negative. For the glass/ITO/MPTMS/Au NP seed electrode, significant 

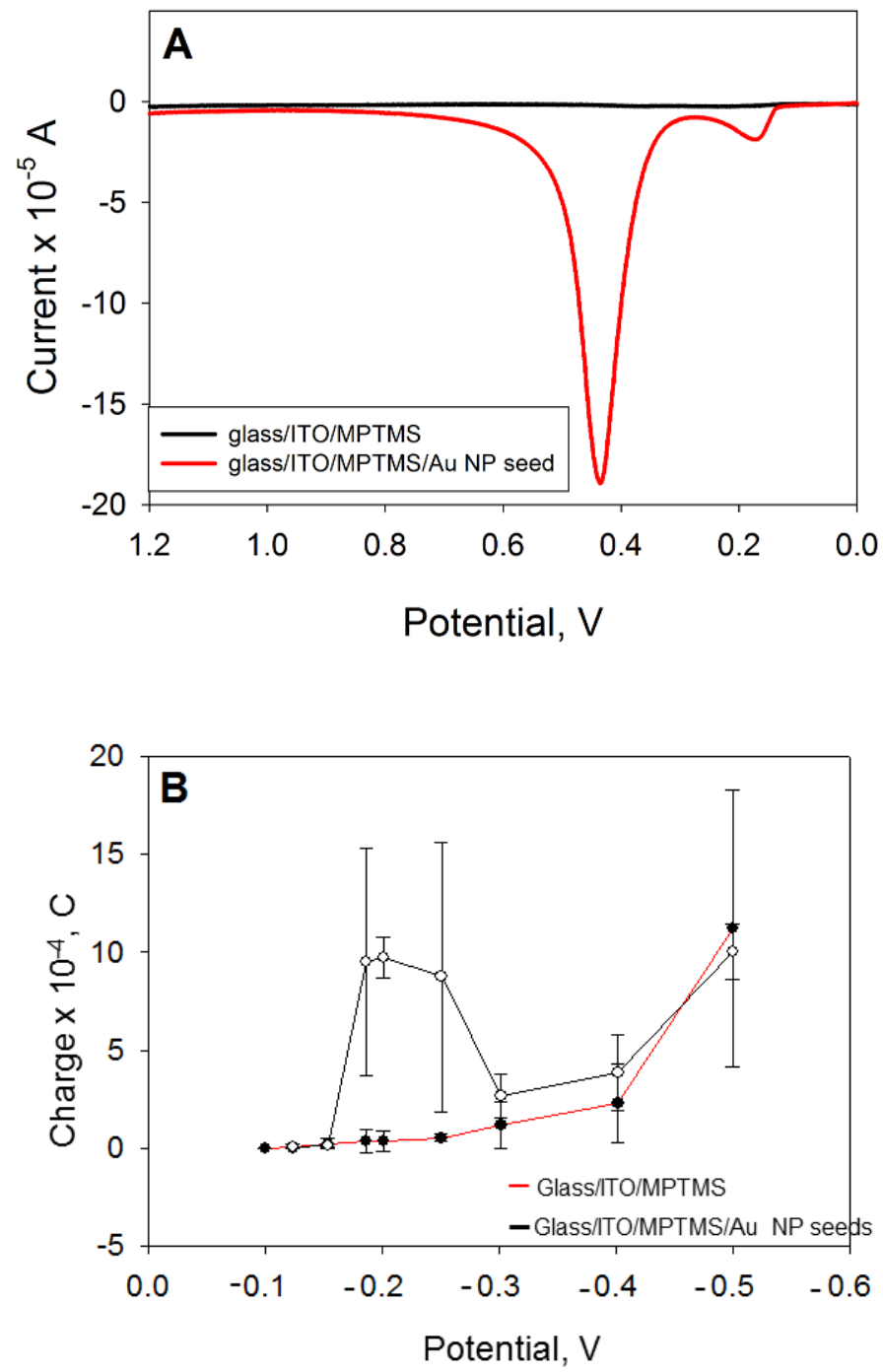

Figure 3.2. (A) Stripping Voltammogram for glass/ITO/MPTMS and glass/ITO/MPTMS/Au NP seeds after Ag deposition at $-0.2 \mathrm{~V}$ for 30 min. (B) Stripping voltammetry charge of glass/ITO/MPTMS (red) and glass/ITO/MPTMS/Au NP seed (black) electrodes after Ag deposition at different potentials for $30 \mathrm{~min}$ in $\mathrm{pH} 10.60 .1 \mathrm{M} \mathrm{CTAB}$ and $2.5 \times 10^{-4} \mathrm{M} \mathrm{AgNO}_{3}$. 
Ag deposited occurred at $\sim-150 \mathrm{mV}$, which is $250 \mathrm{mV}$ more positive of the nonseeded electrode. Significant deposition occurred over a wider range of potentials for the seeded surface defined at potentials of $(-185 \mathrm{mV}$ to $-250 \mathrm{mV})$, where the stripping of Ag was at a maximum, and then the amount of $\mathrm{Ag}$ decreased before increasing again at $-500 \mathrm{mV}$. It appears that deposition is somewhat hindered at more negative potentials, but we do not know the exact reason and this are large error bars. The amount of $\mathrm{Ag}$ is still larger on the seeded surfaces until $-500 \mathrm{mV}$, where the amount of $\mathrm{Ag}$ is about the same. Figure 3.3 shows the stripping voltammetry data of glass/ITO/MPTMS and glass/ITO/MPTMS/Au NP seed obtained after $\mathrm{Ag}$ deposition for $60 \mathrm{mins}$ at $-150 \mathrm{mV},-185 \mathrm{mV},-200 \mathrm{mV}$ and -250 $\mathrm{mV}$. We focused on the potentials from $-150 \mathrm{mV}$ to $-250 \mathrm{mV}$ for the time dependent studies because these potentials appeared to have the least amount of Ag deposited on the glass/ITO/MPTMS surface, indicating that primarily seedmediated growth occurred. It was observed that $\mathrm{Ag}$ deposition increased from $150 \mathrm{mV}$ to $-250 \mathrm{mV}$. There is not much difference in the Ag deposition for -200 $\mathrm{mV}$ and $-250 \mathrm{mV}$ as seen from the stripping charge, probably because the deposition was mass-transfer limited beyond $-200 \mathrm{mV}$. There is clearly almost no deposition on glass/ITO/MPTMS, indicating that seed-mediated growth occurred on the seeded surfaces.

Stripping experiments at longer time periods were carried out at potentials $150 \mathrm{mV},-185 \mathrm{mV}$ and $-200 \mathrm{mV}$. Figure 3.4 shows the stripping data after 


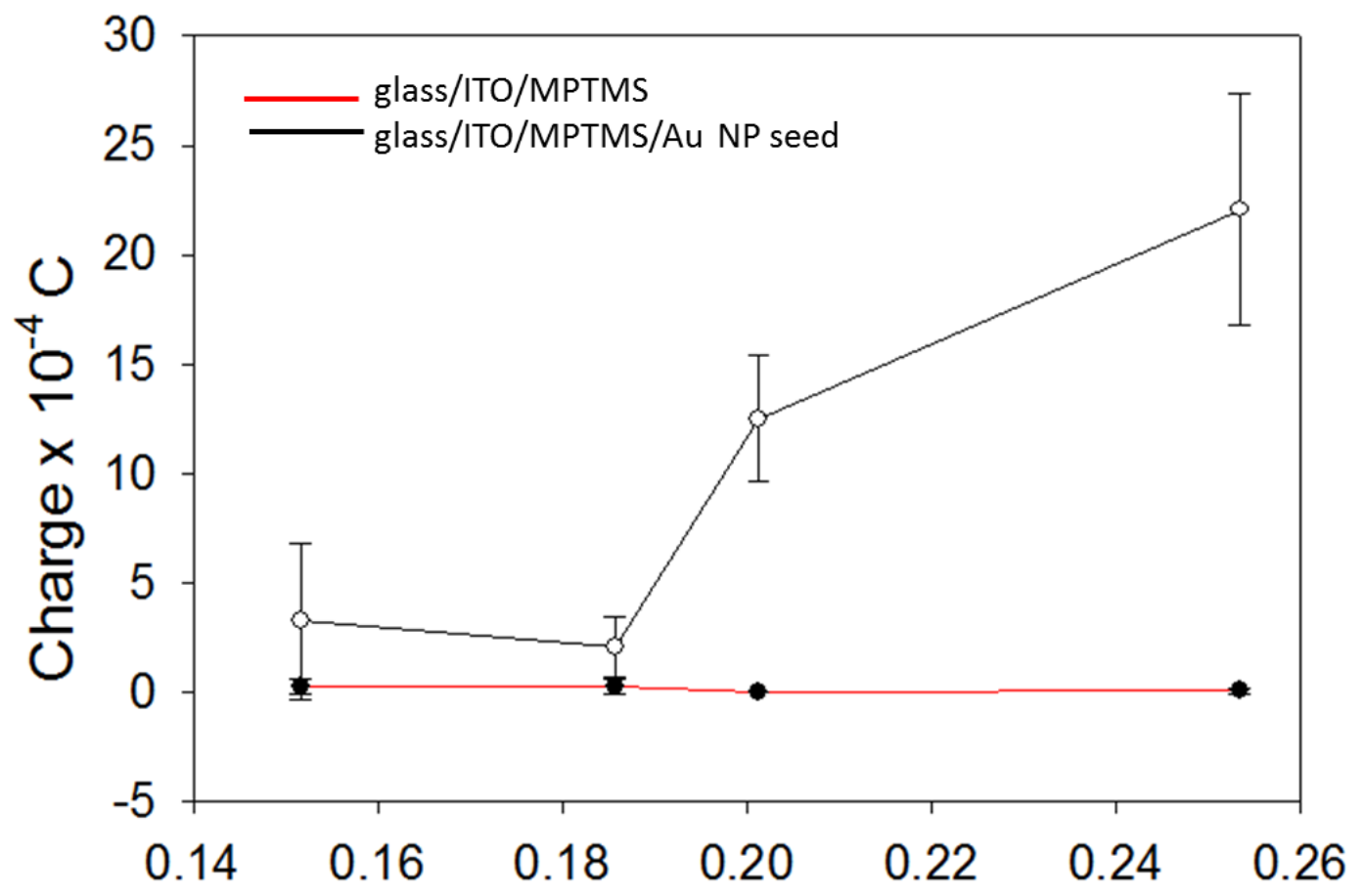

Figure 3.3. Stripping voltammetry of glass/ITO/MPTMS (red) and glass/ITO/MPTMS/Au NP seed (black) electrodes after Ag deposition at the different indicated potentials for $60 \mathrm{~min}$ in $0.1 \mathrm{M} \mathrm{CTAB}$ and $2.5 \times 10^{-4} \mathrm{M} \mathrm{AgNO}_{3}$ at $\mathrm{pH} 10.6$. 


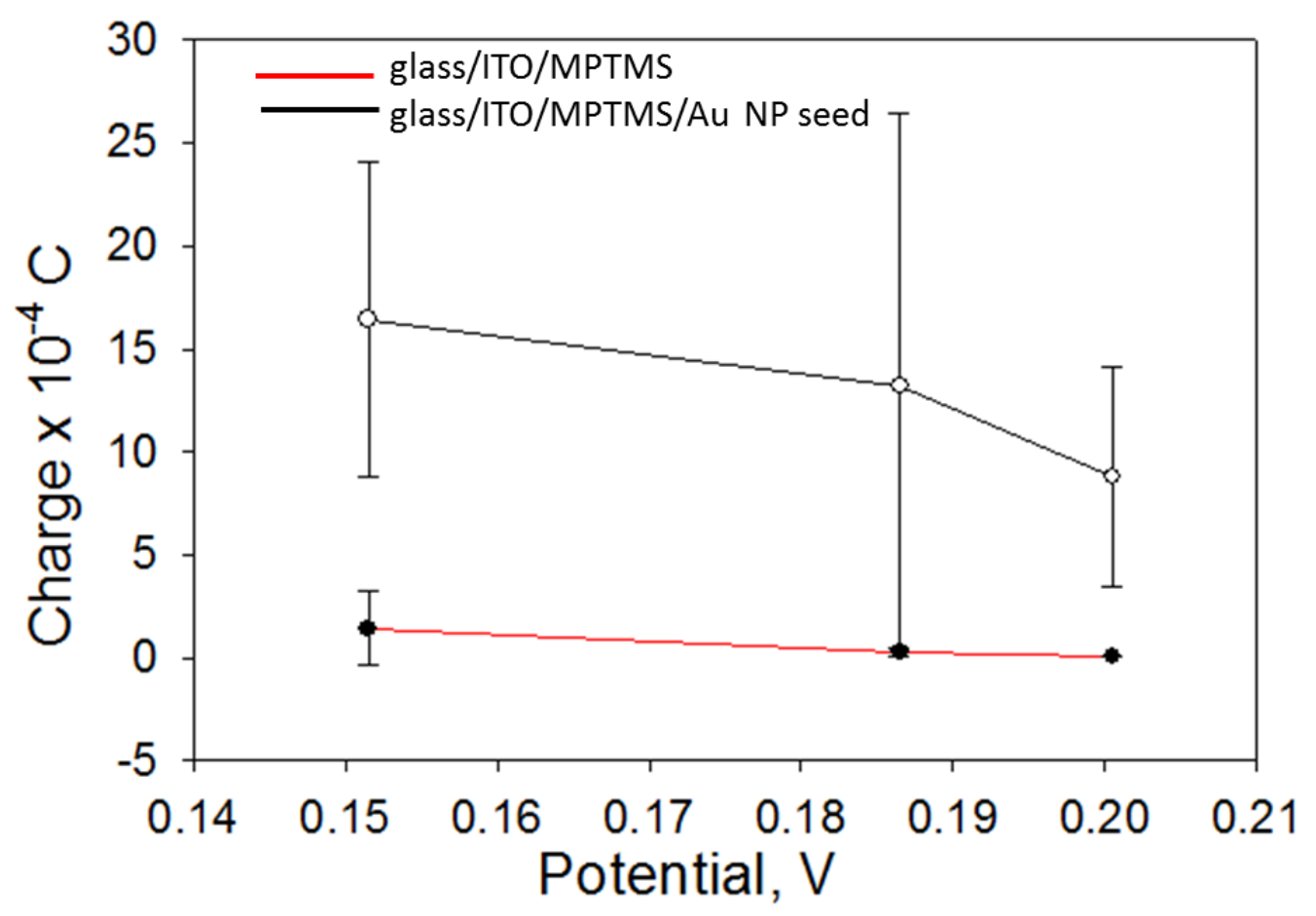

Figure 3.4. Stripping Voltammetry data of glass/ITO/MPTMS (red) and glass/ITO/MPTMS/Au NP seed (black) electrodes held at different potentials for $120 \mathrm{~min}$ in $0.1 \mathrm{M} \mathrm{CTAB}$ and $2.5 \times 10^{-4} \mathrm{M} \mathrm{AgNO}_{3}$. 
deposition for $120 \mathrm{~min}$ at these three potentials. We observed that as the deposition time increases, the amount of $\mathrm{Ag}$ increases and at $120 \mathrm{~min}$, the amount of $\mathrm{Ag}$ is almost equal at the three potentials. For seed-mediated growth, there should ideally be no Ag deposition in the absence of the Au NP seed. At these three potentials, the amount of Ag deposited increased with increasing deposition time. Figure 3.5 summarizes the stripping charge of $\mathrm{Ag}$ for potentials $-150 \mathrm{mV}$, $185 \mathrm{mV}$ and $-200 \mathrm{mV}$ at deposition times of 60 and $120 \mathrm{~min}$ by showing the stripping charge vs time plot. From this plot, it is further confirmed that no $\mathrm{Ag}$ deposition is seen on glass/ITO/MPTMS at these three potentials. On glass/ITO/MPTMS/Au NP seed, there is significant difference between deposition times of $60 \mathrm{~min}$ and $120 \mathrm{~min}$ and with large error bars.

\subsubsection{Microscopy Studies}

We obtained SEM images of the various glass/ITO/MPTMS and glass/ITO/MPTMS/Au NP seed electrodes at different potentials and at different time. Figure 3.6 shows SEM images of the glass/ITO/MPTMS at different potentials and at different time. There was minimal Ag deposition on the glass/ITO/MPTMS electrodes from 0 to $-250 \mathrm{mV}$ as expected based on the stripping data. The Ag deposited in the form of branched flower like structures and more $\mathrm{Ag}$ deposited on these non-seeded electrodes at potentials from $-300 \mathrm{mV}$ to $-500 \mathrm{mV}$. Figure 3.7 shows glass/ITO/MPTMS and glass/ITO/MPTMS/Au seed electrodes after deposition of $\mathrm{Ag}$ at $-150 \mathrm{mV}$ for 30,60 , and $120 \mathrm{~min}$. At this potential, there were fewer Ag deposits on the glass/ITO/MPTMS electrodes, 


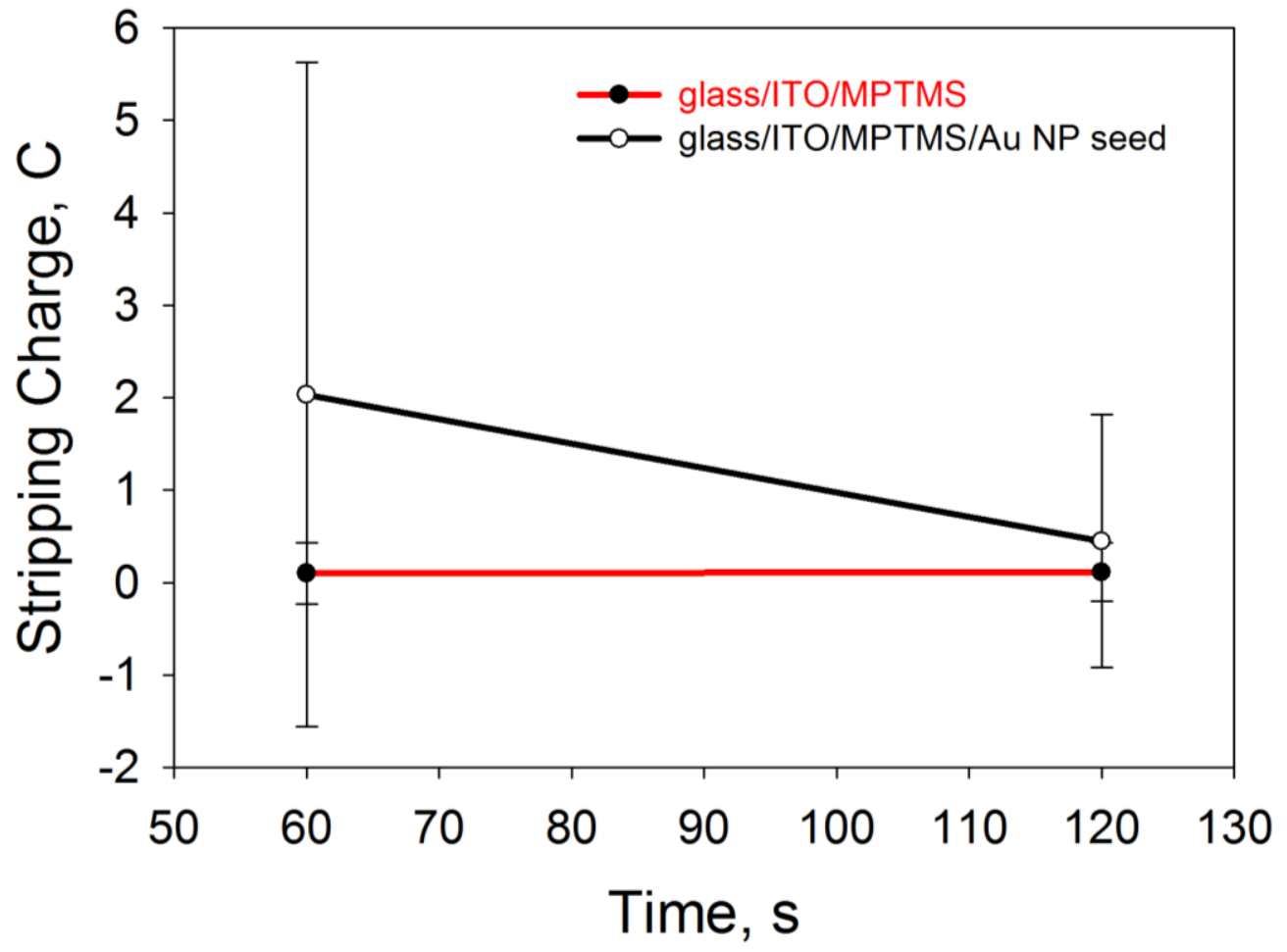

Figure 3.5. Stripping charge vs Ag deposition time plot for glass/ITO/MPTMS (red) and glass/ITO/MPTMS/Au NP seed (black) for potentials -150, -185 and $-200 \mathrm{mV}$ at 60 and $120 \mathrm{~min}$. 

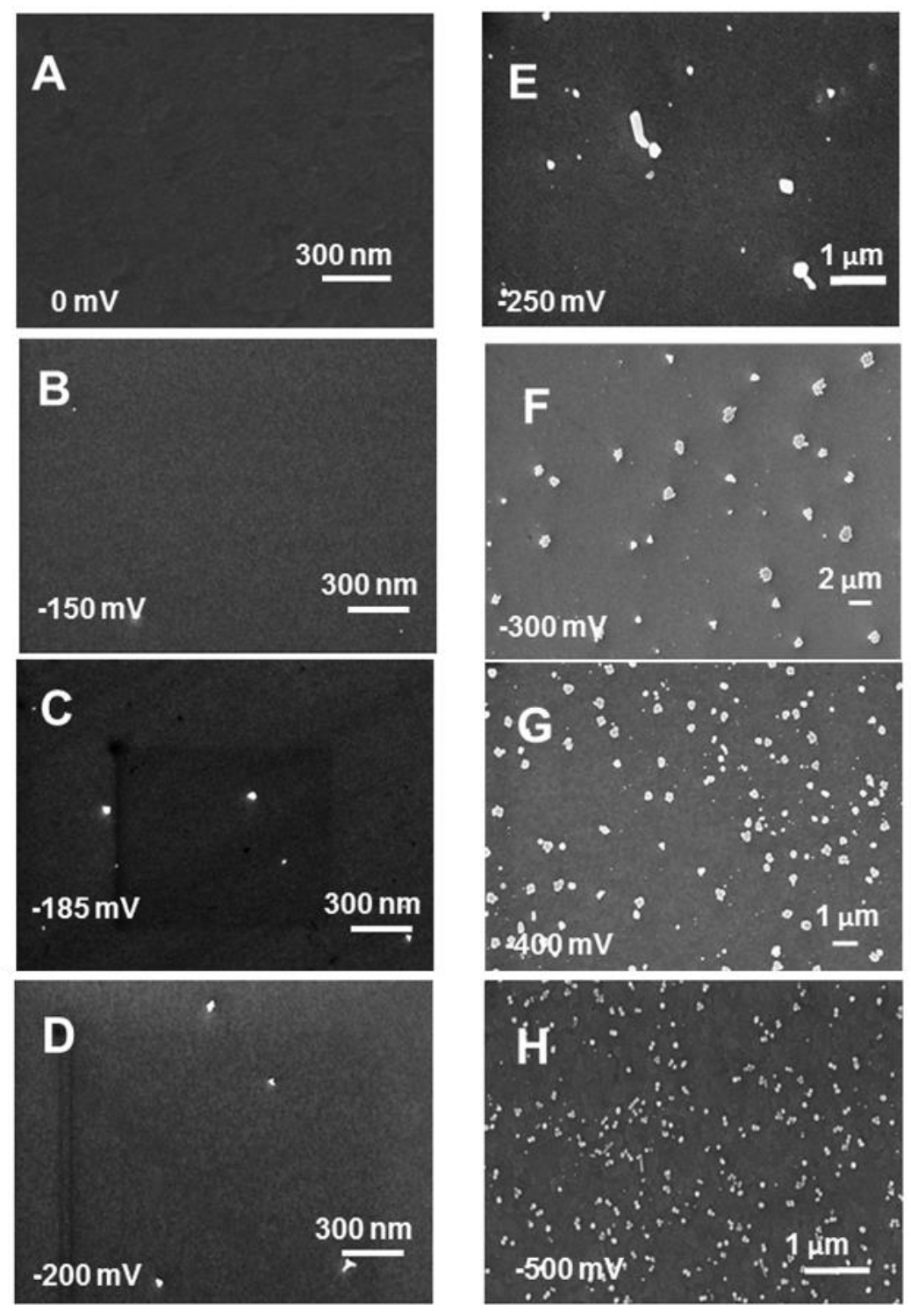

Figure 3.6. SEM images of glass/ITO/MPTMS at the indicated potentials for a deposition time of $30 \mathrm{~min}$. 


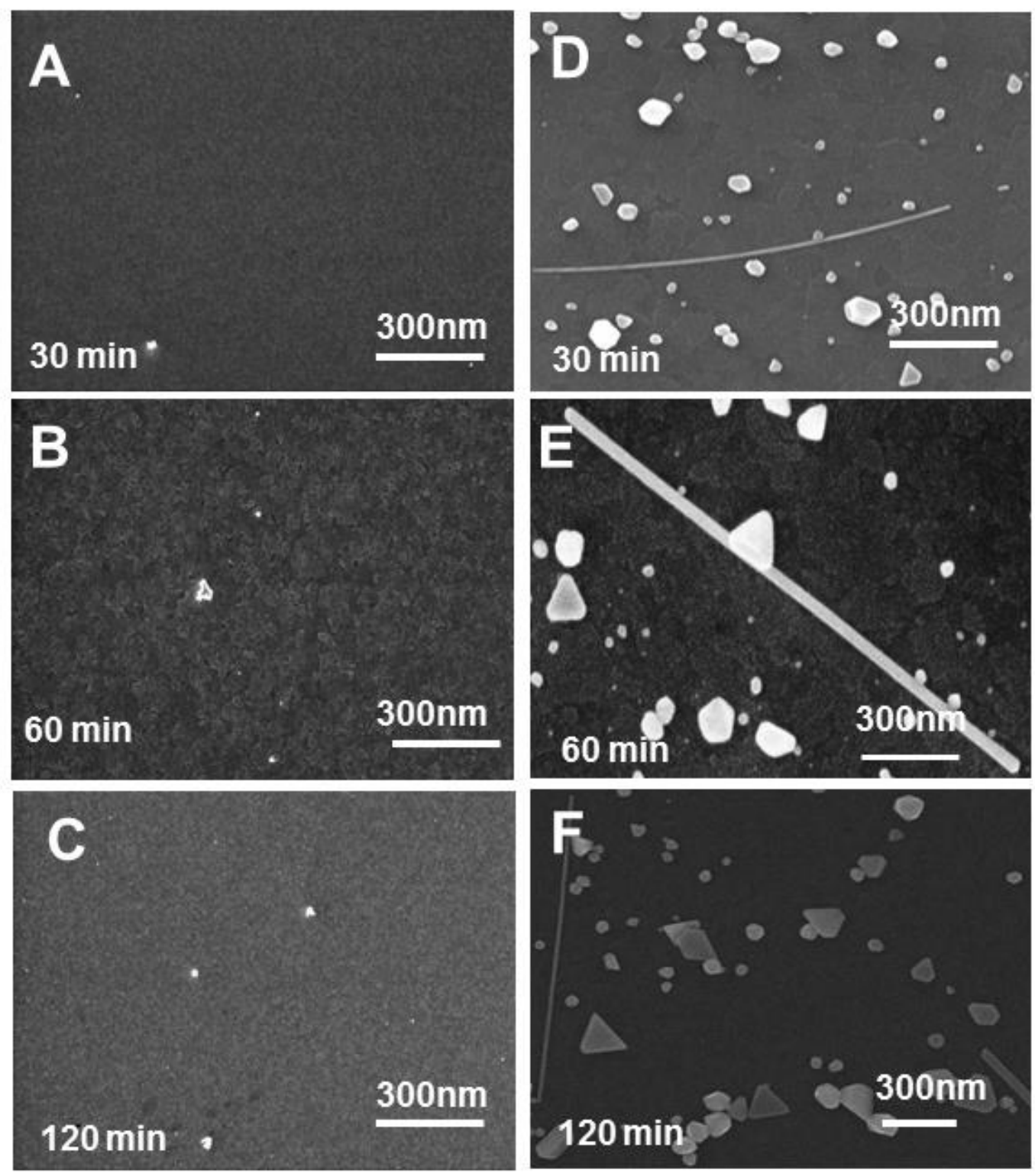

Figure 3.7. SEM images of glass/ITO/MPTMS (A, B, C) and glass/ITO/MPTMS/Au NP seed electrodes (D, E, F) held at $-150 \mathrm{mV}$ for 30, 60 and $120 \mathrm{~min}$. 
consistent with the stripping data, and there were a significant number of Ag NRs and NWs on the glass/ITO/MPTMS/Au NP seed electrodes along with spheres and plate-shaped particles. The NR yield ranged from 5 to $10 \%$. Figure 3.8 shows glass/ITO/MPTMS and glass/ITO/MPTMS/Au seed electrodes after the deposition of $\mathrm{Ag}$ at $-185 \mathrm{mV}$ for 30, 60, and $120 \mathrm{~min}$. Again, no significant amount of $\mathrm{Ag}$ deposited on the glass/ITO/MPTMS at this potential and a significant percentage of NRs/NWs formed on the glass/ITO/MPTMS/Au NP seed electrodes. The NR/NW yielded ranged from 5 to $15 \%$.

Figure 3.9 shows glass/ITO/MPTMS and glass/ITO/MPTMS/Au NP seed electrodes after deposition of $\mathrm{Ag}$ at $-200 \mathrm{mV}$ for 30, 60, and $120 \mathrm{~min}$. No deposition occurred on the electrode without Au seeds and Ag NRs/NWs formed on the Au NP-seeded electrode. The yield ranged from 5-15\%. When comparing the morphology of the Ag deposited at $-150,-185$ and $-200 \mathrm{mV}$, it is clear that the electrode potential has an effect on the shape of the Ag nanostructures. The reduction potentials of $-150 \mathrm{mV}$ to $-200 \mathrm{mV}$ favored the growth of $\mathrm{Ag} \mathrm{NRs} / \mathrm{NWs}$. The dimensions of NRs varied within the $-150 \mathrm{mV}$ to $-200 \mathrm{mV}$ as a function of potential and growth time. Table 3.1 compares the average length, aspect ratio, and yield of Ag NRs/NWs deposited on glass/ITO/MPTMS/Au NP seed electrodes at $-150,-185$ and $-200 \mathrm{mV}$ for 30,60 and $120 \mathrm{~min}$. In general, the average length of the NRs/NWs increased from 30 to $60 \mathrm{~min}$ at all potentials. 


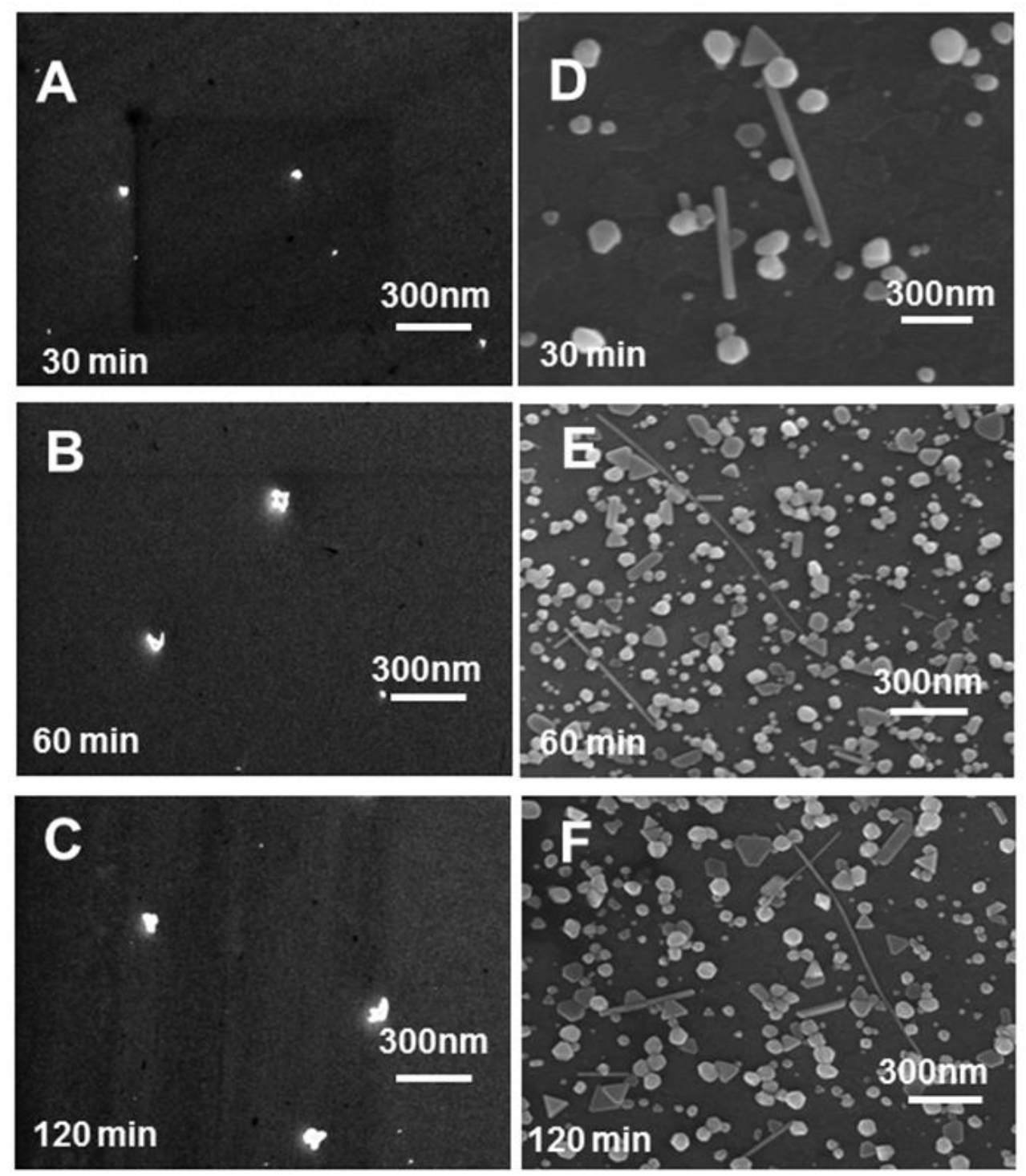

Figure 3.8. SEM images of glass/ITO/MPTMS (A, B, C) and glass/ITO/MPTMS/Au NP seed electrodes (D, E, F) held at $-185 \mathrm{mV}$ for 30, 60 and $120 \mathrm{~min}$. 

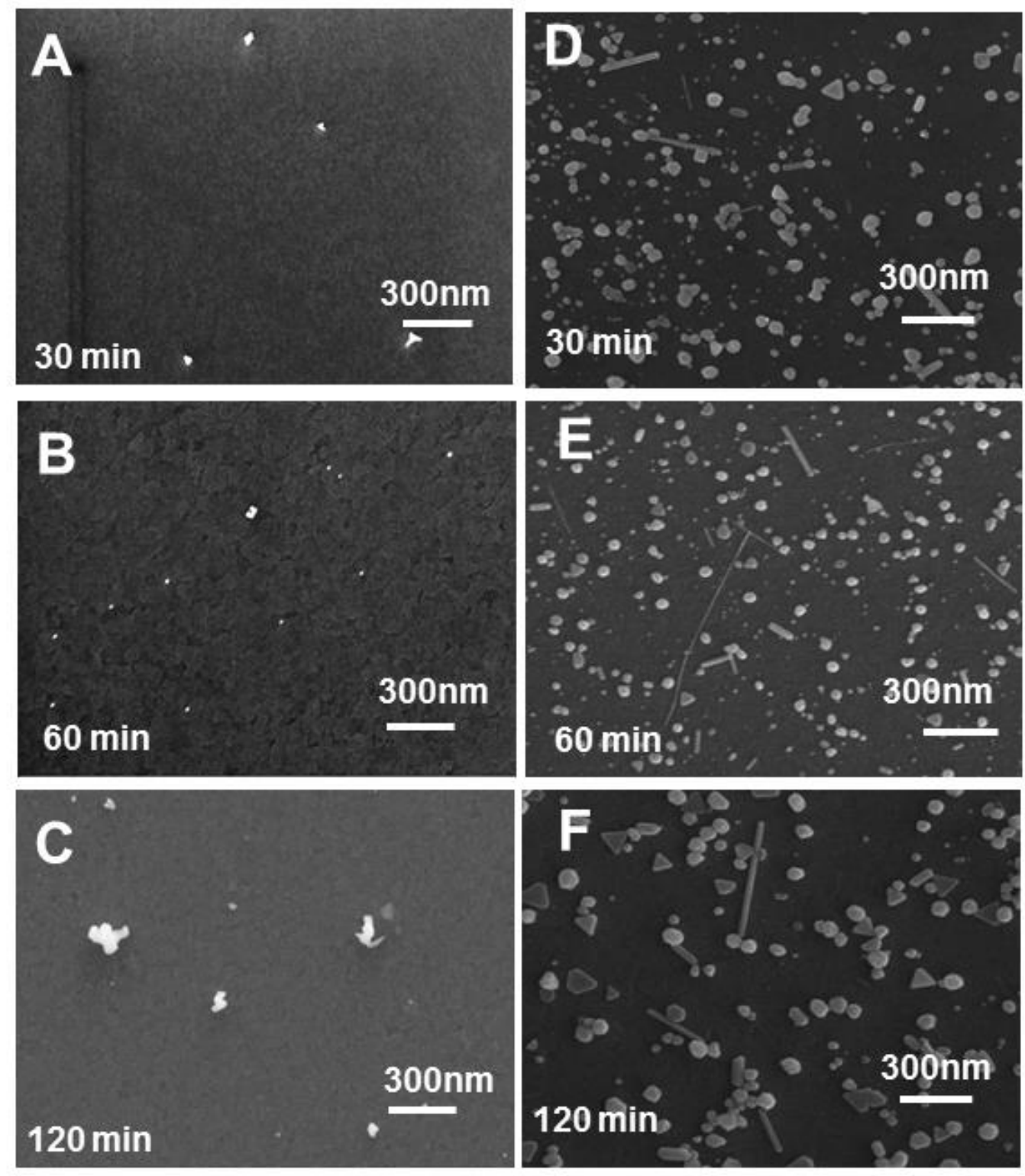

Figure 3.9. SEM images of glass/ITO/MPTMS (A, B, C) and glass/ITO/MPTMS/Au NP seed electrodes (D, E, F) held at -200 mV for 30, 60 and $120 \mathrm{~min}$. 
Table 3.1 Statistical data on the nanorods/nanowires grown by electrochemical seed-mediated growth as a function of potential and time.

\begin{tabular}{|c|c|c|c|}
\hline Potential (mV) & \multicolumn{3}{|c|}{-0.15} \\
\hline Time (min) & 30 & 60 & 120 \\
\hline Yield \% & $2-5 \%$ & $2-5 \%$ & $10-12 \%$ \\
\hline Aspect ratio & $35( \pm 23)$ & $57( \pm 41)$ & $33( \pm 19)$ \\
\hline $\begin{array}{l}\text { Coverage } \\
\left(\text { Particles } / \mu \mathrm{m}^{2}\right)\end{array}$ & $25( \pm 12)$ & $18( \pm 13)$ & $21( \pm 7)$ \\
\hline Rod length (nm) & $675( \pm 403)$ & $2611( \pm 1897)$ & $657( \pm 366)$ \\
\hline Potential (mV) & \multicolumn{3}{|c|}{-0.185} \\
\hline Time (min) & 30 & 60 & 120 \\
\hline Yield \% & $2-5 \%$ & $5-10 \%$ & $15-18 \%$ \\
\hline Aspect ratio & $11( \pm 6)$ & $45( \pm 33)$ & $35( \pm 18)$ \\
\hline $\begin{array}{l}\text { Coverage } \\
\left(\text { Particles } / \mu \mathrm{m}^{2}\right)\end{array}$ & $16( \pm 7)$ & $89( \pm 41)$ & $76( \pm 31)$ \\
\hline Rod length (nm) & $449( \pm 187)$ & $643( \pm 472)$ & $928( \pm 391)$ \\
\hline
\end{tabular}

\begin{tabular}{|c|c|c|c|}
\hline Potential (mV) & \multicolumn{3}{|c|}{-0.2} \\
\hline Time (min) & 30 & 60 & 120 \\
\hline Yield \% & $2-5 \%$ & $5-10 \%$ & $12-15 \%$ \\
\hline Aspect ratio & $11( \pm 4)$ & $41( \pm 25)$ & $11( \pm 6)$ \\
\hline $\begin{array}{l}\text { Coverage } \\
\left(\text { Particles } / \mu \mathrm{m}^{2}\right)\end{array}$ & $68( \pm 11)$ & $52( \pm 13)$ & $51( \pm 20)$ \\
\hline Rod length (nm) & $314( \pm 100)$ & $525( \pm 302)$ & $364( \pm 150)$ \\
\hline
\end{tabular}


The density of nanostructures (particle, rods, and other shapes) on the surface was larger for $120 \mathrm{~min}$ for $-185 \mathrm{mV}$ and $-200 \mathrm{mV}$. This could be due to high density of initial seed NPs or new self-nucleation sites formed. We found that the $-150 \mathrm{mV}$ appears to be the most favorable potential to grow longer NRs/NWs. Compared to with deposition times of 30 and $60 \mathrm{~min}$, the length increased from $\mathrm{nm}$ to a few microns. For $120 \mathrm{~min}$, the length of the NR did not increase further. The density of nanostructures on the surface ranged from 5 to 40 per $\mu \mathrm{m}^{2}$ for 30,60 and 120 min depositions at $-150 \mathrm{mV}$. I think it varies due to irreproducibility, not a real phenomenon. Further we tried this procedure to grow Ag NWs/NRs across Au and ITO gap electrodes, but did not observe any Ag NR/NW across the gap.

\subsection{Conclusions}

The morphology of Ag deposited on glass/ITO/MPTMS electrodes in CTAB depends on the presence or absence of Au NP seeds on the surface, the electrode potential, and the deposition time. For non-seeded glass/ITO/MPTMS electrodes, $\mathrm{Ag}$ is deposited in the form of branched or snow flake structures at potentials ranging from -400 to $-500 \mathrm{mV}$. At $0.0 \mathrm{~V}$ to $-300 \mathrm{mV}$, there is very little $\mathrm{Ag}$ deposition on the non-seeded surface but sporadic regions with some Ag on the electrode exist. On the glass/ITO/MPTMS/Au seed electrodes, Ag deposits from $150 \mathrm{mV}$ to $-500 \mathrm{mV}$ and the amount of $\mathrm{Ag}$ deposited is greater than that on the nonseeded surfaces at all potentials. From $-150 \mathrm{mV}$ to $-250 \mathrm{mV}$, the seed-mediated growth is optimal because there is little or no Ag deposition on nonseeded surfaces. Also from $-150 \mathrm{mV}$ to $-250 \mathrm{mV}$, there are a significant number of $\mathrm{Ag}$ 
NRs/NWs on the electrode surface. By controlling the deposition time and potential, the average nanowire length ranged from $900 \mathrm{~nm}$ to $3 \mu \mathrm{m}$ and the yield ranged from 5-15\%. Optimized electrochemical seed-mediated growth and the controlled formation of Ag NRs/NWs and other shaped metal nanostructures on electrode supports have potential application in chemical sensing, plasmonics, and nanoelectronics. 
CHAPTER IV

\section{RESISTIVE SWITCHING IN METAL/AG NW/POLYPHENOL/METAL MICROGAP ELECTRODES}

\subsection{Introduction}

Nonvolatile RRAM is of great interest because of their potential for nonvolatile memory and programmable switches. RRAM is a form of non-volatile memory that operates by changing the resistance of material. Resistive switching devices are two terminal electronic devices that consist of metal-insulator-metal structure, where the insulator works as a solid electrolyte. A variety of solid electrolyte have been used. Chalcogenides, binary oxides and polymeric materials are some of the examples of solid electrolytes. Different organic materials, composites, and nanomaterials have been explored which have demonstrated some remarkable improvement in memory devices. Organic materials provide many advantages, including simple device structures, low fabrication costs, printability, and flexibility. Takhee Lee and Yong Chen ${ }^{171}$ presented a review on organic resistive nonvolatile memory materials. This review mainly provides a general summary about the materials, structures, characteristics, and mechanisms of organic resistive memory devices. Present day research aims 
to achieve devices having a large on/off ratio and long retention time and thus the performance of the device could be greatly improved by incorporating an organic material over the substrate prior to the metal deposition.

Among organic materials, polymeric materials have attracted much interest. The polymers such as polyphenol and polystyrene are the insulating polymers that can be used in programmable switch. By applying a voltage bias across the electrodes of such a device, the electrical conductivity of the thin film can be changed reversibly and be retained for a sufficiently long time between a highly conductive (ON) state and a highly resistive (OFF) state. ${ }^{136}$ One mechanism involved with polymer-nanowire based device is field induced metallic filament formation. ${ }^{172,138,173}$ Winey et al ${ }^{138}$ studied resistive switching in bulk silver nanowire-polystyrene composites. The proposed mechanism to form filaments is field induced filament formation where dissociation of atoms takes place from the silver nanowire and connects with other Ag wires. This mechanism is one type of conducting filament mechanism where formation and rupture of the filament is observed on application of voltage. These wires are only a few nanometers in distance from each other. The other mechanism could be a redox reaction that induces filamentary conduction, where oxidation and reduction of metal atoms takes place and forms a conductive bridge from cathode to anode.

Our initial goal was to study electronic and sensing properties of $\mathrm{Ag}$ NWs/NRs grown by seeded growth across gaps. Since that did not work, we used different procedure developed in our group to form Ag NWs electrochemically across gaps. ${ }^{38}$ We use a simple electrochemical procedure described by our group 
previously for easy fabrication of $\mathrm{Ag} \mathrm{NW/polymer/electrode} \mathrm{junctions} \mathrm{across}$ microelectrode gap. We electropolymerized polyphenol on one side of the IDA electrode prior to electrodeposition of Ag NWs on the other side of the electrode to create an Electrode/Ag NW/polyphenol/Electrode nanojunction using the procedure previously developed by our group, resulting in a voltage dependent $\mathrm{Ag}$ NW-based switch. Later, studied the fundamentals of the Ag nanowire (NW)based resistive switching devices. The device showed unipolar switching behavior with fast speed, several cycles, low voltage, and large ON/OFF ratio, but the reproducibility of device fabrication was not optimal (about $40 \%$ success rate). During the switching cycles, the Ag NWs transform from intact NWs into chains of NPs. It is not clear if this is due to an electrochemical process, a heating process, or atomic migration in the electric field. The devices show stable switching behavior for 1000s of cycles despite the dramatic morphology change from NWs to NP chains.

\subsection{Experimental}

Electrode wiring and cleaning. Procedure is discussed in Chapter II section 2.3.3.

Electrodeposition of polyphenol and Ag deposition. Refer to Chapter II section 2.4.5 and section 2.4.6.

Characterization of the device by SEM is explained in the Chapter II section 2.3.2. 


\subsection{Results and discussion}

Scheme 4.1 shows the procedure to fabricate metal/polyphenol/Ag NW/metal devices. The first step involves electrodeposition of polyphenol by cycling 100 times on electrode $\mathrm{E} 2$ from 0 to $1.2 \mathrm{~V}$ in a solution containing $5 \mathrm{mM}$ polyphenol and $0.1 \mathrm{M} \mathrm{H}_{2} \mathrm{SO}_{4}$ on electrode E2. The device was then rinsed with nanopure $\mathrm{H}_{2} \mathrm{O}$ and dried under $\mathrm{N}_{2}$. Step 2 involves electrodeposition of $6 \times 10^{-5} \mathrm{C}$ of $\mathrm{Ag}$ on Electrode 1 using coulometry at a potential of $-0.3 \mathrm{~V}$, which took about $20 \mathrm{sec}$. As described previously, this leads to the deposition of Ag NWs that span the $5 \mu \mathrm{m}$ electrode gap and connect to the other side where polyphenol was deposited. The polyphenol at E1 electrode has a low conductivity and serves as a resistive barrier to electron transport from the Ag NW to the E2 electrode. With an increasing number of cycles of polyphenol, the polyphenol film becomes a little thicker, but mostly denser. These films typically contain defects that become filled with an increasing number of electrochemical cycles making them better barriers with increasing cycles. ${ }^{174}$ After we fabricated a device as explained in Scheme 4.1, we evaluated the device for electronic and switching.

\subsubsection{Fabrication of metal/polyphenol/Ag NW/metal device}

We obtained an I-V curve in air from $-1 \mathrm{~V}$ to $+1 \mathrm{~V}$. Figure 4.1 shows the overlay of current-potential (I-V) plots from $-1 \mathrm{~V}$ to $+1 \mathrm{~V}$ of the device before fabrication (background), after polyphenol deposition at E2, and after Ag deposition at E1. The polyphenol deposited on electrode 2 hinders the contact between the Ag wire and electrode E2, showing very low pA current, similar to the 

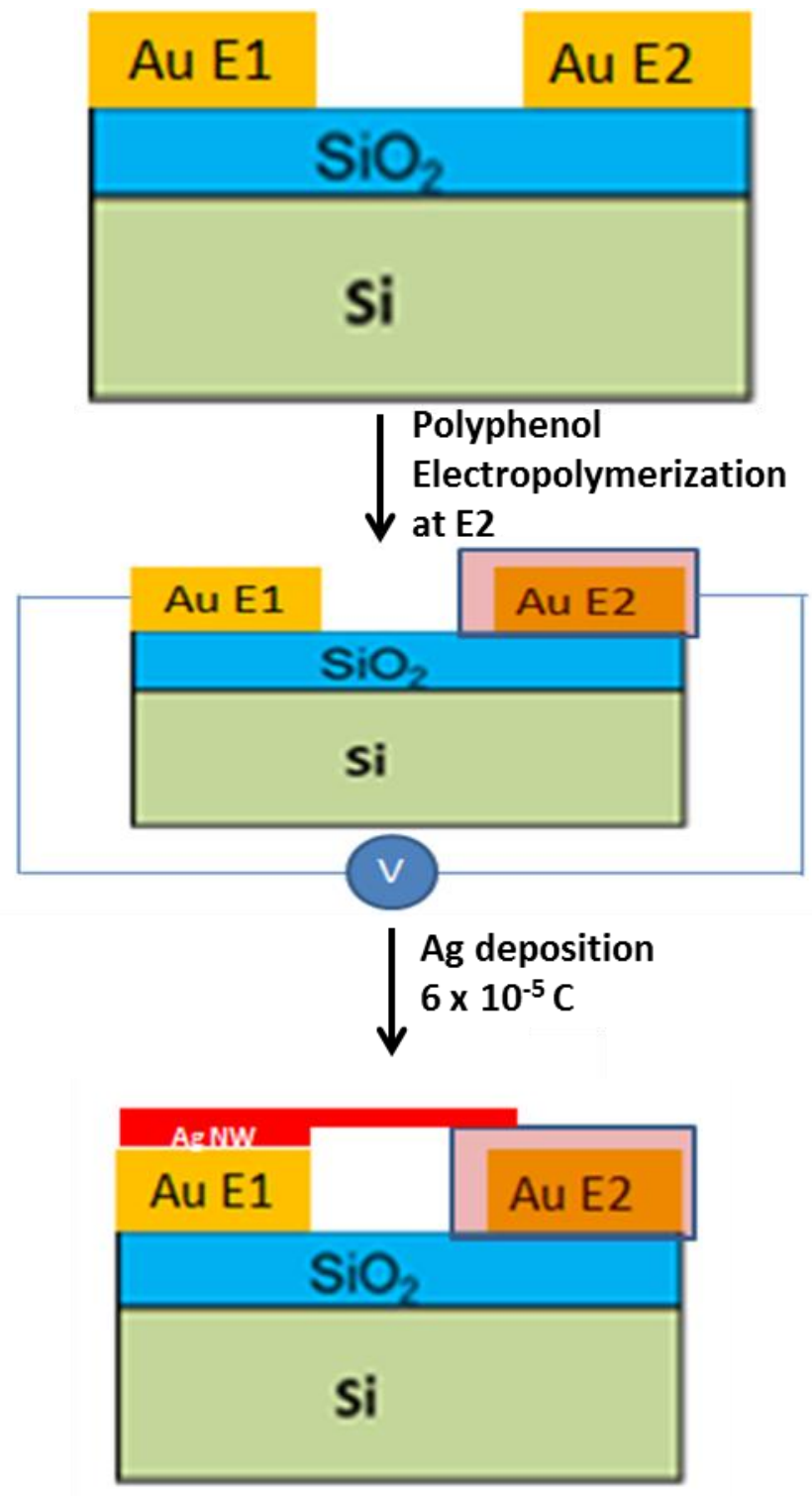

Scheme 4.1. Schematic illustrating the procedure for the fabrication of electrode(E1)/Ag NW/polyphenol/electrode (E2) devices. 


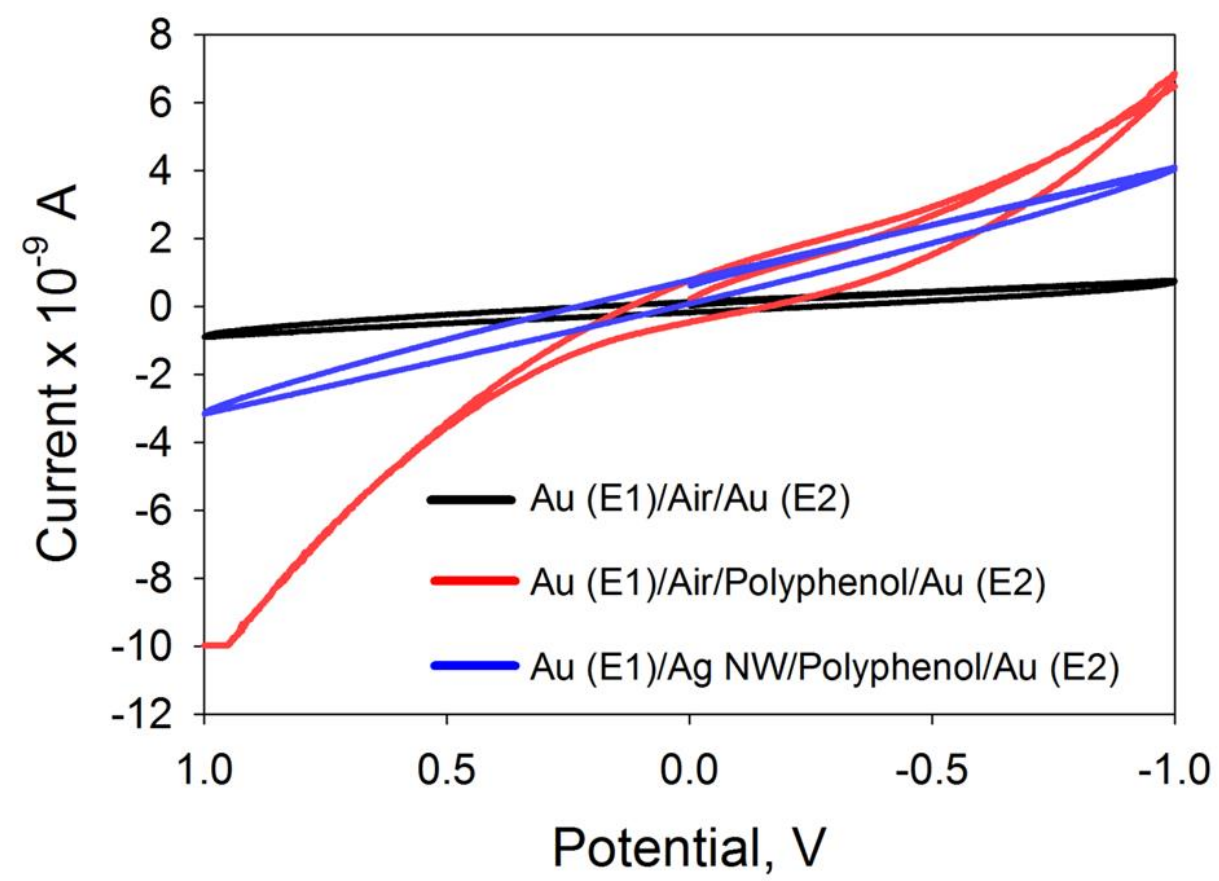

Figure 4.1. I-V plot of 1) Au E1/Air/Au E2 (Black), 2) Au E1/Air/polyphenol/Au E2 (Blue) after polyphenol deposition for 100 cycles at E2, and, 3) Au E1/AgNW/polyphenol/Au E2 (Red) after Ag deposition at E1. 
background noise level. A very increase in device current is observed after polyphenol and Ag deposition. The current is slightly above background and there is some hysteresis in the I-V curve. Figure 4.2 shows SEM images of the bare E1 Au/Air/Au E2 IDA, E1 Au/air/polyphenol/Au E2 IDA, and E1 Au/Ag NW/polyphenol/Au E2 IDA. E1/Air/E2 and E1/Air/polypheno1/E2 should have no connection but $\mathrm{E} 1 / \mathrm{Ag} / \mathrm{NW} /$ polyphenol/E2 should have a connection as shown in Figure 4.1 (red plot). The polyphenol side (E1) looks dark since it is an insulator and Ag side (E2) looks bright in the SEM images.

\subsubsection{Resistive Switching in the Presence of Humid Air}

After performing the sample preparation method shown in Scheme 4.1 and measuring the current, we carried out I-V studies at higher voltages. We scanned the device from 0 to $-5 \mathrm{~V}$ and $-5 \mathrm{~V}$ to $0 \mathrm{~V}$ as shown in Figure 4.3. The black arrows represent the forward scan and red arrows represent the reverse scan. As shown in Figure 4.3A, the device initially showed zero current from 0 to $-5 \mathrm{~V}$ during the forward scan. On the reverse scan, the device showed current spikes around $-2 \mathrm{~V}$ and a sharp current increase at $\sim-1 \mathrm{~V}$, which remained stable throughout the completion of the cycle. This rise in current shows the formation of a connection between the microelectrode gap likely between the Ag NW and E2. In Figure 4.3 B, we observed that the device was at a Low Resistance State (LRS) from 0 to $-1 \mathrm{~V}$ and switched to a High Resistance State (HRS) from $-1 \mathrm{~V}$ to $-5 \mathrm{~V}$ during the forward scan, indicated by linear I-V curve that drops dramatically in current at $\sim-1 \mathrm{~V}$. In the reverse scan, the device remained in HRS (low current) 


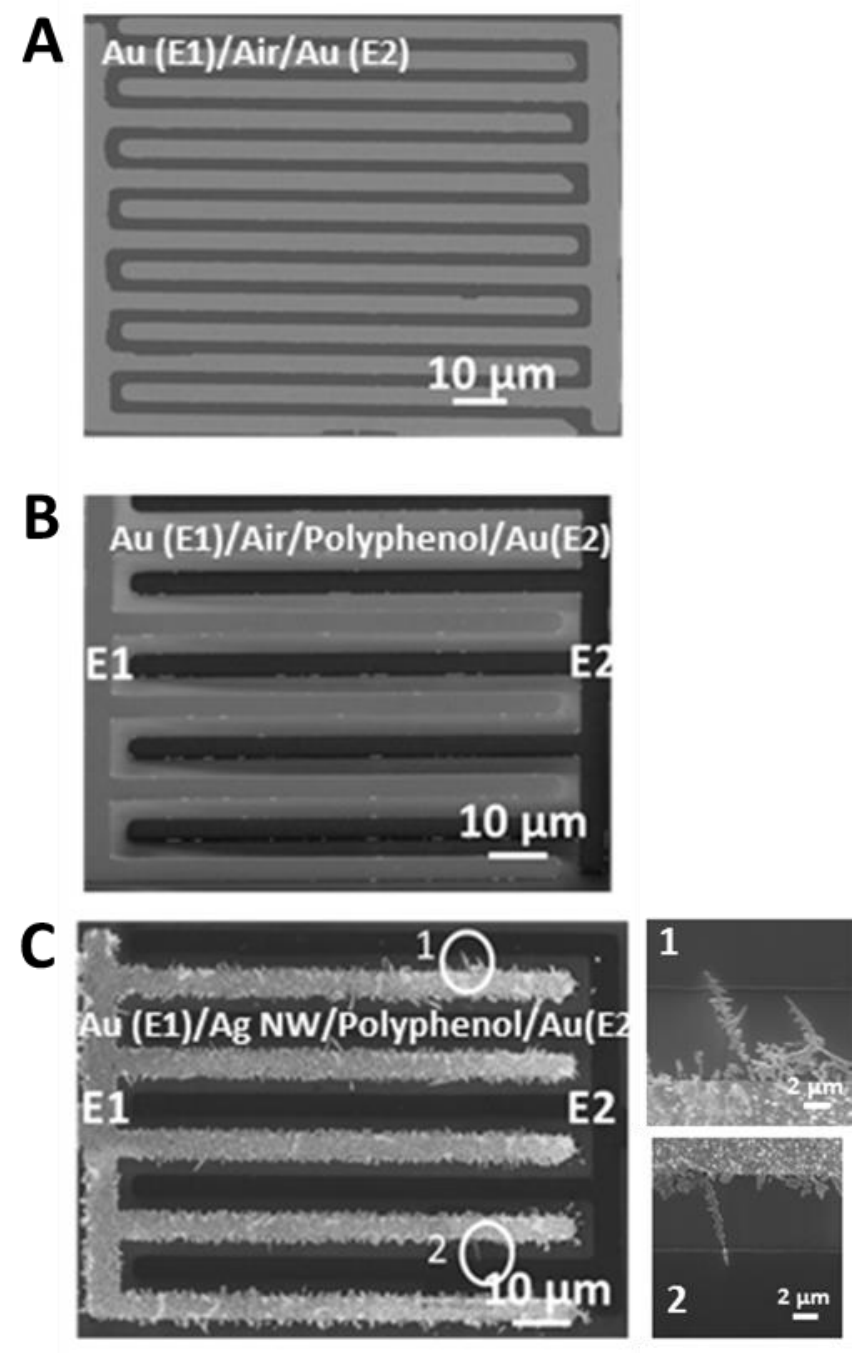

Figure 4.2. SEM images of the Au IDA device after different steps for the fabrication of electrode/polyphenol/Ag NW/electrode device. (A) E1/Air/E2 IDA. (B) E1/Air/polyphenol/E1 IDA and (C) E1/Ag NW/polyphenol/E2 IDA. 

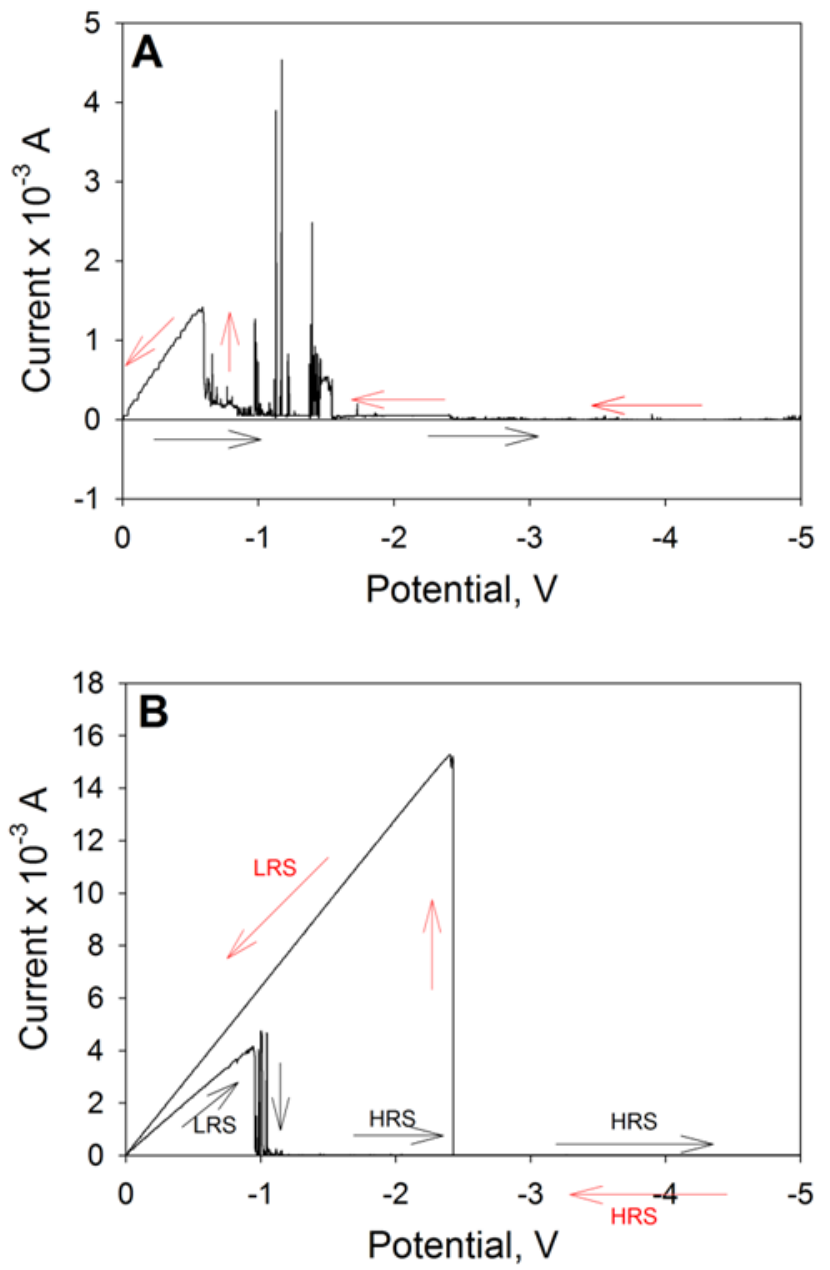

Figure 4.3. I-V plots of the electrode/Ag NW/polyphenol/electrode device scanned from $0 \mathrm{~V}$ to $-5 \mathrm{~V}$ and $-5 \mathrm{~V}$ to $0 \mathrm{~V}$. Black arrows represent forward scan and red arrows represent reverse scan. (A) Scan 1 shows a connection formed on the reverse scan. (B) Scan 2 shows good electrical behavior for resistive switching with high resistance negative of $-1.0 \mathrm{~V}$ on the forward scan and low resistance positive of $-2.5 \mathrm{~V}$ on the reverse scan. 
from $-5 \mathrm{~V}$ to $-2.5 \mathrm{~V}$ but switched to a LRS (high current) from $-2.5 \mathrm{~V}$ to $0 \mathrm{~V}$. This device shows nice unipolar resistive switching behavior that could be useful for memory applications.

After the I-V studies, we switched the device rapidly between $-0.5 \mathrm{~V}$ and -5 $\mathrm{V}$ and measured the current as a function of time at these voltages to determine if these states were stable and reversible. Ag side was kept positive and polyphenol side was kept negative when carrying out switching experiments. These voltages were chosen based on the I-V studies performed prior to the switching experiment. Figure 4.3 shows that the ON state (LRS) is at $-0.5 \mathrm{~V}$ and OFF state (HRS) is at -5 V. Figure 4.4 shows the current time plot of the device. The device shows distinct $\mathrm{ON}$ and OFF states between $-0.5 \mathrm{~V}$ and $-5.0 \mathrm{~V}$, respectively, at pulse width of 0.25 s. The current changes rapidly and is reversible for many cycles. We characterized the device by SEM following the I-V curves and rapid switching experiments as shown in Figure 4.5. Surprisingly, we no longer observe Ag NWs but instead observed group of Ag NPs forming a cone shape. Before switching we could see Ag NW as shown in Figure 4.2 C. It appears that the Ag from the Ag NWs or bulk Ag on E1 formed Ag NP arrays across the gap. Based on their appearance, this is likely due to an electrochemical process like that described in Chapter V. We performed further studies in nitrogen environment and took optical images after each step performed on the device after its fabrication to better understand what occurred. 




Figure 4.4. Current-time plot of the device after performing the I-V curves as shown in Figure 4.3. 

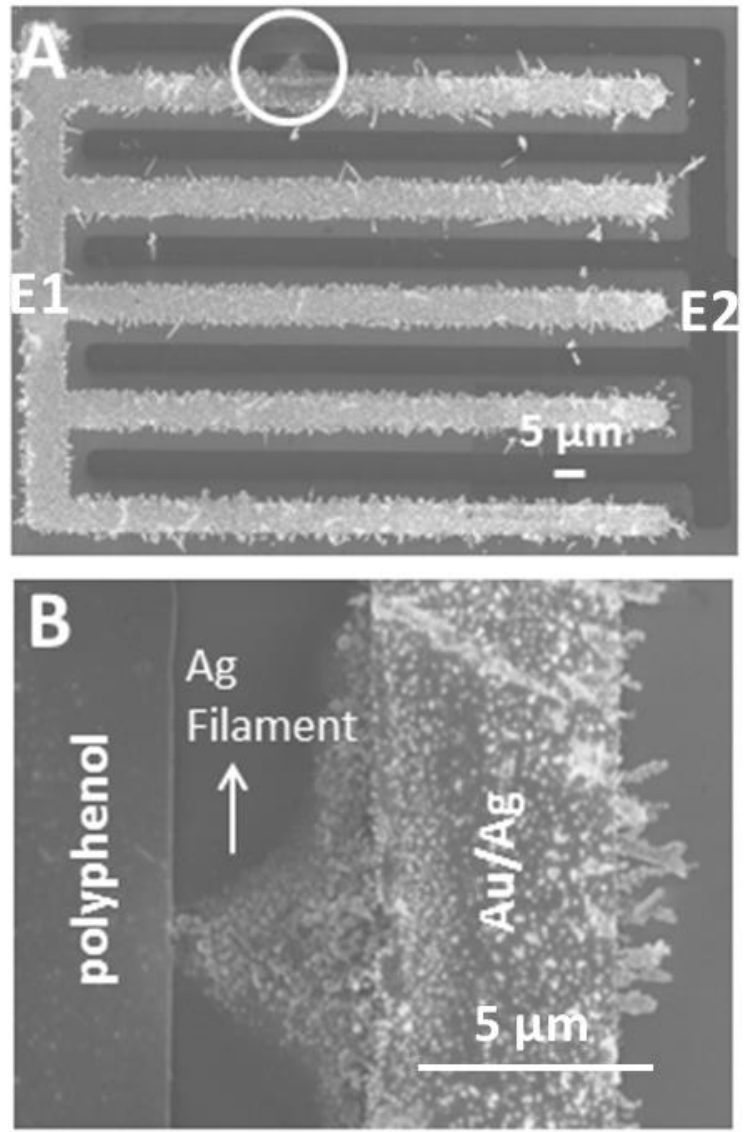

Figure 4.5. SEM images of the device after the performing I-V studies and switching cycles. 


\subsubsection{Resistive switching in the presence of nitrogen}

After the device was fabricated according to Scheme 4.1, we performed I-t studies to form the Ag NP arrays/chains. We performed current-time studies instead of I-V studies for the formation of chains in this case. We initially applied $5 \mathrm{~V}$ for $1000 \mathrm{~s}$ but did not observe any noticeable change in the current level. We applied $10 \mathrm{~V}$ thereafter for $1000 \mathrm{~s}$. We observed that the current raised up to 8 orders of magnitude around $420 \mathrm{~s}$ in air as shown in Figure 4.6.

After we observed the high current, we carried out I-V studies. Figure 4.7 shows the I-V plot from 0 to $-5 \mathrm{~V}$ and $-5 \mathrm{~V}$ to $0 \mathrm{~V}$. During the forward scan, the device shows LRS from 0 to $-2 \mathrm{~V}$ the appearance of little current spikes from $-2 \mathrm{~V}$ to $-3.5 \mathrm{~V}$, and full HRS state from $-3.5 \mathrm{~V}$ to $-5.0 \mathrm{~V}$. On the reverse scan, the device was in the HRS from $-5 \mathrm{~V}$ to $-2.2 \mathrm{~V}$ and changed to a LRS from $-2.2 \mathrm{~V}$ to $0 \mathrm{~V}$. The device showed nice unipolar resistive switching behavior similar to the first device. All of the above studies were performed in air. Figure 4.8 show I-t plot of the device with different programming speeds for 20 cycles $\mathrm{u} / \mathrm{N}_{2}$. We measured the device at $0.25 \mathrm{~s}$ and $0.025 \mathrm{~s}$. The device switched from a low current to a high current state with a potential change from $-5 \mathrm{~V}$ to $-0.5 \mathrm{~V}$, respectively, consistent with the I-V curve. The $\mathrm{ON}$ and OFF states could be defined as ' 1 ' and ' 0 ' as in digital memories, and the process to turn the device from ' 0 ' to ' 1 'or from ' 1 ' to '0'could be defined as 'write' and 'erase', respectively. We did measurements at speeds as high as $0.2 \mathrm{~ms}$. At this speed, the ON and OFF merged into one another. Our instrument is incapable of generating a pulse shorter than $0.2 \mathrm{~ms}$. 


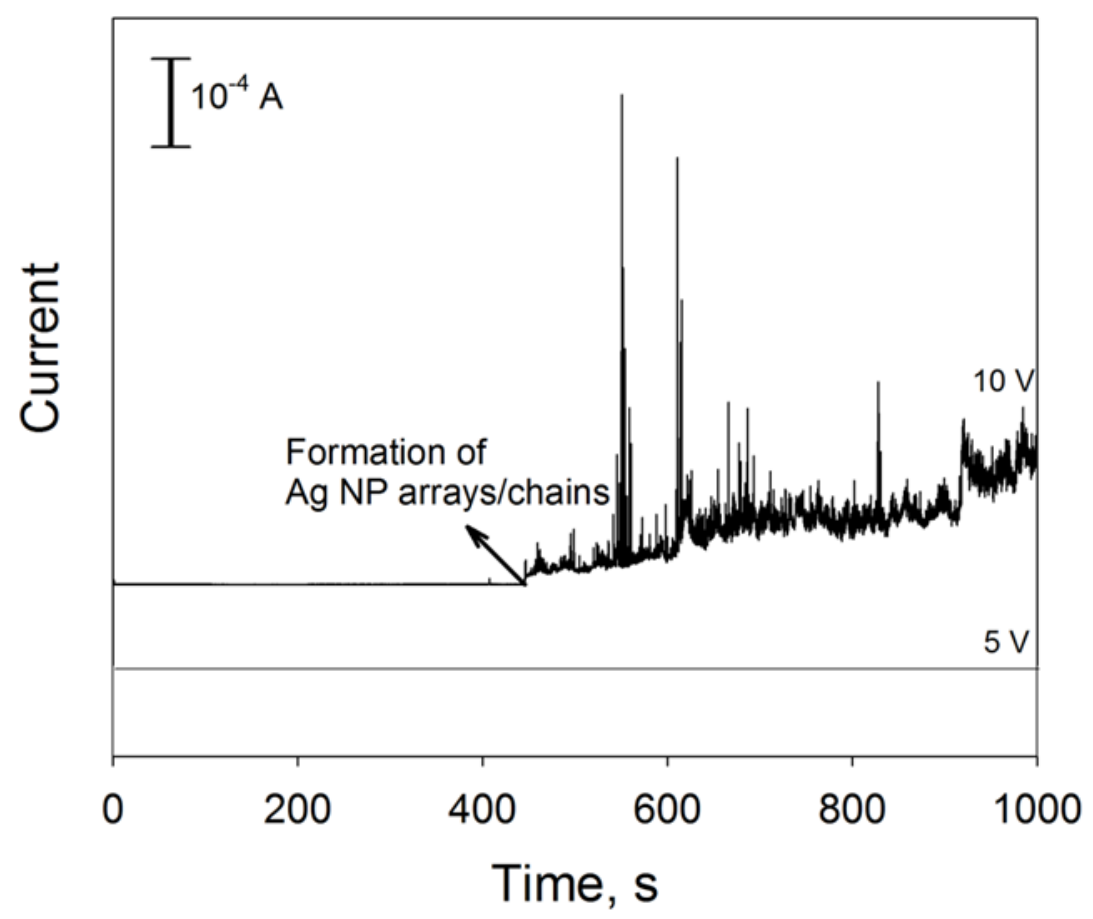

Figure 4.6. Current-time plot of an Au E1/Ag NW/Polyphenol/Au E2 device showing a current increase at the indicated voltage due to AgNP arrays spanning the electrode gap. 




Figure 4.7. I-V plot after I-t plot shown in Figure 4.6 

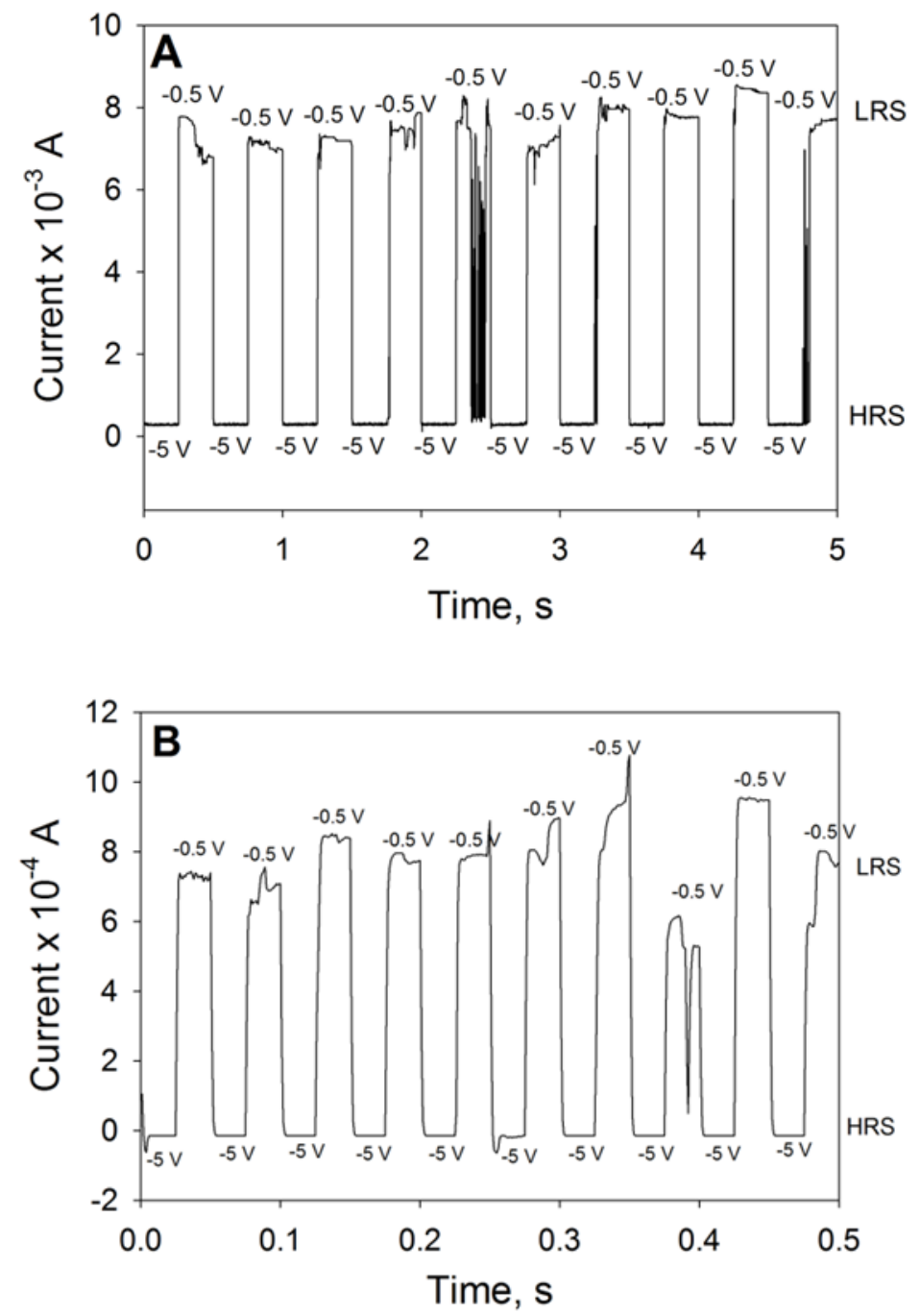

Figure 4.8. Current time plot for two different programming speeds. (A) $0.25 \mathrm{~s}$ (B) $0.025 \mathrm{~s}$. 
It is possible that this device is faster than we are able to measure. Figure 4.9 showsthe endurance behavior of the same device after performing switching experiments. It shows the first and last 20 cycles of 1000 cycles. We observed that the device showed quite stable behavior throughout the 1000 cycles. The on and off ratio was observed to be $\sim 1000$ as shown in Figure 4.10. A large ON/OFF ratio is desired as it is easier to distinguish the storage info between 1 and 0 . This electrical behavior renders these resistive switching devices an important application as NVM devices. All of these switching studies were performed under nitrogen environment. Ag filaments were formed in the presence of air. It did not form in absence of air. After characterization of the device by SEM, we observed Ag NP arrays across the $5 \mu \mathrm{m}$ electrode gap as shown in Figure 4.11. This may be resulting from oxidation or redeposition as Ag as explained in Chapter V. Figure 4.11 shows the SEM images. In Figure 4.11 D, we did not observe filaments as we observed for $4.11 \mathrm{~B}$ and $4.11 \mathrm{C}$. This NW may not be in close enough contact to E2. Alternatively, a small chain of Ag NPs could be connecting it, which could be smll enough to observe under the microscope. We believe that there could be or there could be no filament from this location. If there is any filament formed, then it would be very small to observe under microscope. 

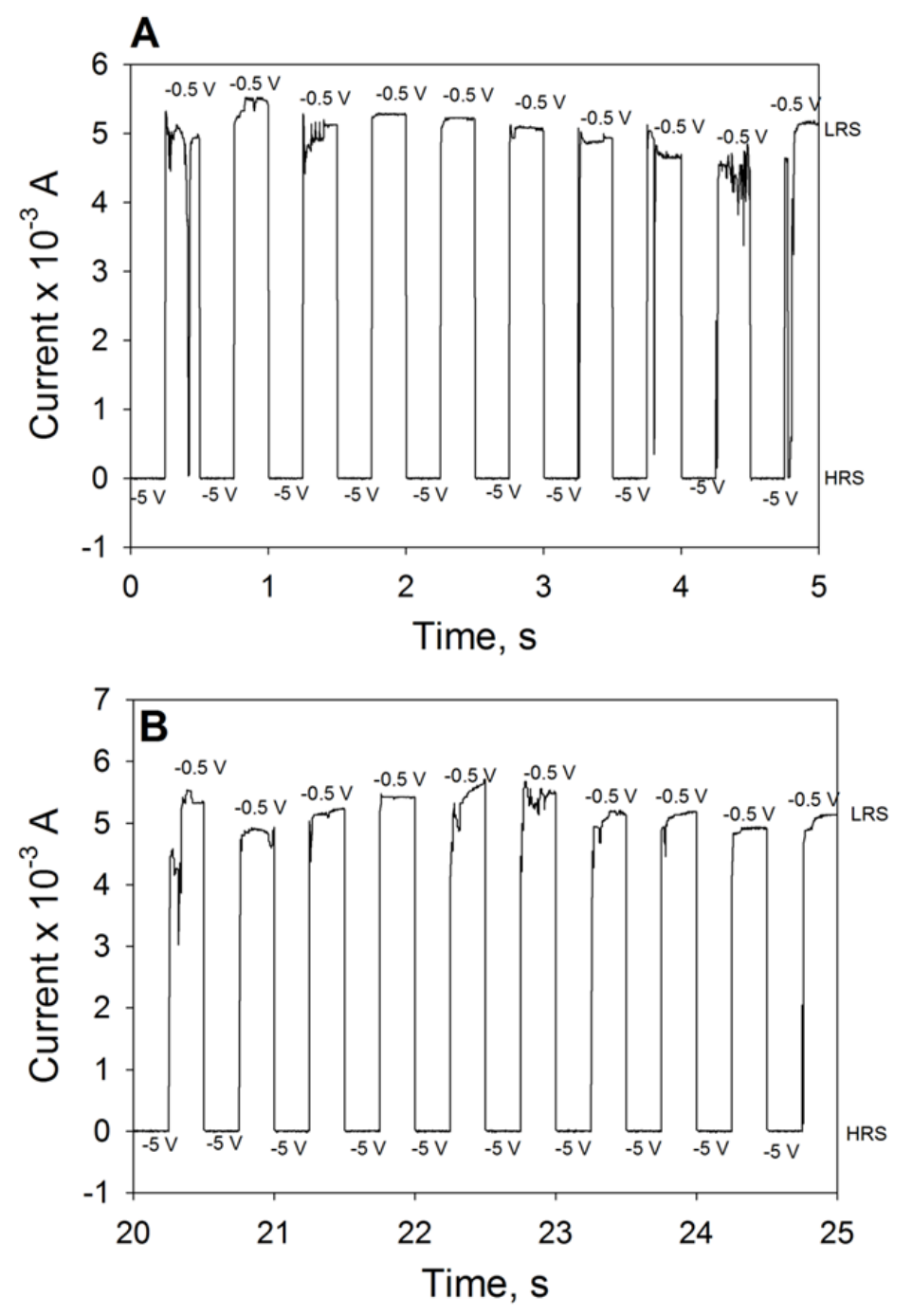

Figure 4.9. Endurance of the device after performing I-t plots shown in Figure 4.8. The device showed endurance for at least 1000 cycles. (A) first 20 cycles of 1000 cycles and (B) last 20 cycles of 1000 cycles. There is almost no change in the ON and OFF currents. 

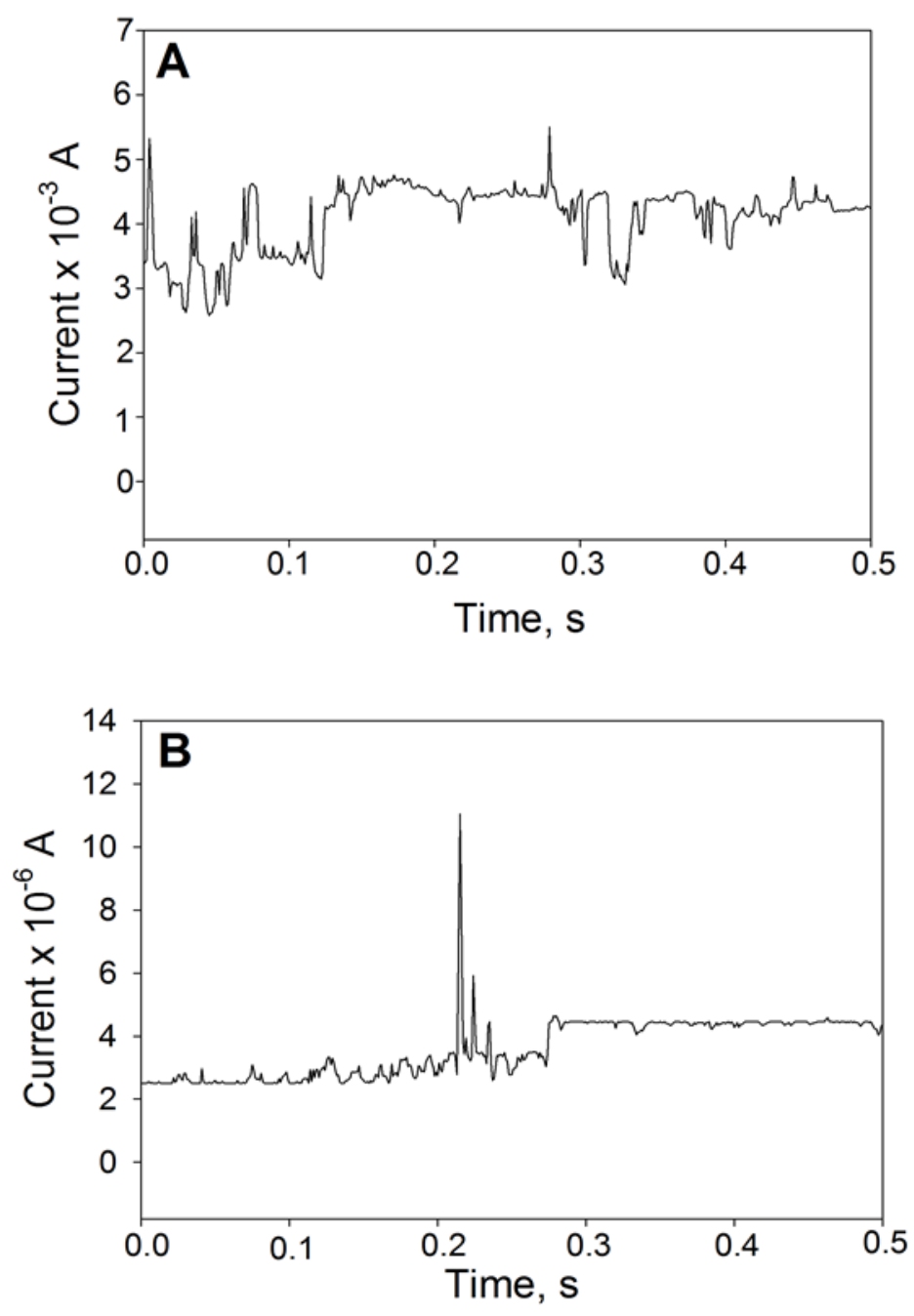

Figure 4.10. Current-time plot showing the ON (A) and OFF (B) current levels for the device. The ON/OFF current ratio is $\sim 1000$. 

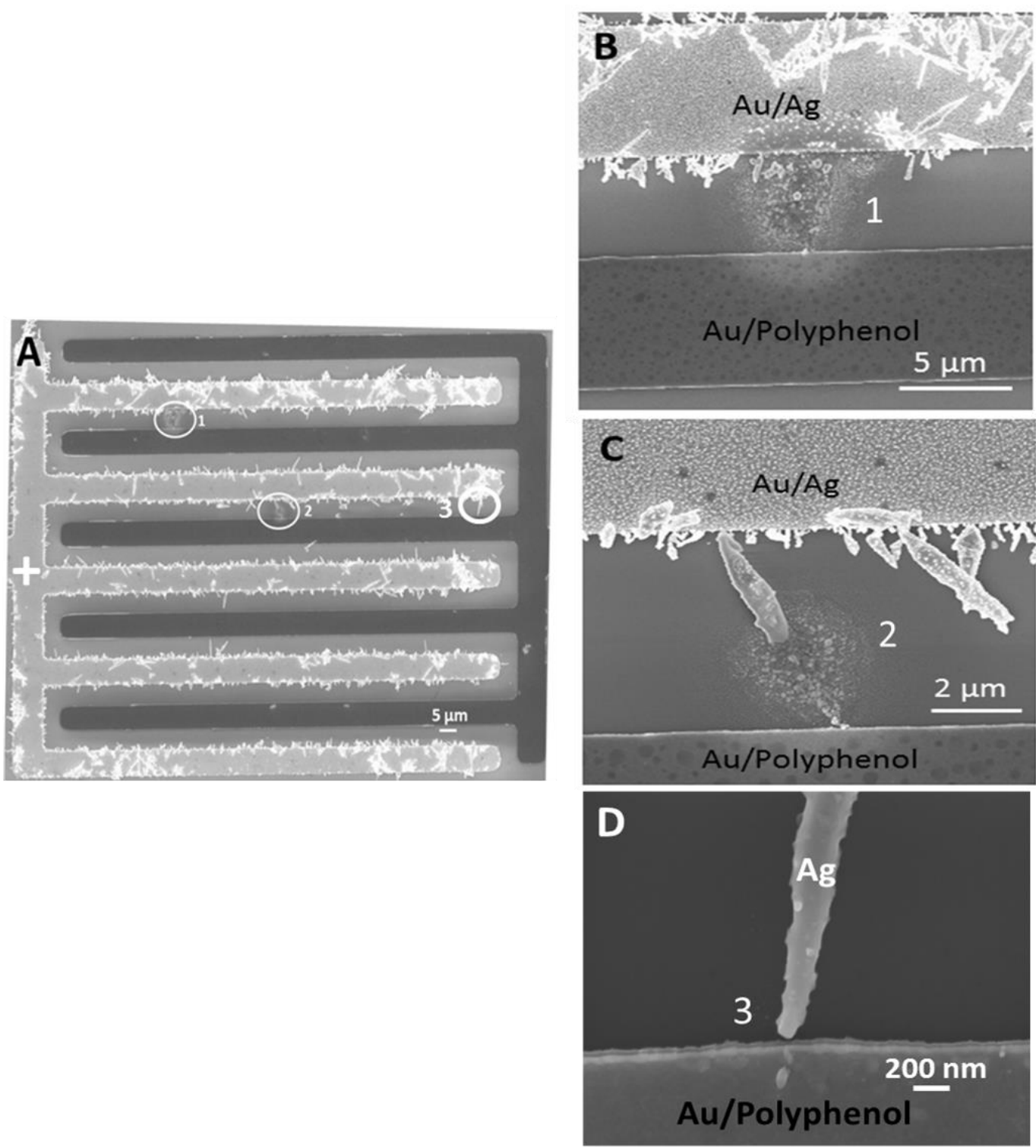

Figure 4.11. SEM images of the device after performing all switching experiments. SEM and optical images are consistent with each other, showing the Ag NP chains forming from AgNW at the locations marked by numbers 1, 2 and 3. 


\subsubsection{Possible Mechanism of AgNP Chain Formation and Resistive Switching}

It is important to understand the mechanism of chain formation and resistive switching, as it is different than our studies in Chapter V. In our study we observed chain formation in the presence humid air. The electrochemical mechanism involves oxidation of metal ions at the anode which migrate towards the cathode and becomes reduced. Reduction leads to redeposition, which eventually forms connection with the anode resulting in the formation of a cone shaped chain of Ag NPs. A field-induced filament forms due to atom dissociation from the Ag chain which forms a connection with the other electrode during switching. When the device was kept under nitrogen, the mechanism could be explained as field induced. Interestingly, these nanowires fall apart in the presence of the applied voltage. The formed Ag NP chains become the active switching materials which are very stable and reversible with the polyphenol layer.

\subsection{Conclusions}

The filament formed in the Ag NW based device possibly consists of very tiny nanospheres either coming from the Ag NWs or from Ag NWs breaking into small nanoparticle chains to form a connection. There are two potential types of mechanism acting on these devices: electrochemical and field induced filament formation. The device shows $\mathrm{ON}$ and OFF states by applying a voltage in air at room temperature, which could be further referred to as behaving like a unipolar switch where $\mathrm{ON}$ and OFF voltages occurs at same polarity. For a device to be useful in NVM applications it should have fast switching speed, high on/off ratio 
and reproducibility. Our device shows good endurance $>1000$ cycles, on/off ratio $\sim 1000$ and switch speeds of as fast as $25 \mathrm{~ms}$. In the future, we will further investigate procedures to form resistive switches from more stable Ag NWs. 


\section{CHAPTER V}

SURFACTANT-ASSISTED VOLTAGE-DRIVEN SILVER NANOPARTICLE CHAIN FORMATION ACROSS MICROELECTRODE GAPS IN AIR

\subsection{Introduction}

Over the past decade, one-dimensional (1D) metal nanoparticle (NP) assemblies have gained widespread attention due to their unique structure, interesting optical and electronic properties, and potential applications. . $^{1,42,43,175}$ These $1 \mathrm{D}$ assemblies, also known as nanochains, are of particular interest in plasmonics, ${ }^{3,4}$ nanoelectronic ${ }^{79}$ and magnetic devices. ${ }^{176}$ They have been utilized in the preparation of solar cells, ${ }^{177}$ magnetic memory devices, ${ }^{178}$ electronic switches, ${ }^{99}$ and sensors. ${ }^{44,179}$ Our group is interested in designing simple, low cost strategies to control the assembly of metallic nanostructures into $1 \mathrm{D}$ arrangements and gaining a better fundamental understanding of their electronic and optical properties in order to explore applications in nanoelectronics, sensing, and plasmonics.

There are many methods to synthesize 1D metallic NP assemblies as

described in several recent reviews. ${ }^{1,42-48}$ One of the most common methods is the use of linear templates to direct the 1D assembly. Linear template methods include hard and soft templates. Anodized aluminium oxide (AAO) membranes, polycarbonate membranes, carbon nanotubes (CNTs), amorphous carbon and 
zeolite materials are all examples of hard templates, where 1D metal nanochains are formed by filling the pores or cavities of the membrane with metal NPs or by attaching them directly to $1 \mathrm{D}$ hard materials so they can align in a $1 \mathrm{D}$ fashion. For example, Au NPs have been attached to the walls of CNTs to form 1D nanochains. ${ }^{63}$ Sometimes the hard templates are prepared by lithographic methods, such as the etched grooves on the surface of a lithographically micropatterned Si wafer. ${ }^{180}$ Soft templates mainly include biological templates and organic polyelectrolytes. DNA has been used most frequently due to its linear structure and ability to attract metal NPs electrostatically. ${ }^{59,80,181}$

Cetyltrimethylammonium bromide (CTAB)-capped Ag NPs were assembled onto a soft DNA template through electrostatic attraction, resulting in 1D Ag NP chains. ${ }^{182}$

Template free or chemical based methods involve self-assembly of NPs into 1D nanochains through a variety of inter-particle interactions. Multifunctional molecules, such as dithiols, diamines, or polymers have been used to cross-link metal NPs into 1D assembly under appropriate conditions. Also, metal NPs can assemble into 1D chain through various intermolecular forces between stabilizers attached to the NPs. Dipole-dipole interactions involving asymmetric charges on individual NPs resulted in 1D assembly following a change in the medium. Linear chains of Au NPs formed when placed in ethanol instead of water, triggered by the different polarity of the medium. ${ }^{64}$ Au NPs functionalized with an amphiphilic polymer shell formed 1D chains in solution through van Der Waals forces and 
hydrophobic interactions by adjusting the $\mathrm{DMF} / \mathrm{H}_{2} \mathrm{O}$ ratio. ${ }^{183}$ Hydrogen bonding interactions ${ }^{184}$ and electrostatic interactions have also been used to trigger the formation of Au nanochains. ${ }^{65,185}$ The 1D assemblies have been formed by adding a cross-linker, modifying the $\mathrm{pH}$, changing the solvent, or by evaporative assembly of NPs from a solution.

Other external forces can be exploited for the preparation of 1D metal NP assemblies, including electric, magnetic, or capillary forces. ${ }^{45,54}$ The dipolar attraction caused by a magnetic or electric field is one of the most common and efficient ways to form 1D nanochains. Metal NPs with magnetic properties spontaneously align into $1 \mathrm{D}$ chain structures due to the magnetic forces between the particles. ${ }^{186} 1 \mathrm{D}$ assemblies of metal NPs can be achieved by placing the NPs in an alternating current (AC) electric field through polarization of the conductive metal NPs. ${ }^{187}$ Capillary forces between NPs can result in 1D assembly during the evaporation of solvent.

The surfactant CTAB has been previously used to electrostatically assemble metal NPs into 1D array. ${ }^{41,188}$ An appropriate concentration of CTAB served as glue that linked neighboring NPs resulting in short 1D nanochains. Nogami and co-workers ${ }^{184,189}$ studied the synthesis of $\mathrm{Au}$ and $\mathrm{Ag}$ nanochains in this manner and their use in surface-enhanced Raman spectroscopy (SERS). In another study, Sau and Murphy ${ }^{66}$ explained that the CTAB molecules come close together by sharing a common layer of counterions or by interdigitation of CTAB tails from 
neighboring NPs to form 1D assemblies. The assemblies formed spontaneously from a solution of NPs.

Several groups have reported on the use of electric fields to form 1D assembly across two separated metal electrodes. Dielectrophoresis (DEP) $73-75,77,190$ involves the generation of an $\mathrm{AC}$ electric field between two electrodes in the presence of a solution of the NPs to direct the assembly. The metal NPs become polarized when placed in the electric field. The charge created on the surface of the metal NPs interacts with the non-uniform electric field and produces a dipole, where one end of the dipole experiences a stronger electric field than the other, leading to $1 \mathrm{D}$ assembly. Stellacci et al. ${ }^{75}$ reported the controlled 1D assembly of $20 \mathrm{~nm}$ Au NPs with interesting electronic properties into nano-gaps using DEP. Chains of various sized Au NPs were assembled between nanoscale and microscale electrodes using an $\mathrm{AC}$ electric field in combination with a template that the NPs could be drop-coated into. ${ }^{77}$ Metal NPs have also been assembled from a liquid suspension into micro-wire electrical connections in the form of pearl chains by DEP. ${ }^{76}$ DEP offers the opportunity to selectively place metal nanostructures inside electrically-defined gaps with good control.

Here we describe the electrodeposition of $\mathrm{Ag}$ in the presence of CTAB onto 5 micron gap Au interdigitated array (IDA) electrodes that are bare, thiolfunctionalized, or thiol-functionalized and seeded with $4 \mathrm{~nm}$ diameter Au NPs. After deposition, applying a voltage between $5 \mathrm{~V}$ and $10 \mathrm{~V}$ in air for 0 to $1000 \mathrm{~s}$ resulted in one-dimensional (1D) Ag NP chains spanning across the IDA gap. The 
Ag NP chains form on IDAs functionalized with thiols and Au NP-seeded at about $5 \mathrm{~V}$ and at $10 \mathrm{~V}$ for the other non-seeded surfaces. Ag NP chains do not form at all up to $10 \mathrm{~V}$ when IDAs are treated with ozone or water soaking to remove possible $\mathrm{CTA}^{+}$ions from the surface, when $\mathrm{Ag}$ deposition takes place in the absence of CTAB, or when the voltage is applied under dry $\mathrm{N}_{2}$ (low humidity). Chain formation occurs by Ag moving from the positive to negative electrode. Coating the devices with a negatively-charged surfactant, sodium dodecyl sulfate, also results in Ag NP chains by Ag moving from the positive to the negative electrodes, which confirms that the chains form by electrochemical oxidation at the positive electrode and deposition at the negative electrode. The surfactant ions and the thin layer of water present in the humid environment facilitate this electrochemical process. (Results have been published. Shah et al. ACS Nano, 2015, 9, 10278-10286)

Our group previously used an electrochemical approach to grow 1D Ag NWs across electrode gaps to form electrode/Ag nanowire/molecule/electrode junctions ${ }^{38}$ for potential use in electronic-based sensing, molecular electronics, or combined electronic and surface-enhanced Raman spectroscopy measurements. ${ }^{191,192}$ In this work we electrochemically deposited Ag onto $5 \mu \mathrm{m}$ gap Au interdigitated array (IDA) electrodes or a $5 \mu \mathrm{m}$ gap Au two electrode device (TED) as in our previous work, but in the presence of CTAB, which led to Ag NPs on the IDA electrodes instead of NWs. Subsequent application of 5-10 V to the IDA in air led to the formation of $1 \mathrm{D}$ Ag NP chains connecting the electrode 
gaps. The method is unique in that it occurs in humid air instead of solution and is more similar to electrochemically-driven Ag filament-based resistive switches, ${ }^{103,110,117,193,194}$ with the surfactant acting as electrolyte. The voltage required for Ag nanochain formation is relatively low and depends on the treatment of the electrodes, the electrode gap, and amount of CTAB present during Ag electrodeposition.

\subsection{Experimental}

Electrode wiring and cleaning procedure. Refer to Chapter II section 2.2.3.

Au IDA/TED electrode modification. Refer to Chapter II section 2.2.4

Electrodeposition of Ag on 5 micron gap electrodes using cyclic

voltammetry. Refer to Chapter II. section 2.4.1.

Electrodeposition of Ag on 5 micron gap electrodes using

chronocoulometry. Refer to Chapter II section 2.4.2.

Ag nanoparticle chain formation. Refer to Chapter II. section 2.4.3.

Humidity control. Refer to Chapter II section 2.4.9.

Characterization was performed by SEM and dark field spectroscopy as described in Chapter II section 2.3.2 and section 2.3.5.

\subsection{Results and Discussion}

\subsubsection{Electrodeposition of Ag on Au IDA devices}


As described in the Experimental Section (Chapter II), Ag was electrodeposited onto bare $\mathrm{Au}, \mathrm{Au} / \mathrm{dithiol}$, or $\mathrm{Au} / \mathrm{dithiol} / \mathrm{Au}$ NPs in the presence of cetyltrimethylammonium bromide $(\mathrm{CTAB})$ at a potential of $-0.2 \mathrm{~V}$ for $1-2 \mathrm{~h}$ as needed on one or both leads of the Au IDA or TED to deposit about $3.0 \times 10^{-5}$ Coulombs of Ag, based on stripping voltammetry. After electrodeposition, we always measured the current between the two terminals of the Au IDA in room air as a function of voltage from $+1 \mathrm{~V}$ to $-1 \mathrm{~V}$ as shown in Figure 5.1. The current of the $\mathrm{Au} /$ dithiol/Au NPs device after Ag electrodeposition is at the 20-30 pA level and almost identical to an Au IDA device without any treatment, revealing that there was no connection made across the gap of the device during Ag deposition. The bare $\mathrm{Au}$ and $\mathrm{Au} /$ dithiol devices all showed the same behavior.

Figure 5.2 shows SEM images of a bare Au IDA device before (Frame A and C) and an Au/dithiol/Au NPs device after Ag deposition (Frame B and D). The SEM images confirm that Ag deposited onto the IDA device and that there was no Ag directly connected across the microgaps, explaining the pA level current in the I-V curve in Figure 5.1.

\subsubsection{Formation of one-dimensional Ag Nanoparticle chain formation across microelectrode gaps in air}

Figure 5.3 shows two-electrode current-time plots at different voltages across the IDA for bare $\mathrm{Au}$ (Figure 5.3A), Au/dithiol (Figure 5.3B), and Au/dithiol/Au NPs

(Figure 5.3C) IDA devices that had Ag electrodeposited on them in the presence of 


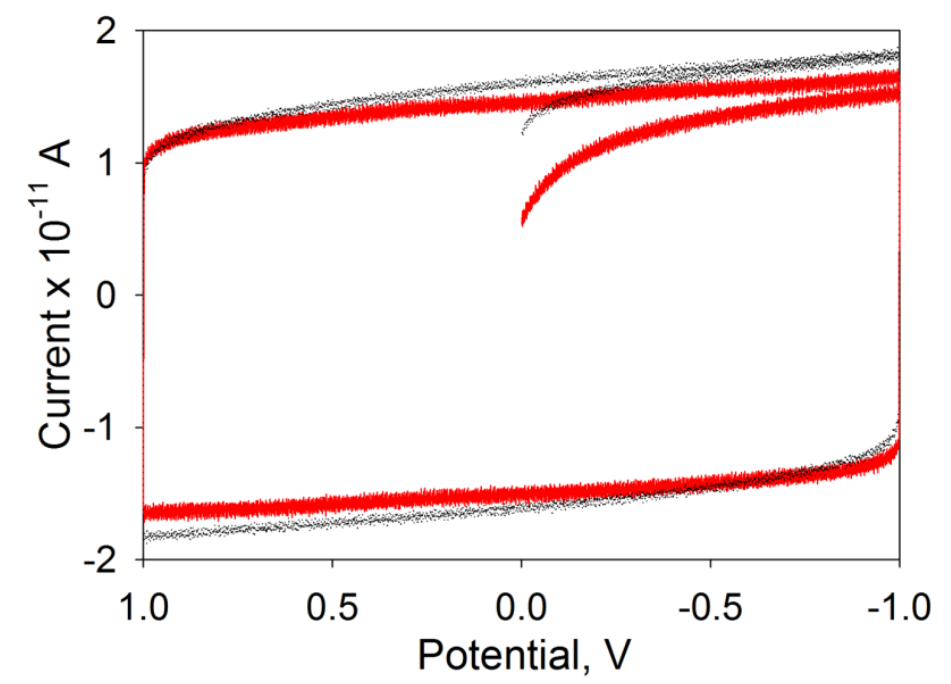

Figure 5.1. I-V curve of an untreated bare Au IDA device (grey) and $\mathrm{Au} /$ dithiol/Au NPs IDA device after Ag electrodeposition (red). The fact that the current is the same as the bare Au IDA device after Ag electrodeposition shows that the Ag did not make a connection across the 5 micron IDA gap. 

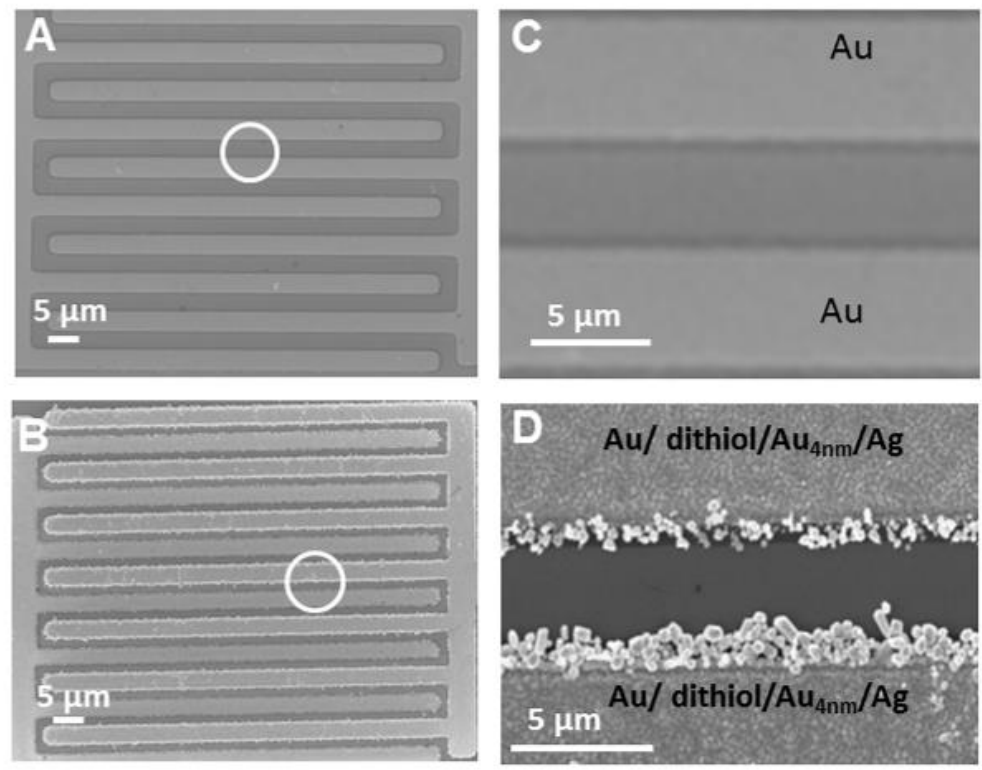

Figure 5.2. SEM images of (A,C) Bare Au IDA device and (B,D) Au/dithiol/Au NPs IDA device after Ag electrodeposition on each side. There is no obvious connection between the two electrodes of the IDA after Ag deposition, which explains the pA level current in the I-V curve of Figure 5.1. The circled regions in $\mathrm{A}$ and $\mathrm{B}$ are the regions shown in the magnified images of $\mathrm{C}$ and $\mathrm{D}$. 


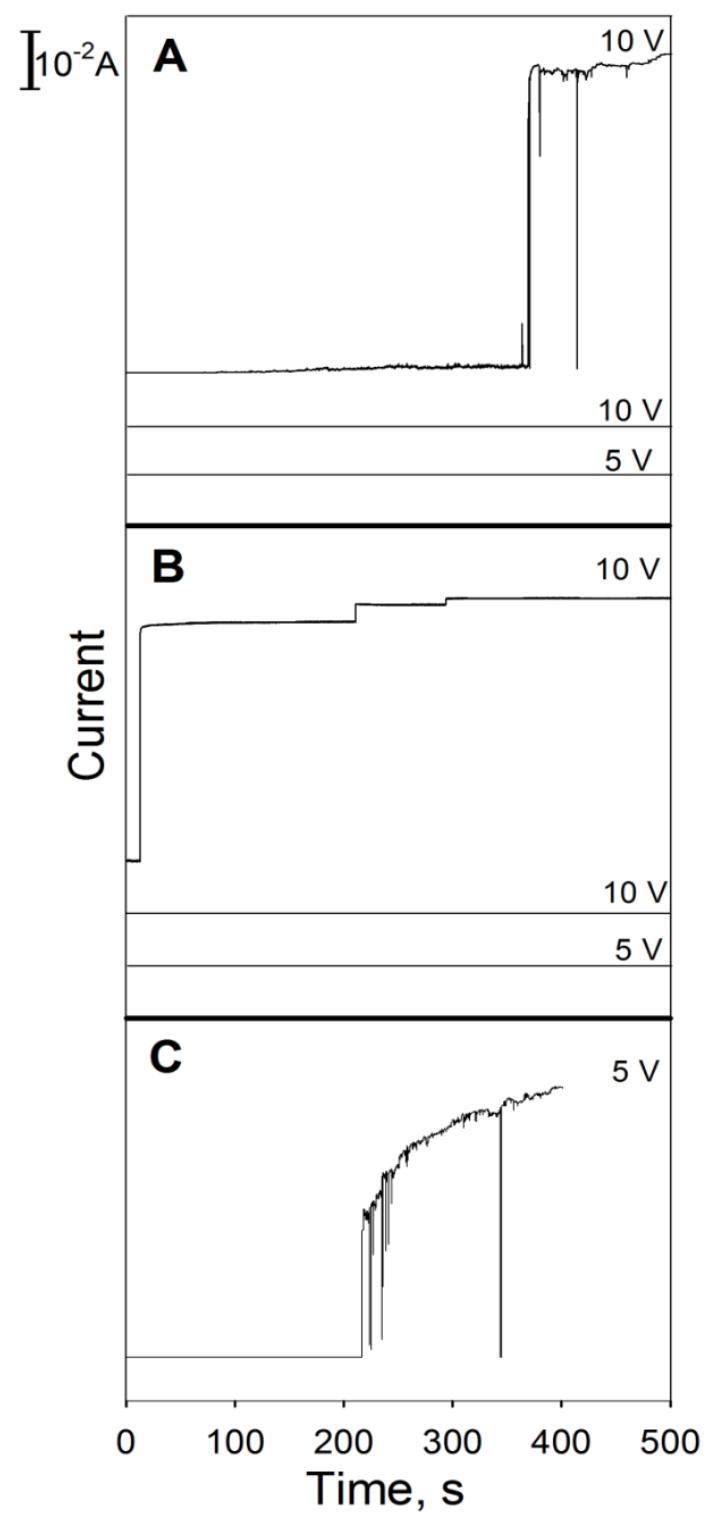

Figure 5.3. Current-time plots obtained at the indicated voltages on IDA devices with $\mathrm{Ag}$ deposited in the presence of $\mathrm{CTAB}$ on both sides of (A) bare $\mathrm{Au},(\mathrm{B})$ $\mathrm{Au} / \mathrm{dithiol}$, and (C) Au/dithiol/Au NPs devices. 
$\mathrm{CTAB}$. The bare $\mathrm{Au}$ and $\mathrm{Au} /$ dithiol samples showed a flat 0 current for the first run of $500 \mathrm{~s}$ at $5 \mathrm{~V}$. At $10 \mathrm{~V}$, the current increased to the $10^{-3} \mathrm{~A}$ level (from $\mathrm{pA}$ initially) after $900 \mathrm{~s}\left(500 \mathrm{~s} 1^{\text {st }}\right.$ run and $400 \mathrm{~s} 2^{\text {nd }}$ run $)$ and $500-550 \mathrm{~s}\left(500 \mathrm{~s} 1^{\text {st }}\right.$ run and $50 \mathrm{~s} 2^{\text {nd }}$ run) for the bare $\mathrm{Au}$ and $\mathrm{Au} /$ dithiol IDAs, respectively. In contrast, the $\mathrm{Au} / \mathrm{dithiol} / \mathrm{Au}$ NPs IDA sample increased in current to the $10^{-3} \mathrm{~A}$ level after about $200 \mathrm{~s}$ at $5 \mathrm{~V}$ on the first run. The current was spiky initially with drifting in some cases, but eventually stabilized and increased by $\sim 8$ orders of magnitude for all samples, but at different voltages and time, depending on the sample treatment. Figure 5.4 shows SEM images of the different IDA devices after Ag deposition in the presence of $\mathrm{CTAB}$ and application of $5 \mathrm{~V}$ or $10 \mathrm{~V}$ as shown in Figure 5.3. The images also show which electrodes were positive $(+)$ and negative $(-)$ during the applied voltages. From the images, it is clear that the current increased in the Figure 5.3 I-t plots due to the formation of Ag NP 1D chains across the $5 \mu \mathrm{m}$ gap, leading to a conductive pathway for electrons. The chains are wider and some Ag NP material is missing on the $(+)$ electrode, revealing that the formation of the chains occurred by movement of Ag from the $(+)$ electrode towards the (-) electrode. The chains look qualitatively similar other than there is a wider base for the electrodes without the Au NP seeds, although that may not necessarily be reproducible. We also carried out experiments to deposit Ag on one side of the device to determine if we would still observe Ag NP chain formation. Figure 5.5 shows current-time plots of devices at $5 \mathrm{~V}$ and $10 \mathrm{~V}$ with $\mathrm{Ag}$ deposited in the presence of CTAB on only one side of the Au IDA for bare Au (A), Au/dithiol (B), 

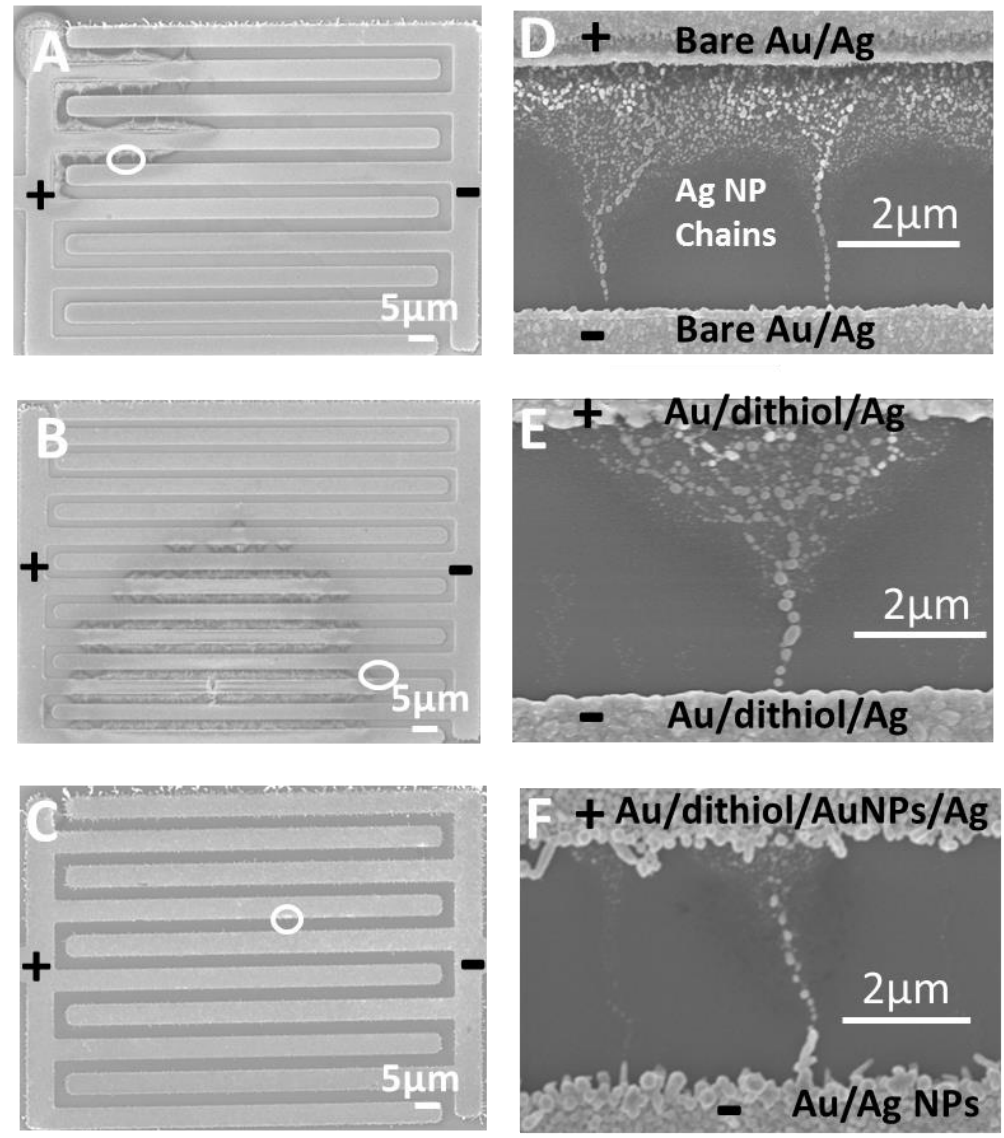

Figure 5.4. SEM images of various treated IDAs after Ag deposition in the presence of CTAB and application of an applied voltage for various times. (A,D) bare $\mathrm{Au} / \mathrm{Ag}$ IDA after applying $5 \mathrm{~V}$ and $10 \mathrm{~V}$ as shown in Figure 5.3A. (B,E) $\mathrm{Au} /$ dithiol/Ag IDA after applying $5 \mathrm{~V}$ and $10 \mathrm{~V}$ as shown in Figure 5.3B. (C,F) $\mathrm{Au} /$ dithiol/Au NPs/Ag IDA after applying $5 \mathrm{~V}$ as shown in Figure 5.3C. Chains of Ag NPs formed across the electrode gap, explaining the large 8-9 order of magnitude increase in current in the I-t plots in Figure 5.3. The circled areas are the regions that are expanded in the adjacent SEM images to the right. 




Figure 5.5. $\mathrm{Ag}$ deposition with $\mathrm{CTAB}$ on one side of the device. (A) Bare $\mathrm{Au} / \mathrm{Ag}$, (B) Au/dithiol/Ag, and (C) Au/dithiol/Au NPs/Ag. The electrode with dithiol, Au seeds, and $\mathrm{Ag}$ showed spikes corresponding to $\mathrm{Ag}$ NP chain formation after applying $10 \mathrm{~V}$ with the Ag side positive. 
and $\mathrm{Au}$ /dithiol/Au NPs (C) devices, where the side with Ag was the + terminal. There was no current for up to $1000 \mathrm{~s}$ at $5 \mathrm{~V}$ or $10 \mathrm{~V}$ for bare $\mathrm{Au}$ and Au/dithiol electrodes, while the $\mathrm{Au} /$ dithiol/Au NPs device showed several current spikes at 10 V during the $1000 \mathrm{~s}$.

The SEM images in Figure 5.6A and 5.6B correspond to the device with the current spikes in Figure 5.5C. These spikes led to the formation of Ag NP chains across the gap similar to with Ag deposited on both sides. Interestingly, this result shows that when Ag deposition is only on one side of the device, Ag NP chains form at higher voltages compared to with Ag deposited on both sides. The higher voltage requirement led to $\mathrm{Ag} \mathrm{NP}$ chain formation only on the $\mathrm{Au} / \mathrm{dithiol} / \mathrm{Au}$ NPs/Ag (1 side) IDA devices. There was no chain formation within $10 \mathrm{~V}$ on the bare $\mathrm{Au} / \mathrm{Ag}$ or $\mathrm{Au} /$ dithiol/Ag samples (Ag on 1 side). Also, a positive voltage had to be applied to the side with Ag to produce chains.

\subsubsection{Real-time dark field microscopy snap shots of Au two electrode device}

We obtained dark-field microscopy images of Ag NP chain formation across the gap of a two electrode device (TED) on glass with Ag deposited onto $\mathrm{Au} /$ dithiol/Au NPs on just one side. The top of Figure 5.7 shows the current-time plot of the device at $10 \mathrm{~V}$ (Ag side positive) and the bottom part shows real-time dark-field microscopy images of the device that correspond to the current-time plot at different times. This plot showed a very small increase in current around $60 \mathrm{~s}$ 

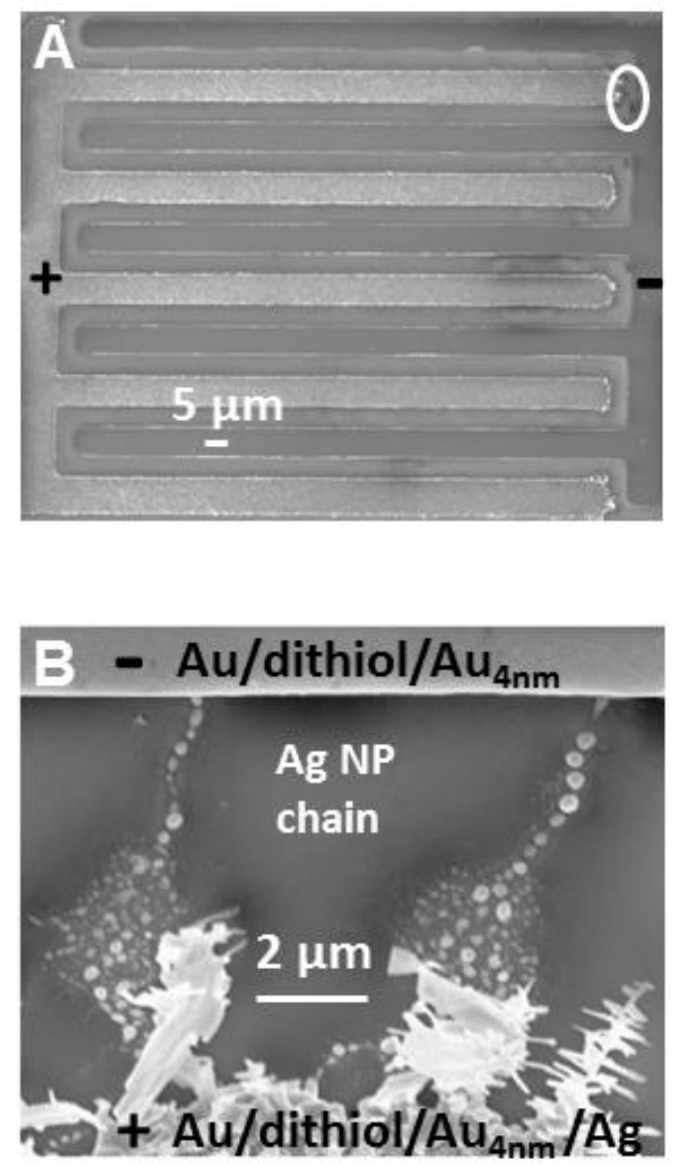

Figure 5.6. (A) SEM image of an IDA device with Au/dithiol/Au NPs/Ag on one side after application of $10 \mathrm{~V}$ (Ag side positive) as shown in Figure 5.5C. (B) Zoomed-in image of circled part in (A). 

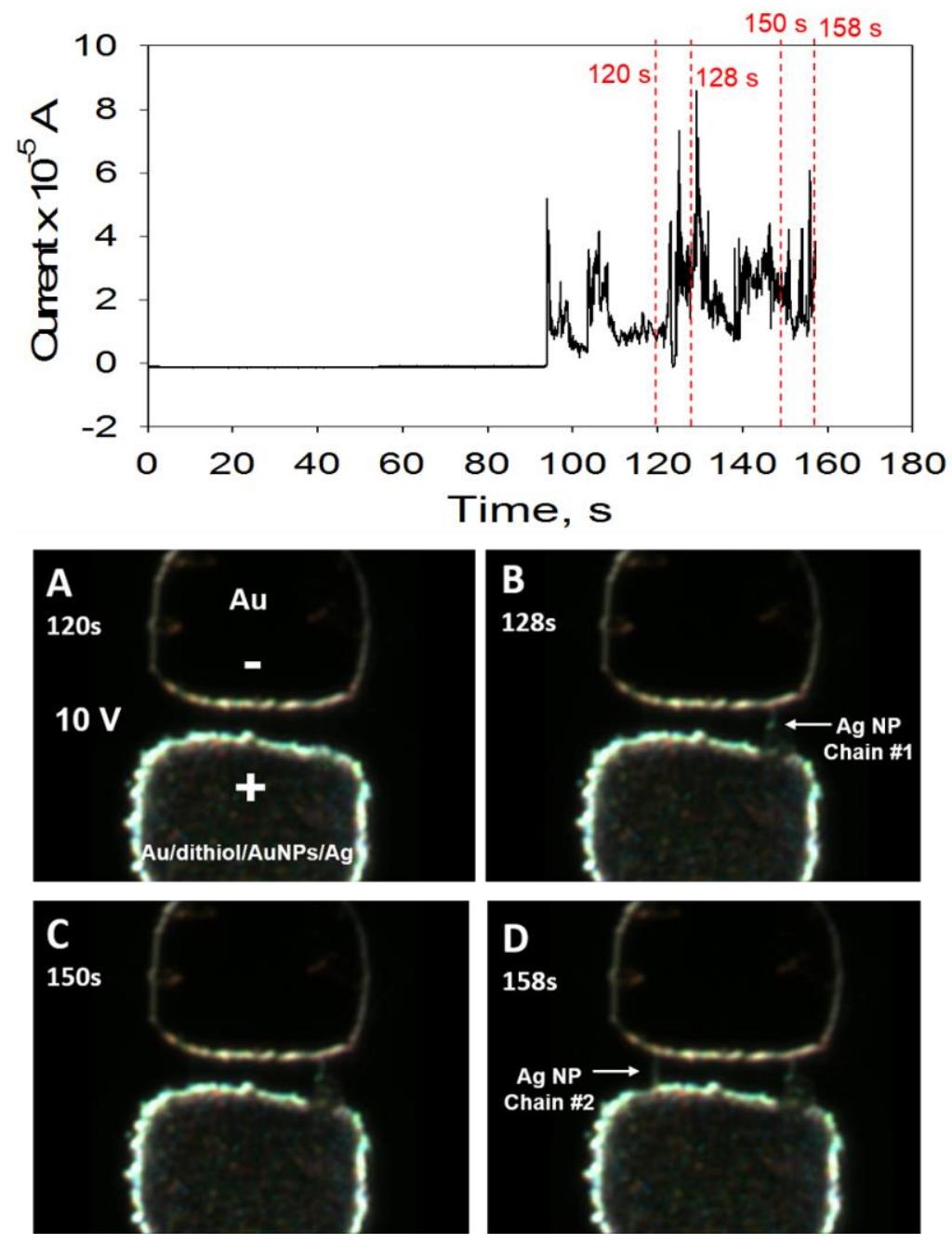

Figure 5.7. Current-time plot at $10 \mathrm{~V}$ (top) and corresponding dark-field microscopy images at (A) $120 \mathrm{~s}$, (B) $128 \mathrm{~s}$, (C) $150 \mathrm{~s}$, and (D) $158 \mathrm{~s}$ of a two electrode device on glass with bare $\mathrm{Au}$ on the negative electrode and $\mathrm{Au} / \mathrm{dithiol} / \mathrm{Au}$ NPs/Ag on the positive electrode as indicated in (A). The current increases well before the Ag NP chain can be visualized by dark-field microscopy due to the limited optical resolution. The Ag NP chains start off small and grow with time for 25-30 s until they are visible by dark-field microscopy. 
with no change in the image. A bigger increase in current to about $0.5 \times 10^{-5}$ to 1.0 $\mathrm{x} 10^{-5} \mathrm{~A}$ occurred after $95 \mathrm{~s}$ that was spiky and a bit unstable. This likely corresponds to the beginning formation of an Ag NP chain across the gap, but the size was too small or not developed enough to observe by optical dark-field microscopy. The Ag NP chain was not observed until about $26 \mathrm{~s}$ later at $121 \mathrm{~s}$ and was much clearer at $128 \mathrm{~s}$ as shown in Figure 5.7B. Figure 5.7A shows the device at $120 \mathrm{~s}$ right before the chain became visible. At $128 \mathrm{~s}$ the current was still unstable but hovering closer to $2.0 \times 10^{-5} \mathrm{~A}$. About 23 seconds later a second $\mathrm{Ag}$ NP chain was observed faintly but more clearly at $158 \mathrm{~s}$ as shown in Figure 5.7D (Figure 5.7C shows the image at $150 \mathrm{~s}$ right before the second chain was observed). Overall, this data shows that the first chain started forming at $95 \mathrm{~s}$, corresponding to the first step in the current-time plot, but was not visible until 121 s. A current jump occurred as the first Ag NP chain became visible. The current was then spiky and unstable and actually decreased a bit. Another small local current jump occurred as the second chain became visible. The current seemed to increase in interesting step-like jumps that may correspond to the number of chains, but there were also several spikes and unstable current throughout, indicating that the chain formation is a dynamic process. Figure 5.8 shows more snap shots at times closer to the observation of the two chains and Figure 5.9 shows the current-time plot with all of the times marked that correspond to the snap shots. Visualization of each chain occurs over a short time $\operatorname{span}(\sim 1 \mathrm{~s})$ and clearly occurs by movement of $\mathrm{Ag}$ from the positive to the negative electrode 

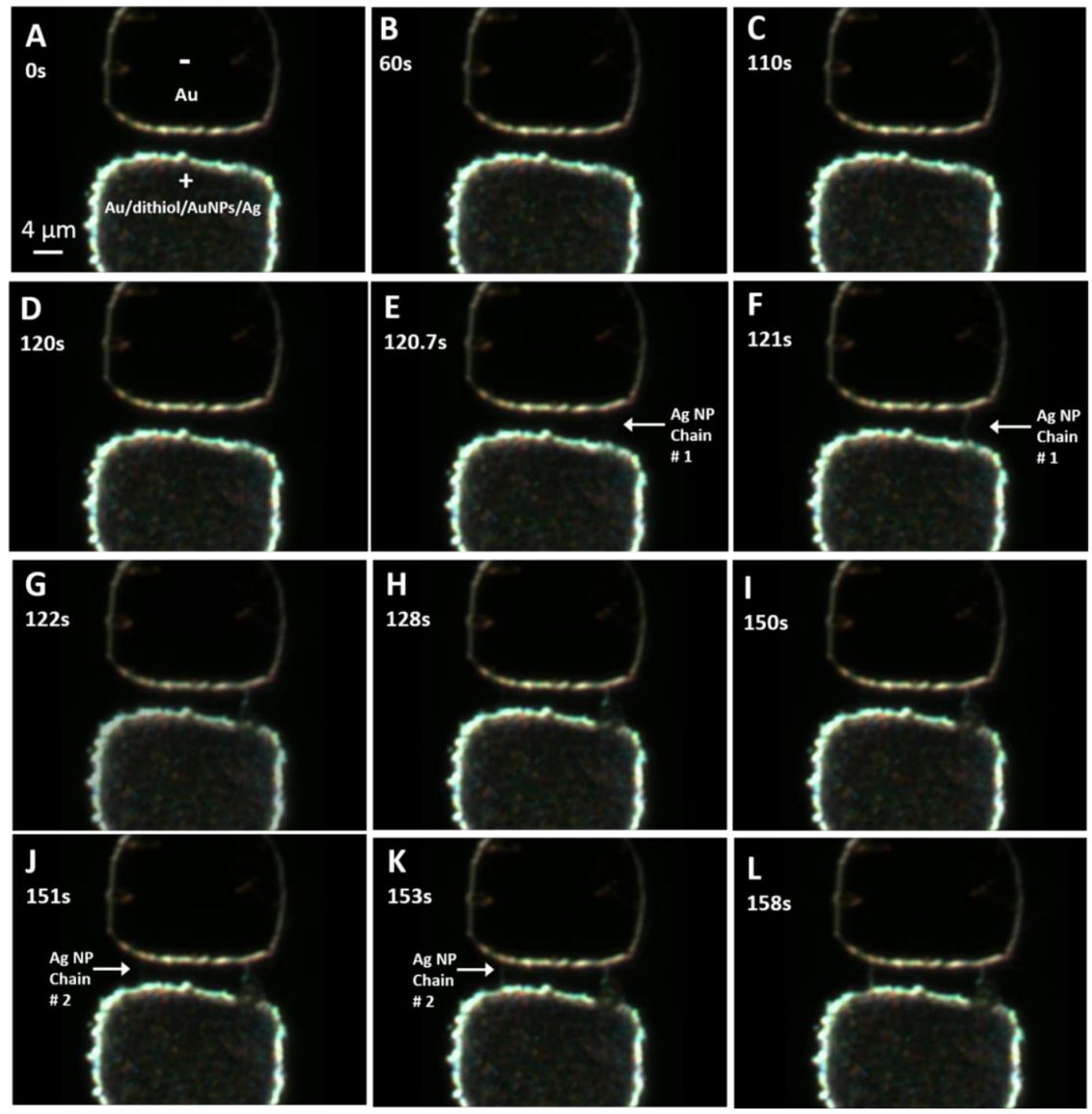

Figure 5.8. Real-time dark-field microscopy snap shots of an Au two electrode device with $\mathrm{Au} / \mathrm{dithiol} / \mathrm{Au} \mathrm{NPs} / \mathrm{Ag}$ on one side held at $10 \mathrm{~V}$ for various amounts of time. The times indicated correspond to the current-time plot shown in Figure 5.9. An initial jump in current occurred about $25 \mathrm{~s}$ before the appearance of Ag NP chain 1. A jump in current appeared during the appearance of chain 1 and chain 2 . Chain formation occurs by movement of Ag from the positive electrode to the negative electrode as indicated by the loss of Ag on the positive side. 


\section{Chain 1 Chain 2}

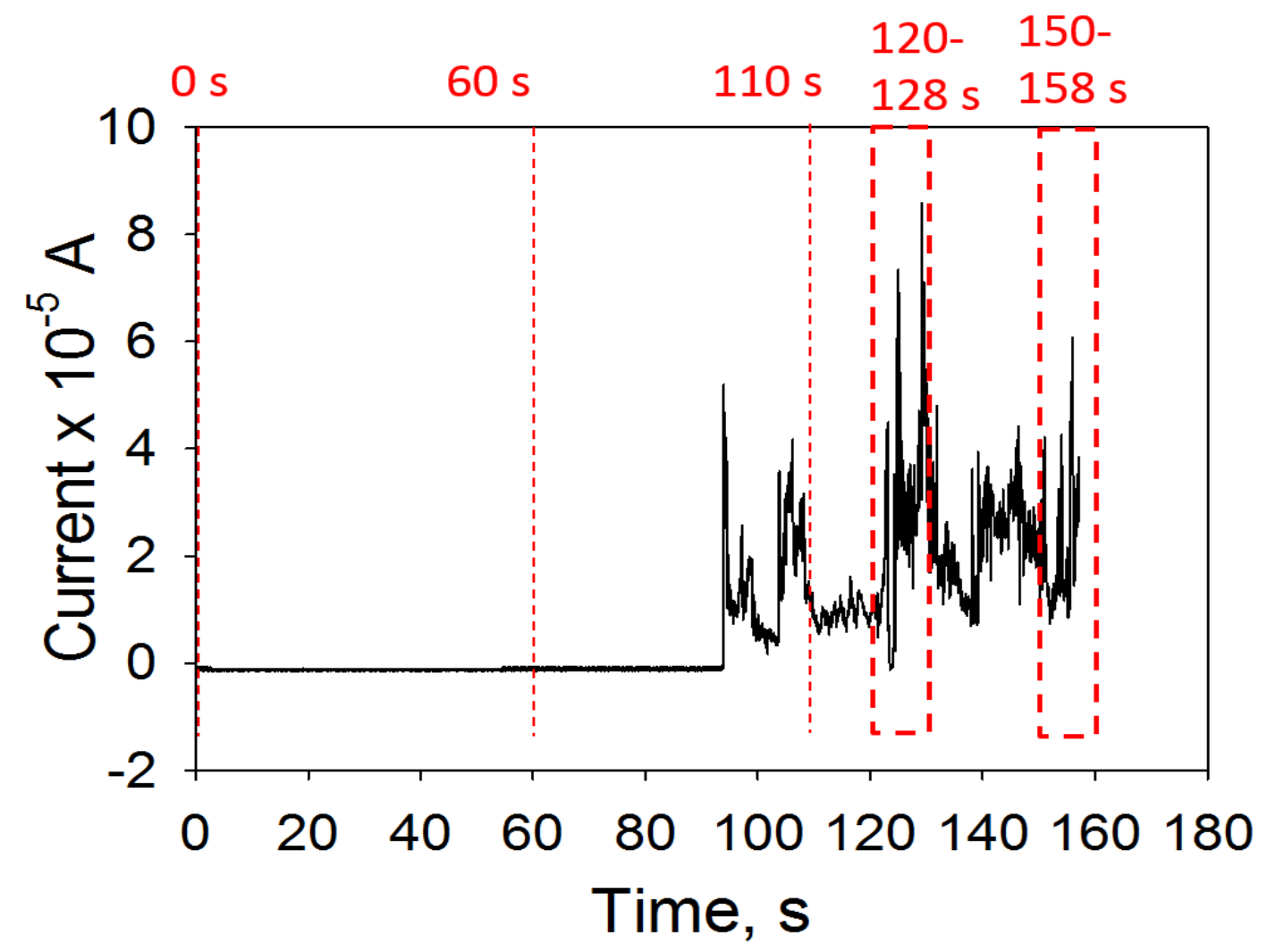

Figure 5.9. Current-time plot corresponding to the dark-field images shown in Figure 5.8. A small increase in current occurs near $60 \mathrm{~s}$ and a larger one near $95 \mathrm{~s}$, which is about $25 \mathrm{~s}$ before the appearance of the first Ag NP chain, which shows another jump in current. The appearance of a second chain occurred with another, but smaller increase in current. The current is dynamic and spiky. 
indicated by loss of Ag from the positive side, which is also consistent with loss of Ag from the positive electrode observed in SEM images.

Figure 5.10 shows two Au/dithiol/Au NPs/Ag devices after application of $0.5 \mathrm{~V}$, where one device increased in current to the $\mathrm{mA}$ level in about $100 \mathrm{~s}$ and another device increased to the $\mathrm{mA}$ level in about $450 \mathrm{~s}$. This low voltage was puzzling considering the other devices usually required $5 \mathrm{~V}$ to $10 \mathrm{~V}$ for the current to increase. The SEM images of the device in Figure 5.10C provided the explanation. The electrodeposition of Ag resulted in Ag dendrites emerging from one of the electrodes in these devices, leading to an effective electrode gap of only about $500 \mathrm{~nm}$. Since the gap was about 10 times smaller in this area, the voltage required to form Ag NP chains was also about 10 times smaller. This shows that the voltage required to form Ag NP chains is proportional to the electrode gap size.

\subsubsection{Control experiments critical to Ag NP chain formation}

Control experiments conducted with $\mathrm{Ag}$ deposited on $\mathrm{Au}, \mathrm{Au} /$ dithiol, and $\mathrm{Au} / \mathrm{dithiol} / \mathrm{Au}$ NPs (one or two sides) in the absence of CTAB showed no Ag NP chain formation at all up to $10 \mathrm{~V}$ for over $1000 \mathrm{~s}$ as shown in the Figure 5.11. This shows that CTAB is critical to Ag NP chain formation, which we believed was due to adsorbed $\mathrm{CTAB}$ on the surface. To further support this, we prepared two devices with electrodeposited $\mathrm{Ag}$ in the presence of $\mathrm{CTAB}$ onto the $\mathrm{Au} / \mathrm{dithiol} / \mathrm{Au}$ NPs IDA (one side only) and then soaked one device in water for 2 hours and treated the other device with ozone for 15 minutes. If the samples contained 

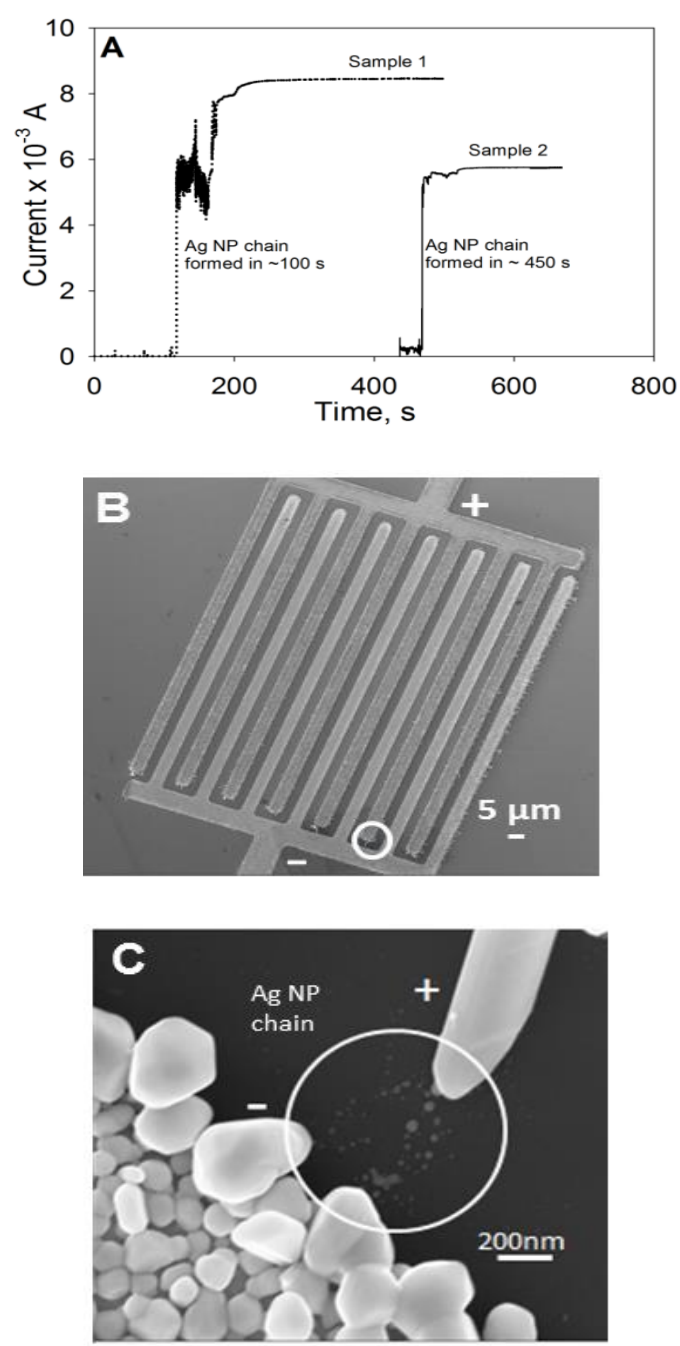

Figure 5.10. (A) Current-time plot for two Au/dithiol/Au NPs/Ag devices at $0.5 \mathrm{~V}$ showing Ag NP chain formation at this low voltage between 100s - 400s. (B) SEM image of the device whose current increased after $100 \mathrm{~s}$ in (A). (C) a zoomed-in image of the circled part of (B) showing Ag NP chain formation across a much smaller distance, which explains the low voltage required for Ag NP chain formation in this case. 

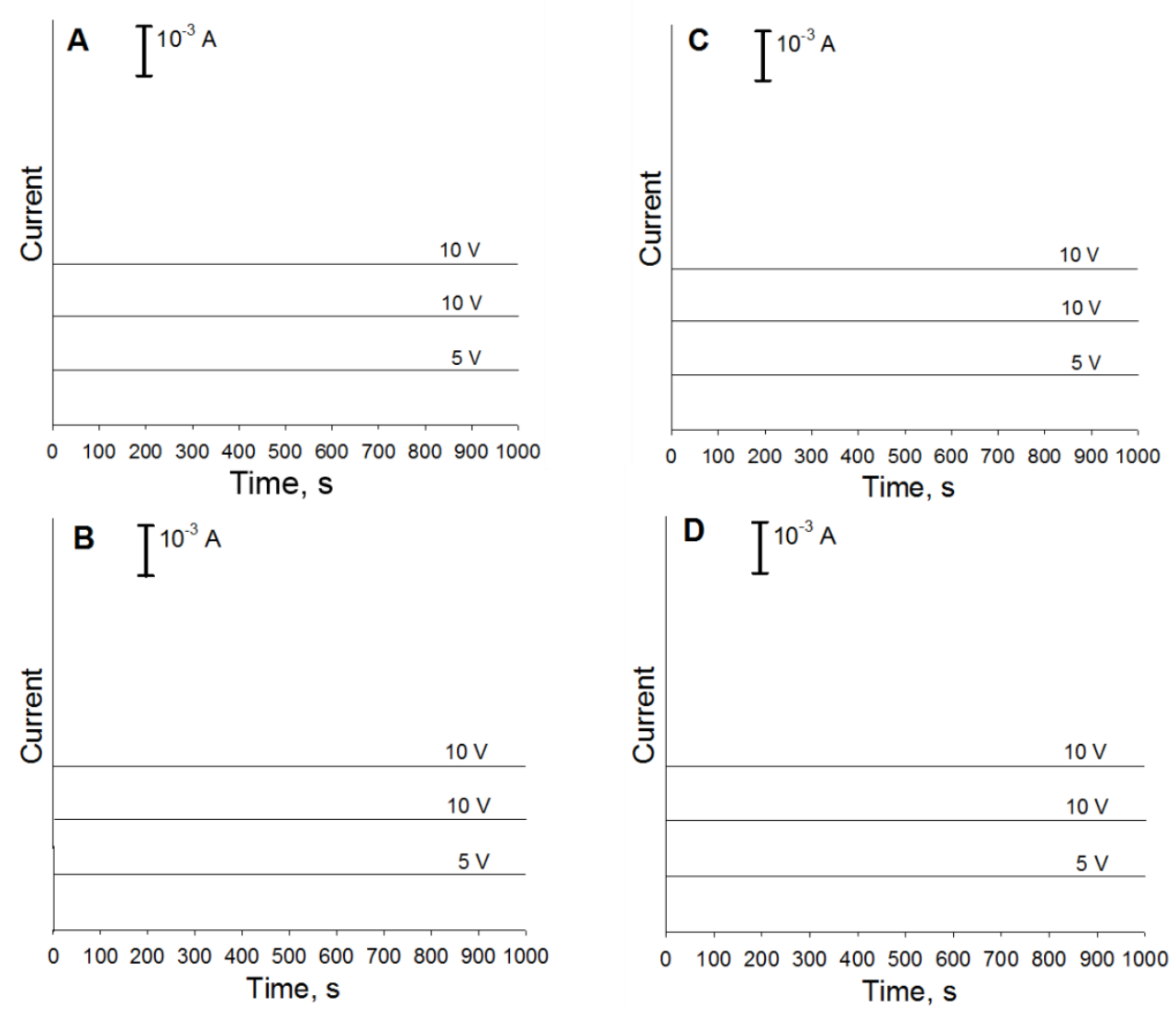

Figure 5.11. Current time plots at $5 \mathrm{~V}$ and $10 \mathrm{~V}$ as indicated from $0 \mathrm{~s}$ to $1000 \mathrm{~s}$ for the device after Ag deposition on (A) bare Au, (B) Au/dithiol, (C) Au/dithiol/Au NPs on both sides and (D) Au/dithiol/Au NPs on one side in the absence of CTAB. No current jump occurred when deposition occurred in the absence of CTAB, indicating no Ag NP chain formation. 
adsorbed $\mathrm{CTAB}$, which is critical to Ag NP chain formation, then soaking and ozone treatments would likely remove CTAB and we would not observe chain formation in the 0 to $10 \mathrm{~V}$ range ( $\mathrm{Ag}$ side positive). These control experiments did not show any Ag NP chain formation at all up to $10 \mathrm{~V}$ for over $1000 \mathrm{~s}$ as shown in the Figure 5.12, further supporting that adsorbed CTAB is important for Ag NP chain formation.

\subsubsection{Role of humidity on Ag NP chain formation}

Figure 5.13 shows the I-t plot of an Au/dithiol/Au NPs device with Ag deposited on it in the presence of CTAB. Application of $10 \mathrm{~V}$ (Ag side positive) for over $1000 \mathrm{~s}$ under dry $\mathrm{N}_{2}$ led to background $\mathrm{pA}$ level current, indicating no $\mathrm{Ag}$ NP chain formation when under dry $\mathrm{N}_{2}$. Once the same sample was introduced to humid air later, the current increased to the $\mathrm{mA}$ level within $150 \mathrm{~s}$ at $5 \mathrm{~V}$, consistent with Ag NP chain formation. This shows that both adsorbed CTAB and humidity are required for Ag NP chain formation. We carried out experiments to observe the chain formation at different humidity level from $20 \%$ to $50 \%$. The experimental set up is described in Chapter II experimental section. Figure 5.14 shows the current time plots for Ag NP chain formation on Au/dithiol/Au NP/Ag IDA or TED device in presence of CTAB with Ag deposited on one side of Au dithiol. We found that at humidity level $20 \%$, we could not observe any high current up to $10 \mathrm{~V}$ for $1000 \mathrm{~s}$. As the humidity level increased, we could observe the chain formation starting from $25 \%$ on applying $10 \mathrm{~V}$. The voltage to form chain formation decreased upon increasing the percentage level of humidity. 


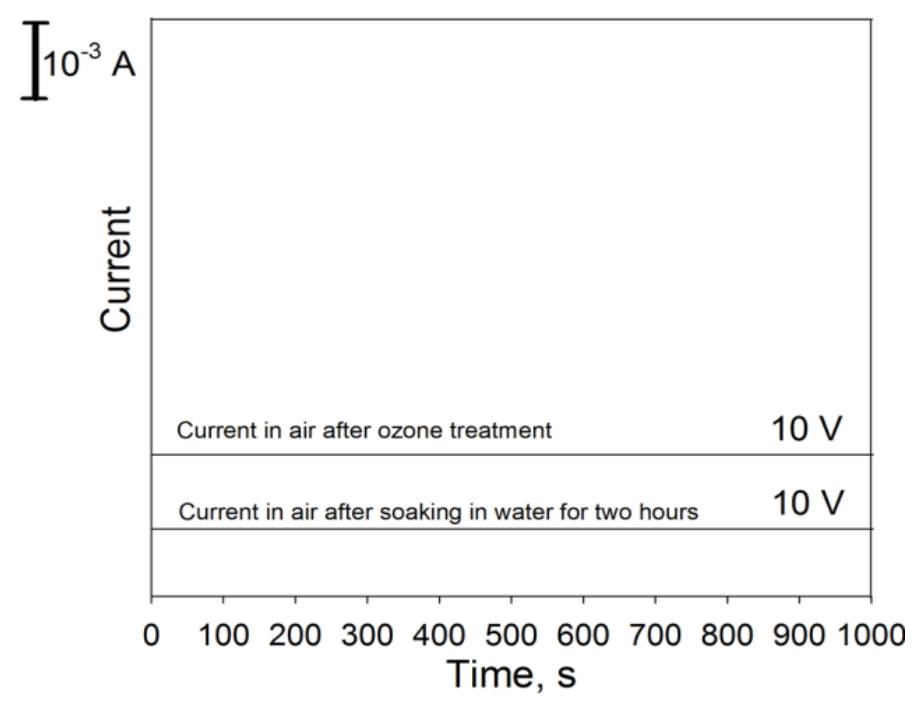

Figure 5.12. Current-time plots of an electrode device with Au/dithiol/Au NPs/Ag on one side of the device prepared by Ag electrodeposition in the presence of CTAB. Ozone treatment or soaking in water for $2 \mathrm{~h}$ prior to the application of 10 $\mathrm{V}$ (Ag side positive) prevents Ag NP chain formation from occurring. This is likely due to the removal of adsorbed CTAB from the surface, which is necessary for Ag NP chain formation. 


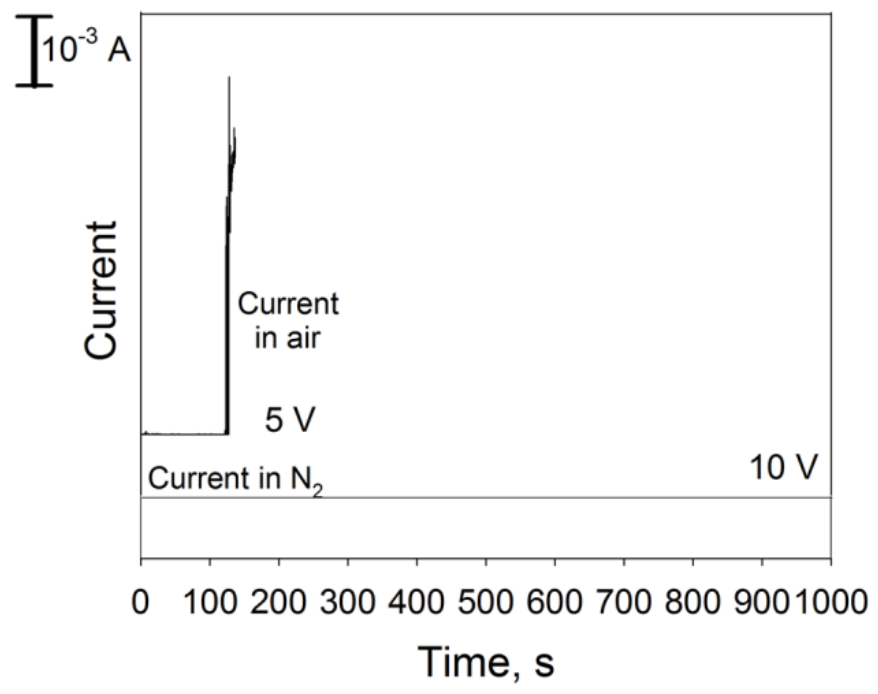

Figure 5.13. Current-time plots obtained at the indicated voltages on IDA device with Ag deposited in the presence of CTAB on one side of a Au/dithiol/Au NPs device. The current remained at the instrument background level up to $10 \mathrm{~V}$ ( $\mathrm{Ag}$ side positive) when in the presence of dry $\mathrm{N}_{2}$. The current increased to the $\mathrm{mA}$ level due to Ag NP chain formation once the same device was later introduced to humid air. This shows that humidity is crucial for Ag NP chain formation. 

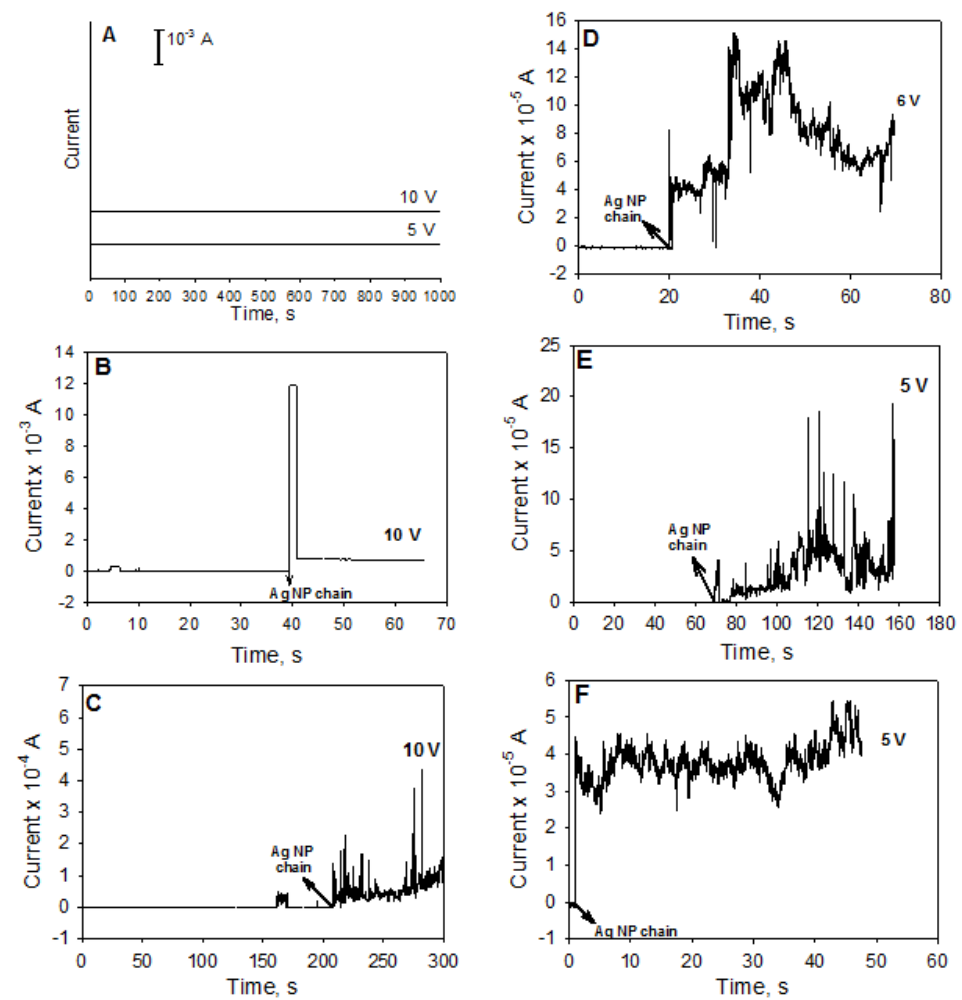

Figure 5.14. I-t plots obtained at the indicated voltages on IDA or TED devices with Ag deposited in the presence of CTAB on one side of the device with Au NP and thiol for different humidity level. (A) $20 \%$, current remained at pA level upto $10 \mathrm{~V}$ for $1000 \mathrm{~s}$, (B) $25 \%$, rise in current was observed at $10 \mathrm{~V}, \sim 40 \mathrm{~s}$, (C) $30 \%$, rise in current was observed at $10 \mathrm{~V}, \sim 210 \mathrm{~s}$, (D) $35 \%$, rise in current observed at $6 \mathrm{~V}, \sim 20 \mathrm{~s},(\mathrm{E}) 40 \%$, rise in current observed at $5 \mathrm{~V}, \sim 70 \mathrm{~s}$ and (F) 50\%, rise in current observed at $5 \mathrm{~V}, \sim 2 \mathrm{~s}$. The rise in current for the devices indicated Ag NP chain formation. 
Figure 5.15 shows SEM images of the different IDA or TED devices after Ag deposition in the presence of CTAB and application of $5 \mathrm{~V}$ or $10 \mathrm{~V}$. The SEM images are consistent with the current time plots in Figure 5.14. Table 5.1 summarizes the Figures 5.14 and 5.15. From this table we can say that the voltage required for the formation of chains increases with decreasing humidity and chains do not form at all up to $10 \mathrm{~V}$ at a humidity of $20 \%$ or lower.

\subsubsection{Determination of mechanism for the formation of Ag NP chains}

Since Ag NP chain formation required humidity and CTAB and the chains formed by movement of $\mathrm{Ag}$ from the positive electrode to the negative electrode, we believed that the formation mechanism could be due to either electromigration of positively-charged $\mathrm{CTA}^{+}$-coated Ag NPs from the positive to the negative electrode and reduction at the negative electrode, where CTAB acts as supporting electrolyte. Both mechanisms could be facilitated by a thin layer of water adsorbed on the device in humid air, depend strongly on adsorbed CTAB, and result in movement of $\mathrm{Ag}$ from the positive to negative electrode. The electrochemical mechanism has been described previously in the formation of $\mathrm{Ag}$ filaments in resistive switching devices. ${ }^{103,110,116,193,194}$ To distinguish between these two possibilities, we post-treated Ag-coated Au IDA devices with CTAB, negatively-charged surfactant sodium dodecyl sulfate (SDS), and negativelycharged citrate ions before applying various voltages in air to determine if Ag NP chain formation would occur in these cases. 

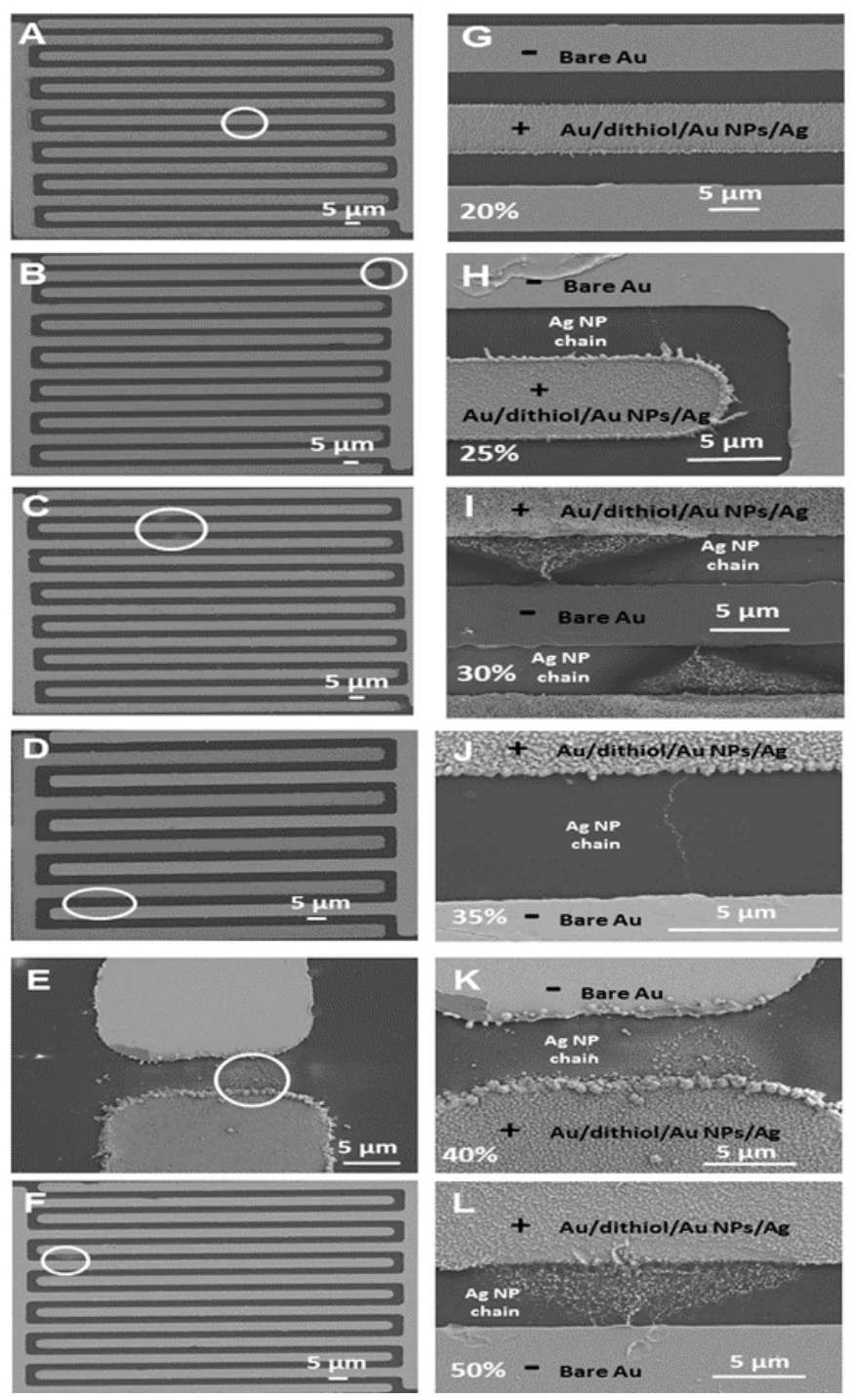

Figure 5.15. SEM images of IDA or TED devices after Ag deposition in presence of CTAB and application of voltage for various times and formation of chains at different voltages depending upon the percentage level of humidity. (A, G) 20\% humidity level does not show any chains, which is consistent with the current-time plot shown in Figure 5.14A. (B-E) Ag NP chains are formed on IDA or TED devices. We observed that as the humidity level increases, voltage at which chains form decreases. (H-L) Zoomed in images of the circled parts in (B-E). 
Table 5.1. Summary of the humidity experiment showing humidity and voltage required to form chains at different $\%$ of humidity.

\begin{tabular}{|c|c|}
\hline Relative Humidity & Voltage required to form chains \\
\hline $20 \%$ & $\begin{array}{c}\text { No chain formed upto } 10 \mathrm{~V} \text { for } \\
1000 \mathrm{~s}\end{array}$ \\
\hline $25 \%$ & $10 \mathrm{~V}$ \\
\hline $30 \%$ & $10 \mathrm{~V}$ \\
\hline $35 \%$ & $6 \mathrm{~V}$ \\
\hline $40 \%$ & $5 \mathrm{~V}$ \\
\hline $50 \%$ & $5 \mathrm{~V}$ \\
\hline
\end{tabular}


Soaking an $\mathrm{Au} / \mathrm{Ag}$ device ( $\mathrm{Ag}$ on both sides) in $0.1 \mathrm{M} \mathrm{CTAB}$ for 1 hour, rinsing with water, and drying under nitrogen or soaking in $1.0 \times 10^{-4} \mathrm{M} \mathrm{CTAB}$ for 1 hour and allowing to air dry without rinsing resulted in Ag NP chain formation following a $10 \mathrm{~V}$ and $5 \mathrm{~V}$ application, respectively. No Ag NP chains formed with no exposure to CTAB as shown in Figure 5.16. As observed when the Ag was deposited in the presence of $\mathrm{CTAB}$, movement of $\mathrm{Ag}$ was from the positive to negative electrodes and chain - like structures formed as shown in Figure 5.17. Soaking an $\mathrm{Au} / \mathrm{Ag}$ device in $0.1 \mathrm{M}$ SDS for 1 hour, rinsing with water, and drying under nitrogen or soaking in aqueous $1 \times 10^{-4} \mathrm{M}$ SDS for 1 hour and allowing it to air dry without rinsing also resulted in Ag NP chain formation, but following a $4 \mathrm{~V}$ or $3 \mathrm{~V}$ application, respectively as shown in the Figure 5.18. The movement of Ag was still from the positive to negative electrodes. Soaking an Au/Ag device in $1 \mathrm{x}$ 10-4 M citrate solution for 1 hour and allowing it to air dry without rinsing also resulted in Ag connections across the gap at $3 \mathrm{~V}$ shown in Figure 5.19. From SEM images, we found that instead of well-defined Ag NP chains, larger Ag aggregate structures connected the gap, again by movement of $\mathrm{Ag}$ from the positive to negative electrode. 


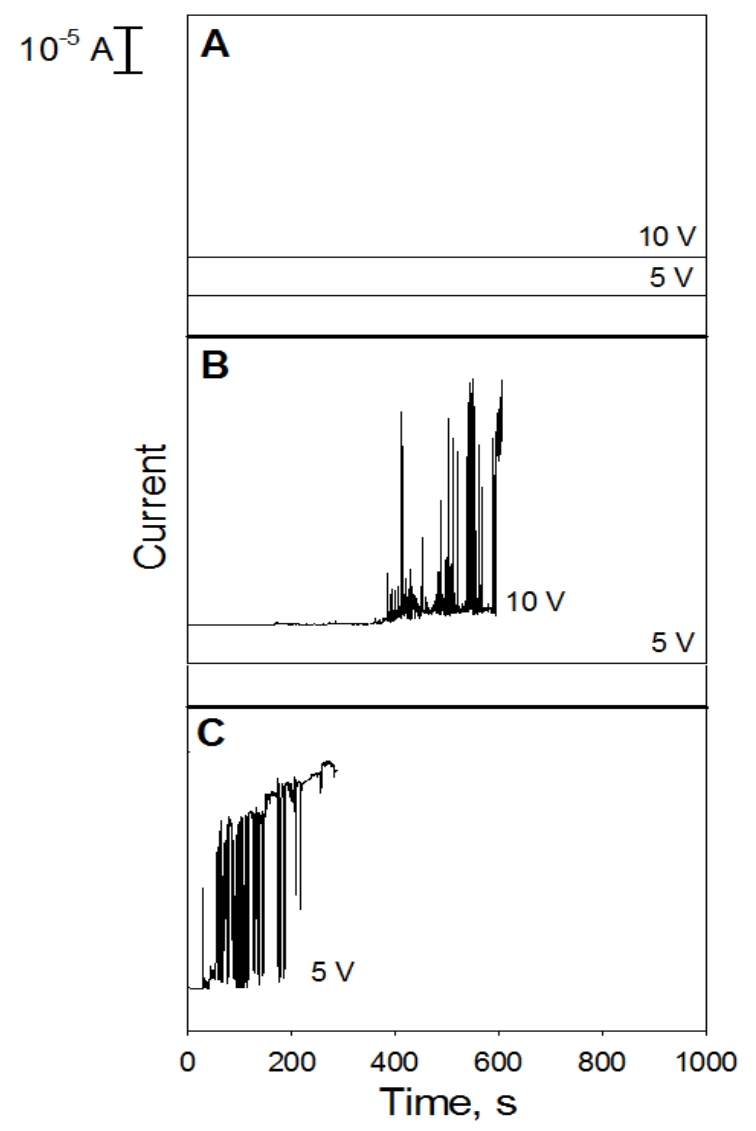

Figure 5.16. Current-time plots at 5 or $10 \mathrm{~V}$ as indicated of IDA devices coated with $\mathrm{Ag}$ on both sides and (A) not treated further, (B) soaked in aqueous $0.1 \mathrm{M}$ CTAB for 1 hour, rinsed with water, and dried in $\mathrm{N}_{2}$, and (C) soaked in aqueous 1 $\times 10^{-4} \mathrm{M} \mathrm{CTAB}$ solution for 1 hour, not rinsed, and dried in air for 2 hours. This shows that Ag NP chains can form from devices post-treated with CTAB, not just those with $\mathrm{Ag}$ deposited in the presence of CTAB. It also shows that the voltage for Ag NP chain formation decreases as the amount of CTAB increases. This likely explains why 2 -sided devices required lower voltage than one-sided devices. 

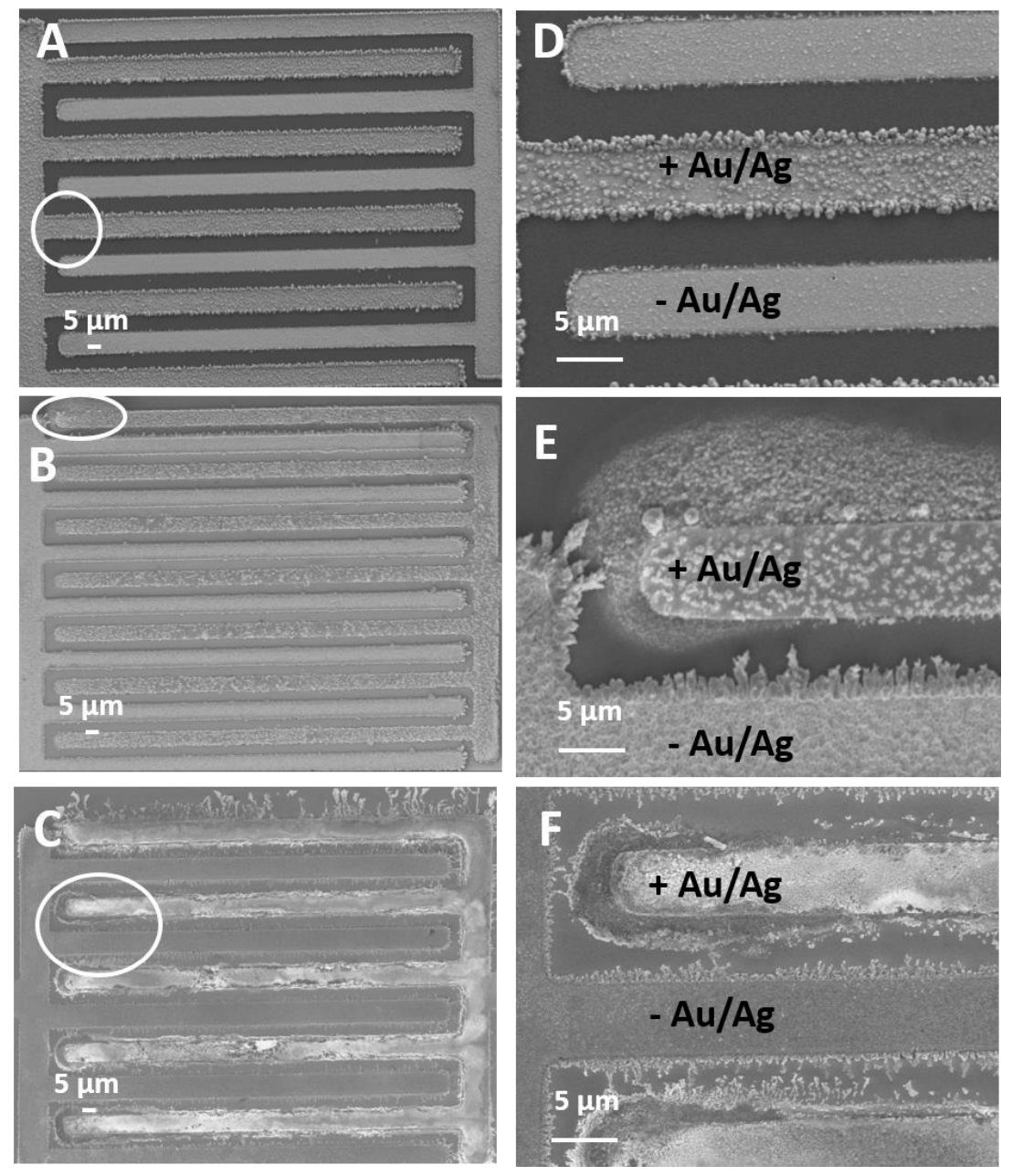

Figure 5.17. SEM images of $\mathrm{Au} / \mathrm{Ag}$ devices $(\mathrm{A}, \mathrm{D})$ untreated, $(\mathrm{B}, \mathrm{E})$ treated with $0.1 \mathrm{M} \mathrm{CTAB}$ and rinsed, and $(\mathrm{C}, \mathrm{F})$ treated with $1 \times 10^{-4} \mathrm{M}$ CTAB but not rinsed, corresponding to the I-t plots A, B, and C in Figure 5.16, respectively. Post treatment with CTAB leads to Ag chain-like structures caused by Ag movement from the positive to negative electrode, similar to devices with Ag deposited in the presence of CTAB. 

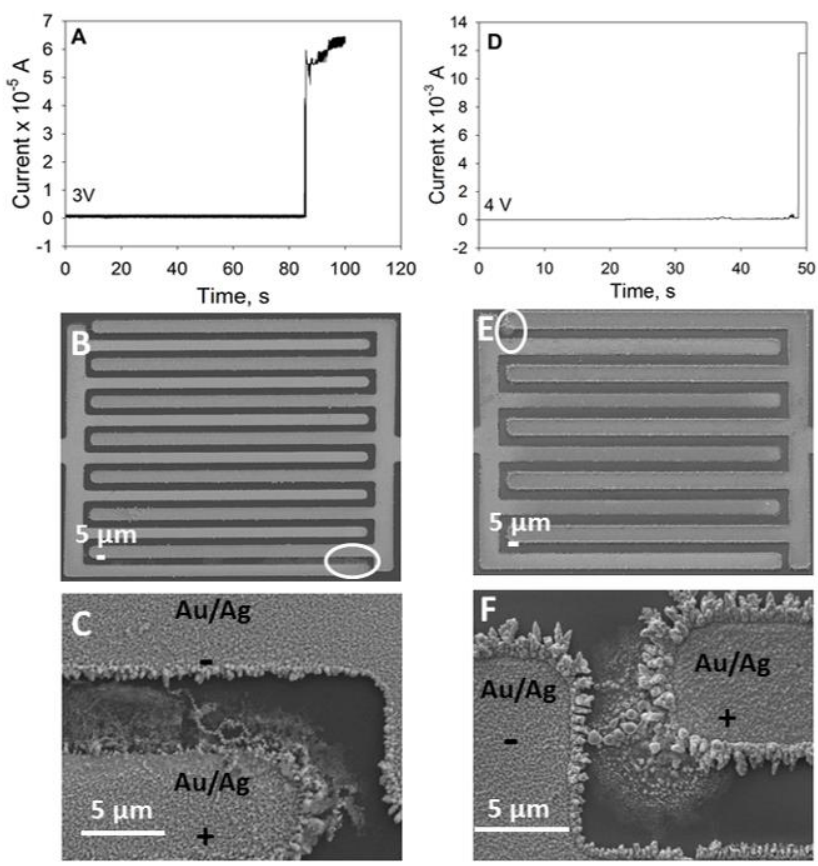

Figure 5.18 (A) Current-time plot of an $\mathrm{Au} / \mathrm{Ag}$ (two sides) device soaked in $1 \mathrm{x}$ 10-4 M SDS and air dried for 2 hours. (B, C) SEM images of the device after the current-time plot in (A). (D) Current-time plot of an $\mathrm{Au} / \mathrm{Ag}$ device soaked in aqueous 0.1 M SDS for 1 hour, rinsed with water and dried in $\mathrm{N}_{2}$. (E, F) SEM images of the device after the current-time plot in (D). The current increased at 3 $\mathrm{V}$ after $\sim 85 \mathrm{~s}$ or $4 \mathrm{~V}$ after $45 \mathrm{~s}$ due to $\mathrm{Ag}$ chain-like structures growing across the gap. The Ag moved from the positive to negative electrode in the same way as when positively-charged CTAB was used. This suggests an electrochemical mechanism is responsible for the Ag NP chain formation. 

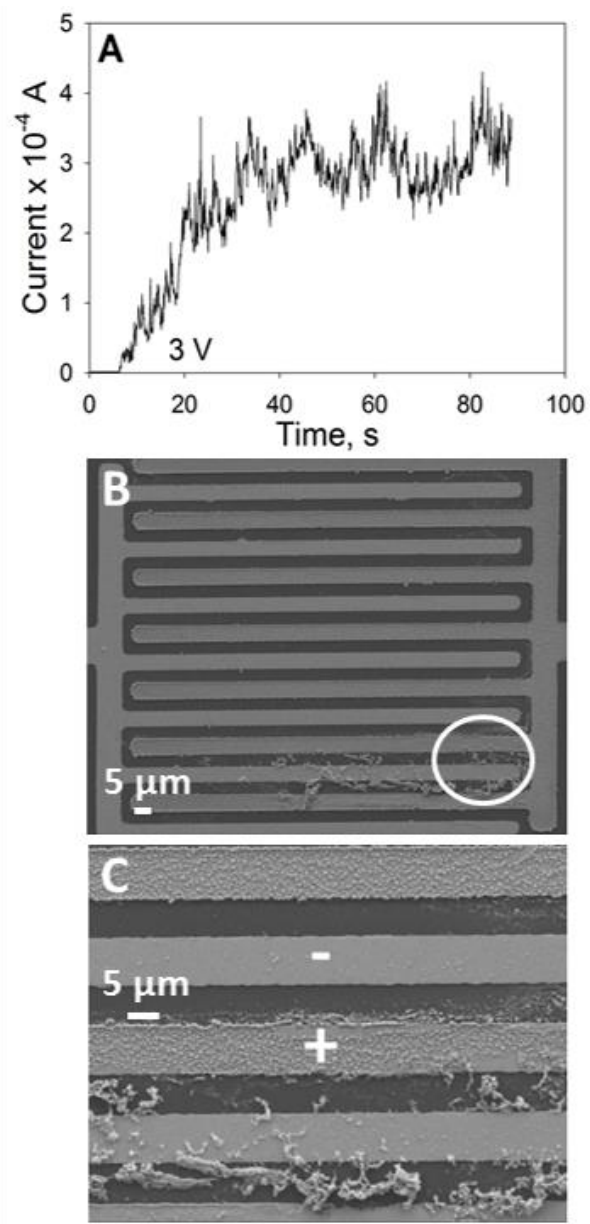

Figure 5.19. (A) Current-time plot of an $\mathrm{Au} / \mathrm{Ag}$ device (Ag on one side) soaked in 1x10-4 M citrate solution and air dried for 2 hours. $(B, C)$ SEM images of the device after the current-time plot in (A). The current increased at $3 \mathrm{~V}$ after $5 \mathrm{~s}$ due to Ag migration from the positive to negative electrode. The Ag got removed from some parts of the electrode and crossed the other side. 


\subsubsection{Explanation of Scheme of Electrochemical Mechanism for Ag NP chain formation}

For all of the devices soaked or coated with CTAB, SDS, or citrate, we found that the device showed oxidation and reduction peaks when scanning from $-1 \mathrm{~V}$ to $+1 \mathrm{~V}$ in air as shown in Figure 5.20. The peaks were more prominent for CTAB and SDS. The pair of peaks shows clear electrochemical processes occurring on these devices in air. Also, whether the surfactant or ions are positively-charged or negatively-charged, the Ag NP chains always formed by movement of Ag from the positive to the negative electrode. We therefore conclude that the Ag NP chain formation is an electrochemical process with the CTAB, SDS, or citrate ions merely acting as supporting electrolyte in a humid environment to facilitate the electrochemical process. This rule out the possibility that $\mathrm{CTA}^{+}$ions make the $\mathrm{Ag}$ NPs positively-charged so that they move by electromigration from the positive to negative electrode. If that was true, then SDS and citrate would make the Ag NPs negatively -charged and they would move by electromigration from the negative to the positive electrode, which did not occur. Our data are therefore more consistent with an electrochemical mechanism involving oxidation of $\mathrm{Ag}$ to $\mathrm{Ag}^{+}$at the positive electrode and re-deposition of Ag on the negative electrode by reduction of $\mathrm{Ag}^{+}$. The voltage required to form the $\mathrm{Ag} \mathrm{NP}$ chains depends on the electrode gap, but also the amount of surfactant, which explains the variation from $0.5 \mathrm{~V}$ for short gaps to $10 \mathrm{~V}$ for the larger gaps and the different voltage for one sided versus two sided devices and different electrode treatment. These all likely lead to 

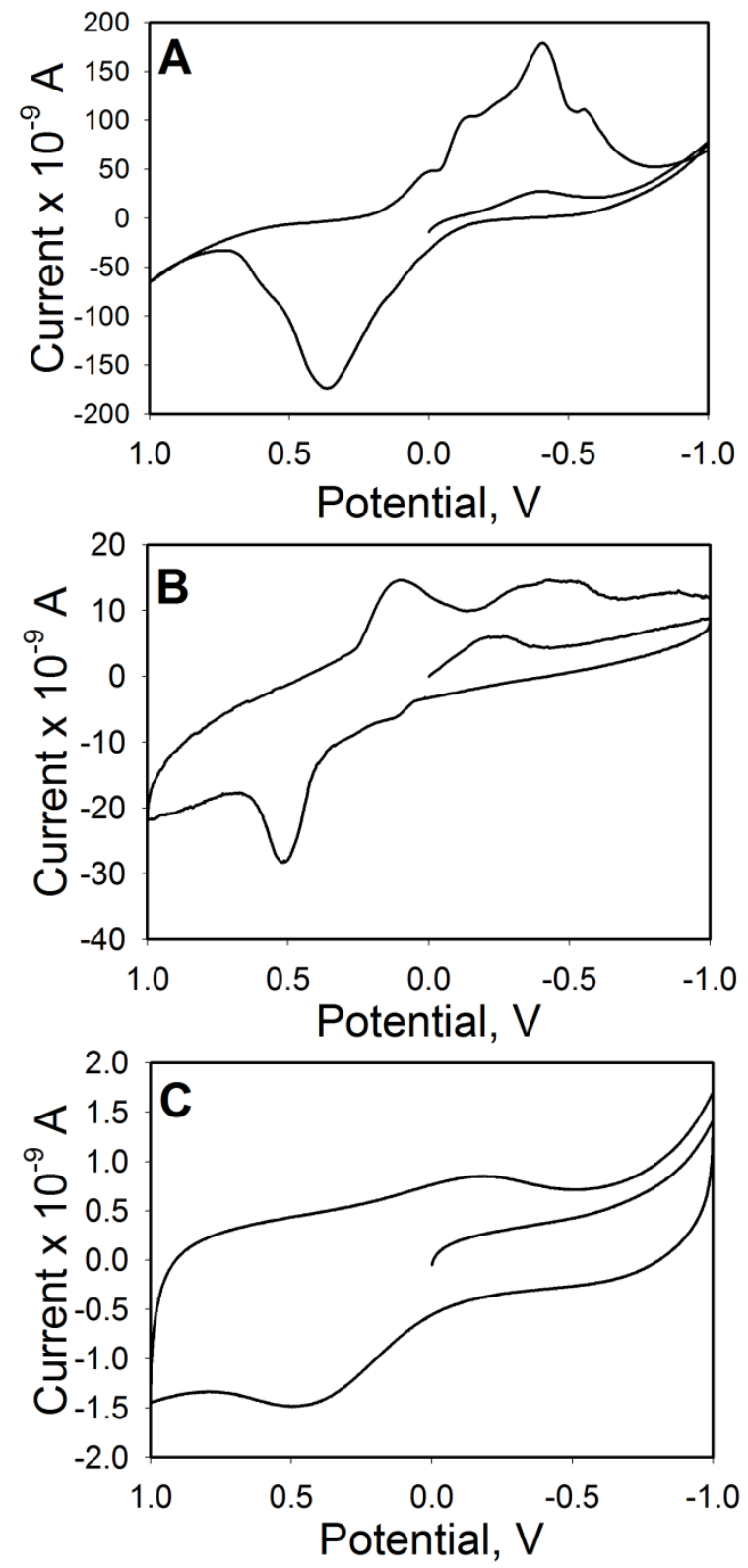

Figure 5.20. $\mathrm{I}-\mathrm{V}$ plots of $\mathrm{Au} / \mathrm{Ag}$ devices after it was soaked in aqueous $1 \times 10^{-4} \mathrm{M}$ electrolyte solution of (A) CTAB, (B) SDS, and (C) Citrate for 1 hour and allowed to air dry without rinsing for 2 hours 
different amounts of CTAB on the surface. Increased amount of surfactant and shorter gaps lead to lower resistance to facilitate the electrochemical process.

Scheme 5.1 illustrates the electrochemical mechanism that we believe leads to Ag NP chain formation. Ag NPs with adsorbed CTAB or other ions are electrochemically oxidized at the positive terminal under the applied potential and then become reduced and redeposit at the negative terminal, eventually connecting the two electrodes in the form of a chain. The growth process of the nanoparticle chains is very similar to the formation of Ag filaments described for resistive switching in other solid electrolyte systems. ${ }^{99,123,131,195}$ Those studies also observed a cylindrical or cone-shaped Ag NP chain feature at the positive electrode and a narrower linear region of the chain at the negative electrode.

\subsection{Conclusions}

We observed Ag NP chain formation driven by a voltage between electrode gaps separated by as large as a $5 \mu \mathrm{m}$ distance. We tested bare $\mathrm{Au}, \mathrm{Au} / \mathrm{dithiol}$, and $\mathrm{Au} / \mathrm{dithiol} / \mathrm{Au}$ NPs (one or two sided) that had $3.0 \pm 0.3 \times 10^{-5} \mathrm{C}$ of $\mathrm{Ag}$ electrodeposited on them in the presence of $\mathrm{CTAB}$ or that were coated with $\mathrm{Ag}$ and untreated or post treated with CTAB, SDS, or citrate by soaking or dropcoating. After a voltage was applied in humid air, Ag NP chains formed across the microgap due to electrochemical oxidation of $\mathrm{Ag}_{\mathrm{g}} \mathrm{Ag}^{+}$at the positive electrode and re-deposition of $\mathrm{Ag}^{+}$to $\mathrm{Ag}$ at the negative electrode. The surfactant acted as a supporting electrolyte and the humidity provided a thin water layer on the device 

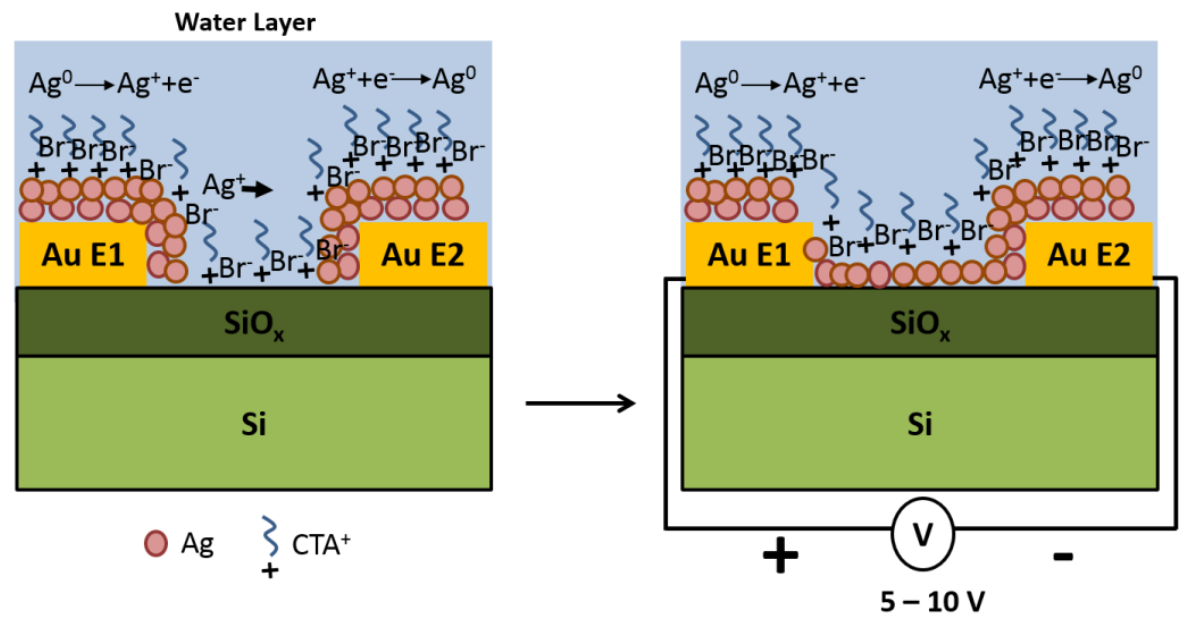

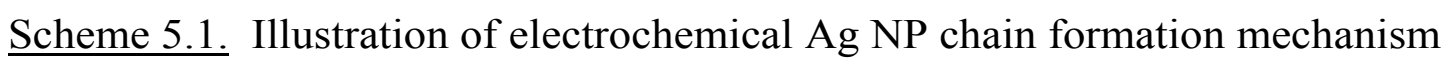
between the two Au electrodes. 
to complete the electrochemical cell. No Ag NP chains formed in the absence of these critical components. The voltage required for Ag NP chain formation depended on the amount of surfactant and electrode gap, which varied depending on the electrode treatment method or surfactant treatment method. This work importantly provides a new method for forming Ag NP chains across micron-sized electrode gaps in air. Not only can this approach be used to fabricate interesting plasmonic devices, SERS substrates, nanoscale chemi-resistors, or resistive switches, it is a useful approach for better understanding the electrochemical formation of metal chain and filament structures in electrochemical systems. 


\section{CHAPTER VI}

\section{RESISTIVE SWITCHING BEHAVIOR OF ELECTROCHEMICALLY SYNTHESIZED ONE-DIMENSIONAL SILVER NANOPARTICLE CHAINS}

\subsection{Introduction}

In recent years a notable progress in the miniaturization of electronic devices has been achieved in which the main component that has shown great interest is electronic memory. All computers require storage space and memory to run programs and store files. There are different types of memory and storage, each with its own purpose. The external storage devices are used to store additional storage other than that in the computer. They are classified as optical storage, magnetic storage and solid state storage devices. Optical storage involves CDs and DVDs; magnetic storage involves floppy discs, and solid state storage involves flash memory.

There are two types of flash memory: NAND (Not AND, binary operation in logic) and NOR (Not OR, binary operation in logic) type. NAND Flash allows for a high storage capacity and supports a fast writing/erasing rate and therefore has been widely used for storage applications including memory cards, USB drives, and solid state drives. ${ }^{196}$ Compared with NAND Flash, NOR Flash usually gives a faster readout rate but with a disadvantage of expensive storage density. Hence, NOR Flash is often used where it finds applications in almost all handheld devices 
including cellphones and PDAs. Despite the rapid growth, both NOR and NAND flash memories face technology challenges when further scaling down to less than $20 \mathrm{~nm} \cdot{ }^{197-199}$ Flash memory, which is currently very popular, has very high capacity and is nonvolatile, but is relatively slow and approaching its fundamental limits, more advanced storage nanodevices are being explored. ${ }^{107,198}$ A review article describes the importance of emerging nonvolatile memories and recent focus on turning rigid silicon-based memory technology into a soft nonvolatile memory technology. ${ }^{200}$ Emerging nonvolatile memories, such as ferroelectric random access memory (FRAM), magnetic random access memory (MRAM), phase-change random access memory (PCRAM), and resistive random access memory (RRAM) have been extensively studied due to their fast write speed, low power consumption, longevity, and good scalability. ${ }^{201}$ Among these candidates, RRAM is considered to be the most promising as it operates faster than PCRAM and it has a simpler and smaller cell structure than magnetic memories (e.g., MRAM or STT-RAM) as described by Xu et al. ${ }^{202}$

Resistive switching (RS) memory is expected to be the next generation memory device because of its high density, low power consumption, and high speed. ${ }^{203-205}$ Resistive switching involves a change in resistance of a material by applying an external voltage and the device maintaining that resistance when the voltage is no longer applied. The basic characteristic of Resistive Random Access Memory (RRAM) is the two different resistive states. The operation which changes the resistance of the device from the high resistance state (HRS) to low 
resistance state (LRS) is called a 'SET' process, while the opposite process is defined as 'RESET'. The switching is referred to as unipolar when the change of resistance does not depend on the polarity of the applied voltage and bipolar when it does. ${ }^{206}$ Several switching mechanisms have been described in the literature, including those based on conducting filaments $(\mathrm{CFs}),{ }^{207-210}$ charge trapping/detrapping, ${ }^{211}$ oxygen ion migration, ${ }^{212}$ and interfacial barrier regulation. ${ }^{213}$ Ion migration, ${ }^{214,215}$ where the switching process originates from the motion of charged particles driven by an electric field, ${ }^{216}$ is categorized into two types. Cation migration (e.g. $\mathrm{Ag}^{+}$or $\mathrm{Cu}^{2+}$ ions) occurs in some chalcogenides $(\mathrm{AgS}$ and $\mathrm{CuS}),{ }^{217}$ oxides $\left(\mathrm{TiO}_{2}, \mathrm{ZrO}_{2}\right),{ }^{3,218,219}$ and amorphous $\mathrm{Si}^{220,221}$ Anion migration ${ }^{222,223}$ involves compounds like $\mathrm{TiO}_{2}$, where $\mathrm{O}^{2-}$ migrates. Charge trapping/detrapping occurs with irregular positive traps in doped materials.

Resistive random access memory (RRAM) based on CFs stands out for its excellent performance. ${ }^{24,225}$ One type of CF device comprises a dielectric layer sandwiched between an electrochemically active electrode (Ag for example) and an inert electrode metal (Pt for example). Metal filament growth occurs by cationic transport in the dielectric film. When a positive voltage is applied on the active $\mathrm{Ag}$ electrode, $\mathrm{Ag}^{+}$ions are generated and migrate towards the inert $\mathrm{Pt}$ cathode, where $\mathrm{Ag}^{+}$becomes reduced to $\mathrm{Ag}^{0}$. $\mathrm{CFs}$ of $\mathrm{Ag}$ grow from the cathode towards the anode until a connection forms, leading to low resistance. Reversing the process leads to a break in the $\mathrm{Ag} \mathrm{CF}$ and high resistance. Other mechanisms of CFs involve joule heating, ${ }^{226,227}$ electrochemical processes in binary oxides, ${ }^{228}$ 
redox processes in ionic conductors such as oxides, chalcogenides and polymers, ${ }^{216,229}$ field-assisted drift/diffusion of ions in $\mathrm{TiO}_{2}$ based memristors, ${ }^{230,222}$ atomic migration ${ }^{118}$ and amorphous-silicon based resistive switches. $^{231,232}$

Ag based CF devices are based on metallic filament formation/fracture, where formation of the metallic Ag path is responsible for the decrease in resistance and breaking of the path leads to high resistance. Ag based non-volatile resistive memory devices are fabricated because $\mathrm{Ag}$ is abundant and it is a low cost optimal electrode material. Several reports have appeared based on the method of controlling transport of silver ions in mixed-conductor silver sulfide $\left(\mathrm{Ag}_{2} \mathrm{~S}\right)$ crystals. ${ }^{231,233-239}$ These devices show unique Ag atom deposition and resistance switching. The switches operate through the formation and dissolution of an atomic bridge between the electrodes and the behavior is realized by control of the local solid-state electrochemical reaction. Using the solid-state electrochemical phenomenon, it is possible to control the ion transport locally on the surface or at a hetero-interface composed of such materials. From conductivity measurements, Ag NP chains exhibit nonlinear, rather than ohmic, electrical properties. ${ }^{79}$

Our group has recently focused on Pd and Ag nanowire (NW) junctions for sensing, molecular electronics, and surface-enhanced Raman spectroscopy (SERS) applications. ${ }^{37,38,240}$ In our attempt to synthesize more controlled Ag NW structures, we discovered a new approach to form Ag NP chains across $5 \mu \mathrm{m}$ electrode gap by performing electrochemistry in humid air. Our previous work 
focused on the mechanism of forming the Ag NP one-dimensional (1D) chain assemblies. The Ag NP chains are formed by electrodeposition of Ag onto the IDA or TED devices followed by application of $5 \mathrm{~V}$ for variable times, but usually 100-500 s. After Ag NP chain formation, the devices were scanned from 0 to $5 \mathrm{~V}$ in air for a few cycles, which leads to devices with high resistance above $3 \mathrm{~V}$ (OFF state) and low resistance from 0 to $3 \mathrm{~V}$ (ON state). The resistance is not linear with voltage, providing a resistance switch with $\mathrm{ON} / \mathrm{OFF}$ ratios of 100 or greater, switching times less than $1 \mathrm{~ms}$, and endurance of at least 1000 cycles. The switching behavior does not depend on the presence of surfactant or humidity, suggesting that atomic migration at the Ag NP junctions may be involved in the $\mathrm{ON} / \mathrm{OFF}$ switching behavior. The mechanism is likely due to Ag atom migration leading to connections and breaks at the Ag NP junctions at different potentials.

\subsection{Experimental Section}

Wiring and cleaning of the electrodes. Refer to Chapter II section 2.2.3.

Electrode functionalization. Refer to Chapter II section 2.2.4.

Electrodeposition of Ag on 5 micron gap electrodes using cyclic voltammetry. Refer to Chapter II section 2.4.1.

Ag nanoparticle chain formation. Refer to Chapter II section 2.4.3.

Resistive switching of Ag nanoparticle chain formation. Refer to Chapter II section 2.4.4. 
Characterization of the device was performed by SEM as explained in Chapter II section 2.3.2.

\subsection{Results and Discussion}

We previously described the formation of one-dimensional (1D) Ag nanoparticle (NP) chains between two electrodes separated by $5 \mu \mathrm{m}$ that had Ag electrodeposited on them in the presence of cetlytrimethylammonium bromide (CTAB). ${ }^{40}$ The chains formed after applying 5-10 $\mathrm{V}$ between the electrodes in air. The formation was an electrochemical process, facilitated by the water layer formed on the surface when in humid air and the ions from CTAB as electrolyte. In this paper we describe the interesting electronic properties of these Ag NP chains.

\subsubsection{Resistive switching of IDA electrodes with Ag electrodeposited on both sides of the device}

Figure 6.1 shows the current-time plots of a Au IDA device in air that was previously functionalized with 1,8-octanedithiol and $4 \mathrm{~nm}$ diameter Au NPs before having $6 \times 10^{-5}$ Coulombs of Ag electrodeposited on each side in the presence of CTAB. The plots show no current flowing through the device while applying $1 \mathrm{~V}$ and $3 \mathrm{~V}$ for $200 \mathrm{~s}$ each. While applying $5 \mathrm{~V}$, though, the current rose about 8 orders of magnitude within $10 \mathrm{~s}$. As the current rises, there are fluctuations in the current at times that causes the current to drop to zero again, but the current eventually becomes fairly stable at the mA level after about $150 \mathrm{~s}$ for this device. 


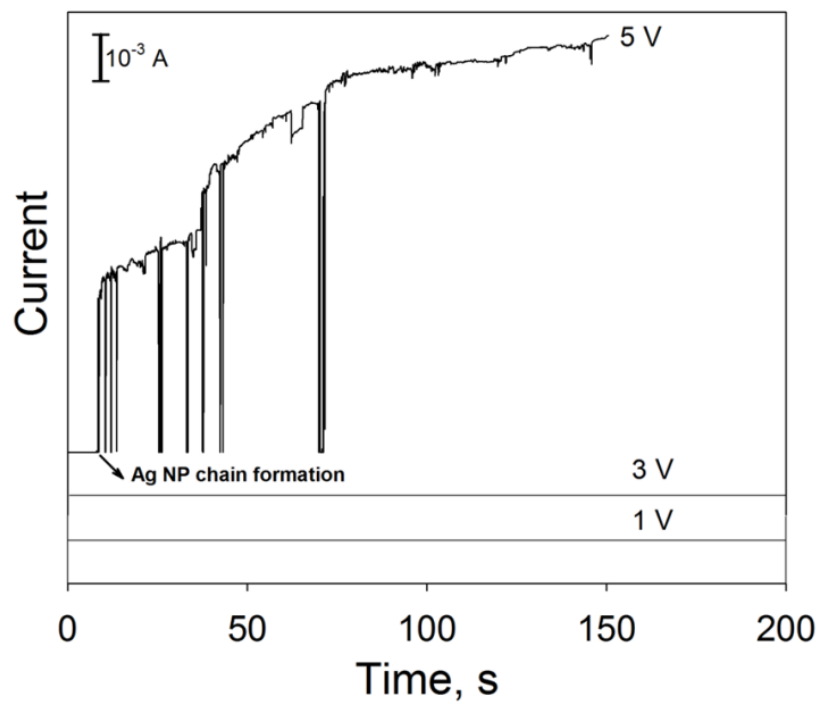

Figure 6.1 Current-time plot obtained in air at the indicated voltages on a $5 \mu \mathrm{m}$ gap Au IDA device functionalized with octanedithiol and $4 \mathrm{~nm}$ Au NPs before electrodeposition of $\mathrm{Ag}$ in the presence of $\mathrm{CTAB}$ on both sides. The increase in current with fluctuations is a characteristic of the Ag NP chain formation process across the electrode gap. 
This rise in current and fluctuations are typical for the Ag NP chain formation as we described previously. ${ }^{40}$ This process if dynamic, likely involving deposition and dissolution along the chains as they form before finally becoming more stable. After the chain formation, the device can be made to switch between a high resistance state (HRS) and low resistance state (LRS) steadily, and both the HRS and LRS are nonvolatile. LRS is commonly used as the ON state and HRS as the OFF state. Resistive switching is classified into two types: unipolar and bipolar. When LRS and HRS are seen at the same voltage polarity it is called unipolar switching and when they are seen at different voltage polarity then it is called bipolar switching. Binary digital data can be assigned to these resistive states. For example, LRS is assigned as logic "0" and HRS as logic "1". Figure 6.2 shows a current-voltage (I-V) plot of the same IDA device as in Figure 6.1, but after Ag NP chain formation. Frames A-C are the first three cycles of the device. The device shows low resistance and high resistance states on each I-V plot. The red arrows indicate the forward scan and black arrows indicate the reverse scan. In the Figure 6.2 A, the device initially shows low resistance ohmic behavior from 0 to $-4 \mathrm{~V}$ with some fluctuations in the current indicating that the Ag NP chain is not completely stable at those voltages. Near $-4 \mathrm{~V}$, the current drops dramatically, putting the chains into a HRS until about $-4.8 \mathrm{~V}$, where the current again increased dramatically to the LRS. The device remains in the LRS on the entire reverse scan. During the second scan (Figure 6.2 B), the LRS remains from $0 \mathrm{~V}$ to $-4.8 \mathrm{~V}$, where the device switches to HRS. It remains in the HRS state until about $-4.5 \mathrm{~V}$ 

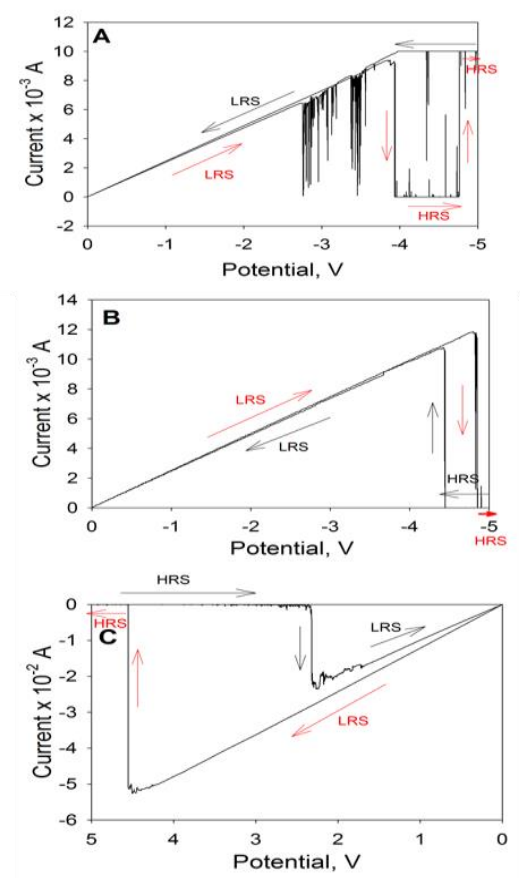

Figure 6.2. I-V characteristics of an IDA device with Ag deposition in presence of $\mathrm{CTAB}$ on both sides after applying a voltage of $5 \mathrm{~V}$ from 0 to $1000 \mathrm{~s}$ on a $\mathrm{Au} /$ dithiol/Au NPs device. The device was scanned from $0 \mathrm{~V}$ to $-5 \mathrm{~V},-5 \mathrm{~V}$ to $0 \mathrm{~V}$, 0 to $5 \mathrm{~V}$ and $5 \mathrm{~V}$ to $0 \mathrm{~V}$. (A) The device is at LRS initially and goes to HRS from $4 \mathrm{~V}$ to $-4.8 \mathrm{~V}$ on forward scan and later back to LRS from $-5 \mathrm{~V}$ to $0 \mathrm{~V}$ on reverse scan. (B) The device shows distinct LRS and HRS with LRS from 0 to $-4.8 \mathrm{~V}$ on forward scan and HRS from $-5 \mathrm{~V}$ to $-4.5 \mathrm{~V}$ and again LRS from $-4.5 \mathrm{~V}$ to $0 \mathrm{~V}$ on reverse scan. (C) The device showing LRS and HRS with LRS from 0 to $4.8 \mathrm{~V}$ on forward scan and HRS from $5 \mathrm{~V}$ to $2.2 \mathrm{~V}$ and again LRS from $2.2 \mathrm{~V}$ to $0 \mathrm{~V}$ on reverse scan. Black arrows indicate forward scan and red arrows indicate reverse scan. 
on the reverse scan, where the current increases again and it is in the LRS. The device seems to be improving stability from 0 to $-5 \mathrm{~V}$ during these two scans. In the third scan (Figure 6.2 C), we cycled from 0 to $+5 \mathrm{~V}$. The device was in the LRS from 0 to $4.5 \mathrm{~V}$, where it then switched off to the HRS. On the reverse scan it remained in the HRS until about $2.2 \mathrm{~V}$, where it switched back to the LSR for the remainder of the scan. This type of behavior in Figure $6.2 \mathrm{C}$ is excellent for resistive switching devices because one can choose $3.5 \mathrm{~V}$ as a "read" voltage. If you approach $3.5 \mathrm{~V}$ going from $0 \mathrm{~V}$ to $3.5 \mathrm{~V}$ on the forward scan, then the device is in the LRS at the "read" voltage of $3.5 \mathrm{~V}$. If you approach $3.5 \mathrm{~V}$ going from 5.0 $\mathrm{V}$ on the reverse scan, then the device is in the HRS at the "read" voltage. The sequential I-V scans show that the device can exhibit stable unipolar resistance switching behavior under positive voltage in this case.

\section{Investigation of memory characteristics of Ag nanoparticle chains formed across the gap of IDA electrodes.}

Figures 6.3 shows the switching properties of the device after performing the I-V scans in Figure 6.2 by monitoring the current as a function of time while rapidly switching the voltage between LRS and HRS conditions. The voltages chosen were based on the I-V scans of the device. From the I-V scans, the device showed $\mathrm{ON}$ state at $0.5 \mathrm{~V}$ and $\mathrm{OFF}$ state at $5 \mathrm{~V}$. Figure 6.3 shows the current as a function of time as we switched between these two voltages for 20 cycles at switching speeds of $0.025 \mathrm{~s}$ and $0.0025 \mathrm{~s}$ in Frames A and B, respectively. Both speeds shows distinct LRS (“ON") and HRS (“OFF") conditions. 

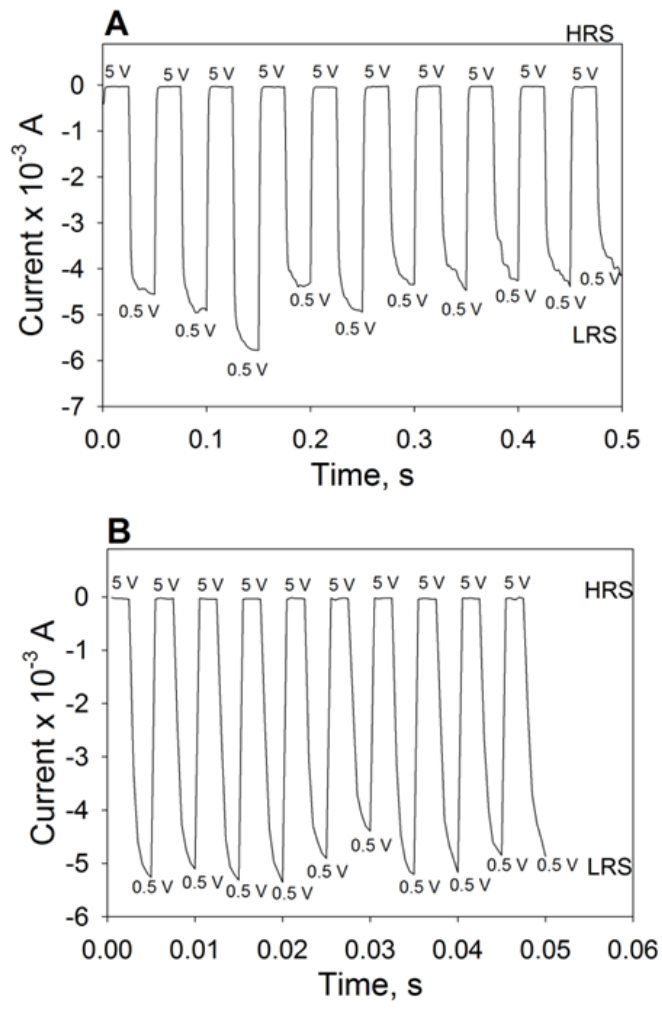

Figure 6.3. Au/Ag NP chain/Au device tested for 20 cycles with switching times (A) $0.025 \mathrm{~s}$ and (B) $0.0025 \mathrm{~s}$ showing ON/OFF ratio $\sim 10^{2}$ where HRS $=3-4 \times 10^{-5}$ A and LRS $=4-6 \times 10^{-3} \mathrm{~A}$. 
Figure 6.4 shows the magnified current level of the $\mathrm{ON}$ and OFF states at $0.5 \mathrm{~V}$ and $5 \mathrm{~V}$, Frames $\mathrm{A}$ and $\mathrm{B}$ where we can estimate the $\mathrm{ON} / \mathrm{OFF}$ current ratio to be about 100 .

The device endurance is an important parameter for evaluating memory performance. Figure 6.5 shows endurance studies for the same device. The device was switched from $0.5 \mathrm{~V}$ to $5 \mathrm{~V}$. Figure $6.5 \mathrm{~A}$ shows the first 20 cycles and Figure 6.5 B shows the last 20 cycles out of 1000 cycles total. Throughout the completion of 1000 cycles, the device showed consistent switching behavior with an ON/OFF ratio $>10$. Figure 6.6 shows the current level of the device in the OFF state and ON state showing some decrease in the ON/OFF ratio. Figure 6.7 shows the SEM images of 3 different IDA devices. Figure 6.7 A shows the bare SEM image of Au IDA, Figure 6.7 B shows the SEM image after Ag deposition in presence of CTAB on both sides of the device and Figure 6.7 C shows the SEM image of an IDA after forming $\mathrm{Ag}$ NP chains and performing the switching operations as shown in Figure 6.3 and Figure 6.5. We observed that the chains are responsible for the switching behavior.

\subsubsection{Resistive switching of Ag nanoparticle chains formed across the $5 \mu \mathrm{m}$ gap of TED}

Ag electrodeposition and chain formation We also carried out switching experiments with two electrode devices (TED) having Ag just on one side of the device. After Ag deposition on one side of the device, we took the device and 

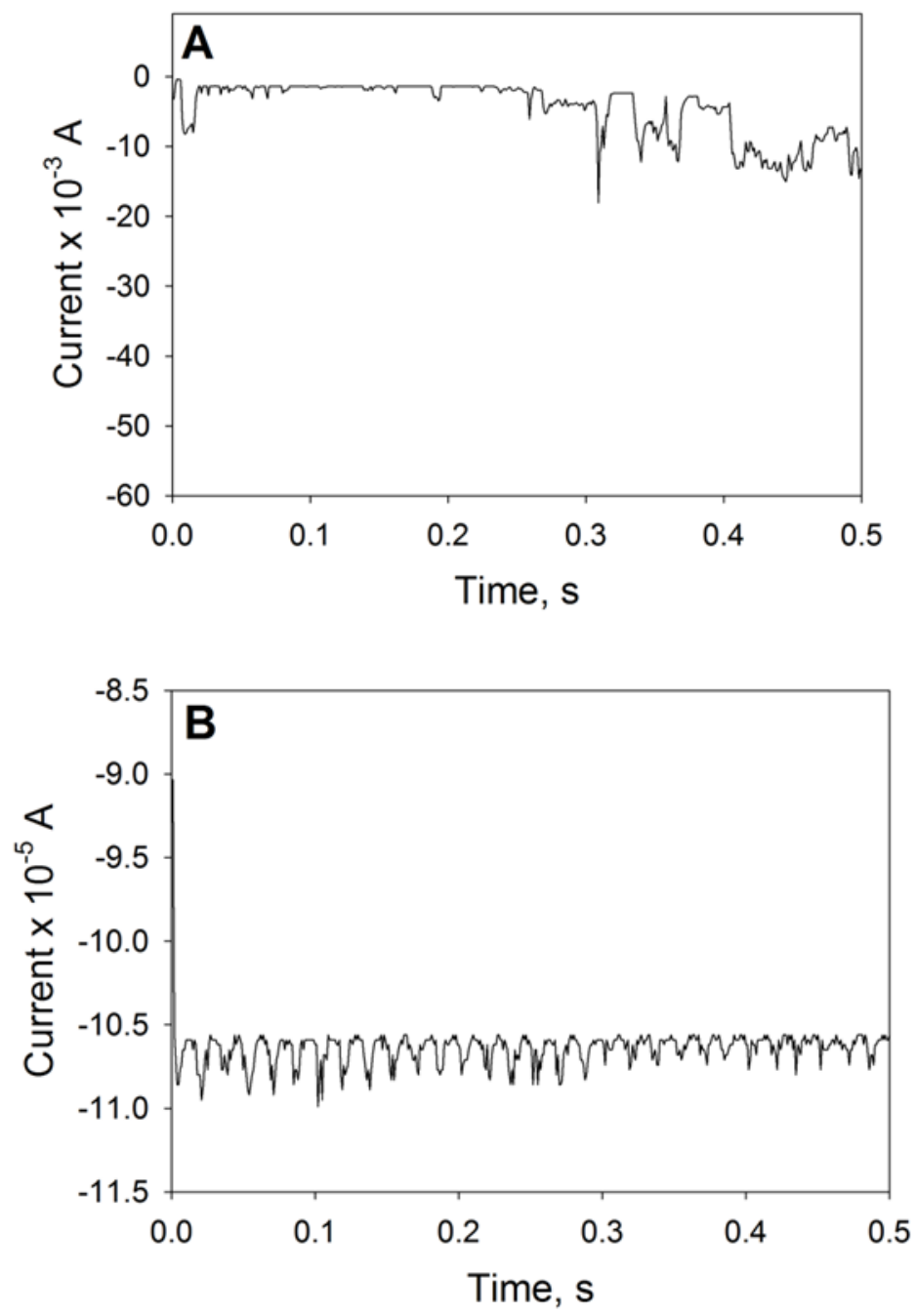

Figure 6.4. Current levels of the device after performing switching speed studies (A) ON state and (B) OFF state. 

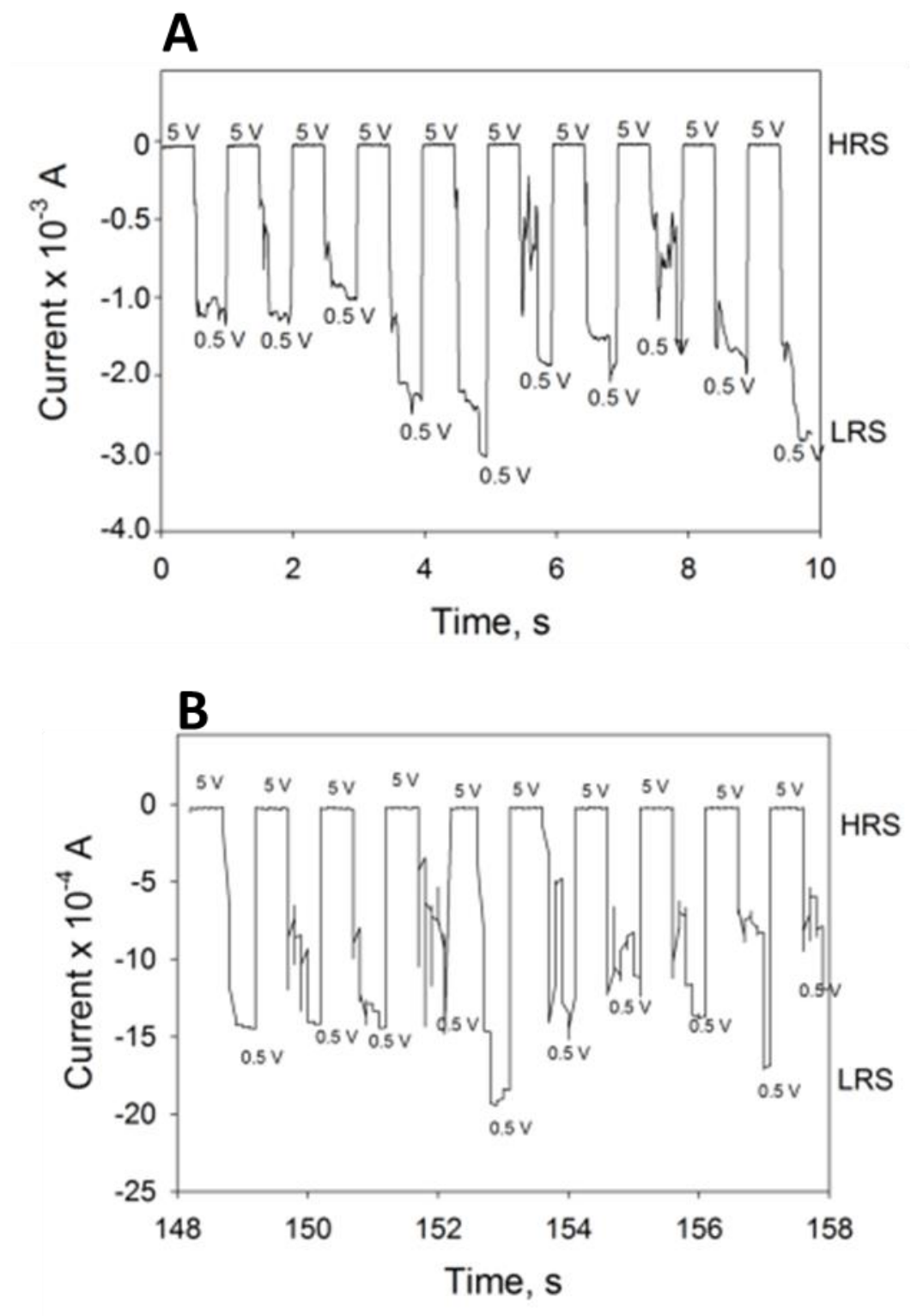

Figure 6.5. Endurance studies for the Au/Ag NP chain/Au device showing the (A) first 20 cycles and (B) last 20 cycles of 1000 cycles total where HRS $=3-5 \times 10^{-4}$ A and LRS $=1-6 \times 10^{-3} \mathrm{~A}$. 

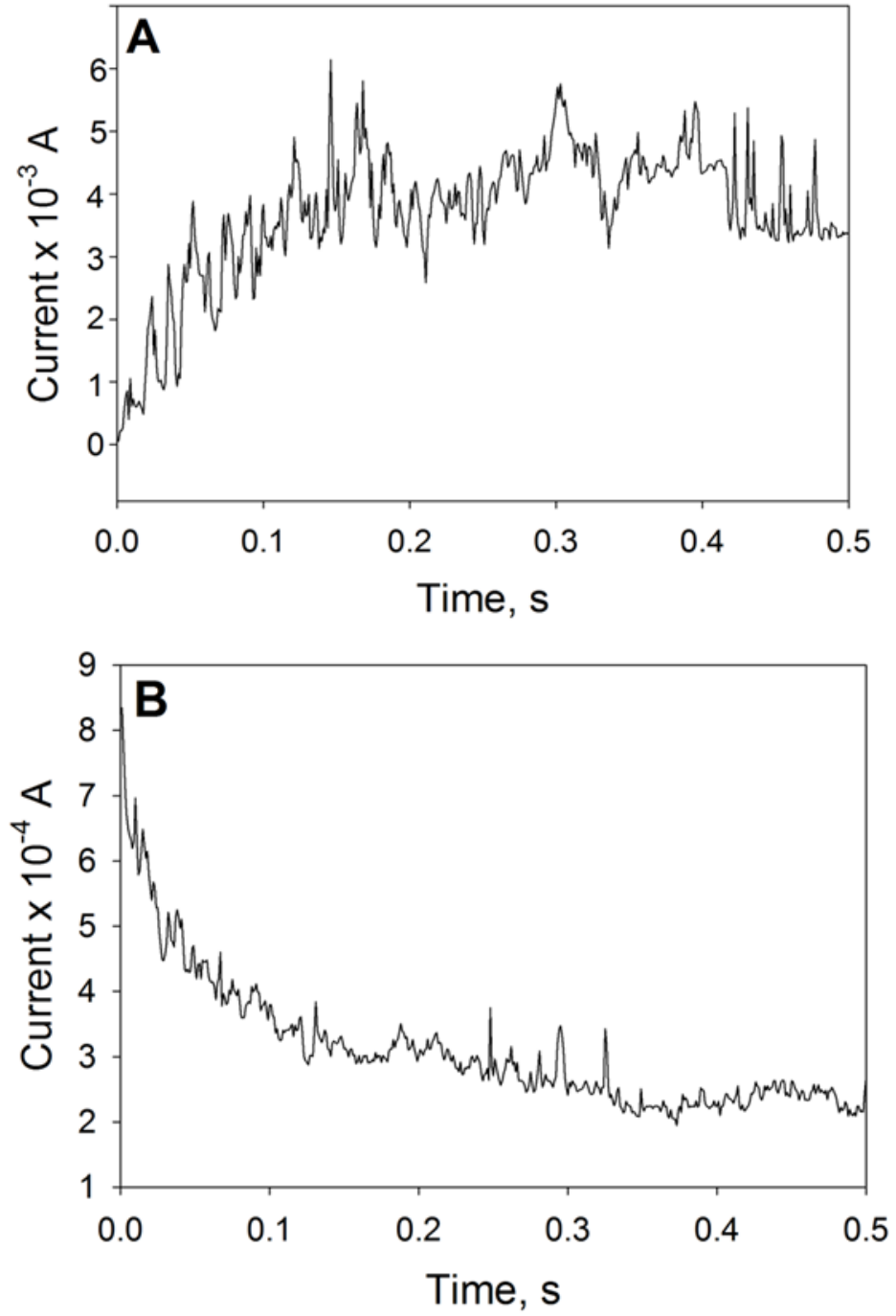

Figure 6.6. Current levels of the device after performing enudurance studies (A) ON state and (B) OFF state. 

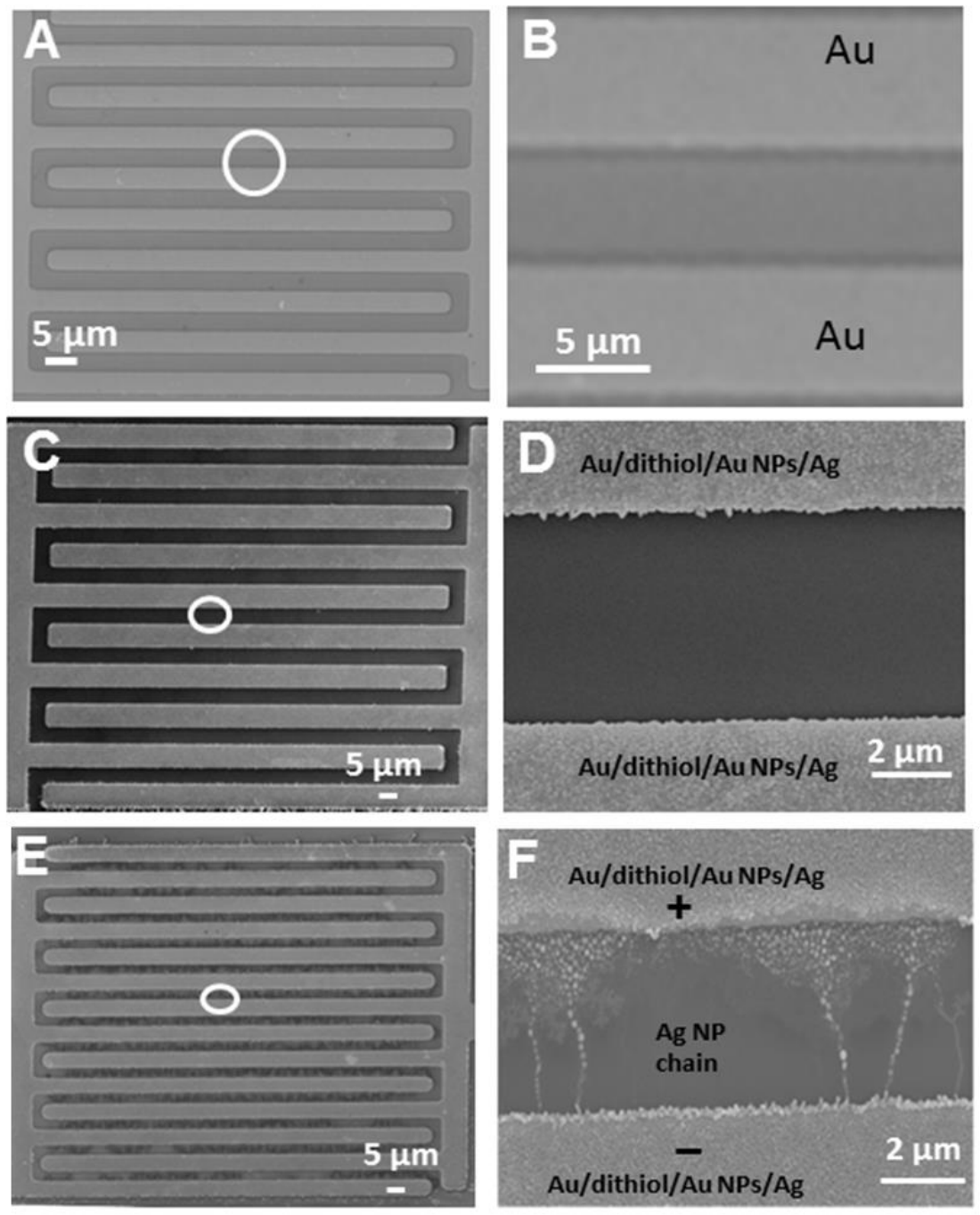

Figure 6.7. SEM images of the device before and after performing chain formationand switching operations. (A) SEM image of the bare IDA device, (C) SEM image of $\mathrm{Au} / \mathrm{dithiol} / \mathrm{Au}$ NPs after electrodeposition of $\mathrm{Ag}$ on both sides in the presence of CTAB and (E) SEM image of Au/Ag NP/Au IDA device after chain formation and the switching operations. (B, D, F) show zoomed in regions of the circles shown in (A, C, E). 
applied voltages from 0 to $10 \mathrm{~V}$ to observe the chain formation as shown in Figure 6.8 .

\section{Resistive switching of Ag nanoparticle chain formed at the gap of TED. As} explained before for IDA devices, we performed I-V scans on the TED device (Figure 6.9). We applied negative voltages initially from 0 to $-5 \mathrm{~V}$ and $-5 \mathrm{~V}$ to 0 $\mathrm{V}$. The device showed LRS initially from $0 \mathrm{~V}$ to $-2.5 \mathrm{~V}$ and HRS from $-2.5 \mathrm{~V}$ to $5 \mathrm{~V}$ during the forward scan. On the reverse scan, it switched to LRS near $-3.5 \mathrm{~V}$. There were several current fluctuations from $-2.5 \mathrm{~V}$ to $-3.5 \mathrm{~V}$ on both the forward and reverse scan. On the second scan, there were fewer fluctuations and the switch from LRS to HRS and vice-versa was near $-3 \mathrm{~V}$ in both scan directions. This is not as desirable since a separate read voltage is difficult to determine.

We carried out switching cycles at $-0.5 \mathrm{~V}$ and $-5 \mathrm{~V}$. We observed that the device did not show consistent ON and OFF states. At the start, it was in HRS at $5 \mathrm{~V}$, but then switched to ohmic behavior. At the end, it again showed switching from HRS at $-5 \mathrm{~V}$ to LRS at -0.5 Vas seen in Figure 6.10.

We then obtained an I-V scan at positive voltage. As shown in Figure 6.11, this scan led to a device with good resistive switching and hysteresis between the forward and reverse scans that are needed for a read voltage. The device showed unipolar stable switching behavior with HRS and LRS retained over a larger voltage range similar to the IDA device . 


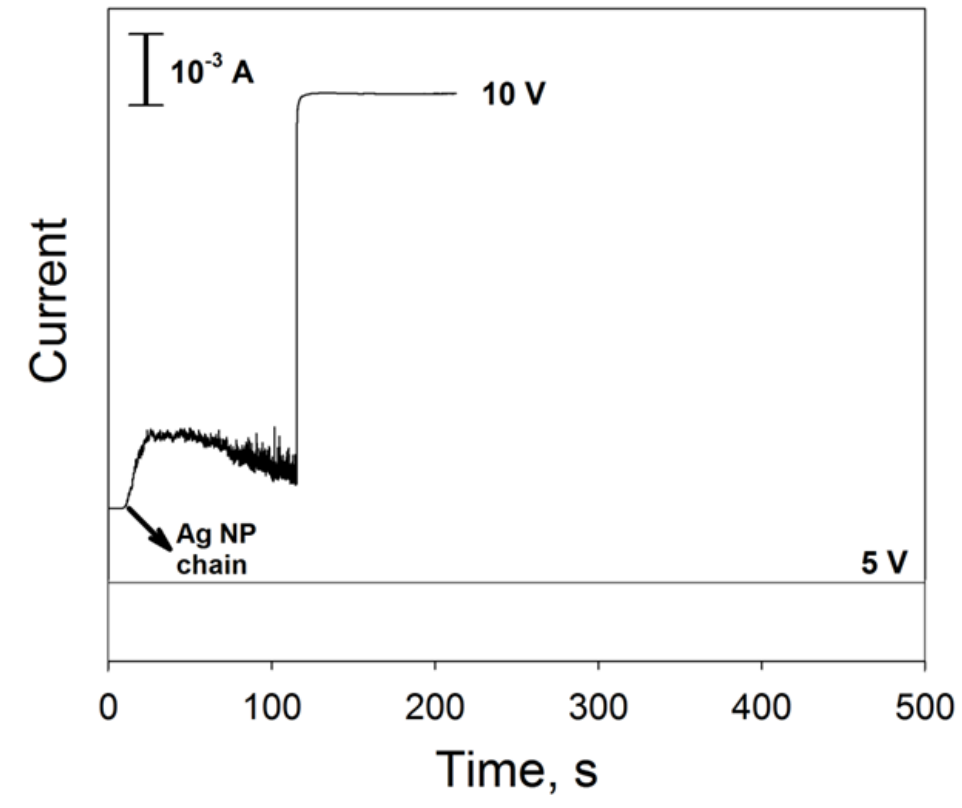

Figure 6.8. Current-time plot obtained at the indicated the voltage on an IDA device with $\mathrm{Ag}$ deposited in the presence of $\mathrm{CTAB}$ on one side of $\mathrm{Au} / \mathrm{dithiol} / \mathrm{Au}$ NPs devices. 

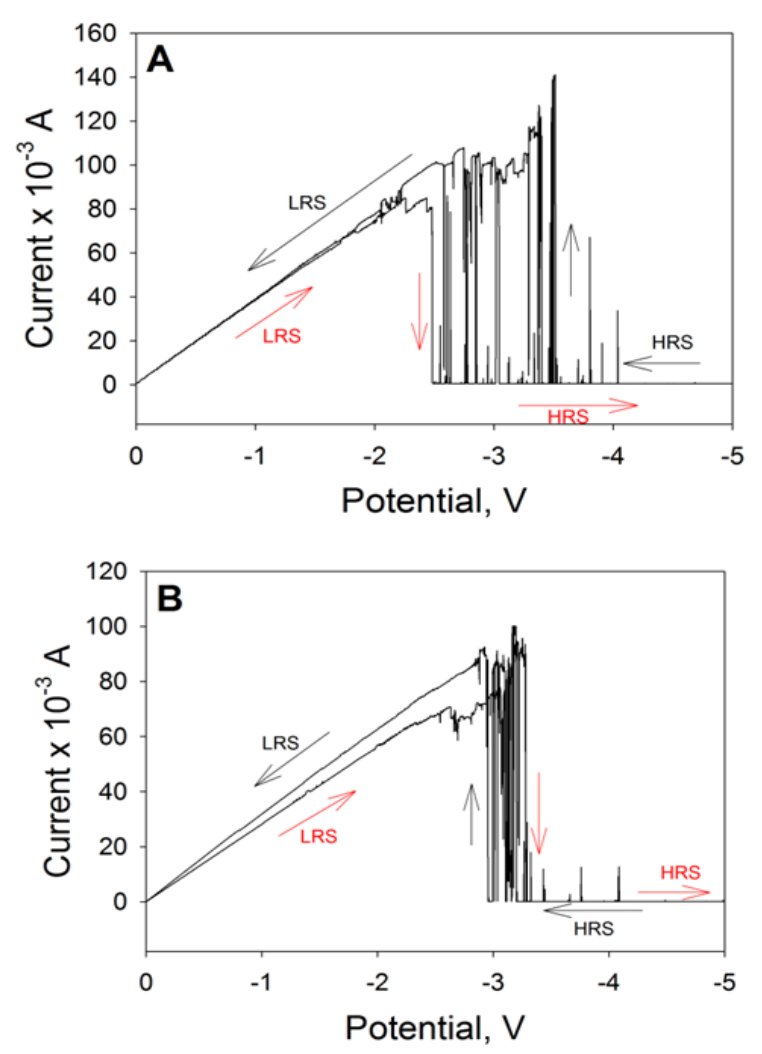

Figure 6.9. I-V scans after the formation of Ag NP chain where Ag side is positive and Au side is negative. (A) Device is at LRS from 0 to $-2.5 \mathrm{~V}$ and HRS from -2.5 $\mathrm{V}$ to $-5 \mathrm{~V}$ in the forward scan. Device shows HRS from $-5 \mathrm{~V}$ to $-3.5 \mathrm{~V}$ and remains LRS throughout the completion of the cycle on reverse scan. (B) Device at LRS state from 0 to $-3.2 \mathrm{~V}$ and HRS from $-3.2 \mathrm{~V}$ to $-5 \mathrm{~V}$ in the forward scan. In the reverse scan, device is at HRS from $-5 \mathrm{~V}$ to $-3 \mathrm{~V}$ and remain in LRS throughout the cycle. In both cases, the device shows unipolar behavior where HRS and LRS can be seen at the same polarity. The lack of hysteresis in the forward and reverse scan makes it difficult to identify a good read voltage. 


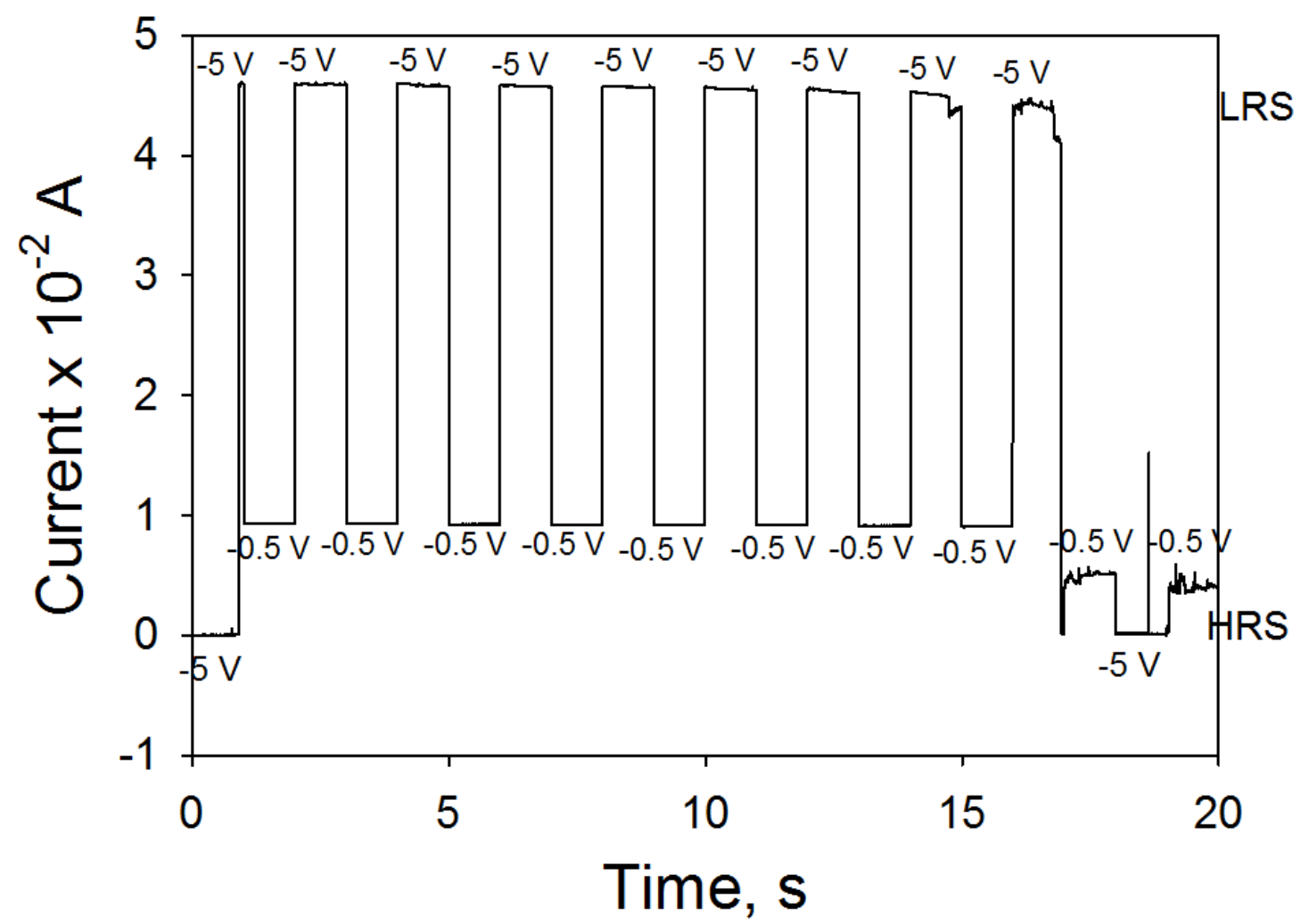

Figure 6.10. Current-time plot of Au/Ag NP chain/Au TED performed after I-V scans shown in Figure 6.9. The device was switched from $-0.5 \mathrm{~V}$ to $-5 \mathrm{~V}$ for 20 cycles with a pulse width of $1 \mathrm{~s}$. The device is ohmic for most switches and not showing resistive switching behavior. It is in LRS at both $-0.5 \mathrm{~V}$ and $-5 \mathrm{~V}$. 


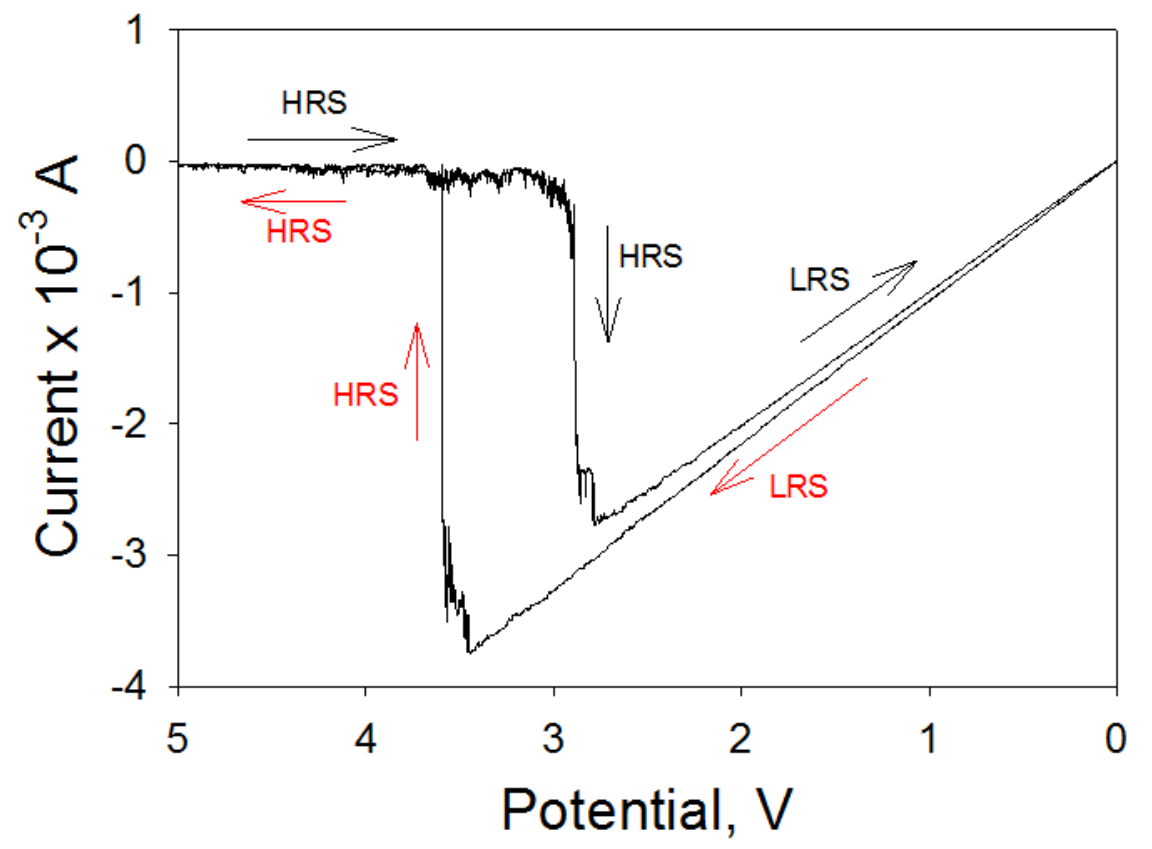

Figure 6.11. I-V plot after performing I-t plot as shown in Figure 6.10. Device showed unipolar switching with distinct ON and OFF state and nice hysteresis necessary for identifying a read voltage. Ag side was kept positive and Au side was kept negative during switching. 
Figure 6.12 shows the switching properties of the TED device after the positive I$\mathrm{V}$ scans. We carried out these experiments at voltages of $0.5 \mathrm{~V}$ and $5 \mathrm{~V}$ with speeds of $0.025 \mathrm{~s}$ (Frame A) and $0.25 \mathrm{~s}$ (Frame B), which the device showed good switching behavior for most of the voltage applications. Figure $6.12 \mathrm{C}$ shows the current level of the $\mathrm{ON}$ and $\mathrm{OFF}$ states at $0.5 \mathrm{~V}$ and $5 \mathrm{~V}$ from where we can calculate $\mathrm{ON} / \mathrm{OFF}$ ratio to be 100 . Next, we evaluated the endurance of the same device. We observed that the LRS showed spikey behavior but retained the ON/OFF ratio as shown in Figure 6.13. The device showed stable behavior throughout the 1000 cycles but we observed spikey behavior in the LRS. The device retained ON/OFF ratio of 100 throughout the cycles as shown in the Figure 6.14 .

Figure 6.15 shows the SEM images of 3 different TEDs. The bare TED as shown in Figure 6.15 A, shows a clean image of the device. Figure $6.15 \mathrm{~B}$ shows the TED after Ag deposition, showing no connection across the gap. Figure $6.15 \mathrm{C}$ shows an SEM image after forming chains and performing all the switching operations on the device. The chains at the electrode gap are responsible for switching the device from HRS to LRS and LRS to HRS.

\subsubsection{Mechanism of Resistive switching of Ag nanoparticle chains}

It is very important to determine the mechanism of resistive switching. We know that Ag NP chain formation occurs in the presence of humid air and surfactant $\mathrm{CTAB}$ and is therefore electrochemical in nature. ${ }^{40}$ 

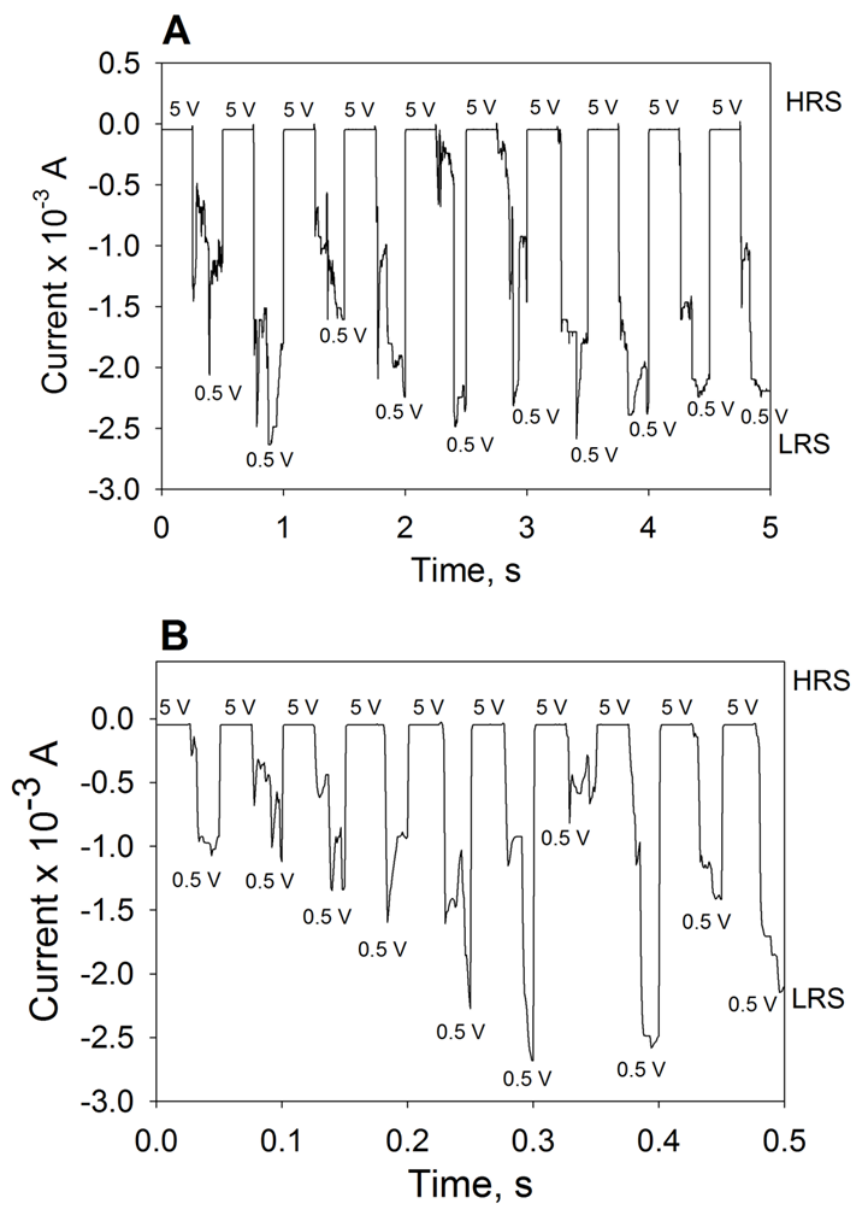

Figure 6.12. Au/Ag NP chain/Au device tested for 20 cycles with switching times of (A) $0.025 \mathrm{~s}$ and, (B) $0.25 \mathrm{~s}$. The ON/OFF ratio $\sim 10^{2}$, HRS $=2-4 \times 10^{-5} \mathrm{~A}$, and LRS $=1-3 \times 10^{-3} \mathrm{~A}$. 

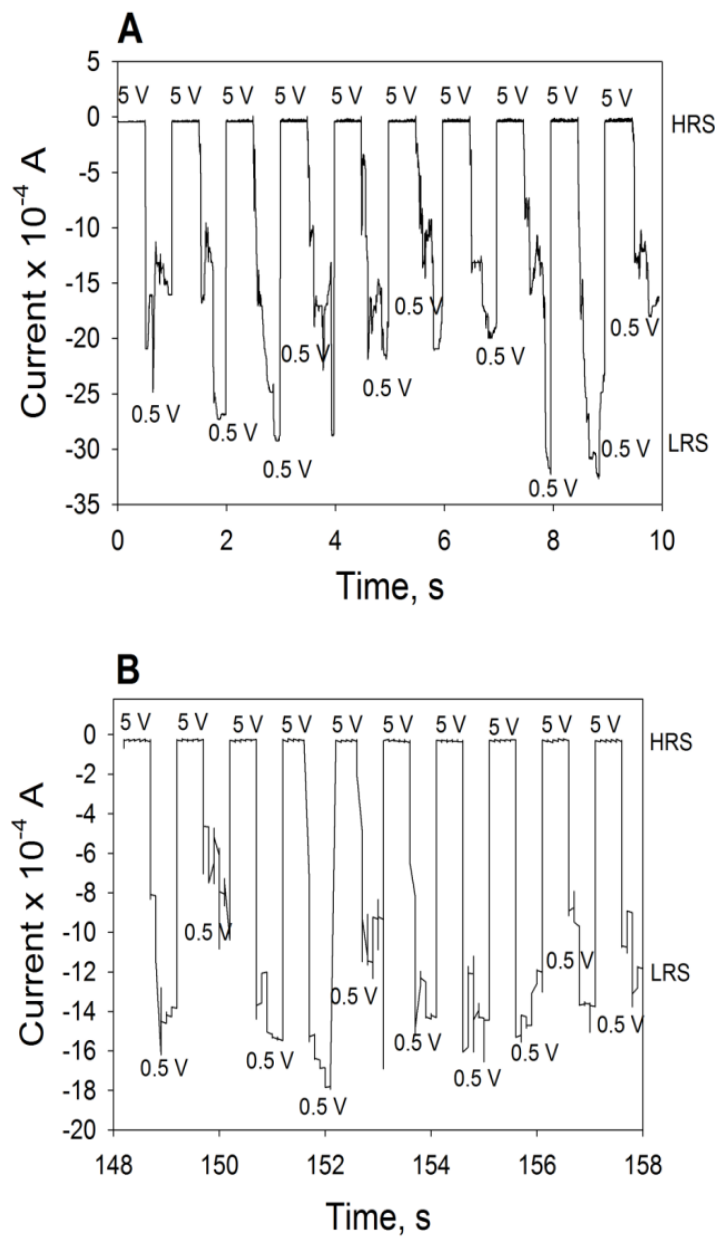

Figure 6.13. Endurance studies for the Au/Ag NP chain/Au device with Ag on one side with switching time of $0.5 \mathrm{~s}$ (A) First 20 cycles of 1000 cycles and (B) last 20 cycles of 1000 cycles. HRS $=3-5 \times 10^{-5} \mathrm{~A}$ and LRS $=0.8-3 \times 10^{-3} \mathrm{~A}$. 

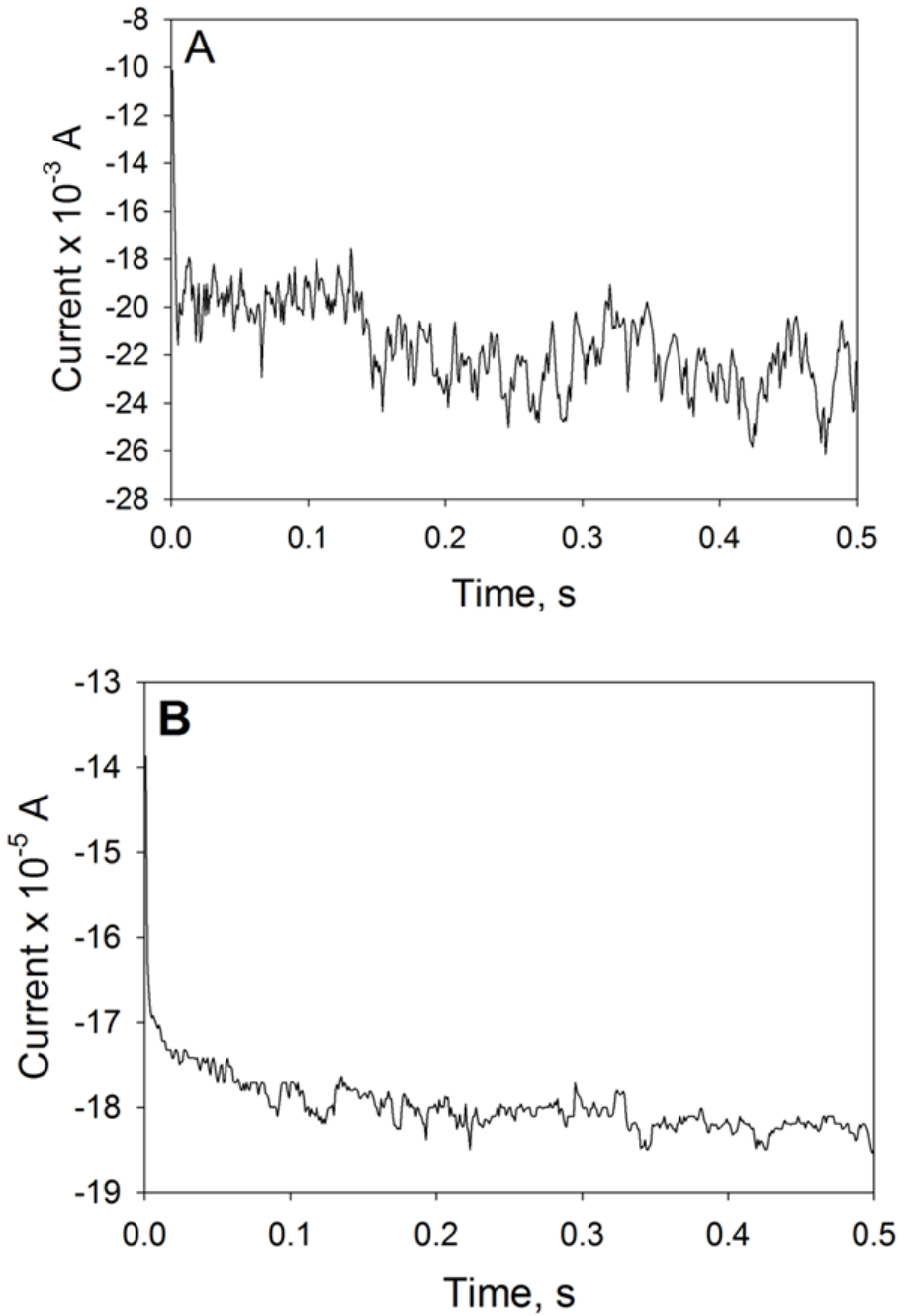

Figure 6.14. Current levels of the device after performing switching operations (A) ON state and (B) OFF state. ON to OFF ratio is $10^{2}$. 

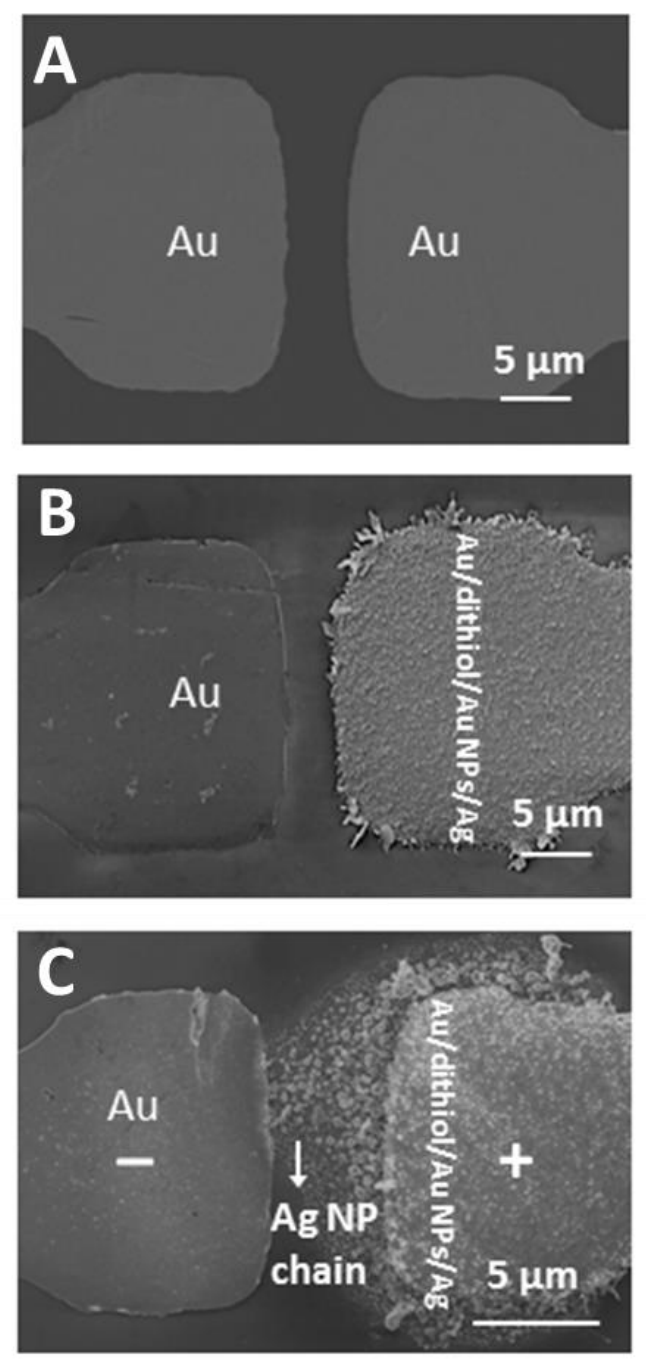

Figure 6.15. SEM images of the TED device before and after chain formation and switching operations (A) Bare TED, (B) Au/dithiol/Au NPs TED with Ag electrodeposited on one side of the device having Au NPs and dithiol, and (C) $\mathrm{Au} / \mathrm{Ag}$ NP chain/Au TED after performing switching operations. 
To determine the mechanism of the resistive switching, we carried out experiments in dry nitrogen environment and without CTAB. We deposited Ag on $\mathrm{Au} / \mathrm{dithiol} / \mathrm{Au}$ NPs on one side of the TED. After the Ag NP chain formation shown in Figure 6.16, we performed I-V studies as shown in Figure 6.17 and then carried out switching cycles. Figure 6.18 A shows the switching cycles in dry $\mathrm{N}_{2}$. We switched the device from $0.5 \mathrm{~V}$ to $5 \mathrm{~V}$ at $0.5 \mathrm{~s}$. From these results, we can say that the resistive switching mechanism does not require humidity. We carried out another control experiment to determine if the surfactant is necessary to carry out switching. Figure $6.18 \mathrm{~B}$ shows the switching cycle after the $\mathrm{Au} / \mathrm{Ag} \mathrm{NP}$ chain/Au device was soaked in water for two hours. Our result shows that surfactant is also not required for switching. Both humidity and surfactant are not necessary to carry out switching cycles.

From these experiments, we conclude that the resistive switching is not an electrochemical process. We instead believe that atomic Ag migrates at the Ag NP junctions of the chain, leading to high and low resistance states. Scheme 6.1 illustrates the possible mechanism, where atomic Ag bridges form in the chain during the LRS and there are breaks between the Ag NPs during the HRS. 


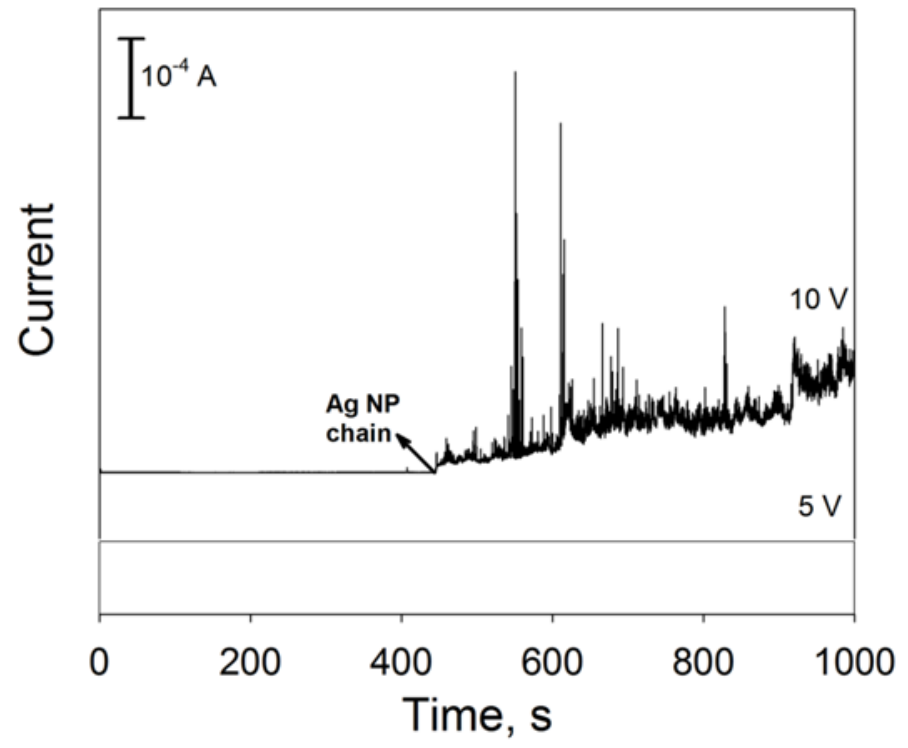

Figure 6.16. Au/dithiol/Au NP IDA device with Ag deposited on one side of the device showing the formation of chains at $10 \mathrm{~V}$. 

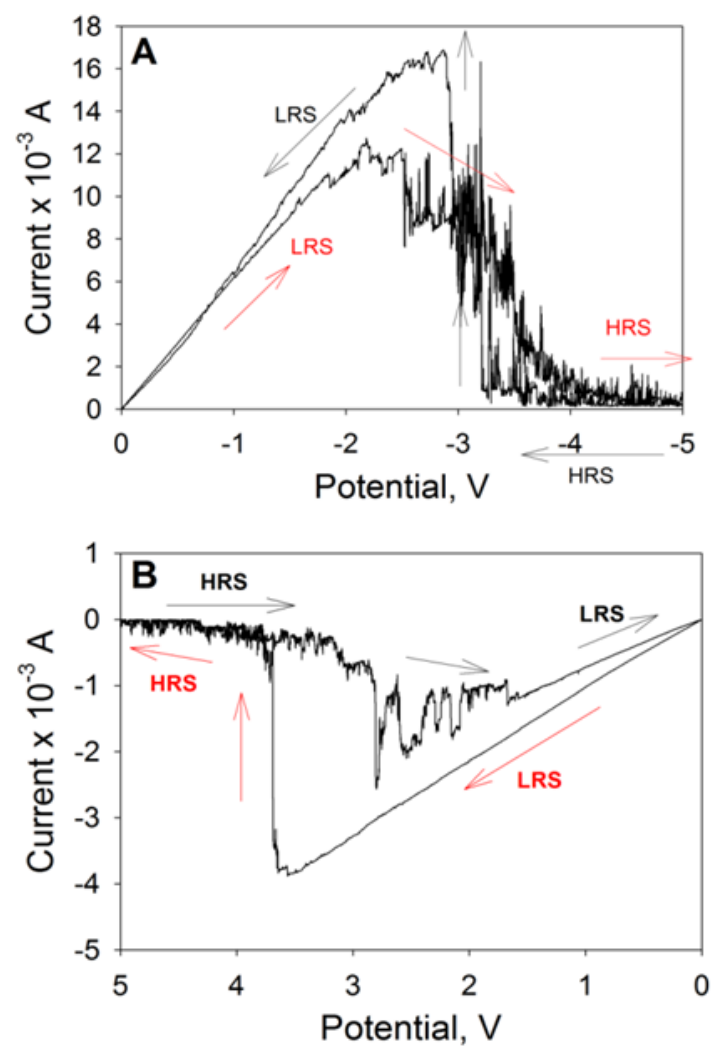

Figure 6.17. I-V plots of the device after Ag NP chain formation as shown in Figure 6.16. (A) Device shows LRS from 0 to $-4 \mathrm{~V}$ with current showing spikes and HRS from $-4 \mathrm{~V}$ to $-5 \mathrm{~V}$ in forward scan. Current decays with spikes but does not turn OFF completely. During reverse scan, device shows HRS from $-5 \mathrm{~V}$ to 3.5 $\mathrm{V}$ and remains LRS thereafter throughout the completion of the cycle. (B) Positive scan showing good switching behavior with hysteresis. 

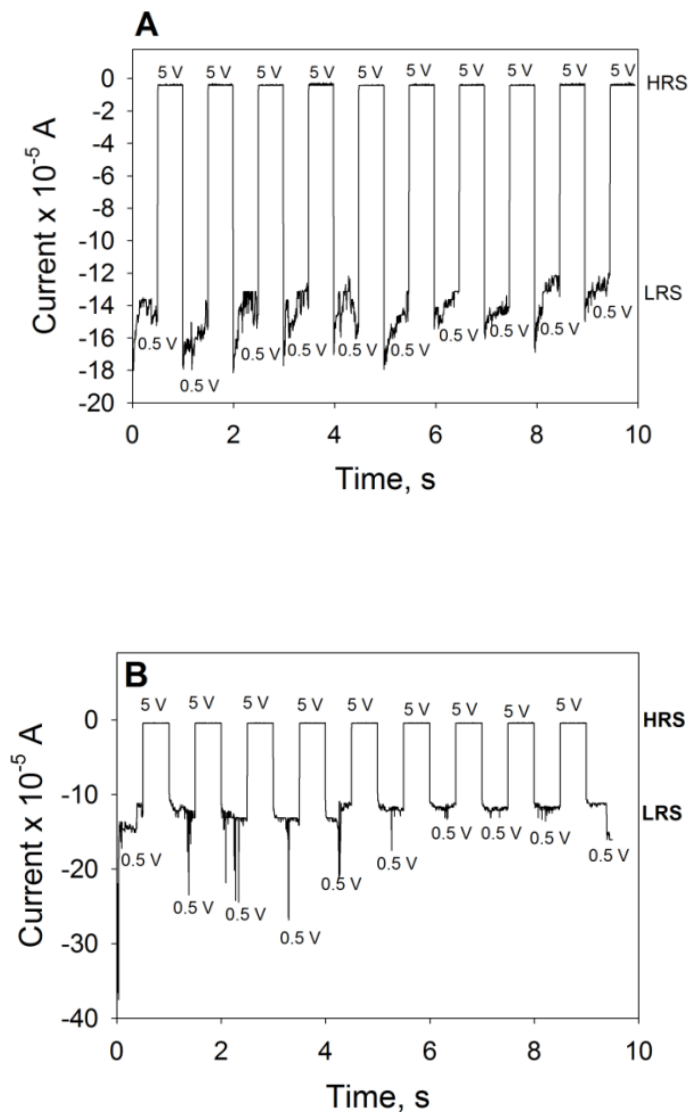

Figure 6.18. Au/dithiol/Au NPs IDA device with Ag on one side of the device. (A) Switching in dry $\mathrm{N}_{2}$ from $0.5 \mathrm{~V}$ to $5 \mathrm{~V}$ for 20 cycles at $0.5 \mathrm{~s}$ pulse width, (B) Switching in the absence of CTAB in air by soaking the device in water for two hours. Device switched from $0.5 \mathrm{~V}$ to $5 \mathrm{~V}$ for 20 cycles at $0.5 \mathrm{~s}$ pulse width. 


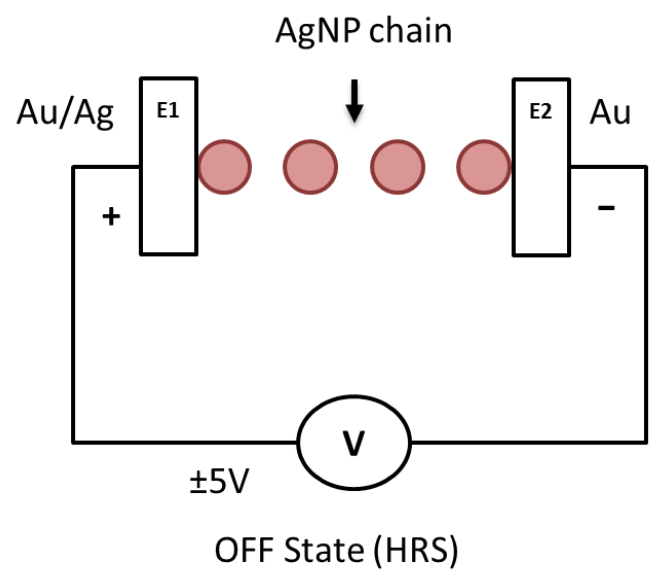

Ag NPs

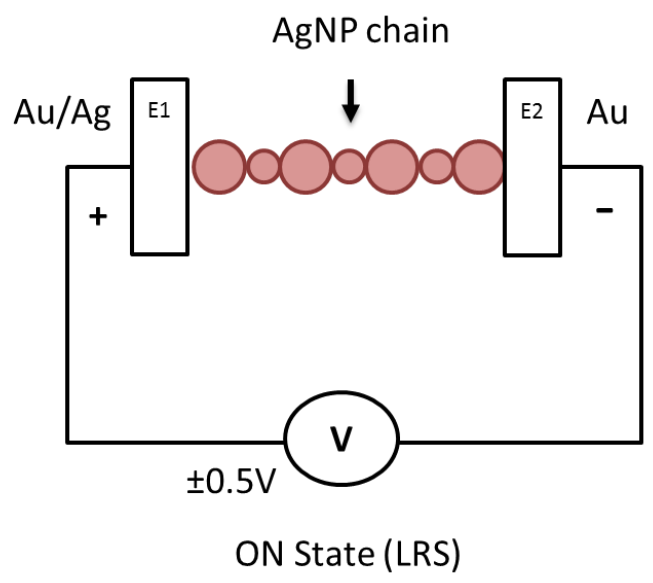

Ag atoms

Scheme 6.1. Resistive switching mechanism showing the ON and OFF states for the $\mathrm{Au} / \mathrm{Ag}$ NP chain/Au devices. Mechanism involves atomic dissolution and migration to form connetions and breaks at specific voltages. 


\subsection{Conclusions}

In conclusion, reliable controllable and stable resistive switching behavior was investigated for the IDA and TED devices. The device could be made to switch from LRS to HRS by going from $\pm 0.5 \mathrm{~V}$ to $\pm 5 \mathrm{~V}$. The devices with the positive voltage on the $\mathrm{Au}$ (or Ag) side showed the best resistive switching behavior, including large hysteresis in the I-V curve. The switching cycles for the device is at least 1000 cycles with switching speed $\sim 0.0025 \mathrm{~s}$. With Ag on one side or both sides, switching ratio, speed and endurance was found to be similar. We determined the resistive switching mechanism by modifying the important parameters responsible for the formation of chains. We found that the resistive switching occurs by atomic migration as opposed to an electrochemical process. The device operates through the formation and dissolution of an atomic bridge between the Ag NPs in the chain, and behaves as an atomic switch. 
CHAPTER VII

OPTICAL CHARACTERIZATION OF 1D AG NANOCHAINS USING

SURFACE ENHANCED RAMAN SPECTROSCOPY AND RAYLEIGH DARK-

\section{FIELD SCATTERING SPECTROSCOPY}

\subsection{Introduction}

SERS of $1 D$ chains. Metal nanostructures of Au and Ag can be readily used as SERS substrates. SERS mechanism is believed to be due to a combination of an electromagnetic field enhancement of metal nanostructures and a chemical effect of molecule coated on nanostructures. 1D chains show good SERS enhancement. Guo et al studied SERS enhancement of Au NP assemblies coated with glutamic acid. ${ }^{241}$ SERS enhancement was observed due to the local electromagnetic field enhancement of assembled Au nanostructures. These Au NP assemblies have a great number of particle junctions that act as "hot sites" and causes electromagnetic field enhancement. Further, the plasmon coupling of Au NPs at these hot spots produces strong SERS signal. Ohara et al studies the SERS of linear chains of Ag on SWCNTs coated with sodium dodecyl sulphate (SDS). They also observed strong SERS signals for Ag NP chains. The explanation provided is similar to that provided by Guo et al. Large Raman enhancement was observed due to strong field coupling between the NPs. SDS coated on NPs shortens the distance between the Ag NPs and because of this close proximity a strong field coupling is observed. However, according to the previous studies, a 
strong increase in electromagnetic field enhancement occurs when the distance between the NPs is shorten to few nanometers and when the incident light is polarized parallel to the NP chain axis. ${ }^{242}$

Due to strong SERS signal of 1D chains, it is useful to study the combined SERS and conductivity to understand the resistive switching mechanism in detail. Wong et al studied the conductivity behavior of metal/oxide/metal system coupled with Raman spectroscopy. Oxygen movement (CF in case of metal/oxide/metal systems) was monitored by Raman spectroscopy under the applied potential. They observed significant changes in the Raman intensity at ON and OFF states of the device. They concluded that SERS could be a good technique to understand the formation of the CFs, such as how the filament begins to grow during the SET process and how the conducting channel is disconnected during the RESET process.

LSPR of 1D chain. A Recent review describes the optical properties of plasmonic nanoparticles studies by several single particle spectroscopy techniques. ${ }^{243}$ Nanostructures made up of $\mathrm{Cu}, \mathrm{Ag}$, and $\mathrm{Au}$ interacts strongly with light. ${ }^{244-247}$ The spectra characteristics of the localized surface plasmon resonance are determined by size, shape and material of the nanoparticle. Several groups studied the plasmonic properties of 1D assemblies for example, Mulvaney et al. studied the light scattering properties of linear chains of Au nanoparticles. They observed a red shift of the surface plasmon resonance with increasing chain length. ${ }^{157}$ Recently, Fery et al investigated the plasmonic properties of 
nanoparticle chains formed by template-assisted self-assembly. ${ }^{158}$ They investigated the optical properties of assemblies of single particle chains, dimer chains and tetramer chains. They found a remarkable red shift of the main resonance from around $500 \mathrm{~nm}$ for isolated particles up to $1500 \mathrm{~nm}$ for nanoparticle chain assemblies of varied chain length giving rise to far-field UVvis/NIR spectra. These shifts are due to dipolar coupling, which increases with an increasing number of NPs in the chain, leading to the large red shifts with longer chains.

Here we demonstrate the analytical applications of 1D Ag NP chains to understand the usefulness of our fabrication method for potential SERS and plasmonics applications. We present an approach that allowed recording Raman measurements coupled with electrical conductivity measurements of nanoparticles in a nanoparticle chain across the gap coated with 4-aminothiophenol (4-ATP). $\mathrm{Au} / \mathrm{Ag}$ NP chain/Au TED was fabricated and later soaked in ATP where ATP gets adsorbed over the whole device. While performing surface enhanced Raman spectroscopy (SERS) of our device, we measure the signals for 4,4' dimercaptoazobenzene $\left(4,4^{\prime}\right.$-DMAB) which is the oxidized form of ATP. Recently, however new researchers found that the b2-type bands in 4-ATP are not due to 4-ATP after all, but due to the Ag modes of 4,4'-dimercaptoazobenzene (4,4' -DMAB) produced from 4-ATP via a catalytic coupling reaction on metal substrates in the presence of the laser. ${ }^{248-250}$ SERS data on 4,4' -DMAB is limited. Later we carried out SERS on the 4,4' -DMAB and conductance measurements by 
switching the device from the HRS to LRS and vice-versa on applying potential. We observed changes in the intensity and arising of new peak in the SERS spectrum of 4,4' -DMAB when the Ag NP chain switches from a high resistance state (HRS) to a low resistance state (LRS). It could be due to a change in enhancement properties of $\mathrm{Ag}$ when in conducting state.

We studied the light scattering properties of Ag nanoparticle chains across the gap prepared by the simple procedure described in Chapter V. We observed localized surface plasmon modes in the NIR region similar to previous

studies. ${ }^{5,157,158}$ We present experimental data on the light scattering properties of linear chains of Ag nanoparticles. This study will further help in correlating the wavelength shift with the conductance switching. Our goal was to study the scattering porperties of the chains and determine the effect of the chain conductance on LSPR properties.

\subsection{Experimental Section}

Electrode wiring and cleaning procedure. Refer to Chapter II, section 2.3.3.

Au TED modification. Refer to Chapter II, section 2.3.4.

Electrodeposition of Ag on Au/TED. Refer to Chapter II, section 2.4.2.

Ag nanoparticle chain formation. Refer to Chapter II, section 2.4.4.

Resistive switching of Au/TED. Refer to Chapter II, section 2.4.5. 
Characterization of Au/Ag NP chain/Au coated with 4-ATP. Refer to Chapter II, section 2.2.3 and SEM as described in Chapter II, section 2.2.2

Simultaneous conductivity and Raman measurement experiments are described in Chapter II, section 2.2.4.

The procedure to perform LSPR of chains is described in Chapter II, section 2.2.5.

Fabrication of Electrode/Ag NP chain/Electrode junctions coated with 4ATP. After the formation of Ag nanoparticle chains across the gap of TED, we soaked the device for $20 \mathrm{~min}$ in $5 \mathrm{mM}$ ethanolic ATP solution.

\subsection{Results and Discussion}

As described in our previous method in Chapter V, we formed 1D Ag nanochains between two electrodes in the presence of CTAB and electric fields. Here we used Au/TED devices fabricated on glass for LSPR studies and on $\mathrm{Si} / \mathrm{SiO}_{2}$ for SERS studies. After the chains were formed across the gap, we obtained optical measurements. In this Chapter, we describe two experiments. One is the simultaneous conductance and Raman measurement of 1D Ag nanochains having the Raman active 4-ATP molecule adsorbed onto it and the other is the LSPR properties of 1D Ag nanochains. Figure 7.1 shows a currenttime plot of the IDA device for the Au/dithiol/Au NPs that had Ag electrodeposited on one side in the presence of CTAB. We first applied $5 \mathrm{~V}$ initially but did not observe any rise in current up to $1000 \mathrm{~s}$. Then we applied $10 \mathrm{~V}$ 


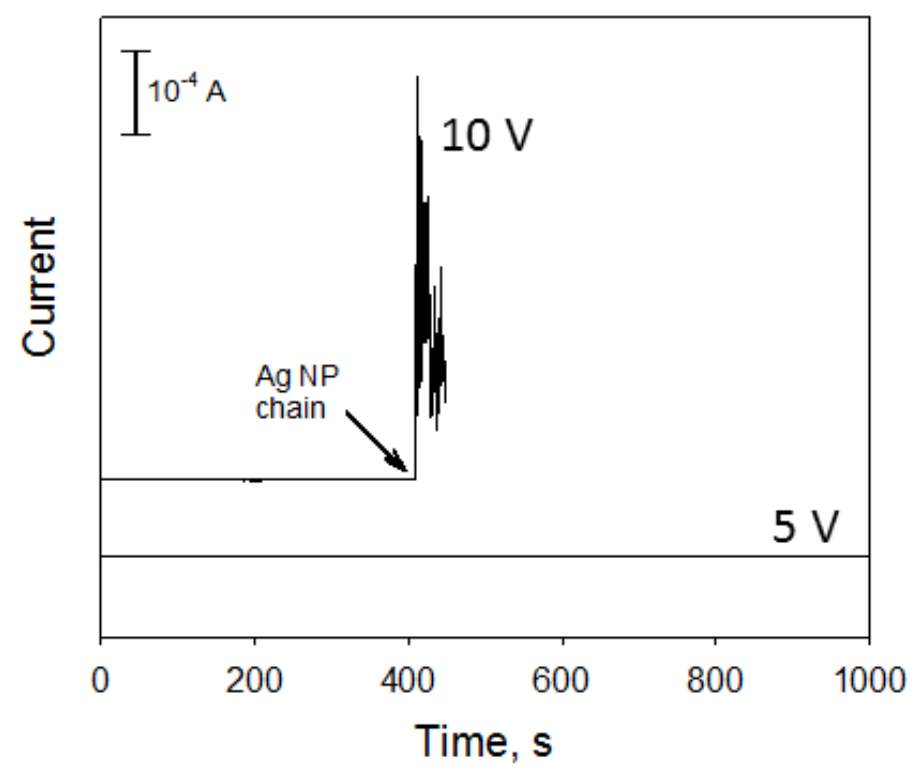

Figure 7.1. I-t plot showing chain formation at the indicated voltage. 
to the same device. The current increased to the $10^{-4} \mathrm{~A}$ level around $400 \mathrm{~s}$ at $10 \mathrm{~V}$, showing spikes. We stopped the application of potential at $450 \mathrm{~s}$. The rise in current indicates Ag NP chains formation. After chain formation, we soaked the device in $5 \mathrm{mM}$ 4-ATP solution in ethanol for $20 \mathrm{~min}$. Later, we rinsed the device with ethanol and nanopure water and dried under $\mathrm{N}_{2}$. After drying, we performed SERS measurements. A $638 \mathrm{~nm}$ laser with a power density of $2.5 \mathrm{~W} / \mathrm{cm}^{2}$ and $40 \mathrm{x}$ lens objective was used for SERS measurements. Figure 7.2 shows an optical image of the device after the chain formation and SERS spectra of the device. The spectrum displayed Raman peaks for 4,4'-DMAB (oxidation of 4-ATP in presence of laser) at $1070 \mathrm{~cm}^{-1}\left(v_{\mathrm{CC}}+v_{\mathrm{CS}}\right), 1180 \mathrm{~cm}^{-1}\left(\beta_{\mathrm{CH}}\right), 1140 \mathrm{~cm}^{-1}\left(v_{\mathrm{CN}}+\beta_{\mathrm{CH}}\right), 1384$ $\mathrm{cm}^{-1}\left(v_{\mathrm{CN}}+v_{\mathrm{NN}}\right), 1440 \mathrm{~cm}^{-1}\left(v_{\mathrm{NN}}+\beta_{\mathrm{CH}}\right)$, and $1575 \mathrm{~cm}^{-1}\left(v_{\mathrm{CC}}\right) .{ }^{248,249}$ SERS mapping was performed over a $0.8 \times 0.8 \mu \mathrm{m}^{2}$ area by recording a Raman spectrum with a $0.5 \mathrm{~s}$ acquisition time at the microgap region. Figure 7.3 shows the Raman map and I-t plot of the device. We carried out Raman measurements of the 4-ATP coated on the device while the device was made to switch from HRS to LRS and vice-versa. Figure 7.3 A shows the Raman map and Figure 7.3 B shows the I-t plot of the device carried out simultaneously with Raman mapping. The spectral map shows the high and low intensity regions of the Ag NP chain. This is due to high density of Ag at the positive side of the electrode where the NP chain formation takes place and low density of $\mathrm{Ag}$ at the bare Au side of the electrode. As seen in Chapter V, NP chain formation exhibits a cone-shape structure growing from positive side towards negative side. 


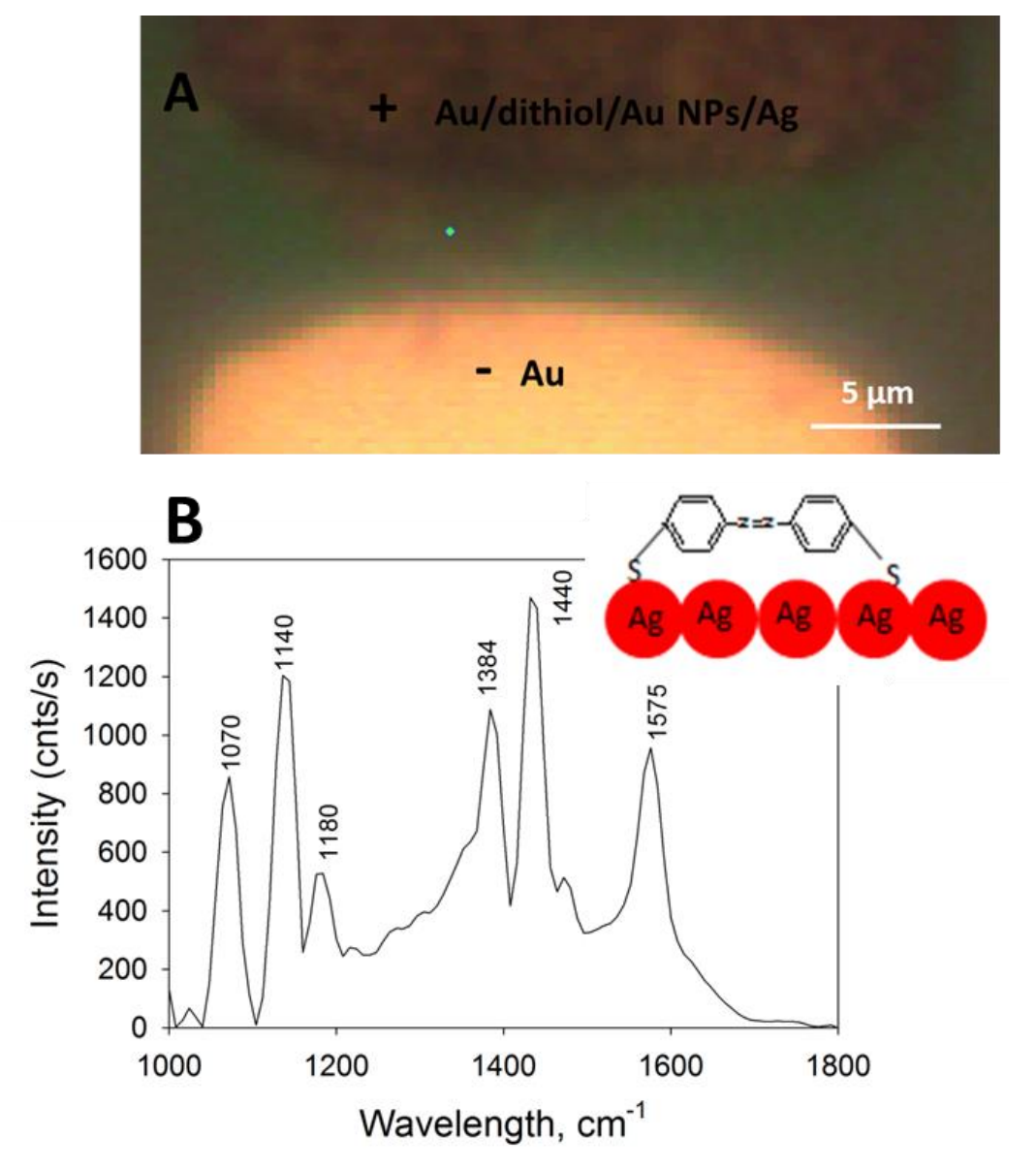

Figure 7.2. (A) Optical image of the device after Ag NP chain formation and (B) SERS spectra of DMAB molecule at the chain region without any application of potential. 



Figure 7.3. (A) SERS mapping at the chain region showing the intensity map and (B) I-t plot of the device for pulse width $30 \mathrm{~s}$ at $-0.5 \mathrm{~V}$ and $-5 \mathrm{~V}$ carried out simultaneously with Raman mapping. 
Further, SERS signal for aggregated nanoparticles is strong compared to isolated nanoparticle due to hot spots generated from the coupled nanoparticles. Chen et al studied the SERS in the hotspots of coupled dimers and trimers and observed that Raman intensity increased with the increase in number of nanoparticles. ${ }^{251}$

Figure 7.4 A displays a potential step current (I) - time (t) plot of the $\mathrm{Au} / \mathrm{Ag} \mathrm{NP}$ chain/Au junction along with the SERS spectra recorded at $-0.5 \mathrm{~V}$ and $5 \mathrm{~V}$. The I-t curve was obtained by stepping the voltage between $-0.5 \mathrm{~V}$ and $-5 \mathrm{~V}$ with a $30 \mathrm{~s}$ holding time at each voltage. A Raman spectrum was recorded at each potential. The current-time plot shows the distinct ON and OFF states with resistance ratio of 100. Figure 7.4 B shows the SERS spectra of the device while it was switched between ON and OFF states. The spectrum shows both ON and OFF states of the region marked in Figure 7.4 A in the Raman map region shown as rectangle and labelled as a, b, c, d, and e. We carried out Raman mapping at the microgap region where Ag NP chain is formed and selected high intensity region among the map for the Ag NP chain. The SERS spectra of these 5 squares are marked as a, b, c, d, and e showing both ON and OFF states for each square. The on (red) and off (black) states are shown as overlay marked as a, b, c, d, and e in Figure 7.4 B. The spectra shows significant enhancement of all peaks in the OFF state which is due to electrons localized at one point, while in the ON state electrons are moving from one position to another and thus non-localized giving lower intensity. In Figure 7.4 B, spectra 'a' show the ON and OFF spectra of the top most intense white square in the rectangular region showing high intensity. 
B
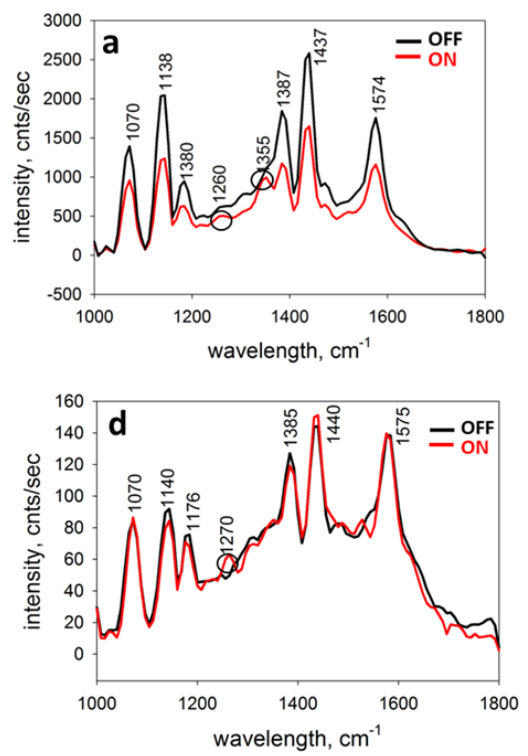
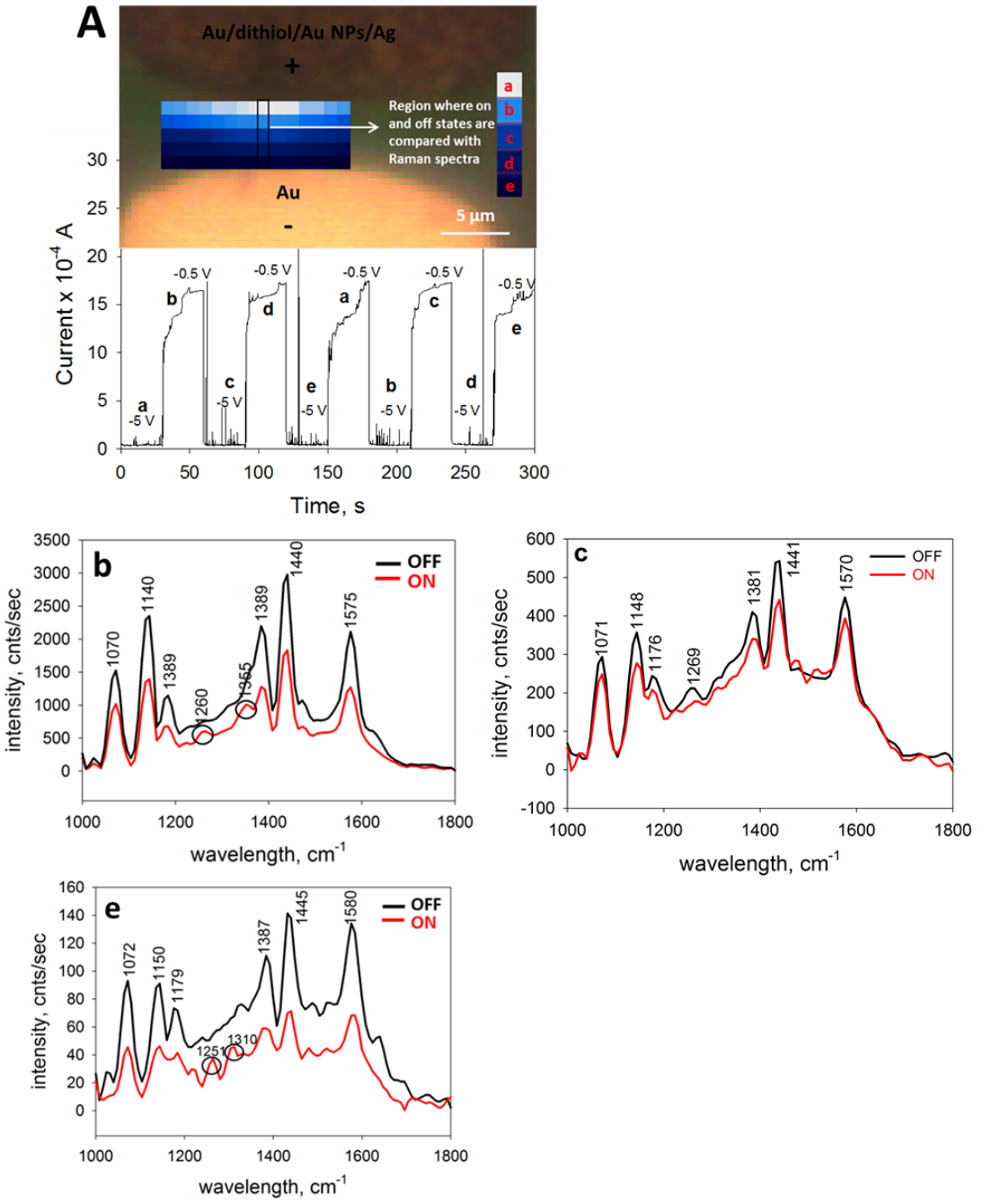

Figure 7.4. (A) Current-time plot and Raman map of the device at the Ag NP chain region. The rectangular region in the Raman map labelled as a, b, c, d, and e further correlates to the on and off state in the current-time plot and (B) SERS spectra of the DMAB molecule showing overlay of on and off state for regions labelled a,b,c,d,and e. 
Similarly spectra ' $b$ ' for the next square and so on. From these results, we conclude that most changes are observed at the region near to the positive side of the electrode. The encircled peak at wavelength range of $1260-1270 \mathrm{~cm}^{-1}$ at the ON state is assigned to $v_{\mathrm{CC}}+\delta_{\mathrm{CH}}$ modes of trans-DMAB. ${ }^{249,250}$ The other enhanced peak observed at for spectra ' $a$ ' and ' $b$ ' is unknown but it shows significant difference in the ON and OFF state of the device. Further it is not seen with other spectra. This may be because the voltage applied creates significant changes at the upper region of the chain. The SERS spectra taken at the two different applied voltages are similar to those obtained without the external field, indicating the molecular layer is intact under the applied voltages. Figure 7.5 shows the SEM image of the device after SERS spectra. For better explanation, we placed the map region and SEM image side by side. The mapping region resembles the SEM image by showing a cone shape pattern. From the SEM, we conclude that the SERS spectra and conductance switching showed maximum effect at the cone region of the chain.

\section{Surface plasmon properties of 1D Ag nanochains.}

We fabricated Au/dithiol/Au NPs with Ag electrodeposited in the presence of $\mathrm{CTAB}$ on one side of the device. After Ag deposition, we applied voltages to form the chains. Figure 7.6 shows the current-time plot of the device where chains formed at $5 \mathrm{~V}$. After the formation of chains, we took the dark-field microscopy image of the device using a 100x oil immersion lens. We carried out LSPR 

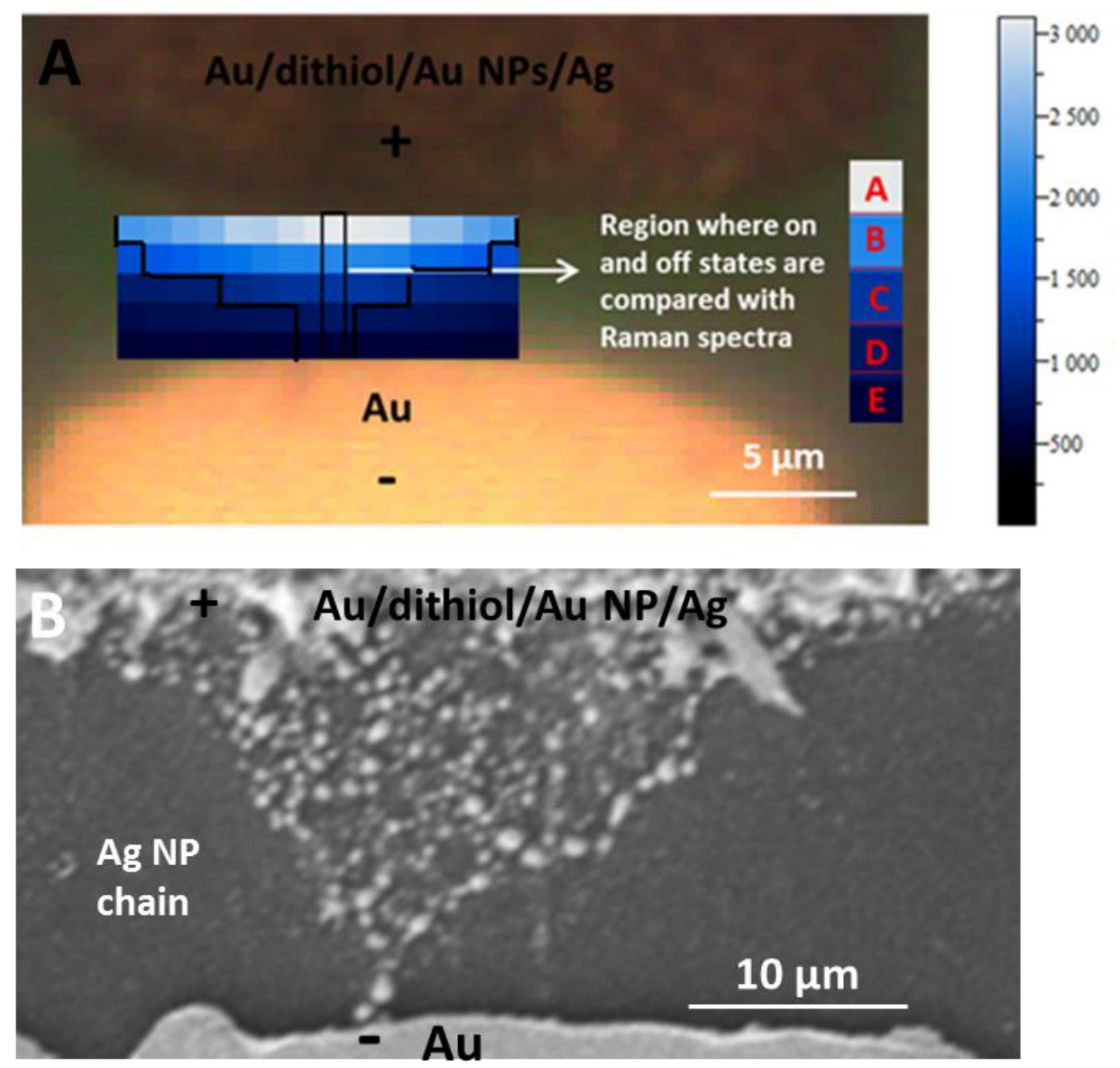

Figure 7.5. (A) Raman map showing cone shape pattern drawn on the basis of intensity of each squares, and (B) SEM image of the same device showing cone shape structure of the Ag NP chain. 
measurements from $450 \mathrm{~nm}$ to $900 \mathrm{~nm}$. Figure 7.6 shows the dark field image after chain formation and LSPR measurement with and without normalization of the peak intensity. Figure 7.7 A shows the dark field image of the Au/TED device. It also shows the different regions from where spectra were taken. Figure 7.7 B shows the overlay of all the Vis/NIR spectra at different regions. Because of the asymmetry structure, coupling along the long axis direction is significantly stronger than that along the short-axis direction. The spectrum under long-axis polarization exhibits at least two modes, including a strong highly red-shifted mode centered at around $830 \mathrm{~nm}$ and a weaker mode around $600 \mathrm{~nm}$. The mode around $830 \mathrm{~nm}$ is called the dipolar mode $\mathrm{e}^{157,158}$ and the mode around $600 \mathrm{~nm}$ is thought to be a quadrupole mode. ${ }^{158}$

\subsection{Conclusions}

We demonstrated simultaneous conductivity and SERS measurements at electrochemically-fabricated Ag NP chain junctions coated with 4-ATP. Our data showed dramatic changes for the SERS peaks of DMAB/Ag NP chain. The OFF state showed significant enhancements of all peaks with increased intensity compared to ON state showing lower intensity. The peak enhancement at wavelength $1270 \mathrm{~cm}^{-1}$ was consistent which we attribute to $v_{\mathrm{CC}}$ in vibration mode in phenyl ring for trans-DMAB based on literature. The simultaneous recorded Raman spectra clearly say that the molecular structure is intact. Our method could be useful to see how conductivity can affect SERS enhancement. 


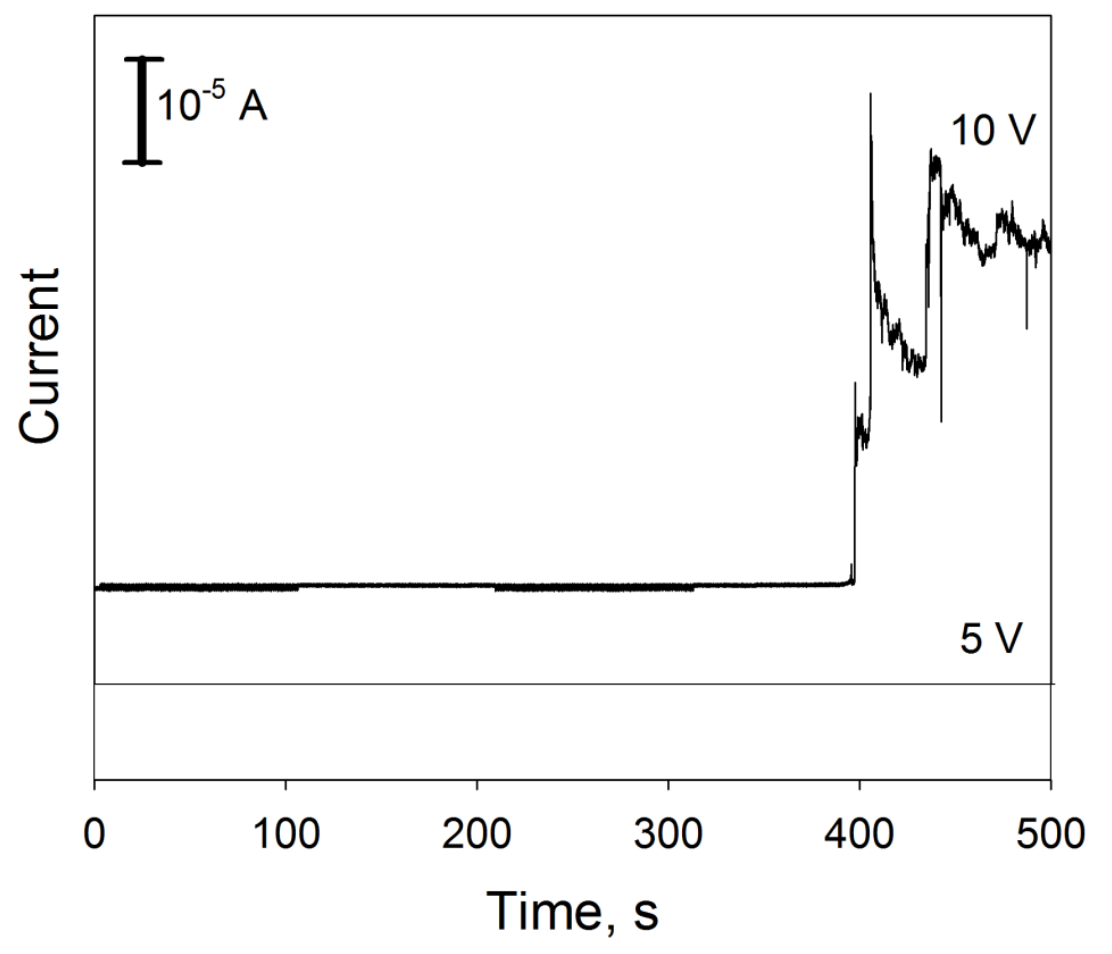

Figure 7.6. Current-time plot of the sample showing chain formation at the indicated voltage. 

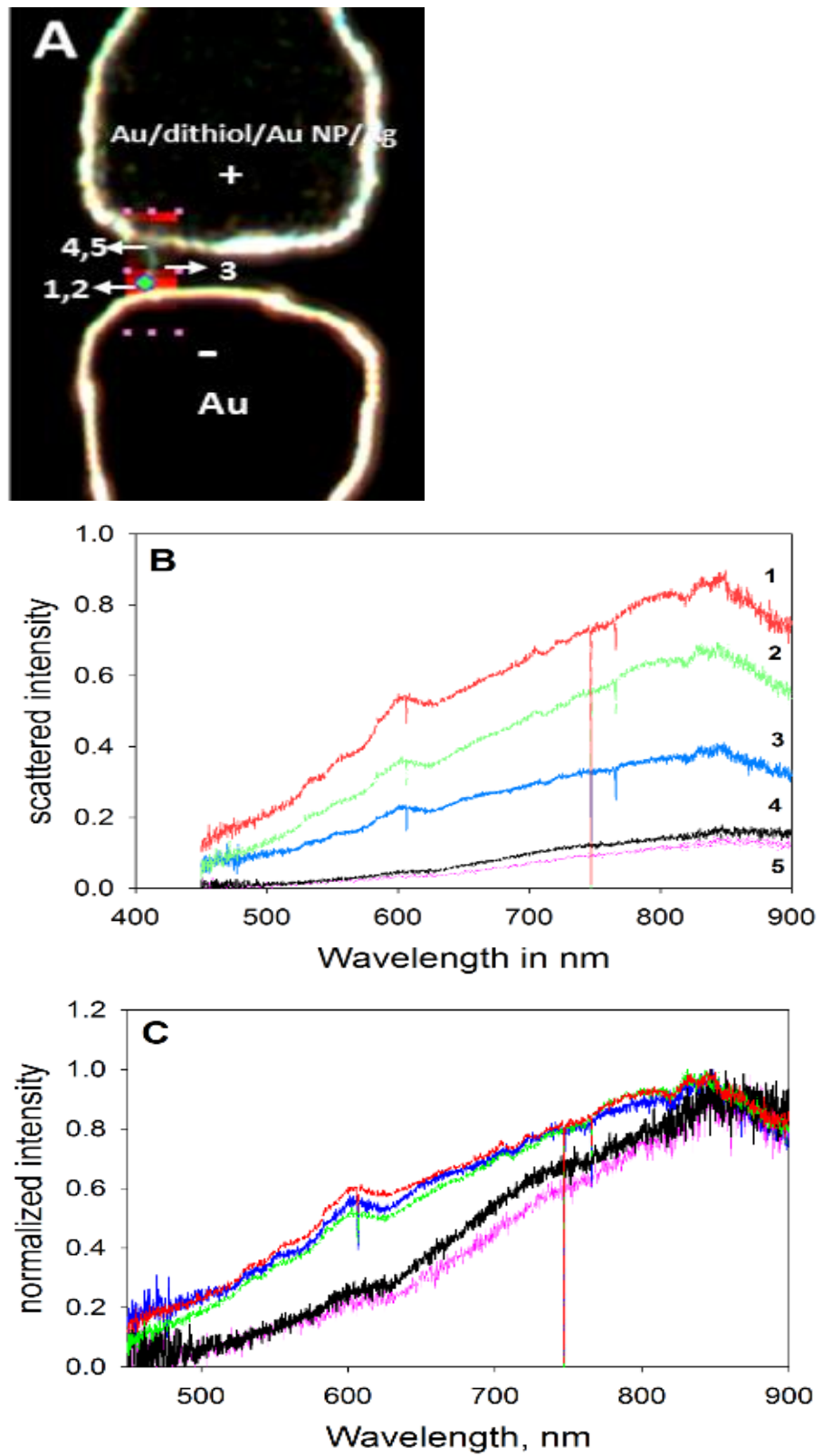

Figure 7.7. (A) Dark-field image of the Au/Ag NP chain/Au device, (B) Darkfield light scattering spectra at different locations in the 1D chain and (C) Normalized spectra of (B). 
LSPR studies open up another field to study the plasmonic properties of 1D assemblies at the microgap junctions. The optical response of $1 \mathrm{D}$ assemblies having asymmetric distribution shows red-shifted spectrum towards NIR as compared to isolated nanoparticles. Future work will be directed towards the simultaneous conductivity and LSPR measurements. 


\section{CHAPTER VIII}

\section{SUMMARY AND FUTURE DIRECTIONS}

\subsection{Summary}

This dissertation described an electrochemical approach to form metal/nanoparticle chain/metal and metal/nanowire/polyphenol/metal junctions at 5 micron gap Au electrodes and to form Ag NRs/NWs directly on to glass/ITO electrode surfaces using an electrochemical seed-mediated growth approach. The approach is simple, fast and does not require any template to form the $1 \mathrm{D}$ assembly at 5 micron gap electrodes. 1D assembly prepared in different ways shows several potential applications in nanoelectronics, sensing and plasmonics.

We successfully fabricated the following nanoscale junctions combining electrochemistry and self-assembly: (Electrode 1, Electrode 2, nanoparticle chain, nanorod and nanowire are abbreviated as E1, E2, NP chain, NR and NW, respectively)

1) $\mathrm{E} 1 / \mathrm{Ag} \mathrm{NP}$ chain/E2

2) $\mathrm{E} 1 / \mathrm{Ag} \mathrm{NW} /$ polyphenol/E2

3) Glass/ITO/Ag NRs/NWs 
The mechanism of formation of one dimensional nanochains is electrochemical in nature where metal gets oxidized at the cathode and reduced at the anode under the applied potential. The approach is simple and does not require any template or aqueous media to form the chains across the gap. 1D nanochains formed across microgap electrodes show interesting resistive switching properties. The resistive switching could be bipolar or unipolar. Our devices showed unipolar behavior for most of the cases. The following conclusions were drawn from these studies

- E1/Ag NP chain/E2 junctions can be formed in air in the presence of humidity (above 20\%) and surfactant at different voltages of 0-10 V. The voltage depends on gap, amount of Ag, amount of surfactant and humidity level. Ag nanoparticle chain formation was carried out with Ag electrodeposited one side or two sides of the device. Ag NP chain on one side was formed at higher negative potentials $(10 \mathrm{~V})$ compared to two sides $(5 \mathrm{~V})$ this might be due to Ag contribution from both sides helping in making connection at early potentials.

- E1/Ag NP chain/E2 junctions can be made to switch from $0.5 \mathrm{~V}$ to $5 \mathrm{~V}$ showing ON state at $0.5 \mathrm{~V}$ and OFF state at $5 \mathrm{~V}$.

- The numbers of chains formed during the initial formation step remain the same even after performing several switching cycles. No new chain was observed during switching operations. This says that the resistive switching mechanism might be occurring at atomic scale where device behaves as an atomic switch. 
Electrochemically fabricated metal/Ag NP chain/metal junctions were used for simultaneously measuring the conductivity and SERS of E1/Ag NP chain/E2 junctions coated with 4-ATP. The conclusions drawn from this study are as follows:

- The observed Raman changes for oxidized-ATP in the high and low conductance state was likely due to chemical or structural changes inside the Ag nanoparticle chain or from current.

- Significant changes in the Raman modes were observed when the potential was switched from $0.5 \mathrm{~V}$ to $5 \mathrm{~V}$. This is still unclear.

Electrochemical fabrication of metal/Ag NW/polyphenol/metal device.

- E1/Ag NW/polyphenol/E2 devices showed switching behavior where by changing voltage from $0.5 \mathrm{~V}$ to $5 \mathrm{~V}$ showing $\mathrm{ON}$ state at $0.5 \mathrm{~V}$ and $\mathrm{OFF}$ state at $5 \mathrm{~V}$.

- The device showed switching ratio $>100$ and endurance behavior of at least 1000 cycles.

- The SEM image of the device after the switching operations showed that the Ag NW transformed into small Ag NP chains. These Ag NP chains appear to be responsible for the switching behavior. It is possible to use the Ag NP chains for switching polyphenol on the bare side of the device.

- The presence of the polyphenol film showed increase in the switching ratio and improved stability of the device. 
Finally the two terminal resistance switches formed by the devices E1/Ag NP chain/E2 and E1/Ag NW/polypheno1/E2 showed good switching behavior with $\mathrm{ON} / \mathrm{OFF}$ ratio $\geq 10$ and endurance cycle $>1000$ cycles.

This dissertation demonstrates that the combination of electrochemistry, selfassembly, and microfabrication is a powerful tool for the simple, low cost and rapid fabrication of nano devices on a bench top that is useful in electronics or sensing. Improvement is still needed to develop single nanoparticle chain device which would further help in performing LSPR and Raman studies. The work described here offers exploration of several exciting future studies.

\subsection{Future directions}

Further improvements in formation of single nanoparticle chain with the idea of improving chain morphology across microgap electrodes could be beneficial in performing SERS and LSPR sensing. This improvement could be done in several ways: one way is to use patterned substrates; the other way is to use large gap size electrodes or produce natural channels which will form the pathway for nanoparticle chains across the gap. One of the methods that we tried to grow chains is by using large gap size electrodes. The two electrodes with gaps of 10 $\mu \mathrm{m}$ and $23 \mu \mathrm{m}$ were used in our study. We found issues with this study that needs to be addressed. These issues are reproducibility and chain morphology. They can be addressed by carrying out a thorough study by evaluating all the parameters responsible for the formation of nanoparticle chains. Some of the parameters that can be altered are humidity, surfactant concentration and Ag concentration on the 
electrodes, may lead to improvement in the quality of the Ag chains across the electrodes. Further with these improvements, it would be very interesting to study the SERS and LSPR properties of the chains at conductive and insulating states. 


\section{REFERENCES}

(1) Xia, Y.; Yang, P.; Sun, Y.; Wu, Y.; Mayers, B.; Gates, B.; Yin, Y.; Kim, F.; Yan, H. Adv. Mater. 2003, 15, 353-389.

(2) Ko, S. H.; Park, I.; Pan, H.; Grigoropoulos, C. P.; Pisano, A. P.; Luscombe, C. K.; Fréchet, J. M. J. Nano Lett 2007, 7, 1869-1877.

(3) Ozbay, E. Science 2006, 311, 189-193.

(4) Maier, S. A.; Atwater, H. A. J. Appl. Phys. 2005, 98, 011101.

(5) Esteban, R.; Taylor, R. W.; Baumberg, J. J.; Aizpurua, J. Langmuir 2012, 28, 8881-8890.

(6) Zohar, N.; Chuntonov, L.; Haran, G. J. Photochem. Photobiol., C 2014, 21, 26-39.

(7) Hu, J.; Odom, T. W.; Lieber, C. M. Acc. Chem. Res. 1999, 32, 435-445.

(8) Wang, Z. L. Adv.Mater. 2000, 12, 1295-1298.

(9) Thanh, N. T. K.; Maclean, N.; Mahiddine, S. Chem.Rev. 2014, 114, 7610-7630.

(10) Sajanlal, P. R.; Sreeprasad, T. S.; Samal, A. K.; Pradeep, T. Nano.Rev. 2011.

(11) Leontidis, E.; Kleitou, K.; Kyprianidou-Leodidou, T.; Bekiari, V.; Lianos, P. Langmuir 2002, 18, 3659-3668.

(12) Walter, E. C.; Zach, M. P.; Favier, F.; Murray, B. J.; Inazu, K.; Hemminger, J. C.; Penner, R. M. Chem.Phys.Chem. 2003, 4, 131-138.

(13) Martin, C. R. Science 1994, 266, 1961-1966.

(14) C. Hulteen, J.; Martin, C. R. J.Mater.Chem. 1997, 7, 1075-1087.

(15) Martin, B. R.; Dermody, D. J.; Reiss, B. D.; Fang, M.; Lyon, L. A.; Natan, M. J.; Mallouk, T. E. Adv. Mater. 1999, 11, 1021-1025.

(16) Zhang, Y.; Dai, H. Appl.Phys.Lett. 2000, 77, 3015-3017.

(17) Viau, G.; Fiévet-Vincent, F.; Fiévet, F. Solid State lonics 1996, 84, 259-270.

(18) Wiley, B. J.; Im, S. H.; Li, Z.-Y.; McLellan, J.; Siekkinen, A.; Xia, Y. The Journal of Physical Chemistry B 2006, 110, 15666-15675. 1739.

(19) Wiley, B.; Herricks, T.; Sun, Y.; Xia, Y. Nano Letters 2004, 4, 1733- 
(20) Sun, Y.; Mayers, B.; Xia, Y. Nano Letters 2003, 3, 675-679.

(21) Jana, N. R.; Gearheart, L.; Murphy, C. J. Chem.Mater. 2001, 13, 2313-2322.

(22) Jana, N. R.; Gearheart, L.; Murphy, C. J. J.Phys.Chem.B 2001, 105, 4065-4067.

(23) Jana, N. R.; Gearheart, L.; Murphy, C. J. Chem.Comm. 2001, 617-618.

(24) Liu, C. M.; Guo, L.; Xu, H. B.; Wu, Z. Y.; Weber, J. Microelectron. Eng. 2003, 66, 107-114.

(25) Taub, N.; Krichevski, O.; Markovich, G. J.Phys.Chem.B 2003, 107, 11579-11582.

(26) Wei, Z.; Mieszawska, A. J.; Zamborini, F. P. Langmuir 2004, 20, 43224326.

(27) Mieszawska, A. J.; Zamborini, F. P. Chem. Mater. 2005, 17, 34153420.

(28) Mieszawska, A. J.; Jalilian, R.; Sumanasekera, G. U.; Zamborini, F. P. Journal of the American Chemical Society 2005, 127, 10822-10823.

(29) Abdelmoti, L. G.; Zamborini, F. P. Langmuir 2010, 26, 13511-13521.

(30) Sławiński, G. W.; Zamborini, F. P. Langmuir 2007, 23, 10357-10365.

(31) Tian, Y.; Liu, H.; Zhao, G.; Tatsuma, T. J.Phys.Chem.B 2006, 110, 23478-23481.

(32) Reetz, M. T.; Helbig, W. J. Am. Chem. Soc. 1994, 116, 7401-7402.

(33) Huang, C.-J.; Chiu, P.-H.; Wang, Y.-H.; Chen, W. R.; Meen, T. H. J.Electrochem.Soc. 2006, 153, D129-D133.

(34) Yu; Chang, S.-S.; Lee, C.-L.; Wang, C. R. C. J.Phys.Chem.B 1997, 101, 6661-6664.

(35) Tian, N.; Zhou, Z.-Y.; Sun, S.-G.; Cui, L.; Ren, B.; Tian, Z.-Q. Chem.Comm. 2006, 4090-4092.

(36) Favier, F.; Walter, E. C.; Zach, M. P.; Benter, T.; Penner, R. M. Science 2001, 293, 2227-2231. 16139.

(37) Dasari, R.; Zamborini, F. P. J. Am. Chem. Soc. 2008, 130, 161387293.

(38) Dasari, R.; Ibañez, F. J.; Zamborini, F. P. Langmuir 2010, 27, 7285-

(39) Zhao, L.; Kelly, K. L.; Schatz, G. C. J.Phys.Chem.B 2003, 107, 73437350 . 
(40) Shah, N.; Zamborini, F. P. ACS Nano 2015, 9, 10278-10286.

(41) Yang, Y.; Shi, J.; Tanaka, T.; Nogami, M. Langmuir 2007, 23, 1204212047.

(42) Tang, Z.; Kotov, N. A. Adv. Mater. 2005, 17, 951-962.

(43) Kitching, H.; Shiers, M. J.; Kenyon, A. J.; Parkin, I. P. J. Mater. Chem. A. 2013, 1, 6985-6999.

(44) Liu, S.; Tang, Z. J. Mater. Chem. 2010, 20, 24-35.

(45) Velev, O. D.; Gupta, S. Adv. Mater. 2009, 21, 1897-1905.

(46) Srivastava, S.; Kotov, N. A. Soft Matter 2009, 5, 1146-1156.

(47) Xu, L.; Ma, W.; Wang, L.; Xu, C.; Kuang, H.; Kotov, N. A. Chem. Soc. Rev. 2013, 42, 3114-3126.

(48) Walter, M. V.; Cheval, N.; Liszka, O.; Malkoch, M.; Fahmi, A. Langmuir 2012, 28, 5947-5955.

(49) Kwiat, M.; Cohen, S.; Pevzner, A.; Patolsky, F. Nano Today 2013, 8, 677-694.

(50) Biswas, A.; Bayer, I. S.; Biris, A. S.; Wang, T.; Dervishi, E.; Faupel, F. Adv. Colloid Interface Sci. 2012, 170, 2-27.

(51) Yamamoto, H.; Ohnuma, A.; Ohtani, B.; Kozawa, T. Microelectron. Eng. 2013, 110, 369-373.

(52) Jiang, L.; Chen, X.; Lu, N.; Chi, L. Acc.Chem.Res. 2014, 47, 3009-3017.

(53) Tong, H. D.; Jansen, H. V.; Gadgil, V. J.; Bostan, C. G.; Berenschot, E.; van Rijn, C. J. M.; Elwenspoek, M. Nano Letters 2004, 4, 283-287.

8729.

(54) Yin, Y.; Lu, Y.; Gates, B.; Xia, Y. J. Am. Chem. Soc. 2001, 123, 8718-

(55) Masuda, Y.; Itoh, M.; Yonezawa, T.; Koumoto, K. Langmuir 2002, 18, 4155-4159.

(56) Cui, Y.; Björk, M. T.; Liddle, J. A.; Sönnichsen, C.; Boussert, B.; Alivisatos, A. P. Nano Lett 2004, 4, 1093-1098.

(57) Bae, C.; Shin, H.; Moon, J. Chem.Mater. 2007, 19, 1531-1533.

(58) Ito, T.; Okazaki, S. Nature 2000, 406, 1027-1031.

(59) Alivisatos, A. P.; Johnsson, K. P.; Peng, X.; Wilson, T. E.; Loweth, C. J.; Bruchez, M. P.; Schultz, P. G. Nature 1996, 382, 609-611.

(60) Le, J. D.; Pinto, Y.; Seeman, N. C.; Musier-Forsyth, K.; Taton, T. A.; Kiehl, R. A. Nano Lett 2004, 4, 2343-2347. 
(61) Fu, X.; Wang, Y.; Huang, L.; Sha, Y.; Gui, L.; Lai, L.; Tang, Y. Adv.Mater. 2003, 15, 902-906.

(62) He, J.; Kunitake, T.; Nakao, A. Chem.Mater. 2003, 15, 4401-4406.

(63) Ellis, A. V.; Vijayamohanan, K.; Goswami, R.; Chakrapani, N.; Ramanathan, L. S.; Ajayan, P. M.; Ramanath, G. Nano Lett. 2003, 3, 279-282.

(64) Liao, J.; Zhang, Y.; Yu, W.; Xu, L.; Ge, C.; Liu, J.; Gu, N. Colloids Surf., A 2003, 223, 177-183.

(65) Sardar, R.; Shumaker-Parry, J. S. Nano Lett. 2008, 8, 731-736.

(66) Sau, T. K.; Murphy, C. J. Langmuir 2005, 21, 2923-2929.

(67) Yong, Y.; Shigemasha, M.; Masayuki, N.; Jianlin, S.; Weiming, H. Nanotechno 2006, 17, 2821.

(68) Thomas, J. R. J.Appl.Phys. 1966, 37, 2914-2915.

(69) Gong, T.; Marr, D. W. M. Appl.Phys.Lett. 2004, 85, 3760-3762.

(70) Klajn, R.; Bishop, K. J. M.; Grzybowski, B. A. Proc. Natl. Acad. Sci. U. S. A. 2007, 104, 10305-10309.

(71) Velev, O. D.; Bhatt, K. H. Soft Matter 2006, 2, 738-750.

(72) Ryan, K. M.; Mastroianni, A.; Stancil, K. A.; Liu, H.; Alivisatos, A. P. Nano Lett. 2006, 6, 1479-1482.

(73) Kuzyk, A. ELECTROPHORESIS 2011, 32, 2307-2313.

(74) Kumar, S.; Yoon, S.-H.; Kim, G.-H. Curr. Appl. Phys. 2009, 9, 101-103.

(75) Barsotti, R. J.; Vahey, M. D.; Wartena, R.; Chiang, Y.-M.; Voldman, J.; Stellacci, F. Small 2007, 3, 488-499.

(76) Hermanson, K. D.; Lumsdon, S. O.; Williams, J. P.; Kaler, E. W.; Velev, O. D. Science 2001, 294, 1082-1086.

(77) Leiterer, C.; Berg, S.; Eskelinen, A.-P.; Csaki, A.; Urban, M.; Törmä, P.; Fritzsche, W. Sens. Actuators, B 2013, 176, 368-373.

(78) Bhatt, K. H.; Velev, O. D. Langmuir 2003, 20, 467-476.

(79) Erez, B.; Yoav, E.; Uri, S.; Gdalyahu, B.-Y. Nature 1998, 391, 775-778.

(80) Keren, K.; Krueger, M.; Gilad, R.; Ben-Yoseph, G.; Sivan, U.; Braun, E. Science 2002, 297, 72-75.

(81) Richter, J.; Seidel, R.; Kirsch, R.; Mertig, M.; Pompe, W.; Plaschke, J.; Schackert, H. K. Adv.Mater. 2000, 12, 507-510.

(82) Kumar, A.; Pattarkine, M.; Bhadbhade, M.; Mandale, A. B.; Ganesh, K. N.; Datar, S. S.; Dharmadhikari, C. V.; Sastry, M. Adv.Mater. 2001, 13, 341-344. 
(83) Ford, W. E.; Harnack, O.; Yasuda, A.; Wessels, J. M. Adv.Mater. 2001, 13, 1793-1797.

(84) Walsh, D.; Arcelli, L.; Ikoma, T.; Tanaka, J.; Mann, S. Nat.Mater. 2003, 2, 386-390.

(85) Cao, Y.; Zhou, Y. M.; Shan, Y.; Ju, H. X.; Xue, X. J. Adv.Mater. 2006, $18,1838-1841$.

(86) Shibu Joseph, S. T.; Ipe, B. I.; Pramod, P.; Thomas, K. G. J. Phys. Chem. B. 2006, 110, 150-157.

(87) Thomas, K. G.; Barazzouk, S.; Ipe, B. I.; Joseph, S. T. S.; Kamat, P. V. J. Phys. Chem. B. 2004, 108, 13066-13068.

(88) Sudeep, P. K.; Joseph, S. T. S.; Thomas, K. G. J. Am.Chem.Soc. 2005, $127,6516-6517$.

(89) Nie, Z.; Fava, D.; Kumacheva, E.; Zou, S.; Walker, G. C.; Rubinstein, M. Nat.Mater. 2007, 6, 609-614.

(90) Quintana, M.; Ke, X.; Van Tendeloo, G.; Meneghetti, M.;

Bittencourt, C.; Prato, M. ACS Nano 2010, 4, 6105-6113.

(91) Sun, Y.-P.; Huang, W.; Lin, Y.; Fu, K.; Kitaygorodskiy, A.; Riddle, L. A.; Yu, Y. J.; Carroll, D. L. Chem.Mater. 2001, 13, 2864-2869.

(92) Ang, L.-M.; Hor, T. S. A.; Xu, G.-Q.; Tung, C.-h.; Zhao, S.; Wang, J. L. S. Chem.Mater. 1999, 11, 2115-2118.

(93) Hrapovic, S.; Liu, Y.; Male, K. B.; Luong, J. H. T. Anal. Chem. 2004, 76, 1083-1088.

(94) Hutchinson, T. O.; Liu, Y. P.; Kiely, C.; Kiely, C. J.; Brust, M. Adv.Mater. 2001, 13, 1800-1803.

(95) Mieszawska, A. J.; Slawinski, G. W.; Zamborini, F. P. J. Am.Chem.Soc. 2006, 128, 5622-5623.

(96) Zhang, X.; Imae, T. J. Phys. Chem. C. 2009, 113, 5947-5951.

(97) Li, C. Z.; He, H. X.; Bogozi, A.; Bunch, J. S.; Tao, N. J. Appl.Phys.Lett. 2000, 76, 1333-1335.

(98) Cheng, C.; Gonela, R. K.; Gu, Q.; Haynie, D. T. Nano Lett. 2005, 5, 175-178.

(99) Sun, H.; Liu, Q.; Li, C.; Long, S.; Lv, H.; Bi, C.; Huo, Z.; Li, L.; Liu, M. Adv. Funct. Mater. 2014, 24, 5679-5686.

(100) Liu, Q.; Sun, J.; Lv, H.; Long, S.; Yin, K.; Wan, N.; Li, Y.; Sun, L.; Liu, M. Adv. Mater. 2012, 24, 1844-1849. 
(101) Perez, T.; De Rose, C. A. 2010.

(102) Han, S.-T.; Zhou, Y.; Roy, V. A. L. Adv.Mater. 2013, 25, 5425-5449.

(103) Waser, R.; Aono, M. Nat. Mater. 2007, 6, 833-840.

(104) Kim, K. M.; Jeong, D. S.; Hwang, C. S. Nanotechnol 2011, 22, 254002/254001-254002/254017.

(105) Jeong, D. S.; Thomas, R.; Katiyar, R. S.; Scott, J. F.; Kohlstedt, H.; Petraru, A.; Hwang, C. S. Rep. Prog. Phys. 2012, 75, 076502/076501076502/076531.

(106) Makarov, A.; Sverdlov, V.; Selberherr, S. Microelectron. Reliab. 2012, $52,628-634$.

(107) Li, Y.; Long, S.; Liu, Q.; Lü, H.; Liu, S.; Liu, M. Chin. Sci. Bull. 2011, 56, 3072-3078.

(108) An, C.; Haddad, S.; Yi-Ching, W.; Tzu-Ning, F.; Zhida, L.; Avanzino, S.; Pangrle, S.; Buynoski, M.; Rathor, M.; Wei, C.; Tripsas, N.; Bill, C.; VanBuskirk, M.; Taguchi, M. In Electron Devices Meeting, 2005. IEDM Technical Digest. IEEE International 2005, p 746-749.

(109) Scott, J. F.; Paz de Araujo, C. A. Science 1989, 246, 1400-1405.

(110) Waser, R.; Dittmann, R.; Staikov, G.; Szot, K. Adv. Mater. 2009, 21, 2632-2663.

(111) Beck, A.; Bednorz, J. G.; Gerber, C.; Rossel, C.; Widmer, D. Appl.Phys.Lett. 2000, 77, 139-141.

(112) Liu, S. Q.; Wu, N. J.; Ignatiev, A. Appl.Phys.Lett. 2000, 76, 2749-2751.

(113) Fontana, R. E.; Hetzler, S. R. J. Appl. Phys. 2006, 99, 08 N902.

(114) Wuttig, M.; Yamada, N. Nat.Mater. 2007, 6, 824-832.

(115) Kozicki, M.; Yun, M.; Hilt, L.; Singh, A. Pennington NJ USA:

Electrochem. Soc 1999, 298-309.

(116) Jin, Z.; Liu, G.; Wang, J. J. Mater. Chem. C 2013, 1, 3282-3286.

(117) Rosezin, R.; Meier, M.; Breuer, U.; Ku; x; geler, C.; Waser, R. IEEE Trans. Nanotechnol. 2011, 10, 338-343.

(118) Liao, Z.-M.; Hou, C.; Zhao, Q.; Wang, D.-S.; Li, Y.-D.; Yu, D.-P. Small 2009, 5, 2377-2381.

(119) Kim, D. C.; Seo, S.; Ahn, S. E.; Suh, D.-S.; Lee, M. J.; Park, B.-H.; Yoo, I. K.; Baek, I. G.; Kim, H.-J.; Yim, E. K.; Lee, J. E.; Park, S. O.; Kim, H. S.; Chung, U.-I.; Moon, J. T.; Ryu, B. I. Appl.Phys.Lett. 2006, 88, 202102. 
(120) Choi, B. J.; Jeong, D. S.; Kim, S. K.; Rohde, C.; Choi, S.; Oh, J. H.; Kim, H. J.; Hwang, C. S.; Szot, K.; Waser, R.; Reichenberg, B.; Tiedke, S. J. Appl. Phys. 2005, 98, 033715.

(121) Waser, R.; Baiatu, T.; Härdtl, K.-H. J. Am. Ceram. Soc. 1990, 73, 1645-1653.

(122) Yang, Y.; Gao, P.; Gaba, S.; Chang, T.; Pan, X.; Lu, W. Nat. Commun. 2012, 3, 1737/1731-1737/1738.

(123) Gao, S.; Song, C.; Chen, C.; Zeng, F.; Pan, F. Appl. Phys. Lett. 2013, $102,141606 / 141601-141606 / 141605$.

(124) Celano, U.; Goux, L.; Belmonte, A.; Opsomer, K.; Franquet, A.; Schulze, A.; Detavernier, C.; Richard, O.; Bender, H.; Jurczak, M.; Vandervorst, W. Nano Lett. 2014, 14, 2401-2406.

(125) Aono, M.; Hasegawa, T. Proceedings of the IEEE 2010, 98, 22282236.

(126) SchirmC; MattM; PaulyF; Cuevas, J. C.; NielabaP; ScheerE Nat. Nano. 2013, 8, 645-648.

(127) Kundu, M.; Terabe, K.; Hasegawa, T.; Aono, M. J. Appl. Phys. 2006, 99, 103501.

(128) Kundu, M.; Hasegawa, T.; Terabe, K.; Aono, M. J. Appl. Phys. 2008, 103, 073523.

(129) Soni, R.; Meuffels, P.; Petraru, A.; Weides, M.; Kügeler, C.; Waser, R.; Kohlstedt, H. J. Appl. Phys. 2010, 107, 024517.

(130) Tsunoda, K.; Fukuzumi, Y.; Jameson, J. R.; Wang, Z.; Griffin, P. B.; Nishi, Y. Appl.Phys.Lett. 2007, 90, 113501.

(131) Yang, Y.; Gao, P.; Gaba, S.; Chang, T.; Pan, X.; Lu, W. Nat.Commun. 2012, 3, 732.

(132) Guo, X.; Schindler, C.; Menzel, S.; Waser, R. Appl. Phys. Lett. 2007, 91, 133513/133511-133513/133513.

(133) He, C.; Shi, Z.; Zhang, L.; Yang, W.; Yang, R.; Shi, D.; Zhang, G. ACS Nano 2012, 6, 4214-4221.

(134) Mutiso, R. M.; Kikkawa, J. M.; Winey, K. I. Appl.Phys.Lett. 2013, 103,

(135) Waser, R. Microelectron.Eng. 2009, 86, 1925-1928.

(136) Strukov, D. B.; Kohlstedt, H. MRS Bull. 2012, 37, 108-114.

(137) Waser, R. Wiley-VCH 2003, 527. 
(138) White, S. I.; Vora, P. M.; Kikkawa, J. M.; Winey, K. I. Adv. Funct. Mater. 2011, 21, 233-240.

(139) Kozicki, M. N.; Mira, P.; Mitkova, M. IEEE Trans. Nanotechnol. 2005, 4, 331-338.

(140) Liang, C.; Terabe, K.; Tsuruoka, T.; Osada, M.; Hasegawa, T.; Aono, M. Adv. Funct.Mater. 2007, 17, 1466-1472.

(141) Schindler, C.; Weides, M.; Kozicki, M. N.; Waser, R. Appl. Phys. Lett. 2008, 92, 122910/122911-122910/122913.

(142) Schindler, C.; Staikov, G.; Waser, R. Appl.Phys.Lett. 2009, 94, 072109.

(143) Jafar, M.; Haneman, D. Phys.Rev.B. 1994, 49, 13611-13615.

(144) Jo, S. H.; Lu, W. Nano Lett. 2008, 8, 392-397.

(145) Jo, S. H.; Kim, K.-H.; Lu, W. Nano Lett. 2009, 9, 496-500.

(146) Tian, H.; Chen, H.-Y.; Gao, B.; Yu, S.; Liang, J.; Yang, Y.; Xie, D.; Kang, J.; Ren, T.-L.; Zhang, Y.; Wong, H. S. P. Nano Lett. 2013, 13, 651-657.

(147) Shoute, L. C. T.; Wu, Y.; McCreery, R. L. Electrochim. Acta 2013, 110, 437-445.

(148) Rasskazov, I. L.; Karpov, S. V.; Markel, V. A. Phys.Rev.B. 2014, 90, 075405.

(149) Krenn, J. R.; Dereux, A.; Weeber, J. C.; Bourillot, E.; Lacroute, Y.; Goudonnet, J. P.; Schider, G.; Gotschy, W.; Leitner, A.; Aussenegg, F. R.; Girard, C. Phys.Rev.Lett. 1999, 82, 2590-2593. 1716.

(150) Maier, S. A.; Kik, P. G.; Atwater, H. A. Appl.Phys.Lett. 2002, 81, 1714-

(151) Maier, S. A.; Brongersma, M. L.; Kik, P. G.; Atwater, H. A. Phys.Rev.B. 2002, 65, 193408.

(152) Weber, W. H.; Ford, G. W. Phys.Rev.B. 2004, 70, 125429.

(153) Alù, A.; Engheta, N. Phys.Rev.B. 2006, 74, 205436.

(154) Quinten, M.; Leitner, A.; Krenn, J. R.; Aussenegg, F. R. Opt. Lett. 1998, 23, 1331-1333.

(155) Harris, N.; Arnold, M. D.; Blaber, M. G.; Ford, M. J. J. Phys. Chem. C. 2009, 113, 2784-2791.

(156) Lévêque, G.; Quidant, R. Opt. Express 2008, 16, 22029-22038.

(157) Barrow, S. J.; Funston, A. M.; Gómez, D. E.; Davis, T. J.; Mulvaney, P. Nano Lett. 2011, 11, 4180-4187. 
(158) Hanske, C.; Tebbe, M.; Kuttner, C.; Bieber, V.; Tsukruk, V. V.; Chanana, M.; König, T. A. F.; Fery, A. Nano Lett. 2014, 14, 6863-6871.

(159) Jana, N. R.; Gearheart, L.; Murphy, C. J. J. Phys. Chem. C 2001, 105, 4065-4067.

(160) Gole, A.; Murphy, C. J. Chem. Mater. 2004, 16, 3633-3640.

(161) Huang, Y.; Duan, X.; Wei, Q.; Lieber, C. M. Science 2001, 291, 630633.

(162) Smith, P. A.; Nordquist, C. D.; Jackson, T. N.; Mayer, T. S.; Martin, B. R.; Mbindyo, J.; Mallouk, T. E. Appl.Phys.Lett. 2000, 77, 1399-1401.

(163) Cui, Y.; Wei, Q.; Park, H.; Lieber, C. M. Science 2001, 293, 12891292.

(164) Kempa, K.; Kimball, B.; Rybczynski, J.; Huang, Z. P.; Wu, P. F.; Steeves, D.; Sennett, M.; Giersig, M.; Rao, D. V. G. L. N.; Carnahan, D. L.; Wang, D. Z.; Lao, J. Y.; Li, W. Z.; Ren, Z. F. Nano Lett. 2003, 3, 13-18.

(165) Maier, S. A.; Kik, P. G.; Atwater, H. A.; Meltzer, S.; Harel, E.; Koel, B. E.; Requicha, A. A. G. Nat.Mater. 2003, 2, 229-232. 1259.

(166) Whang, D.; Jin, S.; Wu, Y.; Lieber, C. M. Nano Lett. 2003, 3, 1255-

(167) Sau, T. K.; Murphy, C. J. Langmuir 2004, 20, 6414-6420.

(168) Johnson, C. J.; Dujardin, E.; Davis, S. A.; Murphy, C. J.; Mann, S. J. Mater. Chem. 2002, 12, 1765-1770.

(169) Jana, N. R.; Gearheart, L.; Murphy, C. J. Adv.Mater. 2001, 13, 13891393.

(170) Wei, Z.; Zamborini, F. P. Langmuir 2004, 20, 11301-11304.

(171) Lee, T.; Chen, Y. MRS Bull. 2012, 37, 144-149.

(172) Larentis, S.; Nardi, F.; Balatti, S.; Gilmer, D. C.; Ielmini, D. Electron Devices, IEEE Transactions on 2012, 59, 2468-2475.

(173) Cho, B.; Kim, T.-W.; Choe, M.; Wang, G.; Song, S.; Lee, T. Org. Electron. 2009, 10, 473-477.

(174) McCarley, R. L.; Irene, E. A.; Murray, R. W. J. Phys. Chem. 1991, 95, 2492-2498.

(175) Nie, Z.; Petukhova, A.; Kumacheva, E. Nat. Nanotechnol. 2010, 5, 1525. 
(176) Corr, S. A.; Byrne, S. J.; Tekoriute, R.; Meledandri, C. J.; Brougham, D. F.; Lynch, M.; Kerskens, C.; O'Dwyer, L.; Gun'ko, Y. K. J. Am. Chem. Soc. 2008, 130, 4214-4215.

(177) Gao, H.; Bao, C.; Yu, T.; Yao, Y.; Li, F.; Yuan, Y.; Liu, J.; Zou, Z. RSC Adv 2014, 4, 10519-10524.

(178) Liang, Y.; Liu, P.; Yang, G. Cryst. Growth Des. 2014, 14, 5847-5855.

(179) Cui, Y.; Björk, M. T.; Liddle, J. A.; Sönnichsen, C.; Boussert, B.;

Alivisatos, A. P. Nano Lett. 2004, 4, 1093-1098.

(180) Yin, D.; Horiuchi, S.; Morita, M.; Takahara, A. Langmuir 2005, 21, 9352-9358.

(181) Mirkin, C. A.; Letsinger, R. L.; Mucic, R. C.; Storhoff, J. J. Nature 1996, 382, 607-609.

(182) Wei, G.; Wang, L.; Zhou, H.; Liu, Z.; Song, Y.; Li, Z. Appl. Surf. Sci. 2005, 252, 1189-1196.

(183) Wang, H.; Chen, L.; Shen, X.; Zhu, L.; He, J.; Chen, H. Angew. Chem. Int., Ed. 2012, 51, 8021-8025.

(184) Shiers, M. J.; Leech, R.; Carmalt, C. J.; Parkin, I. P.; Kenyon, A. J. Adv.Mater. 2012, 24, 5227-5235.

(185) Lin, S.; Li, M.; Dujardin, E.; Girard, C.; Mann, S. Adv. Mater. 2005, 17, 2553-2559.

(186) Grzelczak, M.; Vermant, J.; Furst, E. M.; Liz-Marzán, L. M. ACS Nano 2010, 4, 3591-3605.

(187) Brown, D. A.; Jong-Hoon, K.; Hyun-Boo, L.; Fotouhi, G.; Kyong-Hoon, L.; Wing Kam, L.; Jae-Hyun, C. Sensors 2012, 12, 5725-5751.

(188) Wang, J.-C.; Neogi, P.; Forciniti, D. J. Chem. Phys. 2006, 125, 194717.

(189) Yang, Y.; Matsubara, S.; Nogami, M.; Shi, J.; Huang, W. Nanotechnol 2006, 17, 2821.

(190) Yilmaz, C.; Cetin, A. E.; Goutzamanidis, G.; Huang, J.; Somu, S.; Altug, H.; Wei, D.; Busnaina, A. ACS Nano 2014, 8, 4547-4558.

(191) Ward, D. R.; Halas, N. J.; Ciszek, J. W.; Tour, J. M.; Wu, Y.; Nordlander, P.; Natelson, D. Nano Lett. 2008, 8, 919-924.

(192) Yoon, H. P.; Maitani, M. M.; Cabarcos, O. M.; Cai, L.; Mayer, T. S.; Allara, D. L. Nano Lett. 2010, 10, 2897-2902.

(193) Waser, R. J. Nanosci. Nanotechnol. 2012, 12, 7628-7640.

(194) Waser, R.; Valov, I. ECS Trans. 2009, 25, 431-437. 
(195) Liu, Q.; Sun, J.; Lv, H.; Long, S.; Yin, K.; Wan, N.; Li, Y.; Sun, L.; Liu, M. Adv.Mater. 2012, 24, 1844-1849.

(196) Lai, S. K. IBM J. Res. Dev. 2008, 52, 529-535.

(197) Wang, L.; Gai, S. Contemp. Phys. 2014, 55, 75-93.

(198) Ielmini, D. Microelectronic Engineering 2009, 86, 1870-1875.

(199) Lu, C.-Y.; Hsieh, K.-Y.; Liu, R. Microelectron.Eng. 2009, 86, 283-286.

(200) Wang, L.; Yang, C.; Wen, J.; Gai, S. J. Nanomater. 2014, 2014, 10.

(201) Kim, K.; Lee, S. Y. Microelectron.Eng. 2007, 84, 1976-1981.

(202) Cong, X.; Xiangyu, D.; Jouppi, N. P.; Yuan, X. In Design, Automation \& Test in Europe Conference \& Exhibition (DATE), 2011 2011, p 1-6.

(203) Liu, Q.; Long, S.; Wei, W.; Tanachutiwat, S.; Yingtao, L.; Qin, W.; Manhong, Z.; Zongliang, H.; Junning, C.; Liu, M. Electron. Devic. Lett. 2010, 31, 1299-1301.

(204) Shima, H.; Takano, F.; Muramatsu, H.; Akinaga, H.; Tamai, Y.; Inque, I. H.; Takagi, H. Appl. Phys. Lett. 2008, 93, 113504.

(205) Myungwoo, S.; Joonmyoung, L.; Jubong, P.; Jungho, S.; Godeuni, C.; Seungjae, J.; Wootae, L.; Seonghyun, K.; Sangsu, P.; Hyunsang, H. Electron. Devic. Lett. 2011, 32, 1579-1581.

(206) Waser, R.; Dittmann, R.; Staikov, G.; Szot, K. Adv. Mater. 2009, 21, 2632-2663.

(207) Peng, S.; Zhuge, F.; Chen, X.; Zhu, X.; Hu, B.; Pan, L.; Chen, B.; Li, R.W. Appl. Phys. Lett. 2012, 100, 072101-072104.

(208) Jang, H.-J.; Kirillov, O. A.; Jurchescu, O. D.; Richter, C. A. Appl. Phys. Lett. 2012, 100, 043510-043514.

(209) Hsiung, C.-P.; Liao, H.-W.; Gan, J.-Y.; Wu, T.-B.; Hwang, J.-C.; Chen, F.; Tsai, M.-J. ACS Nano 2010, 4, 5414-5420.

(210) Jiang, X. L.; Zhao, Y. G.; Chen, Y. S.; Li, D.; Luo, Y. X.; Zhao, D. Y.; Sun, Z.; Sun, J. R.; Zhao, H. W. Appl. Phys. Lett. 2013, 102, 253507/253501$253507 / 253505$.

(211) Son, D. I.; Park, D. H.; Kim, J. B.; Choi, J.-W.; Kim, T. W.; Angadi, B.; Yi, Y.; Choi, W. K. J. Phys. Chem. C 2010, 115, 2341-2348.

(212) Chen, G.; Song, C.; Chen, C.; Gao, S.; Zeng, F.; Pan, F. Adv. Mater. 2012, 24, 3515-3520.

(213) Nian, Y. B.; Strozier, J.; Wu, N. J.; Chen, X.; Ignatiev, A. Phys. Rev. Lett. 2007, 98, 146403. 
(214) Guo, X.; Schindler, C.; Menzel, S.; Waser, R. Appl. Phys. Lett. 2007, $91,133513 / 133511-133513 / 133513$.

(215) Schindler, C.; Thermadam, S. C. P.; Waser, R.; Kozicki, M. N. IEEE. T. Electron. Dev. 2007, 54, 2762-2768.

(216) Waser, R.; Aono, M. Nat. Mater. 2007, 6, 833-840.

(217) Kozicki, M. N.; Mira, P.; Mitkova, M. Nanotechnol 2005, 4, 331-338.

(218) Tsunoda, K.; Fukuzumi, Y.; Jameson, J. R.; Wang, Z.; Griffin, P. B.; Nishi, Y. Appl. Phys. Lett. 2007, 90, 113501/113501-113501/113503.

(219) Yang, L.; Kuegeler, C.; Szot, K.; Ruediger, A.; Waser, R. Appl. Phys. Lett. 2009, 95, 013109/013101-013109/013103.

(220) Jafar, M.; Haneman, D. Phys. Rev. B: Condens. Matter 1994, 49, 13611-13615.

(221) Jo, S. H.; Kim, K.-H.; Lu, W. Nano Lett. 2009, 9, 870-874.

(222) Yang, J. J.; Pickett, M. D.; Li, X.; Ohlberg, D. A. A.; Stewart, D. R.; Williams, R. S. Nat. Nanotechnol. 2008, 3, 429-433.

(223) Yang, J. J.; Miao, F.; Pickett, M. D.; Ohlberg, D. A. A.; Stewart, D. R.; Lau, C. N.; Williams, R. S. Nanotechnol 2009, 20, 215201/215201-215201/215209.

(224) Jo, S. H.; Kim, K. H.; Lu, W. Nano Lett 2009, 9, 496-500.

(225) Wang, Z.; Zeng, F.; Yang, J.; Chen, C.; Pan, F. ACS Appl. Mater. Interfaces 2012, 4, 447-453.

(226) Choi, B. J.; Jeong, D. S.; Kim, S. K.; Rohde, C.; Choi, S.; Oh, J. H.; Kim, H. J.; Hwang, C. S.; Szot, K.; Waser, R.; Reichenberg, B.; Tiedke, S. J. Appl. Phys. 2005, 98, 033715/033711-033715/033710.

(227) Kim, D. C.; Seo, S.; Ahn, S. E.; Suh, D. S.; Lee, M. J.; Park, B. H.; Yoo, I. K.; Baek, I. G.; Kim, H. J.; Yim, E. K.; Lee, J. E.; Park, S. O.; Kim, H. S.; Chung, U. I.; Moon, J. T.; Ryu, B. I. Appl. Phys. Lett. 2006, 88, 202102/202101-202102/202103.

(228) Lee, D.; Seong, D.-j.; Jo, I.; Xiang, F.; Dong, R.; Oh, S.; Hwang, H. Appl. Phys. Lett. 2007, 90, 122104/122101-122104/122103. 47-50.

(229) Terabe, K.; Hasegawa, T.; Nakayama, T.; Aono, M. Nature 2005, 433,

(230) Strukov, D. B.; Snider, G. S.; Stewart, D. R.; Williams, R. S. Nature 2008, 453, 80-83.

(231) Jo, S. H.; Kim, K.-H.; Lu, W. Nano Lett. 2009, 9, 496-500.

(232) Dong, Y.; Yu, G.; McAlpine, M. C.; Lu, W.; Lieber, C. M. Nano Lett. 2008, 8, 386-391. 
(233) Moon, T.; Kang, J.; Han, Y.; Kim, C.; Jeon, Y.; Kim, H.; Kim, S. ACS Appl. Mater. Interfaces 2011, 3, 3957-3961.

(234) Liao, Z.-M.; Hou, C.; Zhang, H.-Z.; Wang, D.-S.; Yu, D.-P. Appl. Phys. Lett. 2010, 96, 203109/203101-203109/203103.

(235) Rosezin, R.; Meier, M.; Breuer, U.; Ku; x; geler, C.; Waser, R. IEEE Trans. Nanotechnol. 2011, 10, 338-343.

(236) Meier, M.; Gilles, S.; Rosezin, R.; Schindler, C.; Trellenkamp, S.; Ruediger, A.; Mayer, D.; Kuegeler, C.; Waser, R. Microelectron. Eng. 2009, 86, 1060-1062.

(237) Pi, C.; Ren, Y.; Chim, W. K. Nanotechnol 2010, 21, 085709/085701$085709 / 085708$.

(238) Jin, Z.; Liu, G.; Wang, J. J. Mater. Chem. C 2013, 1, 3282-3286.

(239) Liang, C.; Terabe, K.; Hasegawa, T.; Aono, M. Nanotechnol 2007, 18, 485202/485201-485202/485205.

(240) Dasari, R.; Zamborini, F. P. Anal. Chem. 2015.

(241) Liu, Y.; Qiao, L.; Liu, L.; Guo, R. Colloids Surf., A 2015, 474, 92-100.

(242) Li, W.; Camargo, P. H. C.; Lu, X.; Xia, Y. Nano Lett. 2009, 9, 485-490.

(243) Olson, J.; Dominguez-Medina, S.; Hoggard, A.; Wang, L.-Y.; Chang, W.-S.; Link, S. Chem.Soc.Rev. 2015, 44, 40-57.

(244) Kelly, K. L.; Coronado, E.; Zhao, L. L.; Schatz, G. C. J. Phys. Chem. B. 2003, 107, 668-677.

(245) Hartland, G. V. Chem.Rev. 2011, 111, 3858-3887.

(246) Gramotnev, D. K.; Bozhevolnyi, S. I. Nat.Photon. 2010, 4, 83-91.

(247) Barnes, W. L.; Dereux, A.; Ebbesen, T. W. Nature 2003, 424, 824-

830.

(248) Kim, K.; Shin, D.; Kim, K. L.; Shin, K. S. Phys.Chem.Chem.Phys. 2012, 14, 4095-4100.

(249) Huang, Y.-F.; Wu, D.-Y.; Zhu, H.-P.; Zhao, L.-B.; Liu, G.-K.; Ren, B.; Tian, Z.-Q. Phys.Chem.Chem.Phys. 2012, 14, 8485-8497.

(250) Choi, H.-K.; Shon, H. K.; Yu, H.; Lee, T. G.; Kim, Z. H. J. Phys. Chem. Lett. 2013, 4, 1079-1086.

(251) Chen, G.; Wang, Y.; Yang, M.; Xu, J.; Goh, S. J.; Pan, M.; Chen, H. J. Am.Chem.Soc. 2010, 132, 3644-3645. 


\section{APPENDIX}

\section{Chapter V was reproduced with permission from the American Chemical}

\section{Society, Copyright 2015.}

ACSPublications Title:

Most Trusted. Most Cited. Most Read.
Surfactant-Assisted Voltage-

Driven Silver Nanoparticle Chain

Formation across Microelectrode

Gaps in Air

Author: Nidhi Shah, Francis P. Zamborini

Publication: ACS Nano

Publisher: American Chemical Society

Date: Oct 1, 2015

Copyright (C) 2015, American Chemical Society

\section{PERMISSION/LICENSE IS GRANTED FOR YOUR ORDER AT NO CHARGE}

This type of permission/license, instead of the standard Terms \& Conditions, is sent to you because no fee is being charged for your order. Please note the following:

- Permission is granted for your request in both print and electronic formats, and translations.

- If figures and/or tables were requested, they may be adapted or used in part.

- Please print this page for your records and send a copy of it to your publisher/graduate school.

- Appropriate credit for the requested material should be given as follows: "Reprinted (adapted) with permission from (COMPLETE REFERENCE CITATION). Copyright (YEAR) American Chemical Society." Insert appropriate information in place of the capitalized words.

- One-time permission is granted only for the use specified in your request. No additional uses are granted (such as derivative works or other editions). For any other uses, please submit a new request. 
CURRICULUM VITAE

\author{
Nidhi Shah \\ Department of Chemistry \\ University of Louisville, \\ Louisville, KY 40292 \\ Phone: 502-345-6383 \\ Email: nidhi1209@gmail.com
}

\title{
EDUCATION BACKGROUND
}

University of Louisville (Louisville, KY)

Ph.D. in Chemistry

Dec 2015

Advisor: Dr Francis P. Zamborini

Dissertation title: Voltage-induced Formation of One-Dimensional Silver Nanoparticle Chains Connecting Microgap Electrodes: Mechanism, Resistive Switching, and Analytical Applications

Maharaja Sayajirao University of Baroda (Gujarat, India)

M.S. in Chemistry

June 2006

Thesis title: Synthetic Studies of $\mathrm{C}_{3}$ Symmetric Chiral Molecules

Maharaja Sayajirao University of Baroda (Gujarat, India)

B.S. in Chemistry

June 2004

ACADEMIC RESEARCH EXPERIENCE

University Of Louisville (Louisville, KY)

Ph.D. Researcher

2010-2015 


\section{Research Summary:}

We electrochemically deposited Ag onto 5-micron gap Au interdigitated array (IDA) electrodes as in our previous work, but in the presence of CTAB. Subsequent application of 5-10 V to the IDA in air led to the formation of 1D Ag NP chains connecting the electrode gaps. This work importantly provides a new method for forming 1D of Ag NP chains across micron-sized electrode gaps in air. We used this approach to fabricate interesting plasmonic devices, SERS substrates, and resistive switches. This approach is useful for gaining a better understanding of the electrochemical formation of 1D metal nanochain and filament structures in electrochemical systems. We also electrodeposited different metals like $\mathrm{Cu}, \mathrm{Pd}$ and $\mathrm{Au}$ and studied the chain formation process and switching studies.

\section{Research 1:}

$>$ Studied the formation of silver nanoparticle (Ag NP) chains formed at microelectrode gaps upon application of voltage using Interdigitated Array (IDA) devices.

$>$ Carried out electrodeposition of silver on IDA devices and observed formation of Ag NP chains upon application of voltage in air.

$>$ Characterized the devices using scanning electron microscopy, electronic measurements, and light optical scattering.

\section{Research 2:}

$>$ Studied the resistive switching behavior of Ag NP chains.

$>$ Tested endurance and switching speed of Ag NP chains.

$>$ Characterized the devices using scanning electron microscopy and electronic measurements.

\section{Research 3:}

Studied the light scattering properties of Ag NP chains.

$>$ Performed surface-enhanced Raman studies with Ag NP chains.

$>$ Measured the dark field light scattering spectrum of Ag NP chains.

\section{TEACHING EXPERIENCE}

\section{University of Louisville (Louisville, KY)}

Teaching Assistant

Conducted laboratory sessions, student consultations and performance evaluations.

Led weekly laboratory and/or problem-solving and discussion sessions for students. 
Assisted in Teaching for 4 different laboratory courses (analytical and organic), grading and assessment.

CHEM 343: Organic Laboratory I: Techniques and Intro to Synthesis and Characterization

Fall 2014

Fundamental aspects of organic molecular structure

$>$ Stereochemistry of compounds

$>$ Introduction of basic themes in organic reactivity

$>$ Spectroscopic determination of compounds with emphasis on NMR, GC and IR

$>$ Principles of chromatography, partition coefficient, stationary phases, mobile phases, band spreading, plate height, Van-Deemter equation

\section{CHEM 207 and CHEM 208: Introduction to Chemical} Analysis

$>$ Titration methods

$>$ Use of indicators

$>$ Gravimetric analysis

$>$ Spectrophotometry: measuring transmittance, absorbance, and Beers law

$>$ Acid-Base titration

$>$ Intro to statistics: significant figures, types of errors, accuracy, precision, t-test, Qtest

$>$ Atomic emission spectroscopy

$>$ Chelation and EDTA titrations, use of indicators

$>$ Redox reactions/titrations

\section{CHEM 344: Organic Laboratory II: Synthesis and Characterization Fall 2010}

$>$ Basic synthesis, purification and characterization techniques of organic chemistry

$>$ Critical interpretation of experimental data

\section{PUBLICATIONS}

Nidhi Shah and Francis P. Zamborini. "Surfactant-Assisted Voltage-Driven Silver Nanoparticle Chains across Microelectrode Gaps in Air", ACS Nano, 2015, 9, 10278 10286

Nidhi Shah and Francis P. Zamborini. "Resistive Switching of Electrochemically Synthesized One-Dimensional Silver Nanoparticle Chains”, Langmuir Letter (in preparation) 


\section{PRESENTATIONS}

Nidhi Shah and Francis P. Zamborini. "Voltage Driven Silver Nanoparticle Chains across Electrode Gaps for Conductance Switching”. Gordon Conference, Springfield, MA. June 15-20, 2014- Poster presentation

Nidhi Shah and Francis P. Zamborini. "Electrochemical Deposition of Silver Nanowires/Nanorods by Seed- Mediated Growth." Central Regional Meeting of the American Chemical Society, Detroit, MI. June 08-10, 2012-Oral Presentation

Nidhi Shah and Francis P. Zamborini. "Electrochemical Deposition of Silver Nanorods and Nanowires Directly on Indium tin oxide and Micro gap Electrodes.” Central Regional Meeting of the American Chemical Society, Indianapolis, IN June 08-10, 2011 Poster Presentation

\section{ADVANCE INSTRUMENTATION SKILLS}

Electrochemical Techniques: Cyclic Voltammetry, Chronocoulometry, and Chronoamperometry

Microscopic Techniques: Scanning Electron Microscopy (SEM), Light and Dark Field Optical Microscopy

Spectroscopy Techniques: UV-Vis, Raman and Atomic Absorption Spectroscopy

\section{AWARDS}

Doctoral Dissertation Completion Award

Graduate tuition award
Summer 2015

Spring 2015

\section{MEMBERSHIPS}

American Chemical Society Member

International Student Association, University of Louisville 


\section{REFERENCES}

Dr Francis P. Zamborini (Ph.D. Advisor and Professor)

Director of Graduate Studies

Department of Chemistry

University of Louisville

502-852-6550

f.zamborini@louisville.edu

Dr Danielle Franco (Assistant Professor)

Department of Chemistry

University of Louisville

502-852-7229

dbfran01@,louisville.edu

Dr Muriel C. Maurer (Professor)

Department of Chemistry

University of Louisville

502-852-7008

muriel.maurer@louisville.edu 